\title{
Effects of Turning Radius on Skid-Steered Wheeled Robot Power Consumption on Loose Soil
}

\author{
Jean-Sebastien Fiset
}

\author{
A Thesis \\ in \\ The Department \\ of \\ Electrical and Computer Engineering
}

\author{
Presented in Partial Fulfillment of the Requirements \\ for the Degree of Master of Applied Science (Electrical and \\ Computer Engineering) at \\ Concordia University \\ Montreal, Quebec, Canada
}

August 2019

(C) Jean-Sebastien Fiset 2019 


\section{CONCORDIA UNIVERSITY}

\section{School of Graduate Studies}

This is to certify that the thesis prepared

By: $\quad$ Jean-Sebastien Fiset

Entitled Effects of Turning Radius on Skid-Steered Wheeled Robot Power Consumption on Loose Soil

and submitted in partial fulfillment of the requirements for the degree of

\section{Master of Applied Science (Electrical and Computer Engineering)}

complies with the regulations of the University and meets the accepted standards with respect to originality and quality.

Signed by the final Examining Committee:

Examiner, Chair

Dr. Rastko Selmic

Examiner

Dr. Attila Michael Zsaki (BCEE)

Supervisor

Dr. Krzysztof Skonieczny

Approved by

Dr. W. E. Lynch, Chair

Department or Graduate Program Director

, 2019

Dr. Amir Asif, Dean

Gina Cody School of Engineering and

Computer Science 


\begin{abstract}
Effects of Turning Radius on Skid-Steered Wheeled Robot Power

Consumption on Loose Soil
\end{abstract}

Jean-Sebastien Fiset

This research highlights the need for a new power model for skid-steered wheeled robots driving on loose soil and lays the groundwork to develop such a model. State-ofthe-art power modeling assumes hard ground; under typical assumptions this predicts constant power consumption over a range of small turning radii where the inner wheels are rotating backwards. However, experimental results performed both in the field and in a controlled laboratory sandbox show that, on sand, power is not in fact constant with respect to turning radius. Power peaks by $20 \%$ in a newly identified range of turns where the inner wheels rotate backwards but are being dragged forward. This range of turning radii spans from half the rover width to $R^{\prime}$, the radius at which the inner wheel is not commanded to turn. Data shows higher motor torque and wheel sinkage in this range. To progress toward predicting the required power for a skid-steered wheeled robot to maneuver on loose soil, a preliminary version of a two-dimensional slip-sinkage model is proposed, along with a model of the force required to bulldoze the pile of sand that accumulates next to the wheels as it they are skidding. However, this is shown to be a less important factor contributing to the increased power in small-radius turns than the added inner wheel torque induced by dragging these wheels through the piles of sand they excavate by counter-rotation (in the identified range of turns). Finally, since a direct application of a power model is to design energy-efficient paths, time dependency of power consumption is also examined. Experiments show reduced rover angular velocity in sand around turning radii where the inner wheels are not rotated and this leads to the introduction to a new parameter to consider in path planning: angular slip. 


\section{Acknowledgements}

First and foremost, I would like to express my sincere gratitude to my supervisor, Dr. Krzysztof Skonieczny, for his invaluable guidance and support throughout my masters studies. I was privileged to work with a supervisor that cared so much about my research, trusted me with new ideas and shared his vast knowledge throughout our numerous meetings.

Moreover, I am grateful for the help and suggestions of our technical collaborators from Mission Control Space Services Inc.

I would also like thank my labmates and friends that helped me during the last two years. Amongst them, I wish to personally thank Amir Nassiraei for his unconditional and generous assistance on the technical work but also on the writing of this thesis, Tyson Boer for his help in the design of our power measurement unit, and obviously Meysam Effati, with whom I collaborate with from the start of this project. Special thanks to my friends, Vincent Prevost, Gabriel Broday, Luccas Kunzler, Karin Feistel, Shawn McGrory, Parna Niksirat, Amin Haeri, and Adriana Daca, who created a positive and healthy work environment.

Last but not least, I would like to express my profound gratitude to my parents and my girlfriend for their continuous support and encouragements. This accomplishment would not have been possible without them. 


\section{Contents}

List of Figures

List of Tables $\quad$ xiii

List of Symbols $\quad$ xiv

List of Abbreviations $\quad$ xix

1 Introduction $\quad 1$

1.1 Literature Review . . . . . . . . . . . . . . . . . . . 2

1.1.1 Kinematic Model . . . . . . . . . . . . . . . . . . 2

1.1.2 Dynamic Model ..................... 6

1.1 .3 Power Model . . . . . . . . . . . . . . . . . . . 7

1.1.4 Wheel-Soil Interaction . . . . . . . . . . . . . . . 11

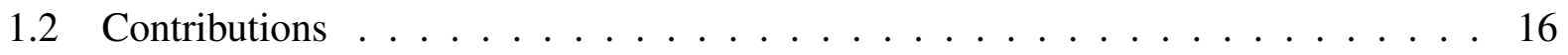

1.3 Thesis Outline . . . . . . . . . . . . . . . . . 17

2 Power Modeling of Skid-Steered Wheeled Robots 19

2.1 Simplifying the Kinematic model . . . . . . . . . . . . . . . . . . . . . 19

2.1.1 Specifying the Velocity Constraint . . . . . . . . . . . . . . . . . 20

2.1.2 Special Case $\left(B / 2<R<R^{\prime}\right) \ldots \ldots \ldots \ldots \ldots$

2.2 Simplifying the ICR-Based Power Model . . . . . . . . . . . . . . . 23

2.3 Summary of Assumptions and Constraints . . . . . . . . . . . . . 25

3 Test Equipment and Setup $\quad 26$

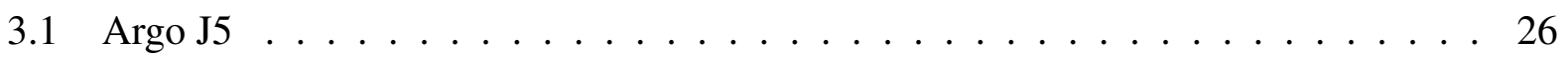

3.2 Clearpath Husky A200 . . . . . . . . . . . . . . . . . . . . . 28

3.3 Five-Axis Single-Wheel Test Bed . . . . . . . . . . . . . . . 32

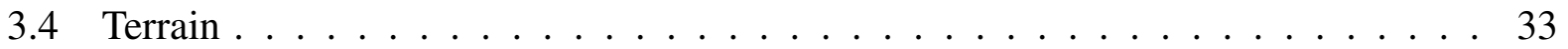


4 Power Model Validation and Limitations $\quad 38$

4.1 Robot's Pitch and Roll Impact on Power Consumption ～. . . . . . . . . . . . . . 38

4.2 Validation of the Power Model on Slopes . . . . . . . . . . . . . . . . . 42

4.3 Power Model Validation on Hard Terrain . . . . . . . . . . . . . . . . . . . . 44

4.4 Limitation of the Existing Model . . . . . . . . . . . . . . . . . . . . 46

4.4.1 Power Consumption over Time . . . . . . . . . . . . . . . . 46

4.4 .2 Average Power Consumption during a Turn . . . . . . . . . . . . . . 48

5 Investigating the Contributing Factors to the Increasing Power Consumption on Sand 51

5.1 Data Post-processing . . . . . . . . . . . . . . . . . . 52

5.2 Torque Comparison . . . . . . . . . . . . . . . . . . . 58

5.3 Sinkage .............................. 60

5.3.1 2D Slip-Sinkage Model . . . . . . . . . . . . . . 61

5.3.2 Comparing the predicted and measured sinkage . . . . . . . . . . . 68

5.4 Sand Bulldozing Force . . . . . . . . . . . . . . . . . . . 72

6 Application of the Improved Power Model $\quad 80$

6.1 CLC vs PLP Paths Comparison . . . . . . . . . . . . . . . . . . . . 81

6.2 Angular slip . . . . . . . . . . . . . . . . . . 86

7 Conclusion and Future Work $\quad 91$

$\begin{array}{ll}\text { Bibliography } & 94\end{array}$

A Derivation of equations for $v_{y}, \omega_{z}, V_{l}$ and $V_{r}$ based on $v_{c}$ and $R$ for left turns $\left(V_{r} \geq V_{l}\right) \quad 98$

B Other Single-Wheel Test Bed Results $\quad 100$

$\begin{array}{ll}\text { C Other CLC vs. PLP Results } & 102\end{array}$

$\begin{array}{lll}\text { D Evaluating the Slip Track } & 105\end{array}$

$\begin{array}{lll}\text { E Unanswered Research Questions } & 108\end{array}$

$\begin{array}{lll}\text { F Submitted Journal Paper } & 110\end{array}$ 


\section{List of Figures}

1.1 Clearpath Husky A200 on the dunes of the White Sands National Monument (WSNM) in New Mexico, USA. . . . . . . . . . . . . . . . . . . . . 1

1.2 Difference between the terms terrain, soil and sand . . . . . . . . . . . . 2

1.3 Schematic figure of a 4-wheel skid-steered wheeled robot performing a left turn. $\vec{v}$ has components $v_{x}$ and $v_{y} \ldots \ldots \ldots \ldots \ldots \ldots \ldots \ldots \ldots \ldots \ldots$

1.4 Heading Angle $\psi_{H}$ used in ICR estimation . . . . . . . . . . . . 5

1.5 A skid-steered wheeled robot climbing a slope of pitch $\theta_{s}$ with a heading angle $\gamma$. . 6

1.6 Circuit diagram of the left and right side of a skid-steered rover [11] . . . . . . . 8

1.7 Schematic figure of a wheel with radius $r$ rotating at angular velocity $\omega$ and moving

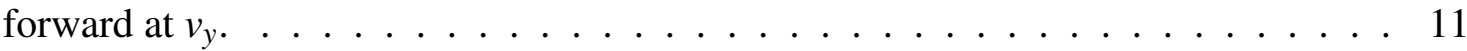

1.8 Estimation model of the bulldozing resistance by Bekker et al. [26] used by Ishigami et al. [14] to compute the sand resistance next a skid-steer rover's wheel . 14

1.9 Geometry of the soil cutting problem used by Skonieczny [28]. $\alpha_{2}$ represents the destructive angle, which is denoted $X_{C}$ in this work. . . . . . . . . . . 16

1.10 High level overview of the topics studied in this research $\ldots \ldots \ldots 17$

2.1 Resulting linear velocity $v_{y}$ and angular velocity $\omega_{z}$ (top), and corresponding left and right commanded linear velocities, i.e. $V_{l}$ and $V_{r}$ respectively, as a function of the commanded turning radius, for a left turn, under a $v_{c}=0.3 \mathrm{~m} / \mathrm{s}$ constraint. The constant $v_{c}$ constraint is defined in Eq. 2.6. . . . . . . . . . . . . 22

2.2 Schematic figure of a four-wheel skid-steered rover performing a turn of radius $B / 2<R<R^{\prime}$. For the inner wheels, the projection of tangential velocity (i.e. actual velocity of the wheel $v^{A C T}$ ) onto the axis of the commanded wheel velocity, $v^{C M D}$, is opposite in direction to $v^{C M D}$.

2.3 Predicting power vs. turning radius using Eq. 2.16 on flat terrain. Comparison with the experimental results on hard ground (Fig. 4.6b) sows concurrence and comparison with the experimental results on sandy soil (Fig. 4.10) shows the limitation of the existing model. 
3.1 Argo J5 (mounted with a landmark prism and an IMU) at the Canadian Space Agency Mars analogue terrain. . . . . . . . . . . . . . . . . . . 27

3.2 a) Clearpath Husky A200 platform mounted with a landmark prism (for position tracking), an IMU and an additional box of sand to place the center of mass of the rover at its geometric center. b) Clearpath Husky A200 placed on the Computerscales Accuset II portable scale.

3.3 Current comparison of the built-in system (dotted line) and custom measurement unit designed for this work (solid line). The left and right current consumption during a right turn of $R=1.5 R^{\prime}$ (top) and during a straight line segment (bottom) on hard ground are shown. . . . . . . . . . . . . . . . . . . 30

3.4 a) Block diagram of the PCB designed and the setup to measure the current fed to the motor driver. b) Custom USB power measurement unit (in red) connected to a shunt sensing resistance (in blue) on the high side of the DC motor driver (in

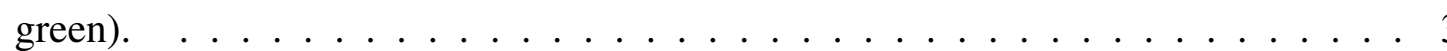

3.5 Custom PCB of the power measurement unit showing the INA226 sensor (U1), the FT232H Single-Channel USB to ${ }^{2} \mathrm{C}$ (U2), the connector to the shunt resistance (J1) and the micro-USB female connector (J2).

3.6 a) A Husky wheel placed in the single-wheel test bed. b) A Husky wheel performing i) a circular arc path of radius $R_{i}$ with a commanded linear velocity $v^{C M D}$, a tangential velocity $v^{A C T}$, and a slip angle $\beta$ and ii) a linear path . . . . . . . 32

3.7 Cone Index (CI) as a function of depth for the fourth run of sandbox turns $\ldots . .34$

3.8 a) GRC-1 cone index gradient as a function of relative density $D_{R}$ of the tests performed by Oravec et al. [29] showing the fitted curve obtained (Eq. 3.2) and the soil condition types. b) Relative density $D_{R}$ obtained by averaging all the cone index gradient $G_{C I}$ from each CI vs. depth line presented in Fig. 3.7 with varying range of depth in the analysis.

3.9 Cone index measurement comparison between the GRC-1 simulant and the sand at the CSA Mars analogue terrain. The scatter points represent the cone index average of all the insertion for each depth and the error bar represent one standard deviation above and under the average. . . . . . . . . . . . . 36

4.1 High level overview of the topics studied in this research, showing the context in which the pitch and roll impact on power consumption is studied. . . . . . . . . . . 39

4.2 Linear regression of the fit against $P=\xi \theta+\eta \phi+\zeta$ for the case of $2 \mathrm{~m}$ turns . . . 40

4.3 Power vs. pitch of the resulting linear equations when $\eta=0$. Slopes of the equations are consistent for $R \geq 1 \mathrm{~m} \ldots \ldots \ldots \ldots$. . . . . . . . 42 
4.4 Fitted power measurements obtained in White Sands National Monument against $P=\frac{c_{1}}{R}+c_{2}+c_{3} \theta$, where $P$ is the power required to perform a turn of radius $R$ with a robot's pitch of $\theta$ degrees. . . . . . . . . . . . . . . . 43

4.5 High level overview of the topics studied in this research, showing the context in which the power model is first validated. . . . . . . . . . . . . . . 44

4.6 a) Power consumption with respect to time as each turn progresses. It can be observed that the power remains relatively constant as small variations are cause by noise in the measurements. b) Average power consumption during a set of $90^{\circ}$ turns, on hard concrete ground. As predicted in Fig. 2.3, the power is constant as $R$ increases from 0 to $2 R^{\prime} / 3$ but the power drops from the $2 R^{\prime} / 3$ to the $R^{\prime}$ turn. . . 45

4.7 High level overview of the topics studied in this research, showing the context in which the limitations of the power model are observed. . . . . . . . . . . . . 46

4.8 Power consumed over time for a set of turns performed in the controlled laboratory sandbox. For $R \leq R^{\prime}$ (dotted lines), power rises as the turn progresses. . . . . . . . 47

4.9 Average power consumed during a set of $90^{\circ}$ turns performed in the laboratory's sandbox, with three $v_{c}$ values. Non-constant power is observed for all cases in the $0 \leq R \leq R^{\prime}$ range (contrast with Fig. 2.3) . . . . . . . . . . . . . . . . 48

4.10 Average power consumed during a set of $90^{\circ}$ turns performed in the laboratory's sandbox (in red) and during the two test campaigns at the CSA Mars analogue terrain (in blue and black), with $v_{c}=0.3 \mathrm{~m} / \mathrm{s} \ldots \ldots \ldots$. . . . . . . . 49

4.11 Average and maximum power consumed during for set of turn tested in the controlled laboratory sandbox (top) and at the CSA Mars analogue terrain (bottom). $\quad 50$

5.1 High level overview of the topics studied in this research, highlighting the conditions in which the contributing factor of increase power consumption are studied. . 52

5.2 Total station data loss correction example for the case of a $R^{\prime}$ turn in the sandbox. The top left plot shows the total station measurements prior to the correction, the bottom left plot shows the $r_{\text {dist }}$ calculation for all the data points (or sample), the top right plot shows the position data with the added points (in pink), and finally the bottom right shows the distances between the point before and after the correction.

5.3 Position of the landmark prism (in red) and the four wheels in the Husky and total station coordinate frames . . . . . . . . . . . . . . . . 54

5.4 Resulting actual inner and outer wheel slip angle, $\beta_{i}$ and $\beta_{o}$ respectively, as a function of the commanded turning radius $R_{C M D}$ of three runs of $90^{\circ}$ turns with the Husky in the GRC-1 simulant of the laboratory sandbox. . . . . . . . . . . . 55 
5.5 For a $R^{\prime}$ turn, this figure is showing the a) position data with the estimated position of $I C R_{v}$ and b) inner and outer wheels' velocity profile before and after moving average filtering. . . . . . . . . . . . . . . . . . 57

5.6 Inner (top) and outer (bottom) wheels torque-proportional curve following Eq. 5.14. For the inner wheels, the $2 R^{\prime} / 3$ case significantly needs more torque than the other radii in order to achieve its desired turn. . . . . . . . . . . . . . 59

5.7 Schematic comparison of a) the usual excavation process by the wheel angular rotation $\left(\omega^{C M D}\right)$ is aligned with the wheel progression $\left(v^{A C T}\right)$, i.e. when $\beta=0^{\circ}$, and (b) the excavation process when the wheel rotation is opposite to the wheel progression, i.e. when $\beta=180^{\circ} \ldots \ldots \ldots$. . . . . . . . . 60

5.8 a) Top view and b) side view of the wheel modeled. . . . . . . . . . . . . . 62

5.9 Husky wheel design showing the important parameters used to model the wheel. . 63

5.10 Measured and predicted sinkage during three linear arc path tests with the singlewheel test bed with $v^{C M D}=0.08 \mathrm{~m} / \mathrm{s}$. The actual velocity was set to $0.02 \mathrm{~m} / \mathrm{s}$ for the $\beta=0^{\circ}$ and $\beta=90^{\circ}$ tests (red and blue line respectively) and to $0 \mathrm{~m} / \mathrm{s}$ for the $100 \%$ slip tests (green line). The latter case was performed to confirm the $k_{E X}$

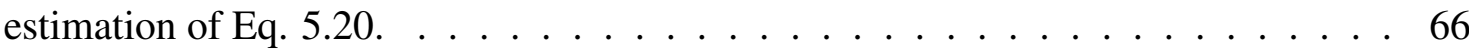

5.11 Estimated inner (top) and outer (bottom) wheel sinkage over time for the set of turning radii tested with the Husky in the sandbox. . . . . . . . . . . . . . 67

5.12 Resulting prediction of the inner (blue) and outer (red) wheel sinkage at the end of the $90^{\circ}$ rotation, with varying turning radius. The overall sinkage, i.e. the sinkage at the geometric center of the vehicle, is also shown and seems to follow the same trend as power vs. $R$ curve seen in Fig. 4.10 . . . . . . . . . . . . . 68

5.13 Measured outer wheel sinkage over time for the set of turning radii tested with the Husky in the sandbox. The outer wheel sinkage was estimated using the variations in the total station measurements of the landmark prism along the $z$ axis, i.e. ${ }^{T S} \Delta z_{l p}$.

5.14 Measured sinkage versus time for the set of turning radii tested (Table 5.2). The wheel sinks more in the $2 R^{\prime} / 3$ case than in the $R^{\prime} / 3$ even if its rotation speed is less than half. The static sinkage of $d_{0}=10 \mathrm{~mm}$ was added to all the measurements, to compare with the sinkage prediction of Fig. 5.11 . . . . . . . . 71

5.15 Side view of a projected wheel moving and bulldozing a sand pile along the $X_{a}$-axis. 73

5.16 Top and front view of a wheel moving along $v^{A C T}$ (or the $X_{a}$-axis) $\ldots \ldots \ldots 74$

5.17 Overall flowchart of the proposed solution . . . . . . . . . . . . . 75

5.18 Inputs of the sand bulldozing force procedure (coming from the data post-processing and 2D slip-sinkage model). . . . . . . . . . . . . . . . . . . 77 
5.19 Resulting sand bulldozing force $F^{S B}$ for the inner (top) and outer (middle) wheels, as well as the sum of the bulldozing force encountered by the four wheels of the Husky rover (bottom) for the set of turns tested. . . . . . . . . . . . . 78

5.20 Average modeled bulldozing force $F^{S B}$ encountered by the rover during a turn with varying turning radius (top) and corresponding added power $\left(P^{A S}\right)$ required to overcome this force (bottom)

6.1 High level overview of the topics studied in this research, showing that the circleline-circle (CLC) and the point turn-line-point turn (PLP) paths were performed both on flat sand and hard concrete terrain. . . . . . . . . . . . . . . . . . 81

6.2 Circle-line-circle (CLC) path and its corresponding point turn-line-poin turn (PLP) path in green and blue, respectively. The start and end angles, $\theta_{\text {start }}$ and $\theta_{\text {end }}$ are shown, as well as the length of the straight line segment, $d_{\text {straight }}$, and the start-toend displacement $d_{p} \ldots \ldots \ldots \ldots \ldots \ldots$

6.3 Energy difference percentage $(\triangle E \%)$ as a function of $R_{A C T} / R_{A C T}^{\prime}$ for a $45^{\circ}-10 \mathrm{~m}$ $-45^{\circ}$ path a) on hard concrete ground and b) in the sand of the CSA Mars analogue terrain

6.4 a) CLC path measured distance as a function of $R_{A C T} / R_{A C T}^{\prime}$ showing the range of $d_{p}$ distance for the $\mathbf{O}_{C L C}$ set when $2<R_{A C T} / R_{A C T}^{\prime}<2.5$. b) PLP path energy consumption as a function of $R_{A C T} / R_{A C T}^{\prime}$ showing the corresponding $\mathbf{O}_{P L P}$ set of tests that are in the same range of $d_{p}$ distance as the $\mathbf{O}_{C L C}$ set. . . . . . . . . . 84

6.5 $\Delta E \%$ over $R_{A C T} / R_{A C T}^{\prime}$ (top) used for the statistical analysis and obtained $p$-value (bottom) showing if the CLC paths with each set of turning radii has a statistically significant decrease (in green) or increase (in red) of energy consumption compared to a PLP path. . . . . . . . . . . . . . . . . . . 85

6.6 High level overview of the topics studied in this research, highlighting that the angular slip is evaluated both on sand and hard ground. . . . . . . . . . . . . 86

6.7 Time to complete a $90^{\circ}$ turn of in the laboratory sandbox (in red) and on hard ground (in blue). The expected time to complete the turn is also plotted in black. . 87

6.8 Actual and commanded angular velocity $\omega_{z}$ as a function of turning radius (top) and the resulting angular slip $s_{\omega}$ (bottom) for hard ground and sand. . . . . . . . . 88

6.9 Angular velocity $\left(\omega_{z}\right)$ over time for the turning radii tested on sand.

6.10 a) Schematic figure showing the orientation of the forces measured by the force/torque sensor (in blue) and how these forces were converted to $F_{\text {tang }}$ and $F_{\text {radial }}$ b) Resulting tangential and radial forces as the wheel progresses on its circular arc path, for the four turns tested. . . . . . . . . . . . . . . . 
6.11 a) Schematic figure of a linear path test with $\beta=90^{\circ}$ showing the orientation of the forces measured. b) Corresponding $F_{z}$ measured of combinations of $3 \mathrm{com}$ manded wheel velocities and 2 actual velocites. The $F_{z}$ force of the braked wheel when $v^{A C T}=0.02 \mathrm{~m} / \mathrm{s}$ and $v^{A C T}=0.04 \mathrm{~m} / \mathrm{s}$ are shown as blue and red solid lines,

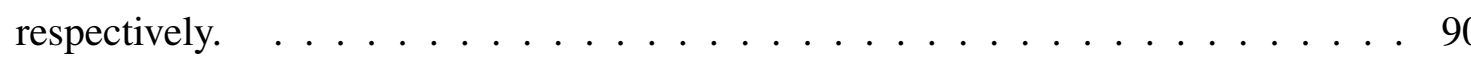

B.1 Relative sinkage for a linear path with $v^{C M D}=0.08 \mathrm{~m} / \mathrm{s}$ and varying slip angle $\beta$ for $v^{A C T}=0.02 \mathrm{~m} / \mathrm{s}$ (top) and $v^{A C T}=0.04 \mathrm{~m} / \mathrm{s}$ (bottom) . . . . . . . . . . 100

B.2 Relative sinkage over time comparison when the wheel is rotating in opposite direction, for three set of slip angle $\beta \ldots \ldots \ldots$. . . . . . . . . . . . . . . .

C.1 Energy difference from the PLP to the CLC path with varying turning radius, depicted as $R_{A C T} / R_{A C T}^{\prime}$ for a $60^{\circ}-8 \mathrm{~m}-30^{\circ}$ path, on hard ground, with constant $v_{c}=0.3 \mathrm{~m} / \mathrm{s}$. The biggest energy reduction occurs at $R_{A C T}=R_{A C T}^{\prime} . \ldots 103$

C.2 Energy difference from the PLP to CLC/CLP/PLC path with varying turning radius, depicted as $R_{A C T} / R_{A C T}^{\prime}$ for a $60^{\circ}-8 \mathrm{~m}-30^{\circ}$ path, on sand, with constant $v_{c}=0.3 \mathrm{~m} / \mathrm{s}$. The biggest energy reduction occurs at around $2 R_{A C T}^{\prime}<R_{A C T}<$ $2.5 R_{A C T}^{\prime} \ldots \ldots \ldots \ldots \ldots \ldots \ldots \ldots \ldots \ldots \ldots \ldots \ldots \ldots \ldots \ldots \ldots$

C.3 Energy difference from the PLP to CLCpath with varying turning radius, depicted as $R_{A C T} / R_{A C T}^{\prime}$ for a $90^{\circ}-10 \mathrm{~m}-90^{\circ}$ path, on sand, with constant $v_{c}=0.3 \mathrm{~m} / \mathrm{s}$. The biggest energy reduction occurs at around $R_{A C T} \approx 2 R_{A C T}^{\prime}$. . . . . . . . 104

C.4 Energy difference from the PLP to CLCpath with varying turning radius, depicted as $R_{A C T} / R_{A C T}^{\prime}$ for a $45^{\circ}-10 \mathrm{~m}-45^{\circ}$ path, on the sand of the CSA Mars analogue terrain, with constant $v_{\max }=0.3 \mathrm{~m} / \mathrm{s}$. The biggest energy reduction occurs at around $2 R_{A C T}^{\prime}<R_{A C T}<2.5 R_{A C T}^{\prime} \ldots \ldots \ldots \ldots$. . . . . . . . . . . . . . . . .

D.1 Comparison between the commanded and actual turning radius, $R_{C M D}$ and $R_{A C T}$, for two runs of turns with the Clearpath Husky, on hard ground. . . . . . . . . . . 105

D.2 $R_{A C T}$ vs. $R_{C M D}$ for all tests . . . . . . . . . . . . 106

D.3 ICR locations obtained for all test cases of the CSA 2017 test campaign . . . . . 107

E.1 Current consumption of the wheel motor of the five-axis single-wheel test bed. . . 109 


\section{List of Tables}

3.1 Geometric parameters of the Argo J5 platform . . . . . . . . . . . . . . 27

3.2 Geometric parameters of the Clearpath Husky A200 platform . . . . . . . . . . 29

3.3 GRC-1 Simulant Parameters Used in this Work . . . . . . . . . . . . . 35

3.4 Comparison of the cone index gradients obtained in the three test campaigns . . . 37

4.1 Fitted Linear Equations . . . . . . . . . . . . . . . . . . . . . 41

$4.295 \%$ Confidence Bounds of the Power Coefficients . . . . . . . . . . . 41

4.3 Power fit results of the $P=c_{1} / R+c_{2}+c_{3} \theta$ for the random drives at the White Sands National Monument . . . . . . . . . . . . . . . . 43

5.1 Husky Wheel Geometry and Model Parameters . . . . . . . . . . . . . . 65

5.2 Parameters to reproduce the Husky's inner wheel trajectories tested . . . . . . . . 70

$6.1 p$-value obtained from the statistical analysis using 5 slices of turning radii $\ldots . .85$

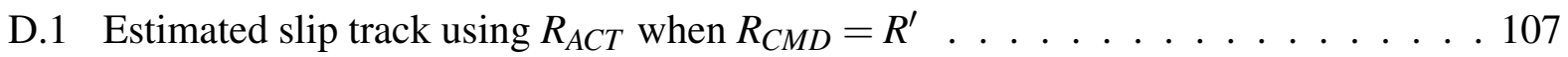




\section{List of Symbols}

$\begin{array}{ll}B & \text { Width of the rover [m] } \\ B_{c} & \text { Wheel contact patch thickness [m] } \\ B_{g} & \text { Wheel grouser width [m] } \\ B_{s} & \text { Slip track [m] } \\ B_{w} & \text { Wheel thickness [m] } \\ c & \text { Soil cohesion [kPa] } \\ C I & \text { Cone Index [kPa] } \\ C_{x}, C_{y} & \text { Distance from the geometric center to the center of mass, } \\ & \text { along the vehicle's } X \text { and } Y \text { axis [m] } \\ C_{d} & \text { Resistive term used in dynamic model } \\ c_{1}, c_{2}, c_{3} & \text { Coefficient used to simplify the existing power model } \\ d & \text { Wheel sinkage [m] } \\ d_{a v g} & \text { Average of front and back of the wheel sinkage [m] } \\ d_{i}, d_{o} & \text { Inner and outer wheel sinkage [m] } \\ d_{i f}, d_{i b}, d_{o f}, d_{o b} & \text { Inner front, inner back, outer front, outer back wheel sink- } \\ d_{I C R} & \text { age [m] } \\ & \text { Distance between a wheel and its instantaneous center of } \\ D_{R} & \text { rotation [m] } \\ d_{s t r a i g h t} & \text { Relative soil density [\%] } \\ d_{0} & \text { Distance of the straight line segment of a CLC or PLP path } \\ e_{c w} & \text { [m] } \\ F_{i}, F_{o} & \text { Static wheel sinkage [m] } \\ F_{R_{i}}, F_{R_{o}} & \text { Ratio of the the width of the wheel contact patch }\left(B_{c}\right) \text { over }\end{array}$


$F_{S}$

$F^{S B}$

$F_{i}^{S B}, F_{o}^{S B}, F_{t}^{S B}$

$g$

$G$

$G_{C I}$

$G_{d}$

$g_{N}, g_{E}, g_{\omega}, g_{r}, g_{l}, g_{x}$

$g_{\text {ratio }}$

$h$

$h_{g c}$

$h_{f}$

$h_{q}$

$h_{q}^{M A X}$

$H_{p}$

$I_{m}$

$I_{\alpha_{1}}, I_{\alpha_{2}}$

$I C R_{v}, I C R_{l}, I C R_{r}$

$j$

K

$k_{c}$

$k_{s}, k_{s}$

$k_{E X}$

$K_{T}$

$k_{\phi}$

$k_{1}, k_{2}$

$l_{g}$

$m$

$M_{d}$
Reaction force to overcome the bulldozing resistance in Ishigami model $[\mathrm{N}]$

Sand bulldozing force $[\mathrm{N}]$

Inner, outer wheel and total sand bulldozing force $[\mathrm{N}]$

Gravitational constant $\left[\mathrm{m} / \mathrm{s}^{2}\right]$

Internal rolling resistance coefficient

Cone index gradient $[\mathrm{kPa} / \mathrm{mm}]$

Gravitation term used in dynamic model

Zero-mean gaussian noises added to each elements of the state vector in the ICR Extended Kalman Filter

Motor gear ratio

Grouser height [m]

Rover ground clearance [m]

Additional height that the shear plane passed through above the soil level [m]

Height of the bulldozed sand pile [m]

Maximum height of the bulldozed sand pile [m]

Experimentally determined constant in Vasil'ev slipsinkage model

DC motor current [A]

Boolean indicator functions used in Skonieczny model

Instataneous center of rotation of the vehicle, the left wheel set, and the right wheel set [m]

Shear displacement [m]

Shear deformation modulus

Pressure sinkage modulus for cohesion stress $\left[\mathrm{N} / \mathrm{m}^{n+1}\right]$

Sinkage parameters of the volume of sand added in front and on the side of the wheel

Wheel sand excavation efficiency

Torque constant [Nm/A]

Pressure sinkage modulus for internal friction angle $\left[\mathrm{N} / \mathrm{m}^{n+2}\right]$

DC motor constants $[\mathrm{Nm} / \sqrt{W}]$

Grouser thickness [m]

Rover mass [kg]

Rover mass matrix used in dynamic model 


\begin{tabular}{|c|c|}
\hline$\dot{N}, \dot{E}$ & Rover linear velocity along the North and East axis $[\mathrm{m} / \mathrm{s}]$ \\
\hline$n_{s}$ & Sinkage exponent used in Bekker model \\
\hline$n_{g}$ & Number of grousers on a wheel \\
\hline$p$ & Normal force/load applied [N] \\
\hline$p_{i}, p_{o}$ & Normal force acting on the inner and outer wheels $[\mathrm{N}]$ \\
\hline$p_{1}, p_{2}, p_{3}, p_{4}$ & $\begin{array}{l}\text { Normal force acting on the front left, front right, back left } \\
\text { and back right wheel }[\mathrm{N}]\end{array}$ \\
\hline$P$ & Electrical power consumption of the rover $[\mathrm{W}]$ \\
\hline$P^{A S}$ & $\begin{array}{l}\text { Additional rover power required to overcome the sand bull- } \\
\text { dozing force [W] }\end{array}$ \\
\hline$P_{i}^{A S}, P_{o}^{A S}, P_{t}^{A S}$ & $\begin{array}{l}\text { Inner, outer wheel and total additional rover power required } \\
\text { to overcome the respective bulldozing force }[\mathrm{W}]\end{array}$ \\
\hline$P_{l}, P_{r}$ & Left and right motor power $[\mathrm{W}]$ \\
\hline$P_{m}$ & DC motor power $[\mathrm{W}]$ \\
\hline$P_{\text {out }}$ & Output mechanical power $[\mathrm{W}]$ \\
\hline$r$ & Wheel radius $[\mathrm{m}]$ \\
\hline$R$ & Turning radius $[\mathrm{m}]$ \\
\hline$R^{\prime}$ & $\begin{array}{l}\text { Turning radius where the inner wheel are not commanded } \\
\text { to rotate }[\mathrm{m}]\end{array}$ \\
\hline$R_{A C T}$ & Actual/Measured turning radius $[\mathrm{m}]$ \\
\hline$R_{b}$ & Sand bulldozing resistance $[\mathrm{N} / \mathrm{m}]$ \\
\hline$R_{c}$ & Turning radius equal to half of the rover's width $(B / 2)[\mathrm{m}]$ \\
\hline$R_{C M D}$ & Commanded turning radius $[\mathrm{m}]$ \\
\hline$R_{i}$ & Inner wheels turning radius $[\mathrm{m}]$ \\
\hline$R_{m}$ & DC motor electrical resistance $[\Omega]$ \\
\hline$r_{\text {dist }}$ & $\begin{array}{l}\text { Distance between two position measurements over the me- } \\
\text { dian distance }[\mathrm{m}]\end{array}$ \\
\hline$R_{l p}$ & Turning radius the landmark prism's circular arc path $[\mathrm{m}]$ \\
\hline$R_{\min }$ & $\begin{array}{l}\text { Minimal turning radius under the } v_{\max } \text { constraint, when the } \\
\text { outer wheels' velocity is equal to } v_{\max }[\mathrm{m}]\end{array}$ \\
\hline$r_{q}$ & $\begin{array}{l}\text { Horizontal distance betwen the front and peak of the sand } \\
\text { pile }[\mathrm{m}]\end{array}$ \\
\hline$r_{\alpha}$ & $\begin{array}{l}\text { Horizontal distance at soil level from the blade to the front } \\
\text { of the failure plane }[\mathrm{m}]\end{array}$ \\
\hline$s_{y}$ & Longitudinal slip ratio \\
\hline$V_{A D D}$ & Predicted volume of sand added beneath the wheel $\left[\mathrm{m}^{3}\right]$ \\
\hline
\end{tabular}




$v^{A C T}$
$v_{i}^{A C T}, v_{o}^{A C T}$
$v_{c}$
$v^{C M D}$
$v_{i}^{C M D}, v_{i}^{C M D}$
$v_{c o n s t}$
$V_{l}, V_{r}$
$v_{\text {max }}$
$V_{R E M}$
$v_{x}, v_{y}$
$w$
$w_{c}$
$w_{g}$
$T S_{x_{l p}},{ }^{T S} y_{l p}{ }^{T S} z_{l p}$
$X_{C}$
$x_{c}, y_{c}$
$x_{w}$
$\alpha_{1}, \alpha_{2}$
$\beta$
$\beta_{i}, \beta_{o}$
$\gamma$
$\delta$
$\Delta x_{l p}$
$\Delta x_{\text {side }}$
$\Delta z_{4}$

${ }_{\text {ront }}$

Actual wheel linear velocity $[\mathrm{m} / \mathrm{s}]$

Inner and outer actual wheel linear velocity $[\mathrm{m} / \mathrm{s}]$

Total absolute linear velocity $[\mathrm{m} / \mathrm{s}]$

Commanded wheel linear velocity $[\mathrm{m} / \mathrm{s}]$

Inner and outer commanded wheel linear velocity $[\mathrm{m} / \mathrm{s}]$

Constant forward velocity constraint value $[\mathrm{m} / \mathrm{s}]$

Left and right wheel linear velocity $[\mathrm{m} / \mathrm{s}]$

Maximum wheel velocity constraint value $[\mathrm{m} / \mathrm{s}]$

Predicted volume of sand remove from under the wheel $\left[\mathrm{m}^{3}\right]$

Components of the rover's velocity $\vec{v}$ along the rover's $X$ and $Y$ axis $[\mathrm{m} / \mathrm{s}]$

Width of the wheel at the soil level, projected on a plane perpendicular to the wheel displacement [m]

Width of the wheel contact patch [m]

Width of the wheel at the soil level [m]

Coordinates of the landmark prism, in the total station frame $[\mathrm{m}]$

Soil destructive angle [rad]

Coordinate of the center of the circular rover path [m]

Position of the wheel along the $X_{a}$-axis [m]

Rake angle and destructive angle, in Skonieczny model [rad]

Slip angle $\left[^{\circ}\right]$

Inner and outer slip angle $\left[{ }^{\circ}\right]$

Rover's angle of attack when climbing a slope $\left[{ }^{\circ}\right]$

Soil external friction angle $\left[{ }^{\circ}\right]$

Time interval between two measurements [s]

Distance between the landmark prism and the geometric center of the rover [m]

Wheel displacement along the vehicle's $X$-axis [m]

Wheel displacement along the vehicle's $Y$-axis [m]

Experimentally determined constant in Ding slip-sinkage model

Fit coefficient of the power equation as a function of pitch and roll, i.e. $P(\theta, \phi)=\xi \theta+\eta \phi+\zeta$ 


\begin{tabular}{|c|c|}
\hline$\eta_{l}, \eta_{r}$ & Left and right DC motor efficiency \\
\hline$\eta_{e}$ & Power efficiency factor of an electromechanical system \\
\hline$\theta, \phi, \psi$ & Rover's pitch, roll and yaw angles $\left[{ }^{\circ}\right]$ \\
\hline$\theta_{f}, \theta_{r}, \theta_{c}$ & Front angle, rear angle and the sum of the two angle [rad] \\
\hline$\theta_{H}$ & Orientation of the rover in the total station frame [rad] \\
\hline$\theta_{s}$ & Slope pitch angle $\left[{ }^{\circ}\right]$ \\
\hline$\theta_{w}$ & Arbitrary angle along the wheel $[\mathrm{rad}]$ \\
\hline$\theta_{w i}, \theta_{w o}$ & $\begin{array}{l}\text { Inner and outer wheel position, used in dynamic model } \\
{[\mathrm{rad}]}\end{array}$ \\
\hline$\lambda$ & Wheel sinkage ratio \\
\hline$\mu$ & Friction coefficient \\
\hline$\mu_{r}$ & Coefficient of rolling resistance \\
\hline$\mu_{s}$ & Coefficient of skidding friction \\
\hline$\rho$ & Soil dry bulk density $\left[\mathrm{g} / \mathrm{cm}^{3}\right]$ \\
\hline$\tau$ & Motor torque $[\mathrm{Nm}]$ \\
\hline$\tau_{i}, \tau_{o}$ & Inner and outer motor torque $[\mathrm{Nm}]$ \\
\hline$\tau_{i, r e s}, \tau_{o, \text { res }}$ & Friction in the inner and outer driving system \\
\hline$\tau_{s s}$ & Shear stress $[\mathrm{Pa}]$ \\
\hline$\Phi$ & Scaling factor used in the ICR-based power model \\
\hline$\phi_{i}$ & Soil internal friction angle $\left[{ }^{\circ}\right]$ \\
\hline$\varphi_{r}$ & Soil repose angle $\left[{ }^{\circ}\right]$ \\
\hline$\psi_{H}$ & Rover's heading angle $\left[^{\circ}\right]$ \\
\hline$\omega$ & Angular velocity of a wheel $[\mathrm{rad} / \mathrm{s}]$ \\
\hline$\omega^{C M D}$ & Commanded wheel angular velocity $[\mathrm{rad} / \mathrm{s}]$ \\
\hline$\omega_{i}, \omega_{o}$ & Angular velocity of the inner and outer wheels $[\mathrm{rad} / \mathrm{s}]$ \\
\hline$\omega_{l}, \omega_{r}$ & Angular velocity of the left and right wheels [rad/s] \\
\hline$\omega_{m}$ & Motor angular velocity $[\mathrm{rad} / \mathrm{s}]$ \\
\hline$\omega_{z}$ & Rover's angular velocity around its $Z$-axis [rad/s] \\
\hline
\end{tabular}




\section{List of Abbreviations}

$\begin{array}{ll}\text { CLC } & \text { Circle - Line - Circle } \\ \text { CSA } & \text { Canadian Space Agency } \\ \mathrm{I}^{2} \mathrm{C} & \text { Inter Integrated Circuit } \\ \text { ICR } & \text { Instantaneou Center of Rotation } \\ \text { IMU } & \text { Inertial Measurement Unit } \\ \text { PLP } & \text { Point turn - Line - Point turn } \\ \text { ROS } & \text { Robotic Operating System } \\ \text { USB } & \text { Universal Serial Bus } \\ \text { WSNM } & \text { White Sands National Monument }\end{array}$




\section{Chapter 1}

\section{Introduction}

Skid-steer rovers are defined as mobile vehicles with wheels or tracks where the right and left sides are both fixed poiting forward, but independently rotate at different velocities in order to maneuver. These maneuvers range from a turn with zero radius of curvature (point turn), when the wheels on both side are rotating at equal speed in opposite direction, to straight line driving, when both sides are rotating at the same velocity, in the same direction. As the wheels are directly coupled to the motors and do not require a steering mechanism, these rovers are very compact and robust, which makes them suitable for rough terrains. Therefore, due to their maneuverability, mechanical simplicity and robustness, skid-steer rovers are widely used for excavation, construction, military and planetary exploration applications. Energy-efficient navigation is an important aspect of any of these applications, especially when autonomously planning paths in power-starved environments. However, the power consumption for skid-steer vehicles can be high, and also highly variable, compared to other steering mechanism (such as explicit or Ackerman steering) due to the torque

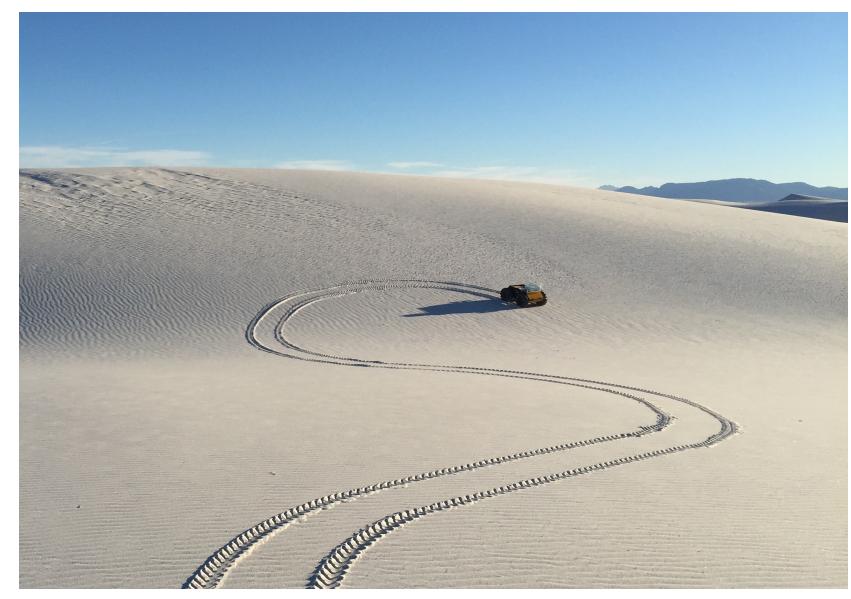

Figure 1.1 Clearpath Husky A200 on the dunes of the White Sands National Monument (WSNM) in New Mexico, USA. 
required to overcome lateral motion resistance while skidding in a turn. Detailed power modeling is thus essential for this important and popular class of mobile robots. It is also important to note that loose soil is frequently encountered in the applications cited above, though skid-steer rover power consumption in loose soil has not yet received much attention in the literature.

Accordingly, the state-of-the-art in power modeling for skid-steer rovers is for motion on rigid terrain. In this research, the applicability of these power models on loose soil is studied, with an explicit consideration of the turning radius (or radius of curvature). This chapter first presents an in-depth review of the literature before establishing the contributions of this research and the outline of the thesis.

\section{Terminology}

Throughout this thesis, an important distinction is made between the terms terrain, soil and sand. As depicted in Fig. 1.2, a terrain includes both its type and its geometry (i.e.slope and roughness for example). Soil is one a type of terrain and includes sand, clay or any other soils.

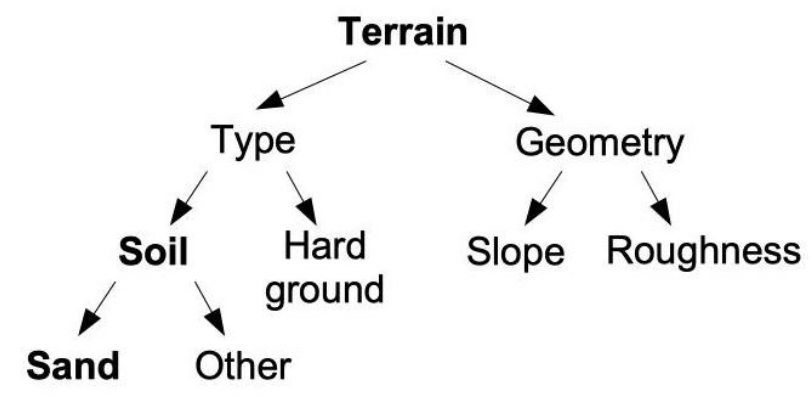

Figure 1.2 Difference between the terms terrain, soil and sand

\subsection{Literature Review}

In order to comprehensively study power modeling for skid-steered wheeled robots, it is necessary to also review their kinematics, dynamics, and wheel-soil interactions.

\subsubsection{Kinematic Model}

For differential wheeled and tracked vehicles, the location of the center of rotation for the vehicle, $I C R_{v}$, is not the same as the center of rotation of each wheel or track set [1], as it can be seen in Fig. 1.3. This figure also shows the vehicle's coordinate frame, which is fixed relative to the vehicle. Due to slippage, the left and right instantaneous centers of rotation (ICR) lie outside of the wheel track and they are expressed with respect to the vehicle's coordinate frame such 
that $I C R_{l}=\left(x_{I C R_{l}}, y_{I C R_{l}}\right)$ and $I C R_{r}=\left(x_{I C R_{r}}, y_{I C R_{r}}\right)$. The vehicle's center of rotation as well as the two ICRs are located on the same line, parallel to the vehicle $X$ axis [1]. The distance from the geometric center of the rover to this parallel line is defined as $y_{I C R_{v}}$ and can be found using Eq. 1.1.

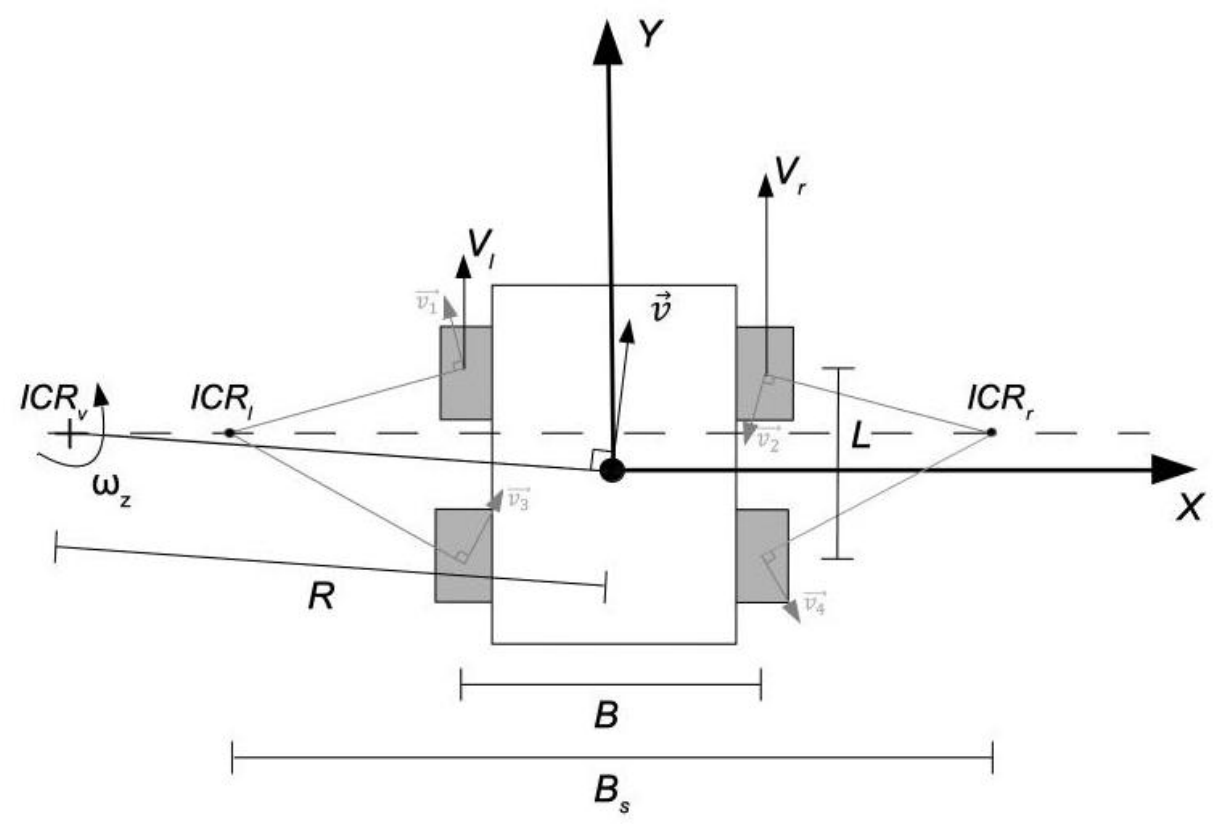

Figure 1.3 Schematic figure of a 4-wheel skid-steered wheeled robot performing a left turn. $\vec{v}$ has components $v_{x}$ and $v_{y}$.

The components along the $X$ axis of the left and right instantaneous centers of rotation can be obtained knowing the linear and angular velocities, as shown in Eq. 1.2 and Eq. 1.3, where $v_{x}$ and $v_{y}$ are the vehicle's linear velocity expressed along the vehicle $X$ and $Y$ axis respectively, $\omega_{z}$ is the angular velocity around the $Z$-axis and $V_{l}$ and $V_{r}$ are the left and right wheel set commanded linear velocities respectively, computed by multiplying the commanded angular velocity of each wheel set by the wheel radius. It is also important to note that the location of the center of mass has an impact on the location of the left and right instantaneous centers of rotation. As it moves closer to one side of the vehicle, the wheels on this side will slip less, thus resulting in an instantaneous center of rotation closer to those wheels [2].

$$
\begin{gathered}
y_{I C R_{v}}=y_{I C R_{l}}=y_{I C R_{r}}=\frac{v_{x}}{\omega_{z}} \\
x_{I C R_{l}}=\frac{V_{l}-v_{y}}{\omega_{z}} \\
x_{I C R_{r}}=\frac{V_{r}-v_{y}}{\omega_{z}}
\end{gathered}
$$


The distance between these two centers of rotation is defined as the slip track $\left(B_{S}\right)$ [3].

$$
B_{s}=x_{I C R_{r}}-x_{I C R_{l}}
$$

As the ICRs always lie outside of the rover's track due to slippage, the slip track is always greater that the track width, i.e. $B_{S} \geq B$ [1][3], where $B$ is the distance between the left wheels center and right wheels center. It is important to note that, even if $x_{I C R_{v}}$ and $R$, i.e. the distance between $I C R_{v}$ and the rover's geometric center, range within $\pm \infty$, the values of $x_{I C R_{l}}, x_{I C R_{r}}$ and $y_{I C R_{v}}$ remain within bounded areas for a specific terrain, regardless of the maneuver [1][3]. Hence, the slip track can be used as a measure of the overall skidding of the wheeled vehicle. A bigger slip track indicates a higher lateral slip for a particular terrain. Accordingly, the ICR positions can help characterize the properties of a terrain [3]. Studies have also shown that knowing the instantaneous center of rotation locations can enhance the pose estimation [1][4] and be used for power modeling [5][6][7].

The following equations are used to express the linear and angular velocity in the body frame when considering slippage.

$$
\begin{gathered}
v_{y}=\frac{V_{l} x_{I C R_{r}}-V_{r} x_{I C R_{l}}}{B_{s}} \\
v_{x}=\frac{\left(V_{r}-V_{l}\right) y_{I C R_{v}}}{B_{s}} \\
\omega_{z}=\frac{V_{r}-V_{l}}{B_{s}}
\end{gathered}
$$

These equations can be rewritten together in a matrix format:

$$
\left[\begin{array}{c}
v_{x} \\
v_{y} \\
\omega_{z}
\end{array}\right]=\frac{1}{B_{s}}\left[\begin{array}{cc}
-y_{I C R_{v}} & y_{I C R_{v}} \\
x_{I C R_{r}} & -x_{I C R_{l}} \\
-1 & 1
\end{array}\right]\left[\begin{array}{c}
V_{l} \\
V_{r}
\end{array}\right]
$$

These kinematics will be further developed in Section 2.1, according to appropriate simplifying assumptions and constraints. 


\section{On-line Estimation the ICR Location}

Pentzer et al. [4] used an Extended Kalman Filter (EKF) is used to estimate the ICR locations. Knowing the kinematic model of a skid-steered vehicle, the velocity along the North and East axis, i.e. $\dot{N}$ and $\dot{E}$, are

$$
\begin{aligned}
& \dot{N}=v_{y} \cos \psi_{H}-v_{x} \sin \psi_{H}, \\
& \dot{E}=v_{y} \sin \psi_{H}-v_{x} \cos \psi_{H} .
\end{aligned}
$$

As shown in Figure 1.4, $\psi_{H}$ is defined an being the angle from the North axis to the $\mathrm{x}$-axis of the vehicle.

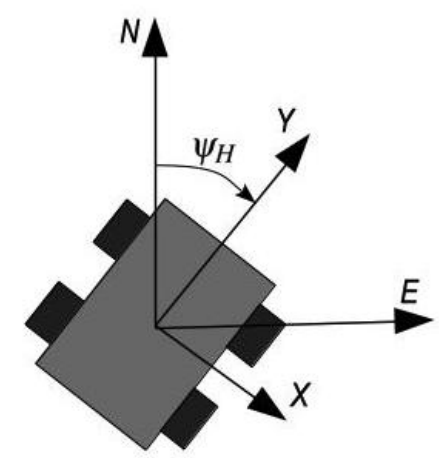

Figure 1.4 Heading Angle $\psi_{H}$ used in ICR estimation

Using Eq. 1.2 and 1.3, an expression for $\omega_{z}$ can be derived and included in the state vector used in the Extended Kalman Filter. Zero-mean gaussian noises $\left(g_{N}, g_{E}, g_{\omega}, g_{r}, g_{l}\right.$ and $\left.g_{x}\right)$ are also added to each element of the state vector. As mentioned, since the instantaneous centers of rotation are bounded to small region regardless of the maneuver, their location can be modeled as constants with an added noise.

$$
\left[\begin{array}{c}
\dot{N} \\
\dot{E} \\
\omega_{z} \\
\dot{y}_{I C R_{r}} \\
\dot{y}_{I C R_{l}} \\
\dot{x}_{I C R_{v}}
\end{array}\right]=\left[\begin{array}{c}
v_{y} \cos \psi_{H}-v_{x} \sin \psi_{H}+g_{N} \\
v_{y} \sin \psi_{H}-v_{x} \cos \psi_{H}+g_{E} \\
\frac{V_{r}-V_{l}}{B_{s}}+g_{\omega} \\
g_{r} \\
g_{l} \\
g_{x}
\end{array}\right]
$$

This state vector is then discretized and used in the algorithm presented in [4] to estimate on-line the position of the instantaneous centers of rotation. 


\subsubsection{Dynamic Model}

The dynamic model proposed in [8], and based on the exponential friction model of [9], helps predict the motor torques for a skid-steered vehicle operating on sloped hard terrain. The model is also valid for all turning radii but limited to small accelerations. In a steady state turn, the dynamic model relates torque and wheel state vectore $q$ such that

$$
M_{d} \ddot{q}+C_{d}(q, \dot{q})+G_{d}(q)=\tau,
$$

where $q=\left[\theta_{w i}, \theta_{w o}\right]^{T}$ is the state vector containing the inner and outer wheel position respectively, $\dot{q}=\left[\omega_{i}, \omega_{o}\right]^{T}$ is angular velocity of the inner and outer wheels, $M_{d}$ is the mass matrix, $C_{d}(q, \dot{q})$ is the resistive term, $G_{d}(q)$ is the gravitational term and finally $\tau=\left[\tau_{i}, \tau_{o}\right]^{T}$ is the torque of the inner and outer wheels' motor. In most of the previous work using this dynamic model, the focus is on steady state maneuvers (acceleration is not considered), causing $M_{d} \ddot{q}=0$. In the case of a steady state left turn (as shown in Figure 1.3), the inner wheels correspond to the the left wheels and the outer wheels are the right wheels.

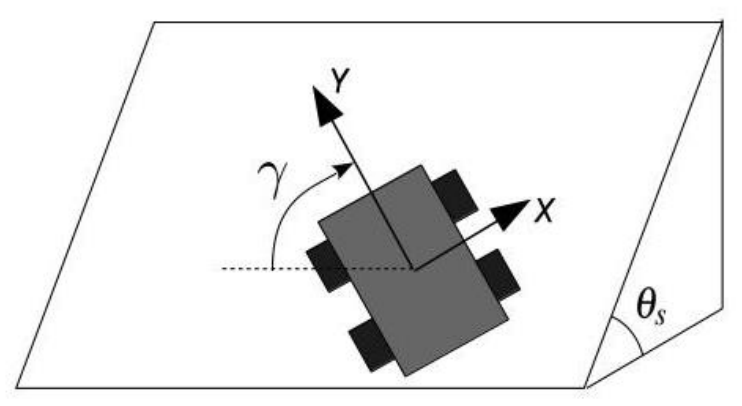

Figure 1.5 A skid-steered wheeled robot climbing a slope of pitch $\theta_{s}$ with a heading angle $\gamma$.

The following analysis for the $C_{d}(q, \dot{q})$ term was initially presented by [9] before being extended for wheeled skid-steered wheeled robots by [8] and then extended by [10] to capture the full range of turning radii and to take into consideration the effect of the slope angle $\theta_{s}$ and the vehicle heading $\gamma$. In these papers, the term takes into account the longitudinal friction forces, the rolling resistance forces and the friction in the driving system for each wheel set. The longitudinal frictional force can be calculated using the relationship between shear stress $\tau_{s s}$ and shear displacement $j$ of Eq. 1.13, where $p$ is the normal pressure, $\mu$ is the coefficient of friction, and $K$ is the shear deformation modulus.

$$
\tau_{s s}=p \mu\left(1-e^{-j / K}\right)
$$


The detailed expression for the inner and outer wheels' frictional force ( $F_{i}$ and $F_{o}$, respectively), shown in [10], considers the shift of the center of mass from the geometric center and assumes a rectangular contact patch for the wheel.

Friction in the driving system is expressed by $\left[\tau_{i, r e s}, \tau_{o, \text { res }}\right]^{T}$ and can be experimentally determined by lifting up the rover to avoid contact between the wheels and the ground and then measuring the motor torque at nominal speed. The rolling resistance forces $\left[F_{R_{i}}, F_{R_{o}}\right]^{T}$ are determined by measuring the motor torque when the rover is moving in straight line at constant speed on flat ground and subtracting the friction in the driving system.

An expression of the normal force for each side of the vehicle based on the vehicle heading and the slope angle is shown in [10]. These normal forces $\left(p_{i}\right.$ and $\left.p_{o}\right)$ are used to estimate coefficient of rolling resistance $\mu_{r}$ for both sets of wheels knowing that $F_{R_{i}}=\mu_{r} p_{i}$ and $F_{R_{i}}=\mu_{r} p_{o}$.

The total resistance term for a turning radius $R$ is expressed as

$$
C_{d}(q, \dot{q})=R\left[\begin{array}{c}
F_{i}+F_{R_{i}} \\
F_{o}+F_{R_{o}}
\end{array}\right]+\left[\begin{array}{c}
\tau_{i, r e s} \\
\tau_{o, r e s}
\end{array}\right]
$$

Finally, in a sloped environment, the gravitational term $G_{d}(q)$ is expressed as

$$
G_{d}(q)=\frac{r m \sin \theta_{s} \sin \gamma}{B}\left[\begin{array}{l}
\frac{B}{2}-C_{x} \\
B \\
\frac{2}{2}+C_{x}
\end{array}\right],
$$

where $m$ is the mass of the vehicle, $B$ is the width of the vehicle and $C_{x}$ is the distance between the center of gravity and geometric center along the vehicle's $X$-axis.

\subsubsection{Power Model}

Three existing power models specific for skid-steer rover operating on hard terrain are explored. The first one relies on the kinematic model and the locations of the rover's instantaneous centers of rotation, the second one is based on the dynamic model presented in the previous section and the last one uses an estimation of the friction coefficient to design a physics-based power model for skid-steered wheeled robots.

\section{Based on Instantaneous Centers of Rotation}

The dynamic friction between the wheels that causes the vehicle to skid during a turn can be modeled using the popular ICR-based power model. Based on the work of [5][6] and adding a 
term with the $\Phi$ coefficient to consider elevation change [7], the power loss of a skid-steered rover can be modeled as

$$
P=\mu\left|\omega_{z}\right| \sum_{n=1}^{N} p_{n}\left\|\vec{a}-\vec{C}_{n}\right\|+G\left(\left|V_{r}\right|+\left|V_{l}\right|\right)+\Phi m g v_{y} \sin \theta,
$$

where $\mu$ is the friction coefficient, $\omega_{z}$ is the angular velocity around the rover's $Z$-axis, $p_{n}$ is the normal force applied on the $n$th wheel, and $\left\|\vec{a}-\vec{C}_{n}\right\|$ is the distance between the $n$th wheel and its respective ICR, $G$ is the internal rolling resistance coefficient, $V_{l}$ and $V_{r}$ are the left and right side velocities, $\Phi$ is a scaling factor, $m$ is the mass of the rover, $g$ is the gravitational constant, $v_{y}$ is the rover's linear velocity along the vehicle's $Y$-axis and $\theta$ is the pitch angle of the rover.

The power model will be further developed according to relevant assumptions and constraints in Section 2.2.

\section{Based on Dynamic Model}

Another approach of power modeling is by separating the left and right mechanical power associated with each side's wheel rotation and also separating the left and right electrical power associated with the electrical resistance on each side. This work, presented by [11] using the exponential friction model of [9], combines two separate power models to generate an estimate of the required power for the entire vehicle. Using the dynamic model of Section 1.1.2, where the torque generated by the left and right motor is expressed as a function of the turning radius $R$, the heading angle $\gamma$ on a slope, and the slope angle $\theta_{s}$, the power for a motor can also be correlated to these three parameters.

As it can be seen in Figure 1.6, the left side wheels are coupled together and mechanically connected to the left motor. The same thing applies for the right side wheels as well.

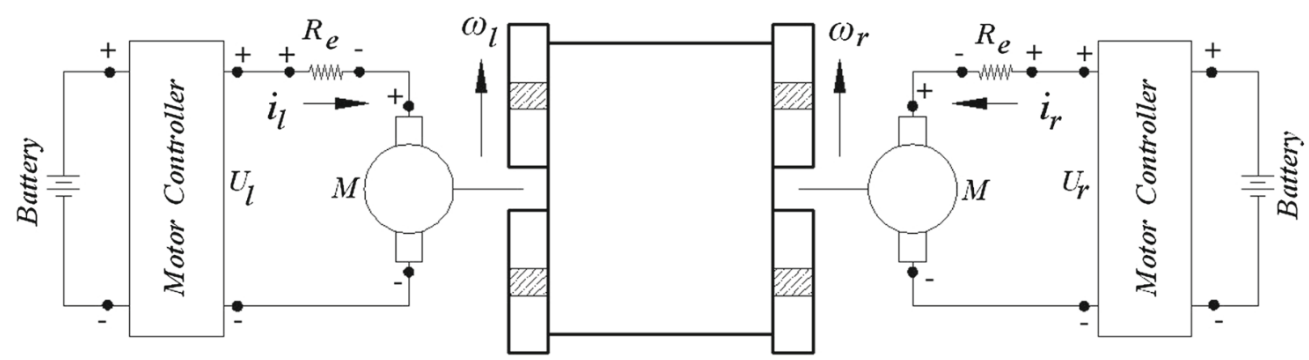

Figure 1.6 Circuit diagram of the left and right side of a skid-steered rover [11]. 
Assuming that the vehicle in Figure 1.6 is performing a counterclockwise turn of radius larger than half the slip track $B_{s}$ (causing $\omega_{l}$ and $\omega_{r}$ to be both positive), the power model of a DC motor is defined as

$$
P_{m}=\omega_{m} \tau+R_{m} I_{m}^{2}
$$

where $\omega_{m}$ is the motor angular velocity, $\tau$ is the outputted torque of the motor, $R_{m}$ is the electrical resistance and $I_{m}$ it the current going through the motor. Knowing this relationship between power and torque, the left and right motor power consumption $\left(P_{l}\right.$ and $\left.P_{r}\right)$ are calculated, assuming an motor efficiency of $\eta_{l}$ and $\eta_{r}$ respectively. Eq. 1.18 and 1.19 define the left and right power as a function of the components of the circuit diagram with an added torque constant $K_{T}$ and gear ratio $g_{\text {ratio }}$.

$$
\begin{aligned}
& P_{l}=\frac{\tau_{l} \omega_{l}}{\eta_{l}}+\left(\frac{\tau_{l}}{K_{T} g_{\text {ratio }} \eta_{l}}\right)^{2} R_{m} \\
& P_{r}=\frac{\tau_{r} \omega_{r}}{\eta_{r}}+\left(\frac{\tau_{r}}{K_{T} g_{\text {ratio }} \eta_{r}}\right)^{2} R_{m}
\end{aligned}
$$

The sum of the left and right power equation gives the total power only when the rotation of the wheels are caused by both motors' applied torque. The power calculated on one side can be negative if the applied torque is 0 and the wheel rotates due to the torque applied by the other side motor. This results in power generation on that side. Since most vehicles do not have the capability of charging the battery, then any negative power calculated should be put to 0 when computing the total power estimate. A further study of this power model is elaborated in [12].

\section{Based on Friction Coefficient}

The work of [13] shows a physics-based approach for power modeling using friction coefficients to estimate the power consumed for a set of parameters such as the location of the center of mass, the terrain type, the vehicle's velocity, the radius of curvature, terrain relief (i.e. geometry) and temperature of the motors. In their research, the motion of a skid-steered vehicle is separated in three cases: when the turning radius $R$ is close to $\infty$ (driving straight), when $R_{c}<R<\infty$ where $R_{c}$ is the distance between the geometric center and a wheel track, i.e. $B / 2$, and finally when $R<R_{c}$. In the second case, they assumed that the outer (or active) wheels are responsible for all the required force to drive the robot along the curved path which is not necessarily the most efficient procedure, especially when dealing with large turning radii. In the third case, when the location of the center of rotation is within the vehicle, the left and right motors generate force to rotate the 
wheels in opposite directions. Both motors are then responsible for overcoming the friction due to skidding.

For a finite turning radius, the friction coefficient is first estimated under the assumption that the rolling and skidding friction act independently on a wheel and they are modeled using the $\mu_{r}$ and $\mu_{s}$ coefficients respectively. Taking into account the rover's geometry, i.e. the $C_{x}$ and $C_{y}$ displacement of the center of mass with respect to the geometric center of the skid-steered vehicle, and the $R$ distance between the geometric center of the vehicle and the center of rotation, the friction coefficient $\mu$ is defined as

$$
\mu=\frac{R+C_{y}}{R+\frac{B}{2}} \sqrt{\frac{\left(\frac{L}{2}-\left|C_{x}\right|\right)^{2}+\left(\frac{B}{2}\right)^{2}}{\left(\frac{L}{2}\right)^{2}+\left(\frac{B}{2}\right)^{2}}} \mu_{r}+\frac{2 L\left(\frac{1}{4}-\left(\frac{C_{x}}{L}\right)^{2}\right)}{R+\frac{B}{2}} \mu_{s} .
$$

This estimated friction coefficient is then used to calculate the total power loss $P_{I N}$ of the vehicle in the following equation:

$$
\begin{aligned}
P_{I N}=\mu^{2} k_{1} m^{2} g^{2} \cos ^{2} \theta_{s} & \pm \mu\left(2 m g k_{1} \sin \theta_{s} \sin \gamma+\omega_{z} k_{2}\right) m g \cos \theta_{s} \\
& +m g \sin \theta_{s} \sin \gamma\left(m g k_{1} \sin \theta_{s} \sin \gamma+\omega_{z} k_{2}\right),
\end{aligned}
$$

where $\mu$ is the friction coefficient, $k_{1}$ and $k_{2}$ are the motor constants, $m$ is the mass of the vehicle, $\theta_{s}$ is the angle of the slope, $\omega$ is the rover's angular velocity, and $\gamma$ is the angle of attack (as seen in Fig. 1.5) of the vehicle. As mentioned, since they take into consideration the increased power consumption as the temperature rises, the motor constants are defined as functions of the motor temperature.

Multiple conclusions are obtained in this paper. As expected, they demonstrated that friction varies with the turning radius and the speed of the robot. They have also shown that the skid friction decreases as the center of mass moves closer to the front or back of the vehicle, and that it increases as the center of mass moves closer to the left and right side of the vehicle. As it can be seen in equation 1.20, longer platforms will also cause the robot to experience more skid. Therefore, the platform geometry should be taken into account when trying to predict the necessary power required, which is not explicitly the case in the ICR-based power modeling, though geometry can affect the ICR locations. 


\subsubsection{Wheel-Soil Interaction}

In this section, previous work related to the interaction of wheels on loose soil is presented. The concept of slip is first introduced as it is a key parameter of the slip-sinkage models discussed afterwards. Then, two relevant references on modeling the sand bulldozing force experienced by a wheel skidding in loose soil are studied and their applicability for our work is examined.

\section{Slip}

When a skid-steer rover is operating on loose terrain, the wheels are often slipping. To evaluate the slip, the difference between the commanded and traveling velocity of the wheel is used in the formulation of the slip ratio in the direction of travel, i.e. $s_{y}$. This longitudinal slip ratio is defined as [14]: abovedisplayshortskip

$$
s_{y}=\frac{r \omega-v_{y}}{r \omega},
$$

where $r$ is the wheel radius, $\omega$ is the commanded angular velocity of the wheel, and $v_{y}$ is the wheel's actual velocity along the direction of commanded travel, as seen in Fig. 1.7.

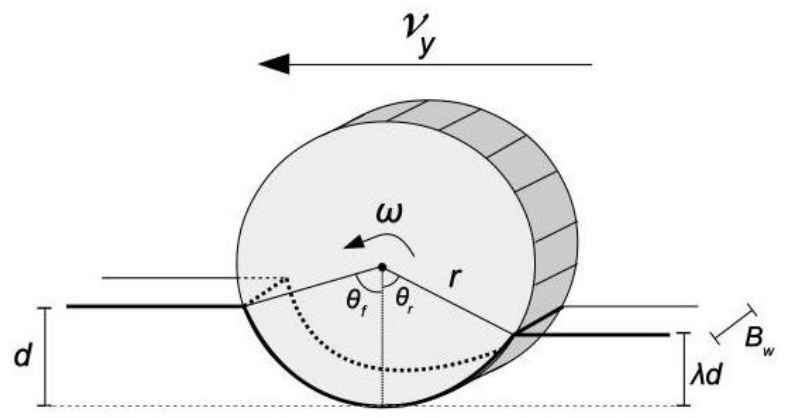

Figure 1.7 Schematic figure of a wheel with radius $r$ rotating at angular velocity $\omega$ and moving forward at $v_{y}$.

Furthermore, the slip angle $\beta$ can be used to quantify the slip in the lateral direction (perpendicular to the longitudinal direction of commanded travel). This angle represents the deviation of the actual velocity with respect to the orientation of the commanded velocity and is given as

$$
\beta=\tan ^{-1}\left(\frac{v_{x}}{v_{y}}\right),
$$

where $v_{x}$ and $v_{y}$ are the vehicle's linear velocities expressed along the vehicle $X$ and $Y$ axis respectively (see Fig. 1.3). Previous work pointed out that, for skid-steer rovers, as the turning radius decreases, the slip angle increases as does the power consumption and motor torque required [15]. 
There has also been previous related work on predicting the rover's slip by visually identifying the terrain class and its geometry. Prior to predicting the slip, an extensive testing phase was done in the Jet Propulsion Laboratory Mars Yard to relate slip and slope angle for different terrains (loose sand, consolidated sand and bedrock) [16]. The resulting slip curves provided a decent estimate of the overall slip on a slope but did not separate lateral and longitudinal slip and assumed that the slip vector was always pointing directly down-slope. To correct the overestimation of the lateral slip, other work used local measurements with Gaussian Process regression to adapt and improve the slip model [17]. In this approach, lateral and longitudinal slip were treated separately and assumed to be a function of only roll and pitch respectively. This considerably improved the slip prediction, especially in high slip areas.

\section{Sinkage}

Wheel sinkage is often divided into static and dynamic sinkage. The static sinkage depends on the vertical load applied to the wheel whereas the dynamic sinkage comes from the rotation of the wheel as it depends on slip ratio, wheel surface pattern, and soil characteristics [14]. First, the static sinkage can be estimated numerically by solving Eq. 1.24 knowing the vertical load $p$ applied to the wheel. The $\theta_{s}$ angle is equal to the front and rear entrance angle of the static wheel, i.e. $\theta_{s}=\theta_{f}=\theta_{r}$ (see Fig. 1.7) when $v_{y}=0 \mathrm{~m} / \mathrm{s}$ and $\omega=0 \mathrm{rad} / \mathrm{s}$. Following the static stress formulation by Bekker et al. [18], the vertical load applied to a wheel is defined as

$$
p=r^{n_{s}+1}\left(k_{c}+k_{\phi} B_{w}\right) \int_{-\theta_{s}}^{\theta_{s}}\left(\cos \theta_{w}-\cos \theta_{s}\right)_{s}^{n} \cos \theta_{w} d \theta_{w}
$$

where $k_{c}$ and $k_{\phi}$ are respectively the cohesive and frictional modulus of the soil, $n_{s}$ is the sinkage exponent, $B_{w}$ is the thickness of the wheel, and $\theta_{w}$ is an arbitrary angle along the wheel. Accordingly, knowing the $\theta_{s}$ angle, the static sinkage is obtained following:

$$
d_{0}=r\left(1-\cos \theta_{s}\right)
$$

Another way to estimate the static sinkage of a wheel is by using a bevameter to experimentally obtain the sinkage parameters, i.e. $k_{c}, k_{\phi}$ and $n_{s}$ [9]. Based on Bekker's pressure-sinkage equation, these parameters are then used to estimate the static sinkage of the wheel following:

$$
d_{0}=\left[\frac{3 p}{B_{w}\left(3-n_{s}\right)\left(k_{c} / B_{w}+k_{\phi}\right) \sqrt{2 r}}\right]^{\left(2 / 2 n_{s}+1\right)} .
$$

On the other hand, the dynamic sinkage for a wheel moving on loose soil is typically harder to model due to the complexity of the interactions between the wheel and deformable soil. The 
current dynamic sinkage models are strongly dependant on the longitudinal slip ratio $s_{y}$ defined in Eq. 1.22. For instance, Lyasko wrote in [19] that Reece proposed in [20] that a wheel sinkage could be modeled using:

$$
d=d_{0}+h \frac{s_{y}}{1-s_{y}},
$$

where $d_{0}$ is the static sinkage and $h$ is the grouser height. The second term models the additional sinkage due to shear deformation. In this model, the wheel sinks by the height of the grousers at $50 \%$ slip and by infinity at $100 \%$ slip. The work of [19] then presents a similar model developed by Vasil'ev [21] which multiply the longitudinal slip $s_{y}$ by an experimentally determined constant $\left(H_{p}\right)$ :

$$
d=d_{0}+s_{y} H_{p}
$$

From this model, Lyasko proposed the following equation to model the dynamic sinkage of a wheel with a static sinkage of $d_{0}$ and slipping by $s_{y}$ :

$$
d=\frac{1+s_{y}}{1-0.5 s_{y}} d_{0} .
$$

In this model, a $0 \%$ and $100 \%$ slip case would result in the sinkage value of $2 d_{0}$ and $4 d_{0}$, respectively. It is noticed that there isn't a large variation in the sinkage estimation as the slip ratio increases.

Ding et al. [22] later proposed a new sinkage model based on improved normal and shear stress models introduced in [23] and [24] respectively. Knowing the wheel load and slip ratio $s_{y}$, a set of equations are evaluated to obtain the front entrance angle $\theta_{f}$ (seen in Fig. 1.7). This angle is then used in the following equation to estimate the wheel sinkage:

$$
d=r\left(1-\cos \theta_{f}\right)+h s_{y}-\Delta d_{4},
$$

where $\Delta d_{4}$ is a constant determined experimentally. Another terramechanic model using the dynamic pressure-sinkage relationship [25] was examined but not considered further due to the complexity of the model.

All the sinkage models presented are strongly dependant almost exclusively on the longitudinal slip and do not take into account the effect of the lateral slip/skidding of the wheel while turning. The models were all validated using a single-wheel test bed controlling the commanded and actual velocity in the longitudinal direction, which isn't a fair comparison to a sinkage experienced by a skid-steer rover performing a turn. Most importantly, these slip-sinkage models only provide an estimate of the steady-state value of the wheel sinkage under a slip ratio of $s_{y}$. 
Finally, as seen in Fig. 1.7, it should be noted that the sinkage $d$ is the sinkage recorded in front of the wheel, as the sinkage at the back is influenced by the normal load, soil characteristic, wheel surface pattern an slip ratio. The relation between the front and rear sinkage is denoted using the wheel sinkage ratio $\lambda$. This ratio is smaller than 1 when the soil is being compacted by the load of the wheel but can also be greater than 1 when the soil under the wheel is being excavated to the region behind the wheel [14].

\section{Sand Bulldozing Force}

A wheel skidding laterally through soil/sand may cause an accumulation of sand on the side of the wheel that adds to turning resistance. This resistance is analogous to that experienced when bulldozing soil with a blade.

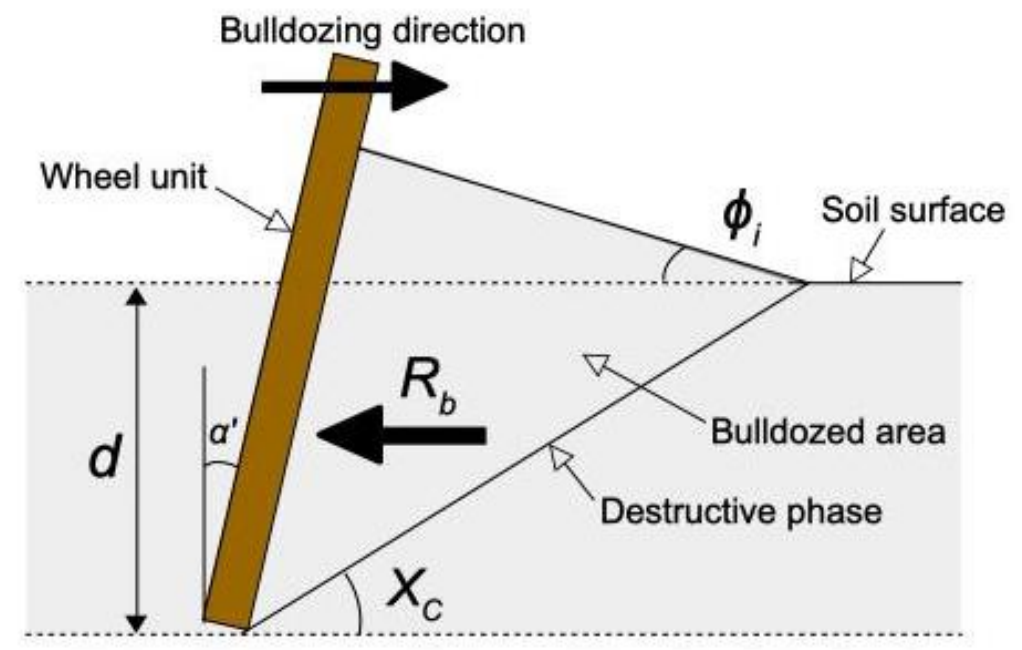

Figure 1.8 Estimation model of the bulldozing resistance by Bekker et al. [26] used by Ishigami et al. [14] to compute the sand resistance next a skid-steer rover's wheel

The concept of bulldozing resistance was initially presented in 1960 by Hegedus [27] for a blade moving into soil. Ishigami et al. [14] later used Hegedus' estimation of the bulldozing resistance and Bekker et al. [26] approximation of the destructive angle $X_{C}$ (defined in Eq. 1.35) to define the bulldozing resistance force acting on the side of the wheels of a skid-steer rover. Knowing the wheel radius $r$ and the sinkage $d\left(\theta_{w}\right)$ at an arbitrary angle along the wheel $\theta_{w}$, the reaction force generated by the bulldozing resistance, i.e. $F_{S}$, is written as follow:

$$
F_{s}=\int_{\theta_{r}}^{\theta_{f}} R_{b}\left(r-d\left(\theta_{w}\right) \cos \theta_{w}\right) d \theta_{w}
$$

where $\theta_{f}$ and $\theta_{r}$ are the entry (shown in Fig. 1.7) and exit angles respectively and $R_{b}$ is the bulldozing resistance. This resistance is calculated as a function of $d\left(\theta_{w}\right)$ : 


$$
R_{b}(h)=D_{1}\left[c d\left(\theta_{w}\right)+D_{2} \frac{\rho d^{2}\left(\theta_{w}\right)}{2}\right]
$$

where

$$
D_{1}=\cot X_{c}+\tan \left(X_{c}+\phi_{i}\right), \quad \text { (1.33) } \quad D_{2}=\cot X_{c}+\cot ^{2} X_{c} / \cot \phi_{i}
$$

$c$ is the cohesion stress of the soil, $\rho$ is the soil dry bulk density and $\phi_{i}$ is the internal friction angle. Following Bekker's approximation, the destructive angle is defined as:

$$
X_{C}=45^{\circ}-\phi_{i} / 2
$$

As it can be seen in this set of equations, the added force caused by the sand accumulation next to a wheel remains constant with respect to the wheel displacement, assuming a constant wheel sinkage. This means that the model doesn't capture the effect of the accumulation of sand next to a wheel as it is moving during a turn.

Another related background work is the model developed by Skonieczny [28] to model the effect of surcharge accumulation on a wide-blade soil-tillage tool. This model is based on the principles of passive earth pressure and explicitly ignores inertial forces, due to the low cutting speed assumption. Using a set of soil parameters such as the soil dry bulk density $\rho$, the soil cohesion $c$ as well the internal and external friction angles ( $\phi_{i}$ and $\delta$ respectively), the algorithm first finds the destructive angle $\left(\alpha_{2}\right)$ value that minimize the excavation force, namely $F^{E X}$, at each cut advance distance $x . F^{E X}$ is defined as follow:

$$
F^{E X}=w\left(\begin{array}{r}
\frac{\frac{1}{2} \rho g d r_{\alpha}+\rho g r_{q}^{2} \tan \phi_{i}-I_{\alpha_{1}} \rho g \frac{\left(r_{q}-d \cot \alpha_{1}\right)^{2}}{2\left(\cot \phi_{i}+\cot \alpha_{1}\right)}-I_{\alpha_{2}} \rho g \frac{\left(r_{q}-d \cot \alpha_{2}\right)^{2}}{2\left(\cot \phi_{i}+\cot \alpha_{2}\right)}}{\cos \left(\alpha_{1}+\delta\right)+\sin \left(\alpha_{1}+\delta\right) \cot \left(\beta+\phi_{i}\right)} \\
\left.+\frac{c\left(d+h_{f}\right)\left[1+\cot \beta \cot \left(\beta+\phi_{i}\right)\right]}{\cos \left(\alpha_{1}+\delta\right)+\sin \left(\alpha_{1}+\delta\right) \cot \left(\beta+\phi_{i}\right)}\right),
\end{array}\right.
$$

where $w$ is the width of the blade, $d$ is the cut depth (assumed constant), $\alpha_{1}$ is the rake angle, $g$ is the gravitational acceleration, $r_{\alpha}$ is the horizontal distance at soil level from the blade to the front of the failure plane, $h_{f}$ is the addition height that the shear plane (the dotted line in Fig 1.9) needs to pass through and $I_{\alpha_{1}}, I_{\alpha_{2}}$ are Boolean indicator functions:

$$
I_{\alpha_{1}}=\left\{\begin{array}{ll}
1, & r_{q}>d \cot \alpha_{1} \\
0, & r_{q} \leq d \cot \alpha_{1}
\end{array} \quad(1.37) \quad I_{\alpha_{2}}= \begin{cases}1, & r_{q}>d \cot \alpha_{2} \\
0, & r_{q} \leq d \cot \alpha_{2}\end{cases}\right.
$$


The equation of the added height, $h_{f}$, and the horizontal distance between the front and the peak of the sand pile, $r_{q}$, can be found in [28].

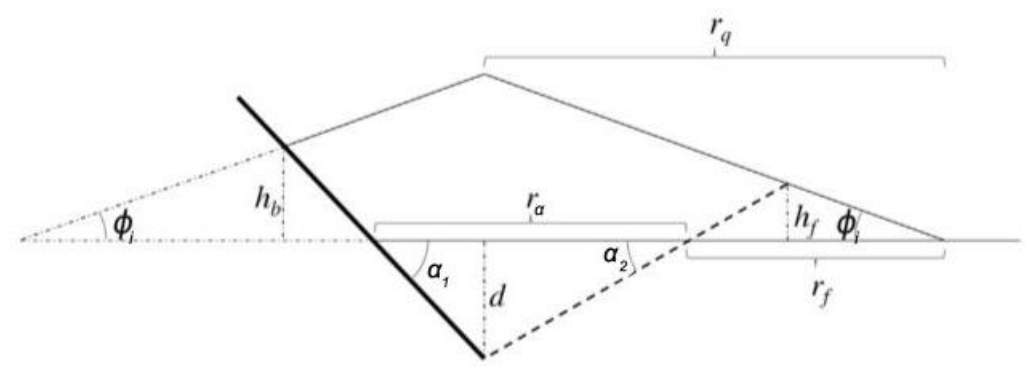

Figure 1.9 Geometry of the soil cutting problem used by Skonieczny [28]. $\alpha_{2}$ represents the destructive angle, which is denoted $X_{C}$ in this work.

As the wheel progresses during a turn, the cut advance distance $x$ will also increase. Accordingly, the excavation force $F^{E X}$ will increases as the turn progresses, hence modeling properly the accumulation of sand next to the wheels of a skid-steer rover. The model presented by Skonieczny should then be simplified and adapted to the case of a skid-steer rover wheel progressing in a turn, i.e. the sinkage $d$ is not constant and $\alpha_{1}$ can be assumed as $90^{\circ}$. The simplification of this model along with the relevant assumptions for the case of a wheel bulldozing the sand will be shown in Section 5.4 .

\subsection{Contributions}

The first main contribution of this research is to show the limitations of a popular hard ground power model for modeling skid-steered wheeled robots operating on loose soil. This limitation was exposed through a comprehensive experimental assessment of the average and maximum power consumed during turns of varying radii on two different types of sand, with two skid-steered wheeled rover platforms.

The second main contribution is the identification of important phenomena contributing to the increased power consumption of skid-steer rovers on loose soil, in particular in the range of turning radii where the inner wheels rotate backwards but are being dragged forward by the outer wheels. This non-efficient method of driving (but necessary in order to achieve turns of particular radii) results in added wheel sinkage but also in increasing wheel torque to excavate the sand. A power model for skid-steer rovers on loose soil would need to model these two phenomena to accurately estimate the power consumption for any turns.

The concept of angular slip is the third main contribution of this work. Similarly to the longitudinal slip found in the literature, this new concept takes the actual and commanded angular 
velocity to quantify to difference between the actual and expected time to complete a turn. Using experimental data, it is shown that there is a significant difference in the recorded angular slip on hard ground and loose soil, and that the angular slip peaks at $R^{\prime}$, the turning radius where the inner wheels are not commanded to turn. An estimation of the angular slip for a particular soil allow us to predict the time to complete a turn and thus help when finding energy-efficient paths, as energy is the integral of power over time.

\subsection{Thesis Outline}

In this thesis, the power consumption of a four-wheeled skid-steered robot is studied on various terrains. As seen in Fig. 1.10, two terrain types, i.e. sand and hard ground, and two terrain geometry, i.e. flat and sloped, are considered but only the power consumed in sand is examined when looking at the sloped terrain geometry. For hard ground, sloped hard terrain was already extensively covered in the literature by the work of [7] and thus was not considered within the scope of this thesis. On the other hand, this research goes further than the current state of power modeling of skid-steer rovers on flat ground, both on hard ground and on sand. It should be kept in mind that this study of power consumption on these terrains is in the goal of finding energyefficient paths. Therefore, the time dependency of the power is also considered and a new concept of angular slip is presented in order to explain the discrepancies of the results on hard ground and loose soil, when comparing the total energy during two types of path: circle-line-circle (CLC) and point turn-line-point turn (PLP).

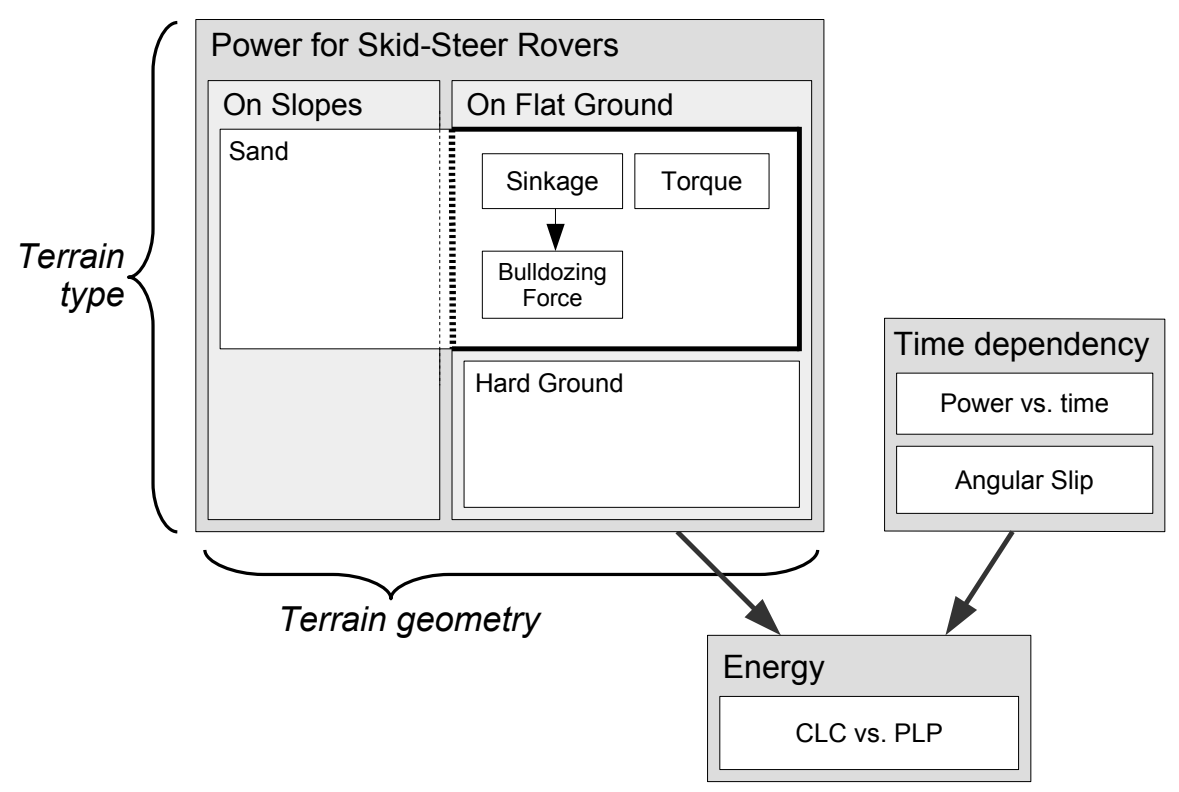

Figure 1.10 High level overview of the topics studied in this research 
The remainder in this thesis is divided as follow. Chapter 2 shows the work done to simplify both the kinematic equations and ICR-based power model, using relevant assumptions and constraints. Chapter 3 then presents the test equipment that is used to evaluate the strengths and limitations of the existing power model, the results of which are shown in Chapter 4. Afterwards, Chapter 5 investigates the contributing factors to increased power when the skid-steer rover is operating on loose terrain, instead of the typically considered hard terrain. Chapter 6 then shows the energy comparison of the two types of path aforementioned, providing additional insights on a new concept to consider when planning an energy-efficient path on loose soil. Finally, Chapter 7 summarizes the main conclusions of this research and discusses future work. 


\section{Chapter 2}

\section{Power Modeling of Skid-Steered Wheeled Robots}

In this chapter, the kinematic model presented in Section 1.1.1 is simplified using appropriate assumptions and constraints. In the process, a novel velocity constraint is selected and justified, and a special set of turning radii is illuminated. Then, the ICR-based power model from the literature presented in 1.1.3 is simplified using the updated kinematic model.

Throughout this work, the $R^{\prime}$ turning radius is identified as particularly important. When a skid-steered wheeled robot is following a circular arc path with radius $R^{\prime}$, the inner wheels are not commanded to turn. This means that the inner wheel commanded velocity is negative when the turning radius, i.e. $R$, is smaller than $R^{\prime}$ and the inner wheel commanded velocity is positive when $R>R^{\prime}$

\subsection{Simplifying the Kinematic model}

As seen in the literature, the location of the center of mass can have an impact on the location of the instantaneous centers of rotation and hence on power modeling. To simplify the problem, the center of mass is assumed to be at the rover's geometric center. As this implies a symmetry in the left and right ICR locations, i.e. $x_{I C R_{r}}=-x_{I C R_{l}}$, Eq. 1.5 can be simplified to:

$$
v_{y}=\frac{V_{r}+V_{l}}{2} .
$$

Also, Eq. 1.7 is repeated:

$$
\omega_{z}=\frac{V_{r}-V_{l}}{B_{s}}
$$


As the rover is operating at low speed, the lateral slip is neglected, i.e. $v_{x}=0$. With this assumption, the $\vec{v}$ velocity is therefore equal to $v_{y}$. It is well known that if $v_{y}$ is tangential to the arc of rotation, then

$$
v_{y}=\omega_{z} R,
$$

where $R$ is the turning radius of the robot, depicted in Fig. 1.3. Accordingly, the turning radius $R$ can be defined as a function of $V_{r}, V_{l}$ and $B_{s}$. For simplicity, only the absolute value of the turning radius is treated in this work. Since symmetry is assumed, without loss of generality, all the turns can be considered as left turns with radius $R$, as depicted in Fig. 1.3.

$$
R=\frac{B_{s}}{2}\left|\frac{V_{l}+V_{r}}{V_{l}-V_{r}}\right|
$$

The $R^{\prime}$ radius can now be defined as the turning radius where the inner wheel's commanded linear velocity is zero. Using Eq 2.4, we can find the value of $R^{\prime}$ with either $V_{l}=0$ or $V_{r}=0$ :

$$
R^{\prime}=\frac{B_{s}}{2}
$$

\subsubsection{Specifying the Velocity Constraint}

An important constraint considered in this work is that the total absolute velocity $v_{c}$ of the rover is constant. This total absolute velocity is defined as

$$
v_{c}=\frac{\left|V_{r}\right|+\left|V_{l}\right|}{2} .
$$

Note that for $R \geq R^{\prime}, V_{r}$ and $V_{l}$ are both positive, and thus the constant $v_{c}$ assumption is equal to constant forward velocity (Eq. 2.1). For $R<R^{\prime}$, on the other hand, $V_{r}$ and $V_{l}$ are of opposite sign, thus the constant $v_{c}$ assumption is equal to constant angular velocity (Eq. 1.7 or 2.2).

Many works on rover power consumption assume constant forward velocity [10][11][12]. However, the stated constraint of total absolute velocity is more realistic for skid-steered rovers than a constant forward velocity $\left(v_{y}=v_{\text {const }}\right)$ constraint, for example. As turning radius approaches zero, constant forward velocity would require angular velocity, and $V_{l}$ and $V_{r}$, to approach infinity. In real systems, motors producing $V_{l}$ and $V_{r}$ saturate at a maximum value. One could argue that the constant forward velocity constraint could be enforced by allowing the left and right velocity 
control inputs to saturate at $v_{\max }$. However, this results in a lower bound on achievable turning radius, $R_{\text {min }}$. Starting from Eq. 2.4, for a left turn, when $V_{r}=v_{\max }$, the minimum turning radius $R_{\text {min }}$ would be:

$$
R_{\text {min }}=\frac{B_{s}}{2}\left|\frac{v_{\text {const }}}{v_{\text {max }}-v_{\text {const }}}\right| \neq 0 \text { for } v_{\text {const }}>0
$$

Thus, if the complete range of turning radii is to be explored, the stated $v_{c}$ constraint is more realistic than one of constant forward velocity $v_{\text {const }}$, even with an added $v_{\max }$ upper linear velocity bound.

Looking at the total absolute velocity $v_{c}$ constraint defined by Eq. 2.6, it can now be expressed using a piece-wise function knowing that, for a left turn (where $V_{r}>0$ always), $V_{l} \leq 0$ when $0 \leq R \leq R^{\prime}$ and $V_{l}>0$ when $R>R^{\prime}$. As $\left|V_{l}\right|=V_{l}$ when $V_{l}$ is positive, and $\left|V_{l}\right|=-V_{l}$ when $V_{l}$ is negative, Eq. 2.6 is rewritten as:

$$
v_{c}=\left\{\begin{array}{ll}
\frac{V_{r}-V_{l}}{2}, & 0 \leq R \leq R^{\prime} \\
\frac{V_{r}+V_{l}}{2}, & R>R^{\prime}
\end{array} .\right.
$$

Using Eq. 2.2, Eq. 2.3, and this new $v_{c}$ definition, the angular velocity can then be rewritten as a function of $v_{c}$ and $R$. This equation will be useful in Section 2.2 when simplified the ICR-based power model presented in Section 1.1.3.

$$
\omega_{z}= \begin{cases}\frac{2 v_{c}}{B_{s}}, & 0 \leq R \leq R^{\prime} \\ \frac{v_{c}}{R}, & R>R^{\prime}\end{cases}
$$

The forward linear velocity $v_{y}$ can also be rewritten to:

$$
v_{y}=\left\{\begin{array}{ll}
\frac{2 R}{B_{s}} v_{c}, & 0 \leq R \leq R^{\prime} \\
v_{c}, & R>R^{\prime}
\end{array} .\right.
$$

These equation support the claim that the total absolute velocity constraint results in constant angular velocity $\omega_{z}$ when $0 \leq R \leq R^{\prime}$ and in constant forward velocity $v_{y}$ when $R>R^{\prime}$. The top plot of Fig. 2.1 shows the $v_{y}$ and $\omega_{z}$ values for a left turn, when $v_{c}=0.3 \mathrm{~m} / \mathrm{s}$. The bottom plot presents the corresponding left and right velocities, showing that, at $R^{\prime}$, the inner $\left(V_{l}\right)$ and the outer $\left(V_{r}\right)$ wheel commanded velocities are respectively zero and $2 v_{c}$. Then, as $R$ increases, the left and right velocities approach $v_{c}$ to eventually reach it at $R=\infty$, when the rover is moving along a straight line. Appendix A shows the derivation of the equations used to draw the curves of Fig. 2.1. 

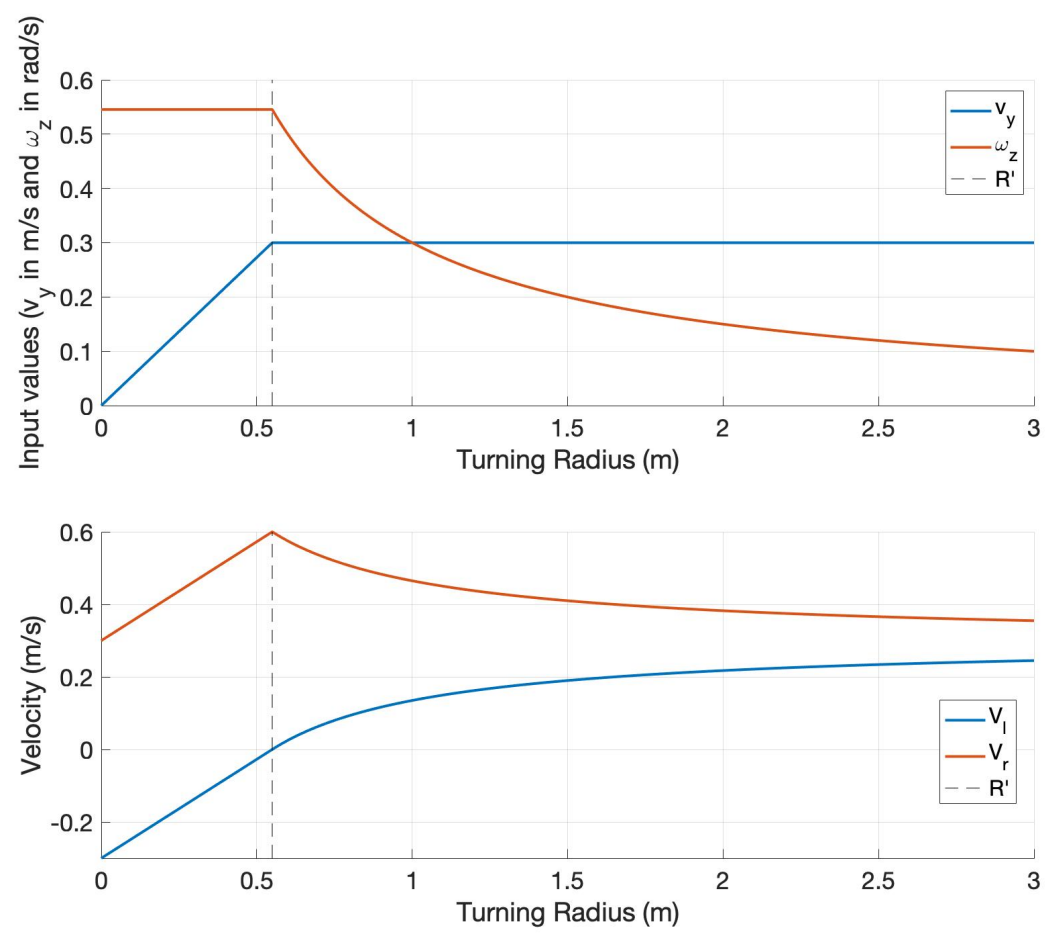

Figure 2.1 Resulting linear velocity $v_{y}$ and angular velocity $\omega_{z}$ (top), and corresponding left and right commanded linear velocities, i.e. $V_{l}$ and $V_{r}$ respectively, as a function of the commanded turning radius, for a left turn, under a $v_{c}=0.3 \mathrm{~m} / \mathrm{s}$ constraint. The constant $v_{c}$ constraint is defined in Eq. 2.6.

\subsubsection{Special Case $\left(B / 2<R<R^{\prime}\right)$}

A further analysis of the skid-steered wheeled robot kinematics, when considering the slip track $B_{s}$, leads to an important fact about a particular set of turning radii. When the rover is moving along a circular arc path with a radius that is slightly bigger than half of its width $(B / 2)$ but smaller than $R^{\prime}$, the inner wheels are rotating backward but are actually being dragged forward by the outer wheels. Fig. 2.2 shows the actual and commanded velocity vectors of the inner wheel ( $v_{i}^{A C T}$ and $v_{i}^{C M D}$ respectively) when the rover is dealing with this set of turns.

In this work, the slip angle $\beta$ is defined as being the angle between $v^{A C T}$ and $v^{C M D}$. This is a generalization of the definition found in the literature as Eq. 1.23 is equivalent to the angle between $v^{A C T}$ and $v^{C M D}$ (i.e. the vehicle's $Y$-axis), if $\beta<90^{\circ}$ is assumed. When $v^{C M D}=0$, which is an exception only occuring at $R^{\prime}$, the slip angle should be defined as the smallest angle between $v^{A C T}$ and the vehicle's $Y$-axis. Using this definition, the inner wheels' slip angle $\left(\beta_{i}\right)$ will always be strictly greater than $90^{\circ}$ for the identified special case. It should be noted that a slip angle is always present when turning skid-steered wheeled robots as the wheels are fixed in orientation but 
lateral movement is required to achieve arcs. However, slip angles greater than $90^{\circ}$, where the actual velocity has a component opposing the commanded velocity is unique to the special case described here for the first time.

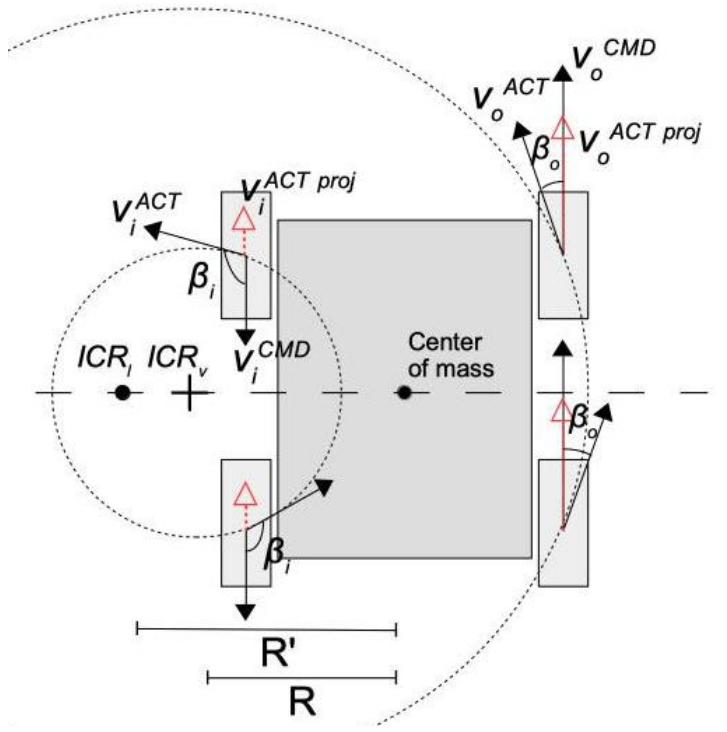

Figure 2.2 Schematic figure of a four-wheel skid-steered rover performing a turn of radius $B / 2<$ $R<R^{\prime}$. For the inner wheels, the projection of tangential velocity (i.e. actual velocity of the wheel $v^{A C T}$ ) onto the axis of the commanded wheel velocity, $v^{C M D}$, is opposite in direction to $v^{C M D}$.

It should be noted that, going forward, the experiments are done with left hand turns, such that $V_{l}=v_{i}^{C M D}$ and $V_{r}=v_{o}^{C M D}$. The power consumption and the inner wheel sinkage during a left hand turn with a radius in the special case range will be presented and discussed in Section 4.4 and 5 respectively.

\subsection{Simplifying the ICR-Based Power Model}

In this section, the power model based on instantaneous centers of rotation presented in Section 1.1.3 is simplified using the assumptions and constraints stated above. First, if the rover is symmetric both in $X$ and $Y$ (Fig. 1.3), the distances $d_{I C R}=\left\|\vec{a}-\vec{C}_{n}\right\|$ are equal and constant for all wheels. Then, assuming small rover pitch angle $\left(|\theta| \leq 15^{\circ}\right)$ in a sloped environment, i.e. $\cos \theta \approx 1$ and $\sin \theta \approx \theta$, the sum of the normal forces $p$ is constant such that

$$
p=p_{1}+p_{2}+p_{3}+p_{4}=m g,
$$

where $p_{1}, p_{2}, p_{3}$ and $p_{4}$ are the normal forces acting on the front left, front right, back left and back right wheel respectively. 
Combining these simplifications with the piece-wise angular velocity definition (Eq. 2.9), the power model defined in Eq. 1.16 can be simplified to

$$
P= \begin{cases}\frac{2 c_{1}}{B_{s}}+c_{2}+c_{3} \theta, & 0 \leq R \leq R^{\prime} \\ \frac{c_{1}}{R}+c_{2}+c_{3} \theta, & R>R^{\prime}\end{cases}
$$

where $c_{1}, c_{2}$ are constants and defined as follow:

$$
c_{1}=\mu v_{c} m g d_{I C R}
$$

$$
c_{2}=2 G v_{c}
$$

and the $c_{3}$ term has a $v_{y}$ dependency and is defined as follow:

$$
c_{3}=\Phi m g v_{y}
$$

Since the constant $v_{c}$ constraint is equivalent to a constant forward velocity for turns with $R>R^{\prime}$, the $c_{3}$ value is constant for turns larger than $R^{\prime}$. Note that although Eq. 2.12 simplifies $\sin \theta$ to $\theta$ in the third term by way of the small angle assumption, the $c_{3}$ term is taken directly from Eq. 1.16 without any assumptions of rover symmetry required.

\section{On Flat Terrain}

When the skid-steer rover is operating on flat terrain, i.e. $\theta \approx 0^{\circ}$, the power model can be reduce to:

$$
P=\left\{\begin{array}{l}
\frac{2 c_{1}}{B_{s}}+c_{2} \text { if } 0 \leq R \leq R^{\prime} \\
\frac{c_{1}}{R}+c_{2} \text { if } R>R^{\prime}
\end{array}\right.
$$

As it can be seen in Eq. 2.16 and illustrated in Fig. 2.3, when operating on flat terrain under a constant $v_{c}$, the modeled power remains constant for turning radii smaller than $R^{\prime}$. This will be validated on hard ground in Section 4.3. On the other hand, it will be shown in Section 4.4 that this prediction is inconsistent with observed results on loose sandy soil. 


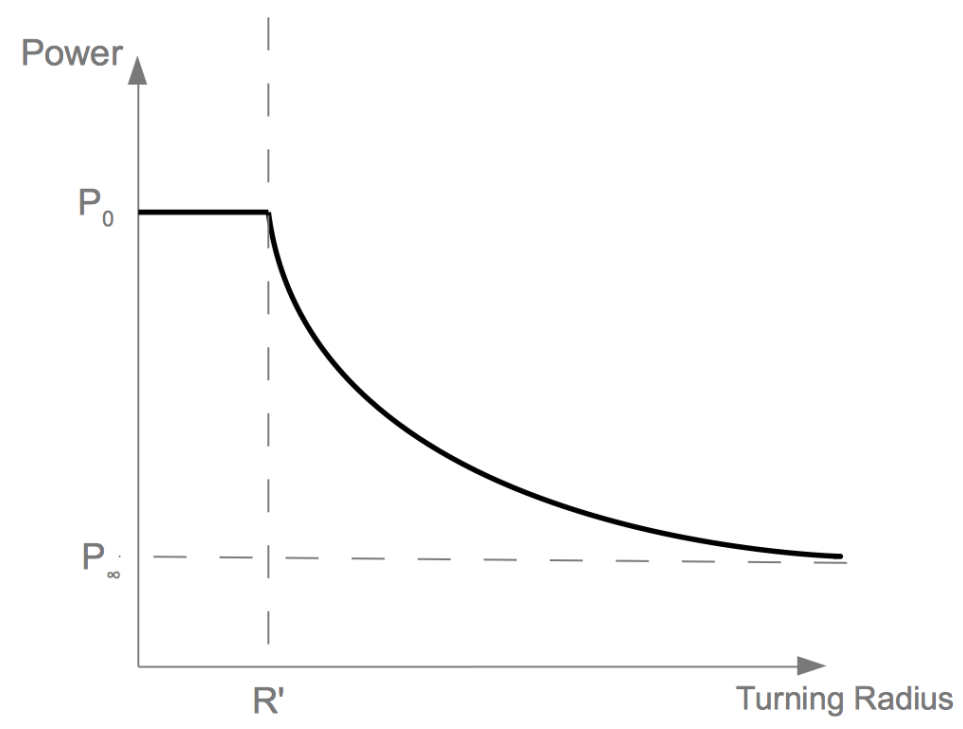

Figure 2.3 Predicting power vs. turning radius using Eq. 2.16 on flat terrain. Comparison with the experimental results on hard ground (Fig. 4.6b) sows concurrence and comparison with the experimental results on sandy soil (Fig. 4.10) shows the limitation of the existing model.

\subsection{Summary of Assumptions and Constraints}

To summarize, here are the main assumptions and constraints that are used throughout this work:

- The total absolute velocity $v_{c}$, defined in Eq. 2.6, is constant.

- The skid-steer rover's center of gravity is located at its geometric center. This implies symmetry in the location of the instantaneous centers of rotation.

- In the sloped environment, the pitch angle of the rover is relatively small, i.e. $|\theta|<15^{\circ}$.

- As the rover is operating at low speeds, the vehicle lateral slip is neglected, i.e. $v_{x}=0$. 


\section{Chapter 3}

\section{Test Equipment and Setup}

For the purpose of this research, two popular skid-steered wheeled robots were used to study the power consumption of this type of rover on sand: the Argo J5 and the Clearpath Husky A200. These rovers were operated on three different test sites: the controlled laboratory sandbox, the Mars analogue terrain of the Canadian Space Agency and at the White Sand National Monument in New Mexico, USA.

This chapter first provides an overview of the Argo J5, the Husky A200 and a single-wheel test bed used for further experimentation. Section 3.4 then provides important insights on the three different test sites and their particularities. As will be explained thoroughly, efforts were made to limit the soil disturbance and improve the repeatability of the tests. Cone penetrometer readings were taken to measure the density but also the consistency of the soil after the sand preparation.

\subsection{Argo J5}

The Argo J5 is an extreme-terrain vehicle produced by Ontario Drive and Gear (ODG). This commercial version of a rover configuration developed for the Canadian Space Agency (CSA) has a large payload capacity, a low center of gravity and is easily customizable as it is equipped with a computer running the Robotic Operating System (ROS). The J5 is symmetric along its left/right (i.e. $X$ ) axis, but not along its front/back (i.e. $Y$ ) axis. 


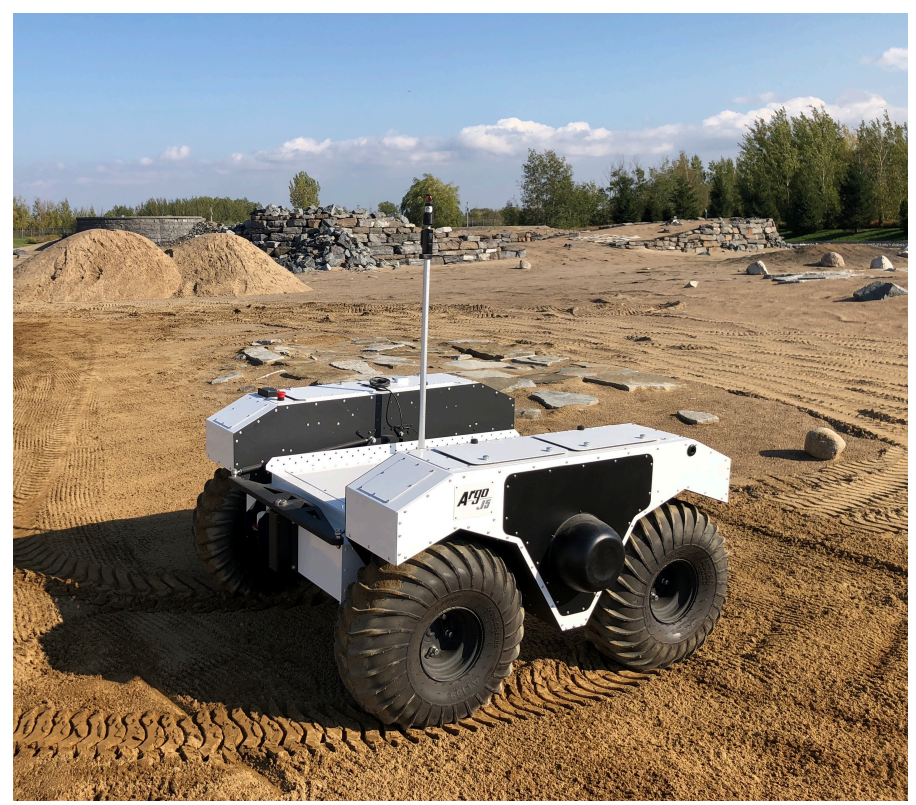

Figure 3.1 Argo J5 (mounted with a landmark prism and an IMU) at the Canadian Space Agency Mars analogue terrain.

The J5 was first brought to the Mars analogue terrain of the Canadian Space Agency to perform turns on sand slopes ranging from 0 to 10 degrees. The current consumption of the left and right motor driver was measured through the built-in ROS power measurement system and the vehicle's pitch $(\theta)$, roll $(\phi)$ and yaw $(\psi)$ were measured using a VectorNav VN-100R inertial measurement unit (IMU). The data recorded from these tests was then used to characterize the impact of the skid-steered rover's pitch and roll on the total power consumption and the results are shown in Section 4.1. Figure 3.1 shows the Argo J5 performing a turn at the Mars analogue terrain and Table 3.1 includes the important geometric parameters and mass of the rover.

Table 3.1 Geometric parameters of the Argo J5 platform

\begin{tabular}{ll}
\hline Parameter & Value \\
\hline Track Width $(B)$ & $1.14 \mathrm{~m}$ \\
Defined Slip Track $\left(B_{S}\right)$ & $1.14 \mathrm{~m}$ \\
Length $(L)$ & $0.98 \mathrm{~m}$ \\
Wheel radius $(r)$ & $0.3 \mathrm{~m}$ \\
Mass $(m)$ & $372 \mathrm{x}$ \\
\hline
\end{tabular}




\subsection{Clearpath Husky A200}

The main platform used to experimentally investigate the existing power model is the Clearpath Husky A200. This medium-sized skid-steered wheeled robot is fully supported in ROS. Although this rover has a built-in power measurement system, our preliminary testing unveiled some inaccuracy in the current measurements. An external board using the Texas Instruments INA226 bidirectional current and power sensor was therefore designed and implemented to measure the current and voltage drawn by the left and right motor drivers and publish the data to a ROS topic. The details of the issue encountered and the USB current sensing board design are presented further below in this section. Furthermore, the orientation of the robot was also recorded using the VectorNav VN-100R rugged IMU and the left and right wheels' commanded velocities were recorded from the built-in ROS system. The position of rover was also tracked using a Leica Viva TS16 Total Station and a landmark prism (reflector) placed along the edge of the rover chassis, at the midpoint along the rover's length (as seen in Figure 3.2a). The distance from the prism to the rover's geometric center is $21 \mathrm{~cm}$.

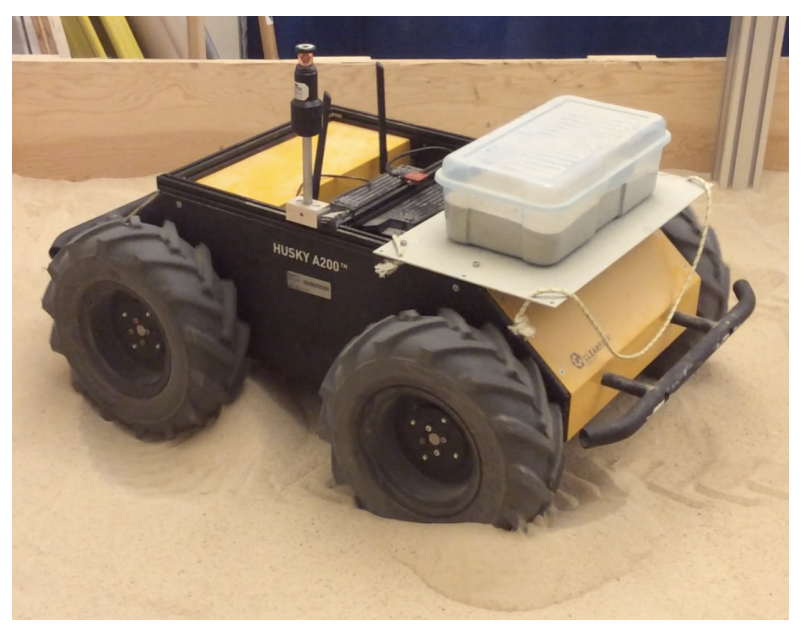

(a)

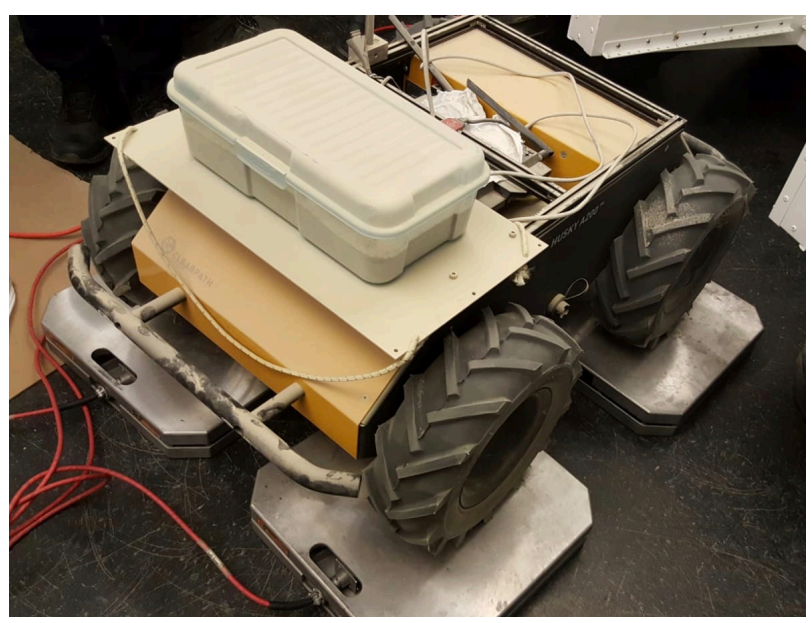

(b)

Figure 3.2 a) Clearpath Husky A200 platform mounted with a landmark prism (for position tracking), an IMU and an additional box of sand to place the center of mass of the rover at its geometric center. b) Clearpath Husky A200 placed on the Computerscales Accuset II portable scale.

For the purpose of our testing, an additional weight was placed at the front of the robot to align the center of mass with the geometric center (as shown in Fig. 3.2a). Knowing that the Husky's battery is located at the back, the four wheels were each placed on a portable car scale (Computerscales Accuset II) to determined the required mass to add on the front wheels axis. Using a box of sand of $8 \mathrm{~kg}$, the rover was weighted again to confirm an equal distribution of 
weight on all four wheels $( \pm 0.5 \%)$. Figure $3.2 \mathrm{~b}$ shows the Husky on the portable car scale with the added box of sand to validate the equal distribution of the weight on the four wheels.

Table 3.2 Geometric parameters of the Clearpath Husky A200 platform

\begin{tabular}{ll}
\hline Parameter & Value \\
\hline Track Width $(B)$ & $0.545 \mathrm{~m}$ \\
Defined Slip Track $\left(B_{S}\right)$ & $1.1 \mathrm{~m}$ \\
Length $(L)$ & $0.52 \mathrm{~m}$ \\
Wheel radius $(r)$ & $0.165 \mathrm{~m}$ \\
Wheel thickness $\left(B_{w}\right)$ & $0.125 \mathrm{~m}$ \\
Mass $(m)$ & $63 \mathrm{~kg}$ \\
\hline
\end{tabular}

The first part of Husky tests consisted of driving the robot on the dunes of the White Sands National Monument (WSNM) in New Mexico, USA (Figure 1.1). This testing area is a large natural terrain consisting of gypsum sand dunes where the rover was manually driven on terrain of varying slope at a nominal speed of $v_{c}=0.3 \mathrm{~m} / \mathrm{s}$. The power measurements recorded during the drives were then fitted based on the turning radius $R$ and the vehicle's pitch angle $\theta$. Results are shown in Section 4.2.

Further tests were conducted in the Concordia Aerospace Robotics Laboratory. The Husky A200 was placed in a $2.2 \times 2.2 \mathrm{~m}$ sandbox and the power consumption of the brushed DC motors was measured for a set of turns of radii close to $R^{\prime}$. These tests were also repeated at the CSA Mars analogue terrain and the results of these two test campaigns are explained in Section 4.4. The data gathered in the laboratory sandbox was used to replicate the inner wheels' trajectory with the five-axis single-wheel test bed while investigating the contributing factors to the increased power consumption on sand presented in Section 5.

The last phase of testing was performed at the CSA Mars analogue terrain as energy consumption of the skid-steered wheeled robot was measured when following two classes of path, i.e. a circle-line-circle (CLC) and a point turn-line-point turn (PLP) path. Further explanation and the results of these tests are presented in Section 6.

\section{External USB Power Measurement Unit}

The power analysis of the preliminary tests unveiled a significant inaccuracy in the current readings from the built-in system. As it can be seen in Fig. 3.3, the measured current of the built-in system reads $0.59 \mathrm{~A}$ even when the rover is not moving. This relatively high base current bias is problematic when the actual motor current consumption is lower than the $0.59 \mathrm{~A}$ threshold. In 
particular on hard ground or when the wheels are rotating at a low speed (i.e. the inner wheels during a turn close to $R^{\prime}$ ), the base current reading results in higher measured power than the actual required power. It was also noticed that their was a significant gap between the left and right current measurements from the built-in system. As seen in the bottom plot of Fig. 3.3, this problem was fixed with our custom USB measurement unit.

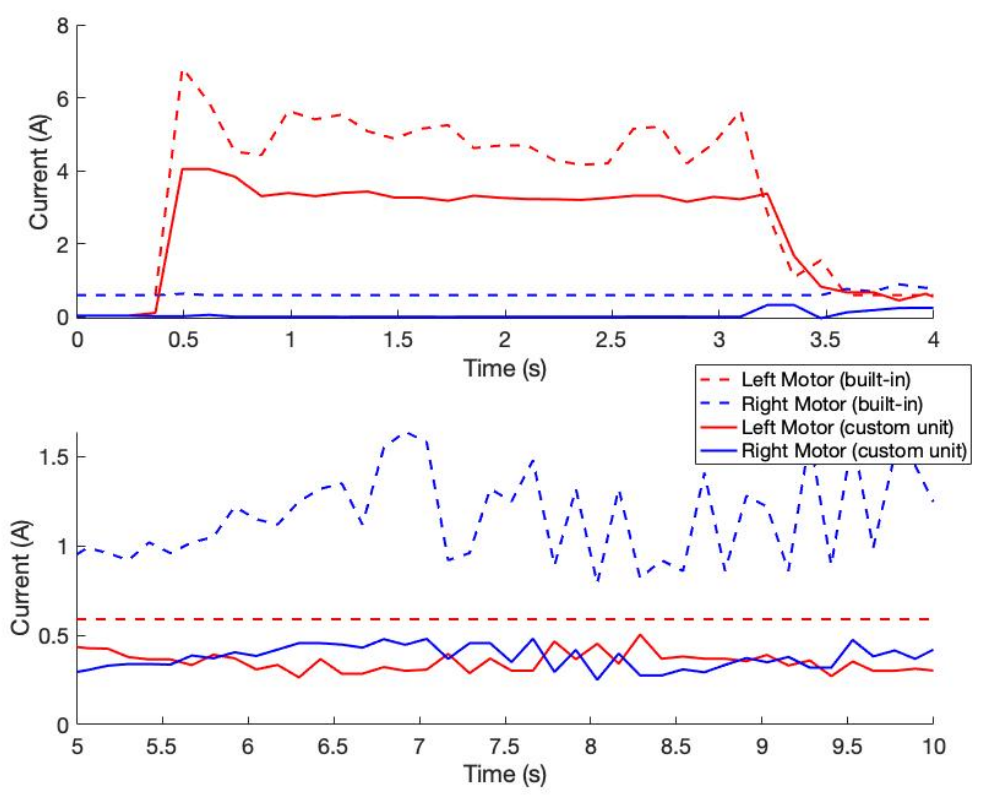

Figure 3.3 Current comparison of the built-in system (dotted line) and custom measurement unit designed for this work (solid line). The left and right current consumption during a right turn of $R=1.5 R^{\prime}$ (top) and during a straight line segment (bottom) on hard ground are shown.

Dogru et al. [13] performed their research using a Clearpath Husky A200 and also noticed inaccurate current measurements. Accordingly, they designed their own power measurement board using the Texas Instrument's INA226 bidirectional current and power sensor chip to solve the problem. In their setup, the current sensor was connected to an Arduino board via $\mathrm{I}^{2} \mathrm{C}$ bus which was polled by the Husky's computer to published the data to a ROS topic. This paper inspired the design of our USB power measurement unit using the INA226 current and power sensor. Instead of using an Arduino board, the FT232H from Future Technology Devices International Ltd (FTDI) was used as interface between the $\mathrm{I}^{2} \mathrm{C}$ and USB bus. This single-channel hi-speed USB to $\mathrm{I}^{2} \mathrm{C}$ (and other communication protocol) was a cheaper option and also allowed us to transfer the data from the printed circuit board (PCB) to the Husky Computer with a USB cable. It should be noted that the left and right motor drivers are placed at the back and at the front of the robot. The custom power units were thus placed next right next to their respective motor driver to limit the noise in the current and voltage measurements and a USB cable was linked back to the computer. 
Fig. 3.4 shows the block diagram and the actual setup of the power measurement unit. The INA226 chip reads the voltage drop across the $5 m \Omega \pm 1 \% 5 \mathrm{~W}$ Through Hole resistor $R_{S H U N T}$ (placed between the battery and the motor driver) and converts it to current using the resistance value stored in the calibration register. The Texas Instrument chip also reads the bus voltage, which corresponds to the battery voltage $V_{B A T T}$. The number of samples averaged and conversion time for the measurements were tuned to obtain smooth data but avoid over-filtering.

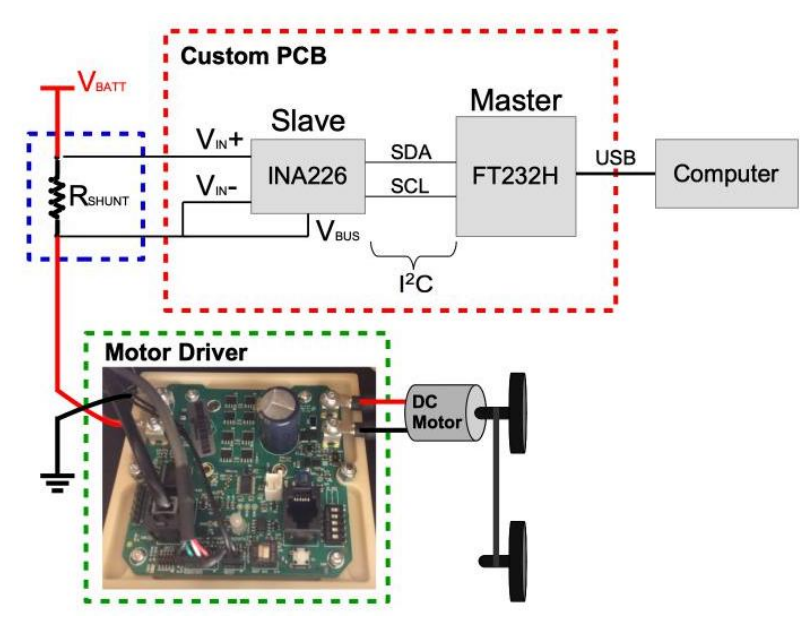

(a)

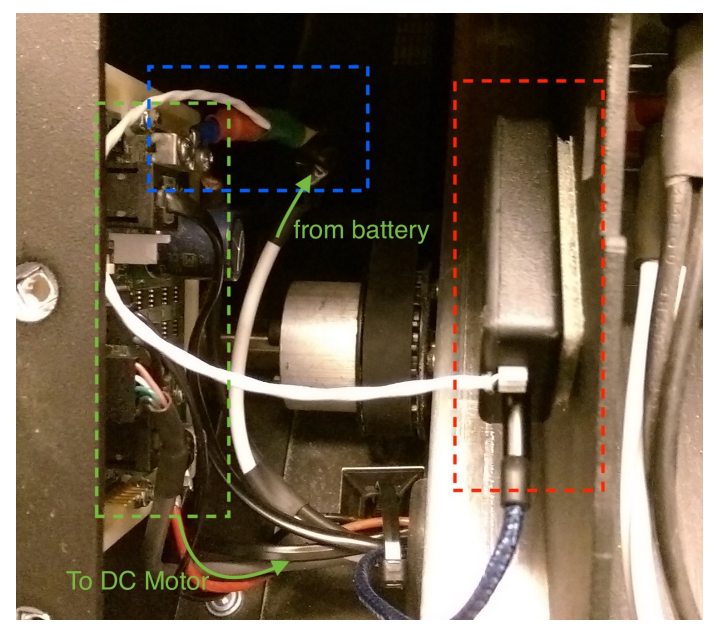

(b)

Figure 3.4 a) Block diagram of the PCB designed and the setup to measure the current fed to the motor driver. b) Custom USB power measurement unit (in red) connected to a shunt sensing resistance (in blue) on the high side of the DC motor driver (in green).

Fig. 3.5 shows one of the two PCBs which were assembled in the laboratory. To differentiate the two units, the slave address of the two INA266 sensor was set to different values using the A0 and A1 pins by physically connecting the two pins to the ground for the left motor driver and to the supply voltage for the right motor driver. The circuit was based on the INA226 and FT232H datasheets and the layout was made to minimize the noise on the $V_{I N}+$ and $V_{I N}-$ lines as well as the SDA and SCL line for the $\mathrm{I}^{2} \mathrm{C}$ communication (see Fig. 3.5). 


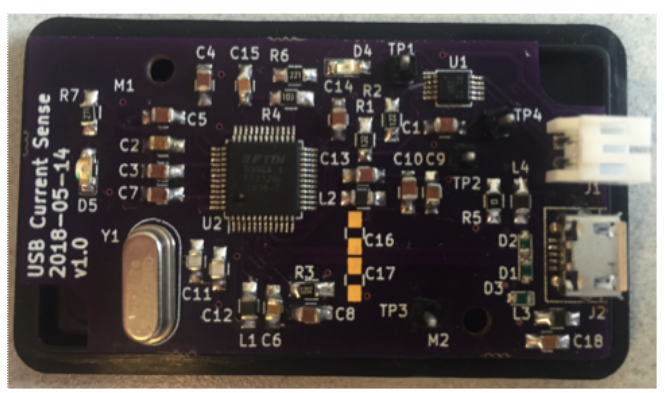

Figure 3.5 Custom PCB of the power measurement unit showing the INA226 sensor (U1), the FT232H Single-Channel USB to $\mathrm{I}^{2} \mathrm{C}(\mathrm{U} 2)$, the connector to the shunt resistance (J1) and the microUSB female connector (J2).

\subsection{Five-Axis Single-Wheel Test Bed}

Fig. 3.6a shows the five-axis single-wheel test bed used for this research. The position of the wheel unit along the $X^{S}$ and $Y^{S}$ axes and its orientation around the $Z^{S}$ axis can be controlled while the wheel unit is free to move along $Z^{S}$. The wheel axis is driven by a Maxon RE35 motor and MaxPos 50/5 driver. The load applied to the wheel was set to $1 / 4$ of the Husky weight by using a counterweight and the sinkage was measured using a linear string potentiometer. An ATI Delta IP60 six-axis force/torque sensor was also mounted between the wheel unit and the wheel itself.

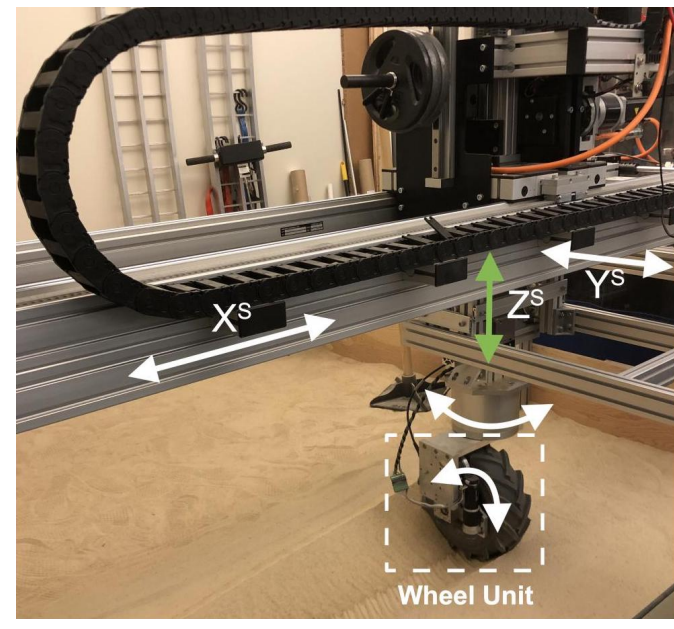

(a)

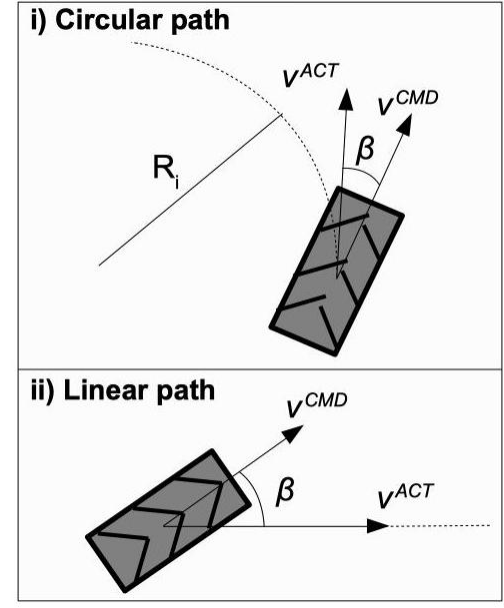

(b)

Figure 3.6 a) A Husky wheel placed in the single-wheel test bed. b) A Husky wheel performing i) a circular arc path of radius $R_{i}$ with a commanded linear velocity $v^{C M D}$, a tangential velocity $v^{A C T}$, and a slip angle $\beta$ and ii) a linear path . 
This setup was first used to reproduce the circular arc motion of an inner wheel during a set of turns of radii in and around the $B / 2<R<R^{\prime}$ range. Using the data collected during the Husky tests in the sandbox, the wheel axis was first rotated by $\beta$ before launching the test program. This program used the relevant $v^{C M D}$ and $v^{A C T}$ velocities to set the wheel angular velocity and the tangential velocity, respectively. Knowing the rover geometry, the inner wheel's motion radius $R_{i}$ was computed using the desired turning radius of the rover $(R)$ and the angular velocity around $Z^{S}$ was calculated knowing $v^{A C T}$ and $R_{i}$. The results of the tests listed above are presented in Section 5.3.2.

The wheel unit was also driven along the $X^{S}$ axis with various combinations of slip angle $\beta$, $v^{C M D}$ and $v^{A C T}$ velocities. These linear motions were used to validate the $2 \mathrm{D}$ slip-sinkage model presented in Section 5.3.1.

\subsection{Terrain}

The three testing sites (CSA Mars analogue terrain, WSNM and laboratory sandbox) were composed of different types of sand. In the laboratory sandbox, the Glenn Research Center-1 (GRC-1) simulant was used to replicate lunar and martian soil. This simulant was developed by Oravec et al. [29] to evaluate the tractive performance of lunar vehicles on a granular, dry cohensionless soil. To limit the soil disturbance to improve repeatability, the soil was prepared by loosening it with shovels, compacting it with a tamper and then leveling it with rakes. This procedure is thoroughly explained in [30] and was also used in [31] for drawbar pull tests in three different soil condition types (very loose, loose and medium dense). These soil conditions are defined as a range of relative density $D_{R}$ going from very loose to very dense and are shown in Fig. 3.8a. To measure this relative density for a particular soil, the first step is to gather a set of cone penetrometer tests. For each test (or insertion), the cone penetrometer measures the cone index (CI) as a function of depth, i.e. the pressure applied by the soil to the cone as it makes its way into the soil. The cone index is therefore a measure of the penetration resistance and an indication of soil strength. For the set of $90^{\circ}$ turns with varying turning radius in the controlled laboratory sandbox, 3 to 4 insertions were taken after the soil preparation and prior to the rover turn. Fig. 3.7 shows the average CI (of all the insertions prior to a turn) as a function depth. It first can be observed that the density distribution of the soil is consistent for all the tests. The low variability in the cone penetrometer insertions before each skid-steer rover turn confirms that the soil preparation was consistent, thus limiting the fluctuation in the soil properties and the variability in the power consumption. An important notion to consider is that the relationship between cone index and depth is linear for a uniform soil. The first $90 \mathrm{~mm}$ of sand can thus be assumed as approximately homogeneous and uniformly distributed. 


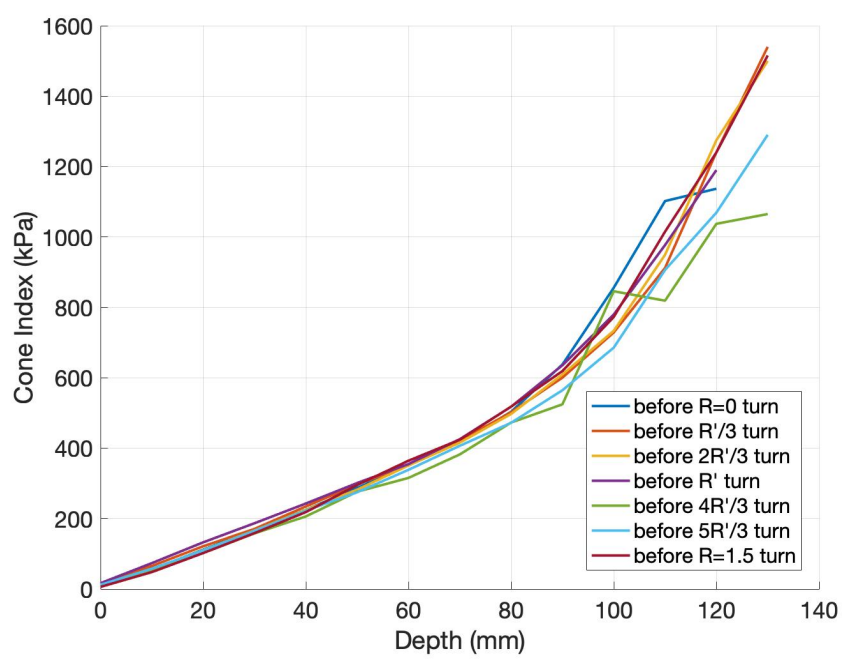

Figure 3.7 Cone Index (CI) as a function of depth for the fourth run of sandbox turns

From the cone index data, the cone index gradient $G_{C I}$, a measure of density and consistency of the soil, is computed following:

$$
G_{C I}=\frac{\sum_{i=1}^{n}\left(d_{i}-\bar{d}\right)\left(C I_{i}-\overline{C I}\right)}{\sum_{i=1}^{n}\left(d_{i}-\bar{d}\right)^{2}},
$$

where $n$ is the number of measurements in each insertions, $i$ is the insertion number, $d_{i}$ and $C I_{i}$ are the depth and cone index of insertion $i$ respectively, $\bar{d}$ and $\overline{C I}$ are the mean of all the depth and cone index values measured in the insertion, respectively.

Oravec et al. [29] correlated their cone index gradient measurements to a set of relative densities. This relative density, $D_{R}$, was determined after finding the minimum and maximum bulk density of the GRC-1 simulant. The minimum density, obtained by simply filling a container with a hopper, was found to be $1.60 \mathrm{~g} / \mathrm{cm}^{3}$ whereas the maximum density, obtained by vibrating the soil and applying a surface load, was found to be $1.89 \mathrm{~g} / \mathrm{cm}^{3}$. These values were used to set the $0 \%$ and $100 \%$ relative density. The linear fit of these scattered points is

$$
G_{C I}=0.0834 D_{R}+1.5811
$$

Using this equation, the relative density $D_{R}$ is calculated for all the cone penetrometer insertions in the controlled laboratory sandbox. As the cone index over depth presented in Fig. 3.7 seems to combine two approximately linear curves with two different slope values (before and after around $90 \mathrm{~mm}$ ), the relative density calculation was repeated as a function of increasing maximum depth. 
Fig. 3.8b shows the result of relative density as the range of depth increases. It can be concluded that the first $100 \mathrm{~mm}$ of the soil is medium dense and the deeper soil is in the dense to very dense range.

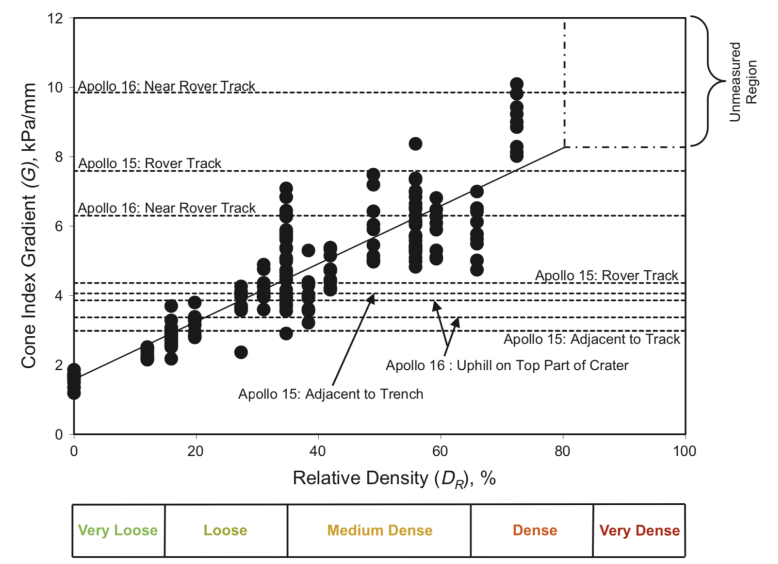

(a)

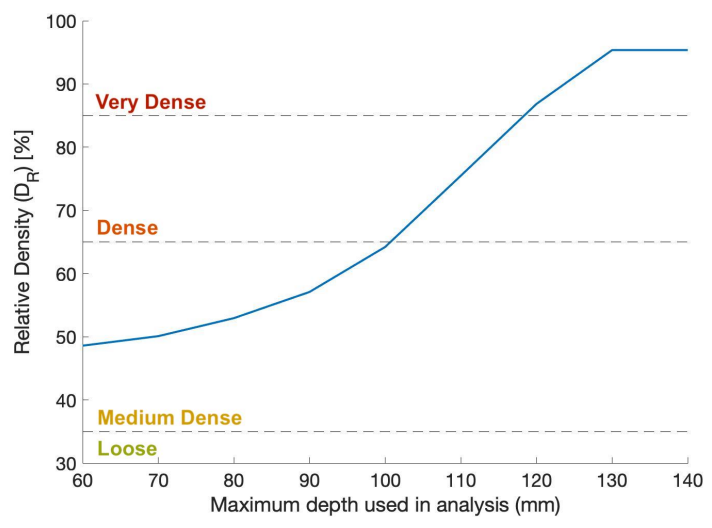

(b)

Figure 3.8 a) GRC-1 cone index gradient as a function of relative density $D_{R}$ of the tests performed by Oravec et al. [29] showing the fitted curve obtained (Eq. 3.2) and the soil condition types. b) Relative density $D_{R}$ obtained by averaging all the cone index gradient $G_{C I}$ from each CI vs. depth line presented in Fig. 3.7 with varying range of depth in the analysis.

Knowing that the Husky's ground clearance in $130 \mathrm{~mm}$ and that the rover rarely sinks to this point, the first $100 \mathrm{~mm}$ of soil are more critical to the analysis. Therefore, the condition of the GRC-1 simulant in the controlled laboratory sandbox after soil preparation is estimated at the limit between medium dense and dense soil. This results in a relative density of $65 \%$ and a bulk density of $1.7885 \mathrm{~g} / \mathrm{cm}^{3}$. Using other measurements from Oravec et al. paper, Table 3.3 shows the parameters of soil at the relevant condition used in this work. These parameters will be used when modeling the wheel sinkage and the sand bulldozing force in Section 5.3.1 and 5.4, respectively.

Table 3.3 GRC-1 Simulant Parameters Used in this Work

\begin{tabular}{ll}
\hline Parameter & Value \\
\hline Internal friction angle $\left(\phi_{i}\right)$ & $42^{\circ}$ \\
External friction angle $(\delta)$ & $0^{\circ}$ \\
Dry bulk density $(\rho)$ & $1.7885 \mathrm{~g} / \mathrm{cm}^{3}$ \\
Repose angle $\left(\varphi_{r}\right)$ & $42^{\circ}$ \\
Cohesion $(c)$ & $0 \mathrm{kPa}$ \\
\hline
\end{tabular}


The sands at the Canadian Space Agency and in White Sands are less characterized but cone penetrometer readings were taken at the Mars analogue terrain to compare the soil density with the GRC-1 simulant. Fig. 3.9 depicts the cone index as a function of depth for two test campaigns at the CSA Mars analogue terrain and the GRC-1 simulant in the laboratory sandbox. The CSA soil is outdoors and has a naturally higher moisture content which increases soil strength. It should also be noticed that the variability in the CSA cone penetrometer tests is significantly higher than the tests in the laboratory sandbox. This is expected due to the nature of an outdoor setting and as no extensive efforts were made to loosen, tamper and level the soil at CSA like it was done in the controlled laboratory sandbox. On the other hand, the average cone index for each depth was similar between the two CSA test campaign, in October 2018 and June 2019, despite varying weather. The slightly higher cone index results in October could be caused by the fact that it was raining and the soil was more damp.

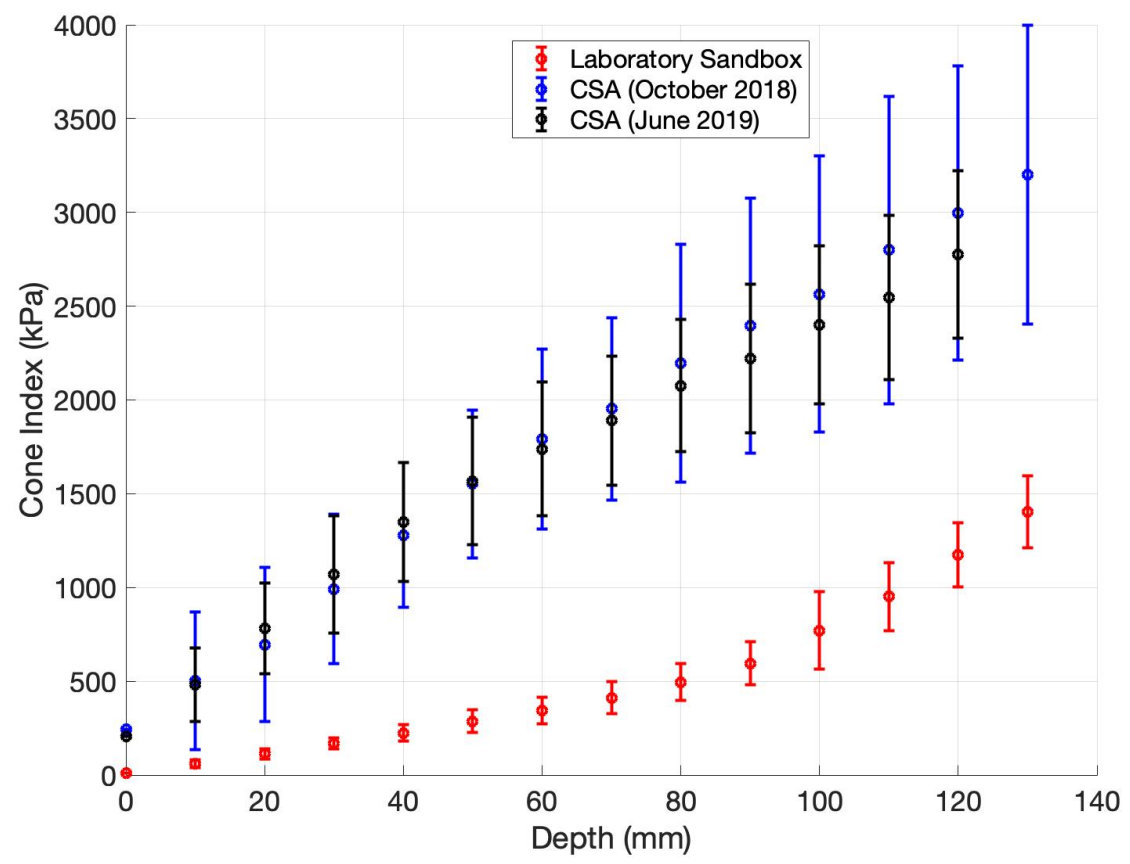

Figure 3.9 Cone index measurement comparison between the GRC-1 simulant and the sand at the CSA Mars analogue terrain. The scatter points represent the cone index average of all the insertion for each depth and the error bar represent one standard deviation above and under the average. 
The average of all the cone penetrometer tests performed at the two test sites also demonstrate that the density of sand at CSA is more than two times the density of the GRC- 1 simulant in the controlled laboratory sandbox. This can be observed in Fig. 3.9 but also by computing the cone index gradient $G_{C I}$. Table 3.4 summarizes the cone index gradients of the three test campaigns.

Table 3.4 Comparison of the cone index gradients obtained in the three test campaigns

\begin{tabular}{ll}
\hline Test Campaign & Cone Index Gradient $\left(G_{C I}\right)$ \\
\hline Laboratory Sandbox & $9.5 \pm 0.8 \mathrm{kPa} / \mathrm{mm}$ \\
CSA (October 2018) & $22.9 \pm 1.8 \mathrm{kPa} / \mathrm{mm}$ \\
CSA (June 2019) & $20.4 \pm 1.8 \mathrm{kPa} / \mathrm{mm}$ \\
\hline
\end{tabular}




\section{Chapter 4}

\section{Power Model Validation and Limitations}

This chapter presents an experimental investigation of the simplified ICR-based power model taken from the literature (Eq. 2.12). First, the Argo J5 and Clearpath Husky are driven in two different sloped sandy terrains (at the CSA Mars analogue terrain and White Sands National Monument, respectively) to validate that the roll angle of the rover can be neglected when operating on sandy slopes but also to show that the general format of Eq. 2.12 is acceptable for turns with radius $R>R^{\prime}$. Then, the Clearpath Husky is driven on hard flat terrain and the results are compared to the power prediction shown in Fig. 2.3, validating the model's key features.

On the other hand, limitations of the power model, which is typically used on hard terrain, are exposed using power measurements collected during an extensive test campaign in the laboratory sandbox with the Clearpath Husky platform. This motivates the need to model the effect of the soil that affects the power consumption of a skid-steer rover.

\subsection{Robot's Pitch and Roll Impact on Power Consumption}

The skid-steer rover is now considered to be operating on sandy slopes, as highlighted in the block diagram of Fig. 4.1 and the power model defined by Eq. 2.12 is investigated here. This section focuses of the third term of the power model to validate the linear relationship between the power and the vehicle's pitch as well as the exclusion of the vehicle's roll in the power model. 


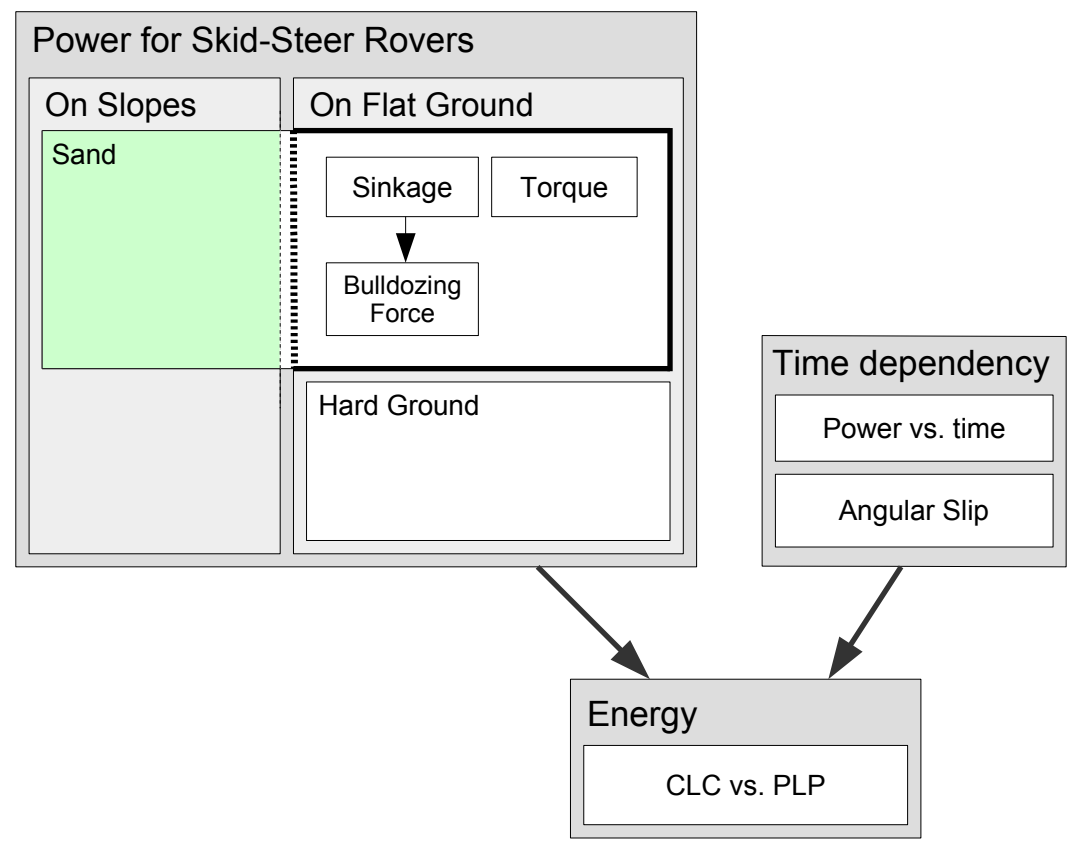

Figure 4.1 High level overview of the topics studied in this research, showing the context in which the pitch and roll impact on power consumption is studied.

Previous studies have used the slope's pitch $\theta_{s}$ and the vehicle's angle of attack $\gamma$ (i.e. the angle between a line along the gradient of the slope and the vehicle's heading) to describe skid-steered rover orientation on a slope. It is intuitive to use these parameters to study the traversability of a slope [16][32][33]. However, for power modeling, it is more insightful to see the impact of the vehicle's pitch $\theta$ and roll angles $\phi$ on the required power to perform a maneuver, as it was done in [17] for slip prediction purposes. This is because these are parameters that can be measured by the rover without explicit knowledge of the terrain geometry.

At the Mars analogue terrain, the Argo J5 performed a series of $0 \mathrm{~m}, 1 \mathrm{~m}, 2 \mathrm{~m}$ and $4 \mathrm{~m}$ turns and some straight line segments going up, down and across two different slopes at a constant $v_{c}=0.3$ $\mathrm{m} / \mathrm{s}$. The measured power results as a function of pitch and absolute roll value (measured by the IMU) were divided by maneuver and fitted against the following equation using the MATLAB Least-Squared Method fit function:

$$
P(\theta, \phi)=\xi \theta+\eta \phi+\zeta
$$

where $\xi, \eta$ and $\zeta$ are fit coefficients. The result of this power fitting as well as the raw power measurement data for the case of a $2 \mathrm{~m}$ turn are depicted in Fig. 4.2. Note that the cloud of power measurements doesn't cover a uniform distribution of pitch and roll values (instead, they follow the curve of the arc driven). This may influence the goodness of the fit. 


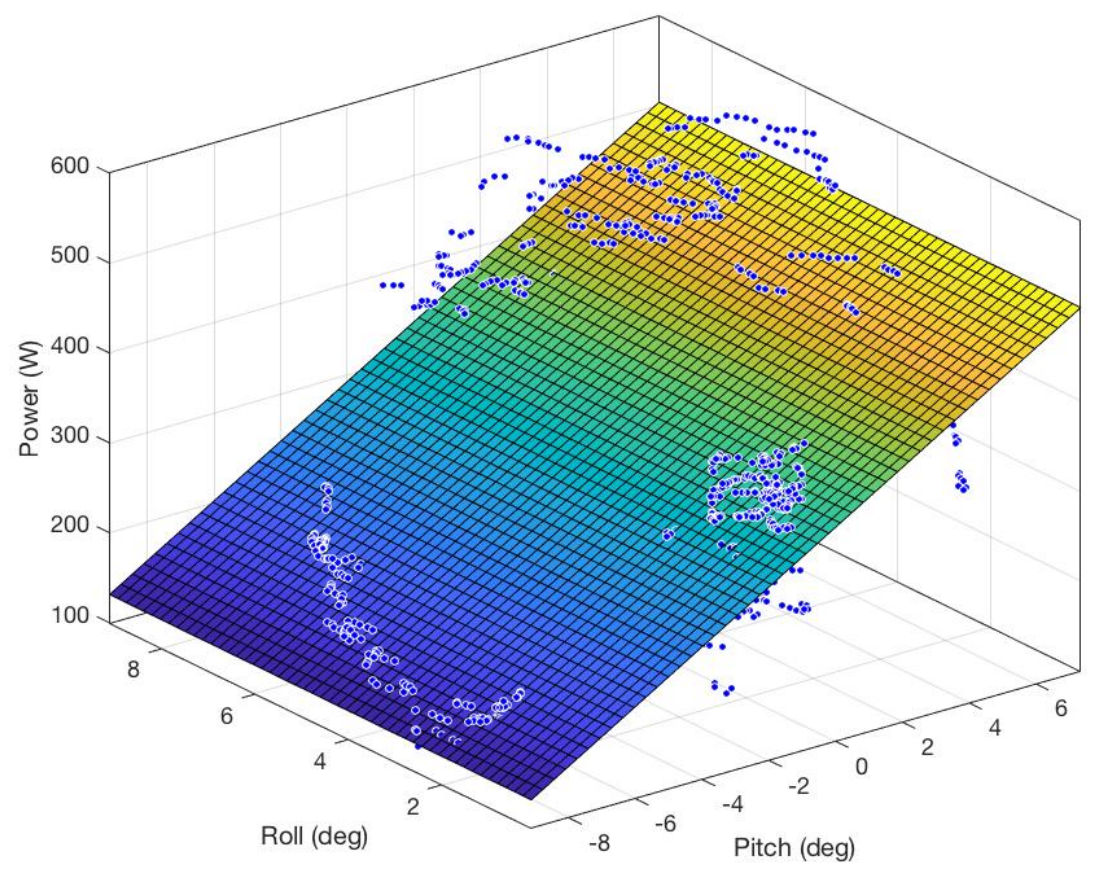

Figure 4.2 Linear regression of the fit against $P=\xi \theta+\eta \phi+\zeta$ for the case of $2 \mathrm{~m}$ turns

Table 4.1 illustrates the resulting coefficients and Table 4.2 includes the $95 \%$ confidence bound range of the coefficients as well as the $r^{2}$ coefficient of determination for all the maneuvers tested. It can easily be noticed that, if the point turn $(R=0 \mathrm{~m})$ is treated as a special case, the coefficient for power with respect to pitch is similar within a narrow range: $22.54 \leq \xi \leq 26.97$. It is also noted that the sensitivity to roll is very small and can be neglected for the purpose of modeling the power consumption in a sloped environment; the $95 \%$ confidence intervals in Table 4.2 for $\eta$ are not consistently above nor below zero, and sometimes straddle zero. As it can also be seen in Table 4.2, the coefficient of determination of the point turn test is low. This can be explained by the fact that the power consumed during point turn is highly variable but not consistently influenced by the robot's pitch and roll. Hence, we can consider the point turn as a special case that should be treated carefully when modeling the power consumption on slopes. The $r^{2}$ coefficient for the other maneuvers are reasonable considering that the power required to maneuver a skid-steered wheeled robot is not exclusively dependant on the orientation of the robot. 
Table 4.1 Fitted Linear Equations

\begin{tabular}{cl}
\hline Turning Radius & Resulting Linear Regression Equation \\
\hline 0 & $\mathrm{P}(\theta, \phi)=1.899 \theta-3.719 \phi+1108$ \\
1 & $\mathrm{P}(\theta, \phi)=26.36 \theta-3.571 \phi+565.4$ \\
2 & $\mathrm{P}(\theta, \phi)=22.54 \theta-0.07985 \phi+338.1$ \\
4 & $\mathrm{P}(\theta, \phi)=24.21 \theta-0.2165 \phi+222.4$ \\
$\infty$ & $\mathrm{P}(\theta, \phi)=26.97 \theta+2.564 \phi 160.7$ \\
\hline
\end{tabular}

Table 4.2 95\% Confidence Bounds of the Power Coefficients

\begin{tabular}{cccc|c}
\hline $\begin{array}{c}\text { Turning } \\
\text { Radius }\end{array}$ & $\xi$ & $\eta$ & $\zeta$ & $r^{2}$ \\
\hline 0 & {$[1.098,2.701]$} & {$[-4.862,-2.577]$} & {$[1101,1115]$} & 0.0804 \\
1 & {$[25.5,27.53]$} & {$[-5.165,-1.977]$} & {$[558.2,572.5]$} & 0.7466 \\
2 & {$[21.69,23.38]$} & {$[-1.26,1.1]$} & {$[331.7,344.5]$} & 0.7069 \\
4 & {$[23.12,25.3]$} & {$[-1.455,1.022]$} & {$[214.8,230.1]$} & 0.8335 \\
$\infty$ & {$[26.52,27.42]$} & {$[1.999,3.129]$} & {$[157.5,163.8]$} & 0.9106 \\
\hline
\end{tabular}


Fig. 4.3 clearly illustrates the consistent coefficient (i.e. slope) for power with respect to pitch, regardless of the non-zero turning radius.

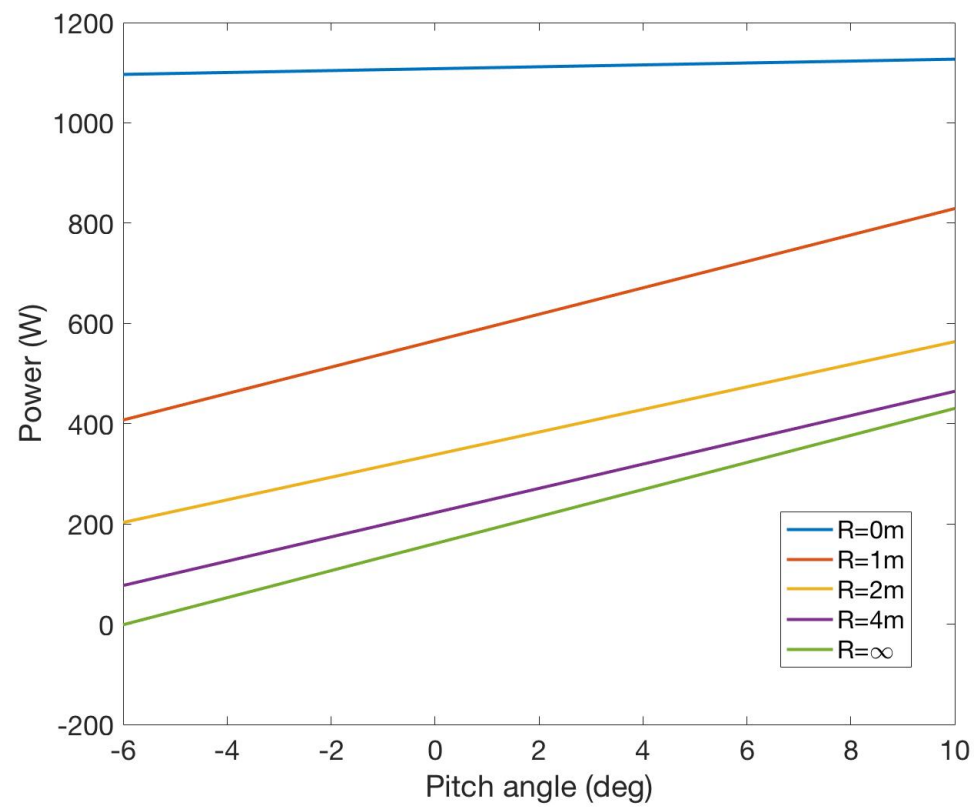

Figure 4.3 Power vs. pitch of the resulting linear equations when $\eta=0$. Slopes of the equations are consistent for $R \geq 1 \mathrm{~m}$.

From the results demonstrated in this section, it can be confirmed that it is valid to neglect the effect of roll in the power model, as given by Eq. 2.12. It can also be concluded that a linear relation between pitch and power consumed is reasonable for vehicle pitch angles within $\pm 15^{\circ}$, consistent with the $\sin \theta \approx \theta$ assumption.

\subsection{Validation of the Power Model on Slopes}

Using the conclusion drawn in the first testing phase, the power model defined in Eq. 2.12 was further tested by relating the power consumed by the Husky's DC motor with the instantaneous turning radius $(R)$ and vehicle's pitch $(\theta)$ when driving in the dunes of the White Sands National Monument, at a constant $v_{c}=0.3 \mathrm{~m} / \mathrm{s}$. The instantaneous turning radius at each time stamp is computed using Eq. 2.4 with $B_{s}=1.1 \mathrm{~m}$ (the defined slip track of the Husky A200). For computational reasons, turning radii larger than $20 \mathrm{~m}$ were neglected and a $0 \mathrm{~m}$ turning radius (i.e. point turn) was set to $0.001 \mathrm{~m}$. The $c_{1}, c_{2}$ and $c_{3}$ coefficient were empirically computed using the MATLAB fit function against the $R \geq R^{\prime}$ case of Eq. 2.12. It should be noted that a linear fit is computed against $1 / R$ and $\theta$ as input variables. 
The resulting curve is depicted in Fig. 4.4 and the coefficients are shown in Table 4.3. The coefficient of determination for this least-squared linear fit is $r^{2}=0.685$.

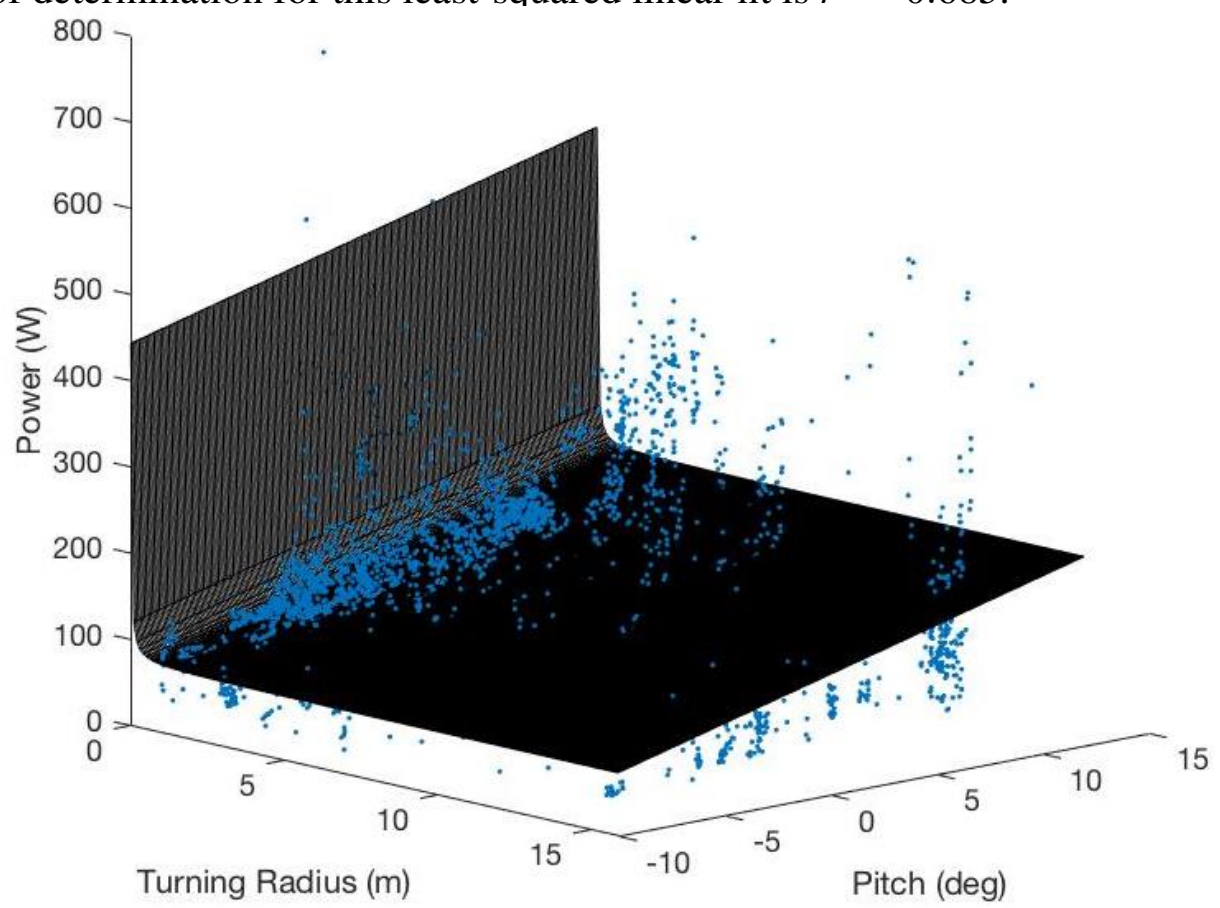

Figure 4.4 Fitted power measurements obtained in White Sands National Monument against $P=$ $\frac{c_{1}}{R}+c_{2}+c_{3} \theta$, where $P$ is the power required to perform a turn of radius $R$ with a robot's pitch of $\theta$ degrees.

Table 4.3 Power fit results of the $P=c_{1} / R+c_{2}+c_{3} \theta$ for the random drives at the White Sands National Monument

\begin{tabular}{ccc}
\hline Power Coefficient & Value & 95\% Confidence Bounds \\
\hline$c_{1}$ & 5.55 & {$[5.269,5.831]$} \\
$c_{2}$ & 139.4 & {$[138.1,140.7]$} \\
$c_{3}$ & 6.608 & {$[6.418,6.799]$} \\
\hline
\end{tabular}

From these results, it can be concluded that the trends of simplified power model are valid to some extent $\left(r^{2}=0.685\right.$ ) when manoeuvring a skid-steered wheeled robot in a sloped environment, on sand. However, the results clearly show that additional work should be done to improve the power modeling on uneven loose soil, in order to increase the coefficient of determination for the measured power curve fitting. One possible future extension is to relax the assumption of symmetric ICR. 


\subsection{Power Model Validation on Hard Terrain}

In this section, the power consumption of skid-steer rovers is studied on hard flat terrain, as shown in Fig. 4.5. As the ICR-based power model derived in Section 2.2 is typically for hard surfaces, the first part of the validation is to perform a set of $90^{\circ}$ turns with the Clearpath Husky on a flat concrete surface. This investigation goes further than previous work in the literature, by including a focus on turns at $R^{\prime}$ and in the special case of turning radii identified in Section 2.1.2 $\left(B / 2<R<R^{\prime}\right)$.

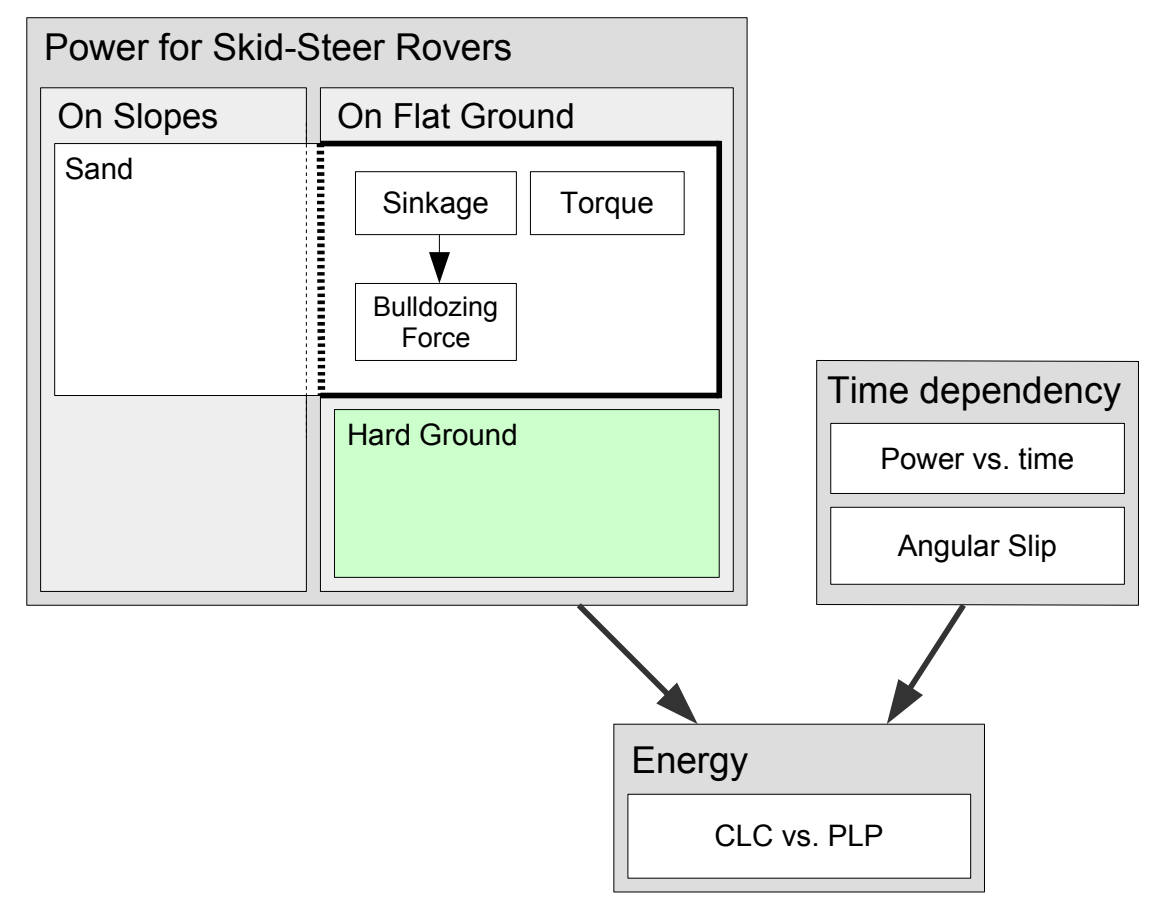

Figure 4.5 High level overview of the topics studied in this research, showing the context in which the power model is first validated.

The instantaneous power consumed during each turn is first plotted as a function of time in Fig. 4.6a. As shown in Eq. 2.8 in Section 2.1.1, the $v_{c}$ velocity constraint imposed corresponds to a constant commanded angular velocity for $0 \leq R \leq R^{\prime}$. Fig. 4.6a shows a nearly constant time required to complete the $90^{\circ}$ turns for $0 \leq R \leq R^{\prime}$. As expected, the power remains approximately constant throughout each turn; the small variations are due to noise in the measurements. The average power consumed during each $90^{\circ}$ turn is also computed and the data points are plotted for the three runs of test in Fig. 4.6b. The average line can be compared to the power model prediction, under the constant $v_{c}$ constraint, depicted in Fig. 2.3. As the model predicts, the measured average power is approximately constant from turn ranging from a point turn $(R=0)$ to $2 R^{\prime} / 3$. However, the experimental data shows that the average power during the turn drops prior to $R^{\prime}$. 


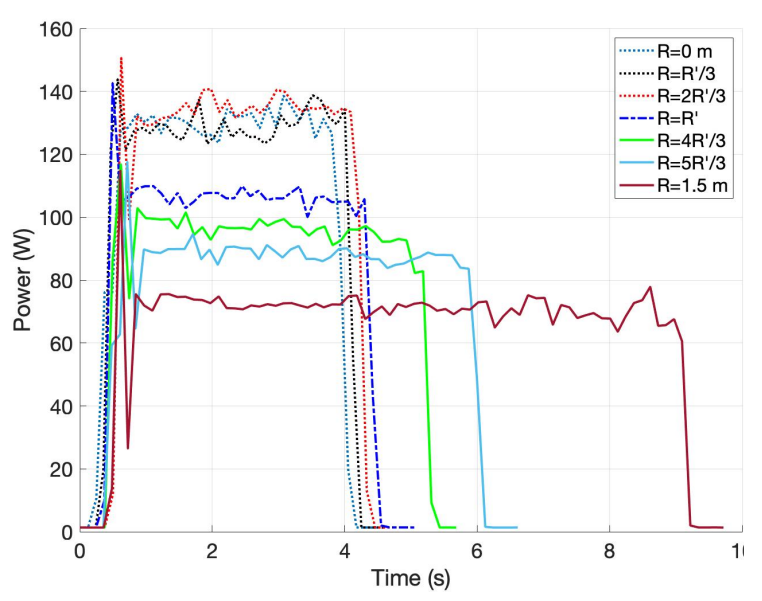

(a)

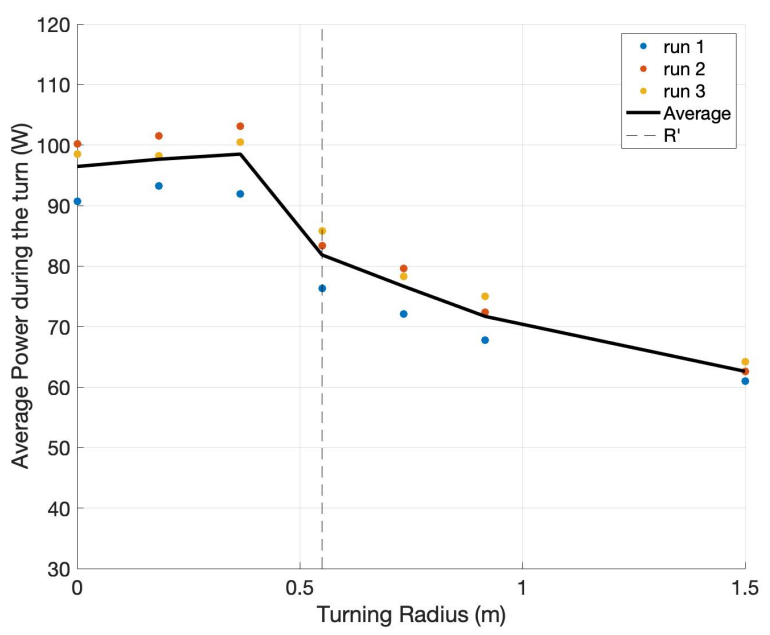

(b)

Figure 4.6 a) Power consumption with respect to time as each turn progresses. It can be observed that the power remains relatively constant as small variations are cause by noise in the measurements. b) Average power consumption during a set of $90^{\circ}$ turns, on hard concrete ground. As predicted in Fig. 2.3, the power is constant as $R$ increases from 0 to $2 R^{\prime} / 3$ but the power drops from the $2 R^{\prime} / 3$ to the $R^{\prime}$ turn.

Even though the power isn't constant up to and including $R^{\prime}$ itself, the overall trend of Fig 2.3 is reproduced experimentally in Fig. 4.6b. Therefore, this suggests that the power model can provide a valid estimation of the power consumption of a skid-steer rover on a flat hard surface such as concrete. It will be shown in Section 6.1 that the optimal radius for a CLC path is found to be at $R^{\prime}$, both experimentally and theoretically with the existing power model. This is consistent with the conclusion that the power consumption of a skid-steer rover is properly modeled using the existing simplified model, when operating on hard terrain. 


\subsection{Limitation of the Existing Model}

The Clearpath Husky A200 is now driven on flat sand to observe its power consumption but also the time dependency of the power. Under these conditions, highlighted in the block diagram of Fig. 4.7, a clear limitation of the power model examined in this work is uncovered. The power consumption over time and the average power during a set of turns are thoroughly analyzed in this section.

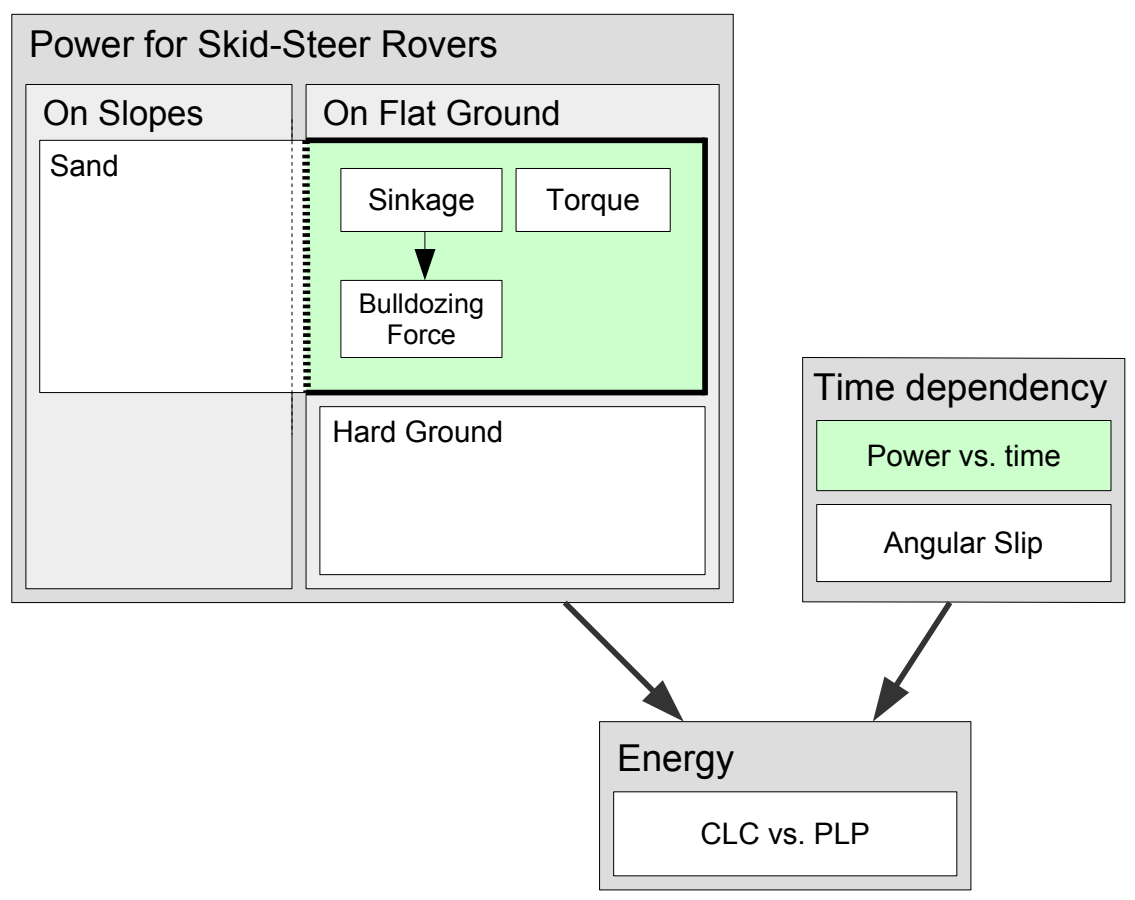

Figure 4.7 High level overview of the topics studied in this research, showing the context in which the limitations of the power model are observed.

\subsubsection{Power Consumption over Time}

Fig. 4.8 depicts the power consumption with respect to time for the set of turns performed in the GRC-1 soil bed. From this figure, it should be noted that the total power consumption of the left and right motor drivers is increasing with time in the case of maneuvers with $R \leq R^{\prime}$. This is a clear difference with the experimental power measurements on hard ground presented in Fig. 4.6a. It should also be noted that the magnitude of the power consumed on sand reach around twice the power consumed on hard ground for turns with $0 \leq R \leq R^{\prime}$. This gap decreases as $R$ increases above $R^{\prime}$; the effects of loose soil on power consumption are less significant for wider arcs, when less lateral skidding is required to achieve the arc path. 
Another important observation is that the time to complete the $90^{\circ}$ rotation increases as $R$ approaches $R^{\prime}$, which wasn't the case for the turn on hard terrain. This is most likely due to the fact that the rover needs to overcome a higher resistance force, in particular when the inner wheels are fixed and not excavating the soil (at $R^{\prime}$ ). This will be discussed in detail in Section 6.2.

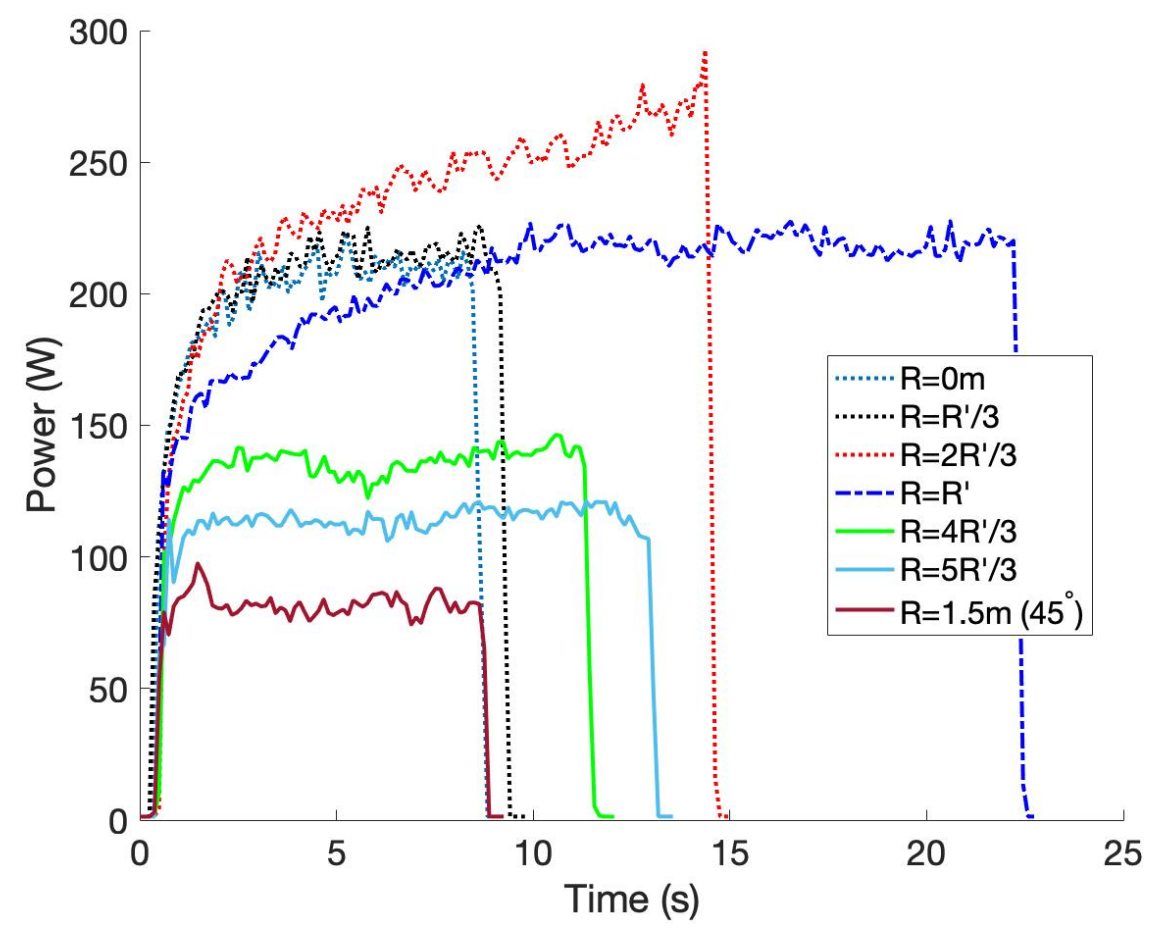

Figure 4.8 Power consumed over time for a set of turns performed in the controlled laboratory sandbox. For $R \leq R^{\prime}$ (dotted lines), power rises as the turn progresses. 


\subsubsection{Average Power Consumption during a Turn}

Experimental data demonstrates that the power consumption of a skid-steered wheeled robot is not constant below $R^{\prime}$. In order to demonstrate this, $90^{\circ}$ turns of radii around $R^{\prime}=0.55 \mathrm{~m}$ are executed with the Clearpath Husky A200 platform in the controlled laboratory sandbox. Three different $v_{c}$ constant values $(0.15 \mathrm{~m} / \mathrm{s}, 0.3 \mathrm{~m} / \mathrm{s}$, and $0.45 \mathrm{~m} / \mathrm{s})$ are tested and the average power measured during these maneuvers is depicted in Fig. 4.9. The $v_{c}=0.45 \mathrm{~m} / \mathrm{s}$ set was only tested once as the rover got stuck during the $R^{\prime}$ turn; the outer wheels sank too much as they were rotating at a high linear velocity $\left(v_{o}^{C M D}=0.9 \mathrm{~m} / \mathrm{s}\right)$.

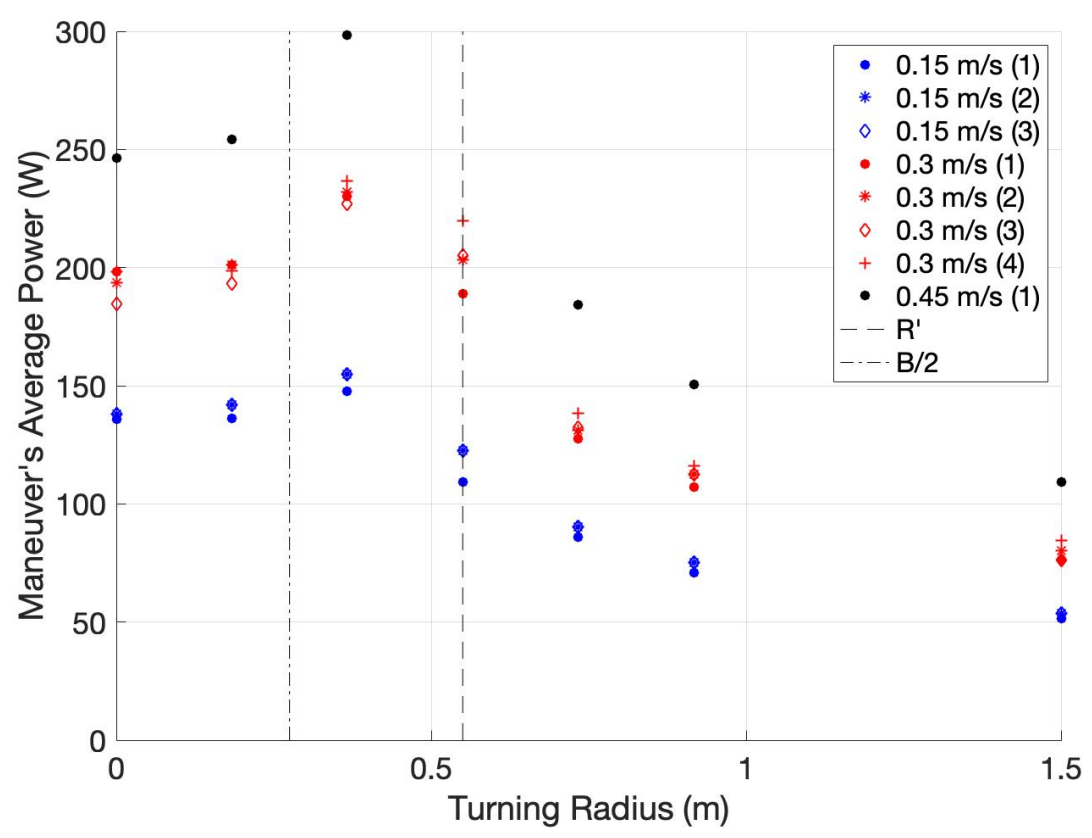

Figure 4.9 Average power consumed during a set of $90^{\circ}$ turns performed in the laboratory's sandbox, with three $v_{c}$ values. Non-constant power is observed for all cases in the $0 \leq R \leq R^{\prime}$ range (contrast with Fig. 2.3).

It is clear that the power doesn't remain constant for turns where the inner wheels are rotating backwards as the power consumption rise significantly (to values 15\% to $20 \%$ higher than $P(0)$ ) and peak in the special case region region identified in Section 2.1.2, i.e. when the center of rotation lies between the inner wheels and their ICR $\left(B / 2<R<R^{\prime}\right)$. Fig. 4.9 shows that the power trend with respect to $R$ is not dependent on $v_{c}$ as the proportional rise in average power from the point turn to the peak is approximately constant for all $v_{c}$ values tested. 
Fig. 4.10 compares the results recorded at CSA with the results from the controlled sandbox tests. All these tests were performed with $v_{c}=0.3 \mathrm{~m} / \mathrm{s}$.

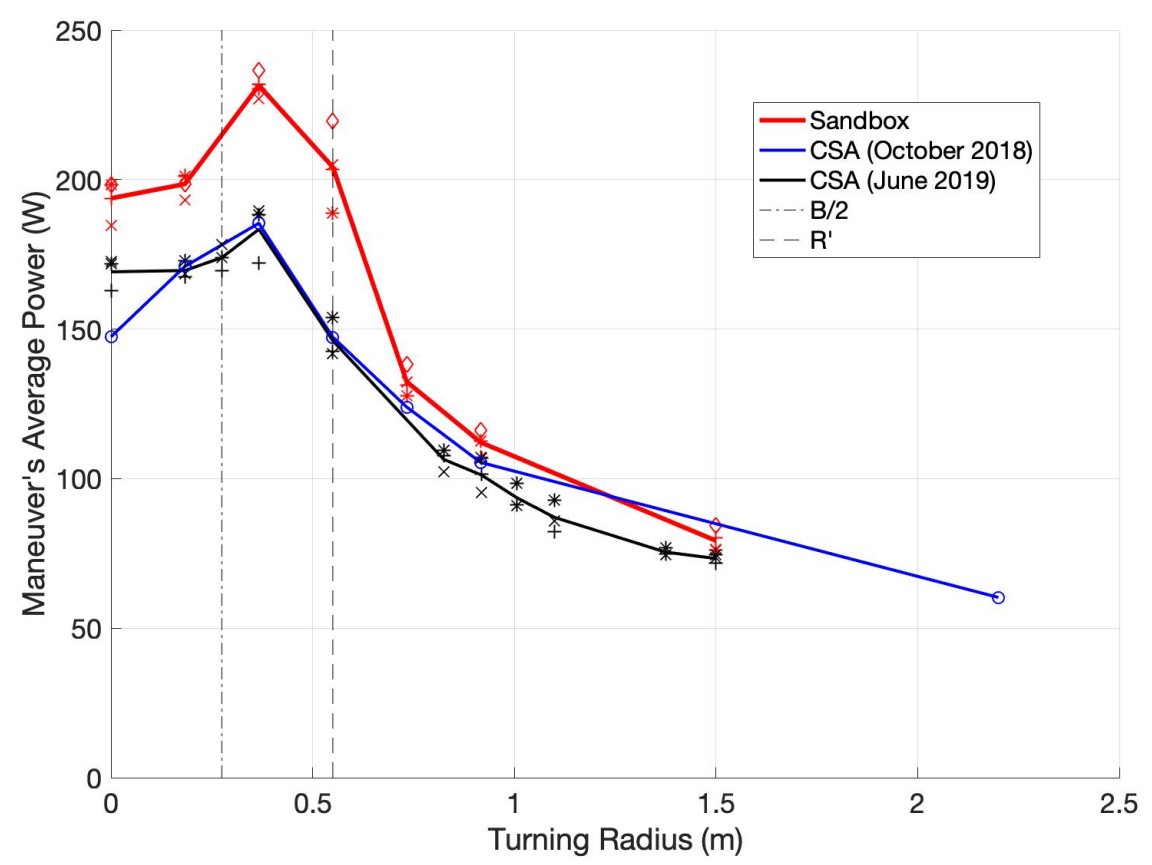

Figure 4.10 Average power consumed during a set of $90^{\circ}$ turns performed in the laboratory's sandbox (in red) and during the two test campaigns at the CSA Mars analogue terrain (in blue and black), with $v_{c}=0.3 \mathrm{~m} / \mathrm{s}$.

As the CSA Mars analogue terrain sand was measured to be firmer than the GRC-1 soil simulant of the sandbox, the effect of loose soil on power consumption in the latter case are more significant, hence requiring more power on average to achieve turns with $0 \leq R \leq R^{\prime}$. As $R$ increases, less skidding is required for the rover to turn and the average power consumption is approximately the same, regardless of the soil properties.

It was shown in Section 4.4.1 that the power rises as the turn progresses on loose soil, when $0 \leq R \leq R^{\prime}$. This means that only representing the average power consumed during a $90^{\circ}$ turn as a function of $R$ doesn't fully cover the instantaneous power consumption of a skid-steer rover as it did on hard terrain, where the power remained approximately constant throughout the turn (see Section 4.3). Accordingly, a more insightful representation would be to show the average and the maximum power, i.e. the power at the end of the $90^{\circ}$ rotation, recorded during the turn. Fig. 4.11 depicts the average (solid line) and maximum (dotted line) power measured during the set of turns in the laboratory sandbox and at the CSA Mars analogue terrain. As expected, the gap between the average and maximum power expands as $R$ increases from 0 to $2 R^{\prime} / 3$, the turn in the special 
case range, and then shrinks until the two lines almost touch each other. This result is similar to the observations made in Section 4.4.1, where the higher rise in power consumption as the turn progresses was observed at $R=2 R^{\prime} / 3$.
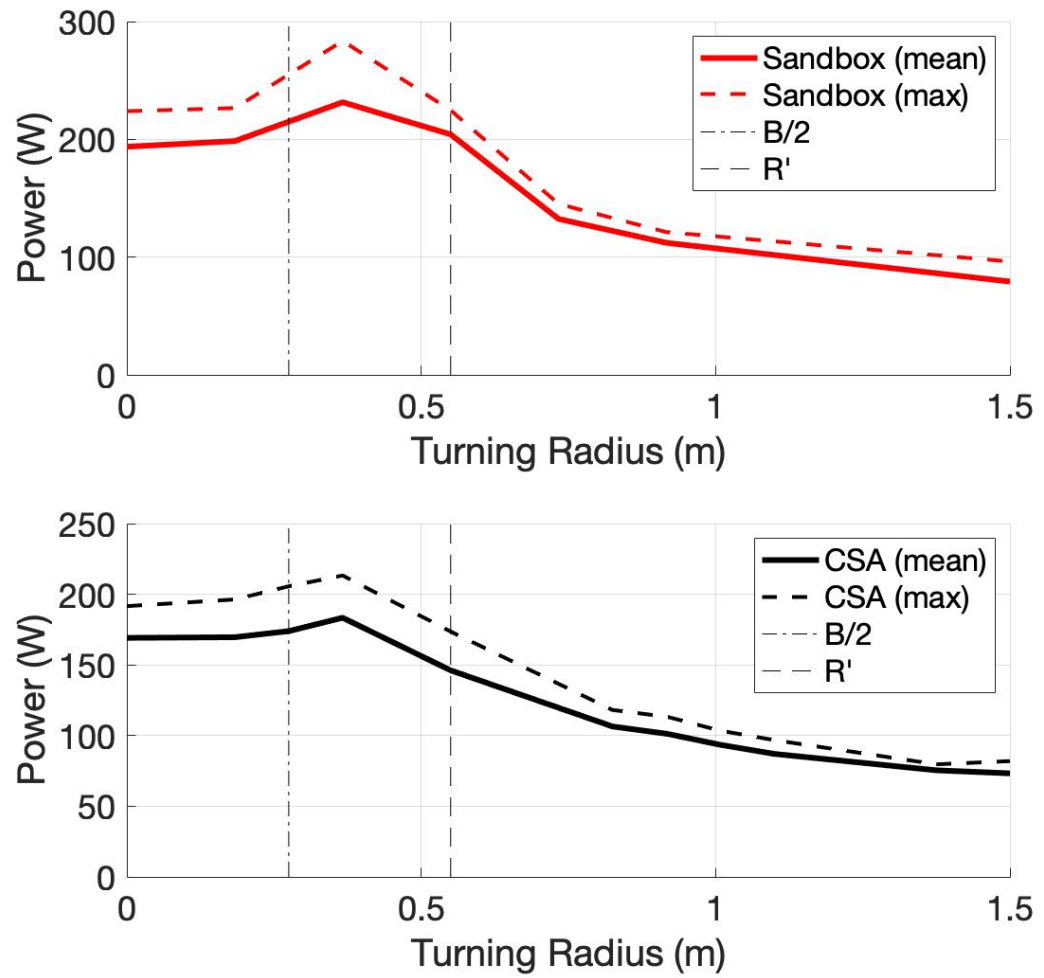

Figure 4.11 Average and maximum power consumed during for set of turn tested in the controlled laboratory sandbox (top) and at the CSA Mars analogue terrain (bottom).

It should be noted that similar results were observed when repeating this set of tests at the CSA Mars analogue terrain with another skid-steered wheeled robot, the Argo J5. However, the rise in power as the turn progresses (for turns with $0 \leq R \leq R^{\prime}$ ) as well as the peak in average power in the special case region, i.e. $B / 2<R<R^{\prime}$ were less significant. That being said, average power still peaked by close to $10 \%$ higher than $P(0)$ in the $0 \leq R \leq R^{\prime}$ range. 


\section{Chapter 5}

\section{Investigating the Contributing Factors to the Increasing Power Consumption on Sand}

Experimentally measured power consumption by the Husky on loose soil shows important discrepancies compared to the model for hard ground presented in Eq. 2.16, as discussed in the previous chapter. Further analysis of experimental data exposes two possible contributing factors causing the increase in power consumption below $R^{\prime}$ : wheel sinkage and motor torque. A particular focus is put on the inner wheels for the special case identified, i.e. when they are rotating backwards but being dragged forward by the outer wheels. To enable the requisite analyses, the slip angles and actual velocities of the inner (and outer) wheels are required. These are computed from experimental data in Section 5.1. Wheel torques are then related to these slip angles angle velocities in Section 5.2. Finally, Section 5.3 and 5.4 model the wheels' sinkage and sand bulldozing force, respectively, based on slip angles and wheel velocities.

As shown in the block diagram of Fig. 5.1, this chapter presents an investigation of the contributing factors affecting power consumption of skid-steer rovers operating on flat loose soil such as sand. 


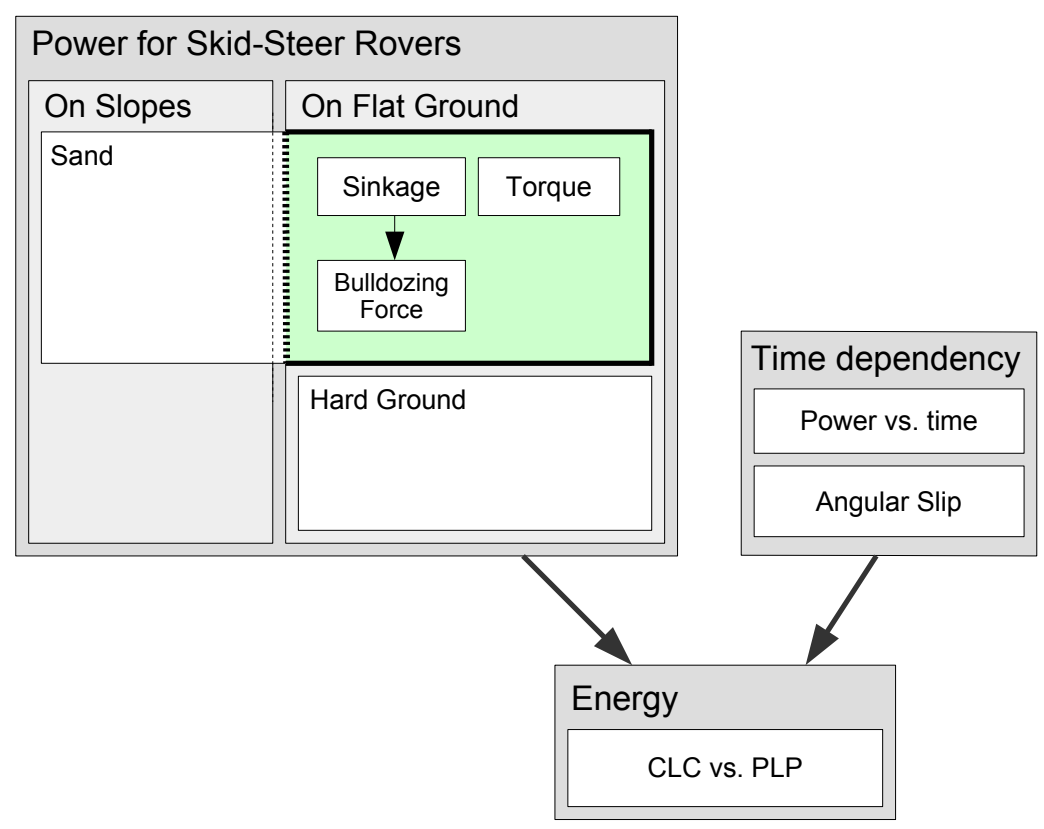

Figure 5.1 High level overview of the topics studied in this research, highlighting the conditions in which the contributing factor of increase power consumption are studied.

\subsection{Data Post-processing}

In this section, the data gathered during the Husky test campaign in the controlled laboratory sandbox, i.e. the set of turns presented in the previous chapter, is processed to investigate the contributing factor of increased power consumption on loose soil shown in the previous chapter. Once the data loss of total station measurements of the landmark prism on the Husky is corrected, the inner and outer wheels' slip angle $\left(\beta_{i}\right.$ and $\left.\beta_{o}\right)$ and actual velocity $\left(v_{i}^{A C T}\right.$ and $\left.v_{o}^{A C T}\right)$ can be estimated. These parameters are key for further interpretations and analyses.

\section{Total Station Data Loss Correction}

The total station used in this research records the position $\left({ }^{T S} x_{l p},{ }^{T S} y_{l p},{ }^{T S} z_{l p}\right.$ coordinates in the total station frame) of the landmark prism at a frequency of $5 \mathrm{~Hz}$. Since the total station doesn't timestamp its position measurement, the first step is to graphically determine the start and end points, i.e. when the distance between two data points is not caused by noise. Knowing the measuring frequency of the total station, the time of each measurements is estimated, starting at 0 with increments of 0.2 seconds.

During post-processing activities, it is noticed that some data points weren't captured during the tests, most likely due to the rover's vibration causing the laser pointer to lose track of the 
reflecting prism. To correct this issue, position measurements are added, following the trend of the curve. Accordingly, the distance between each point is computed to detect the outliers using an Hampel filter. The MATLAB function used defines an outlier as being a sample that is at least three standard deviations from the median of the window composed of the sample itself and three samples on each side. The function also returns this window median value for all the samples. This value is used to compute the distance over median ratio, $r_{d i s t}$, for each outlier detected. For example, one data point is added if $1.5<r_{\text {dist }} \leq 2.5$, two data points are added if $2.5<r_{\text {dist }} \leq 3.5$ and so on. The location of the added ${ }^{T S}\left[x_{l p}, y_{l p}\right]$ points are determined by performing a quadratic polynomial fit with the neighbor points. Fig 5.2 displays an example of the data loss correction for the case of a $R^{\prime}$ turn. The added landmark prism positions are displayed in pink.
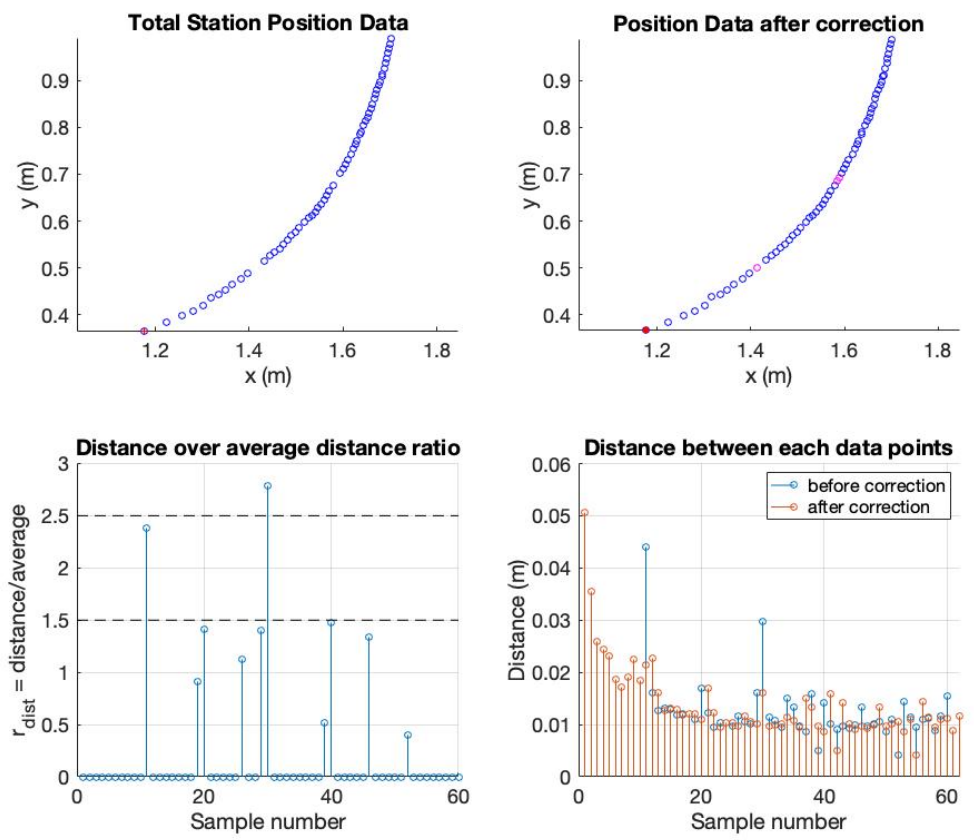

Figure 5.2 Total station data loss correction example for the case of a $R^{\prime}$ turn in the sandbox. The top left plot shows the total station measurements prior to the correction, the bottom left plot shows the $r_{\text {dist }}$ calculation for all the data points (or sample), the top right plot shows the position data with the added points (in pink), and finally the bottom right shows the distances between the point before and after the correction.

\section{Inner and Outer Wheels' Slip Angle ( $\beta_{i}$ and $\beta_{o}$ )}

For simplicity, the position of the rover's center of rotation, i.e. $I C R_{v}$ was assumed to be along a line perpendicular to the wheels, passing through the geometric center of the skid-steer rover. In other words, the small offset between the actual velocity of the rover $(\vec{v})$ and the vehicle's $Y$ - 
axis shown in Fig. 1.3 was neglected, i.e. $v_{x}=0$. As mentioned previously, this is a reasonable assumption for slow planetary rovers not experiencing dynamic lateral slippage.

The corrected position measurements of the landmark prism are then used to calculate the actual turning radius. The procedure to find this actual (or measured) turning radius is to first fit the set of $\left(x_{l p}, y_{l p}\right)$ data against the equation of a circle, i.e. $\left(x-x_{c}\right)^{2}+\left(y-y_{c}\right)^{2}=R_{l p}^{2}$, to extract the turning radius $R_{l p}$. Since all the turns performed in the experiments are left turns, the $\delta x_{l p}=21 \mathrm{~cm}$ offset (as shown in Fig. 5.3 between the center of the rover and the landmark prism is subtracted from the obtained $R_{l p}$ to get the measured (or actual) turning radius $R_{A C T}$.

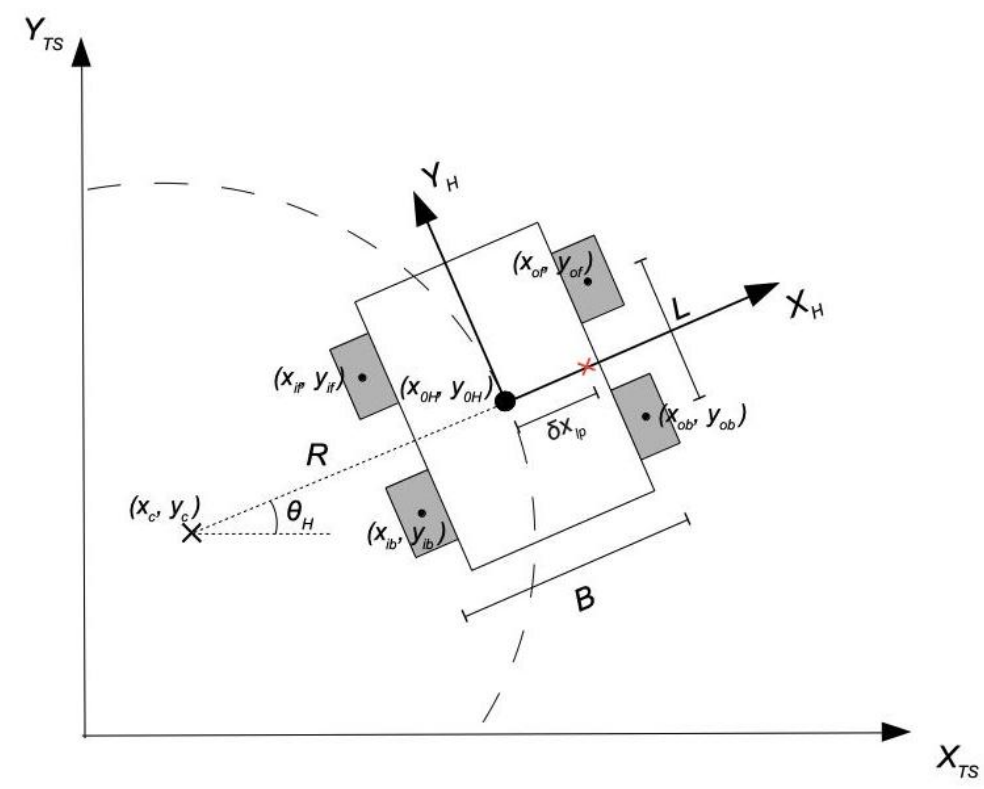

Figure 5.3 Position of the landmark prism (in red) and the four wheels in the Husky and total station coordinate frames

Using the geometry of the rover, the actual slip angles for the inner and outer wheels, $\beta_{i}$ and $\beta_{o}$ respectively, are found using Eq. 5.1 and 5.2. As described in Section 2.1.2, since the slip angle is defined as the angle between $v^{A C T}$ and $v^{C M D}$, the inner wheels' slip angle goes above $90^{\circ}$ in the special case set of turning radii, i.e. $B / 2<R_{A C T}<R_{A C T}^{\prime}$.

$$
\begin{gathered}
\beta_{i}=\left\{\begin{array}{c}
90^{\circ}+\tan ^{-1}\left(\frac{\left|2 R_{A C T}-B\right|}{L}\right), B / 2<R_{A C T}<R_{A C T}^{\prime} \\
90^{\circ}-\tan ^{-1}\left(\frac{\left|2 R_{A C T}-B\right|}{L}\right), \text { otherwise }
\end{array}\right. \\
\beta_{o}=90^{\circ}-\tan ^{-1}\left(\frac{2 R_{A C T}+B}{L}\right)
\end{gathered}
$$


Note that the otherwise case in Eq. 5.1 includes $R_{A C T}=R_{A C T}^{\prime}$ which, as described in Section 2.1.2, has $v^{C M D}=0$. The inner and outer wheels' slip angle obtained for three runs of turns is shown in Fig. 5.4 and compared to the expected slip angle $\left(\beta_{i}^{E X P}\right.$ and $\left.\beta_{o}^{E X P}\right)$ that would be obtained if $R_{A C T}=R_{C M D}$, where $R_{C M D}$ is the commanded turning radius.

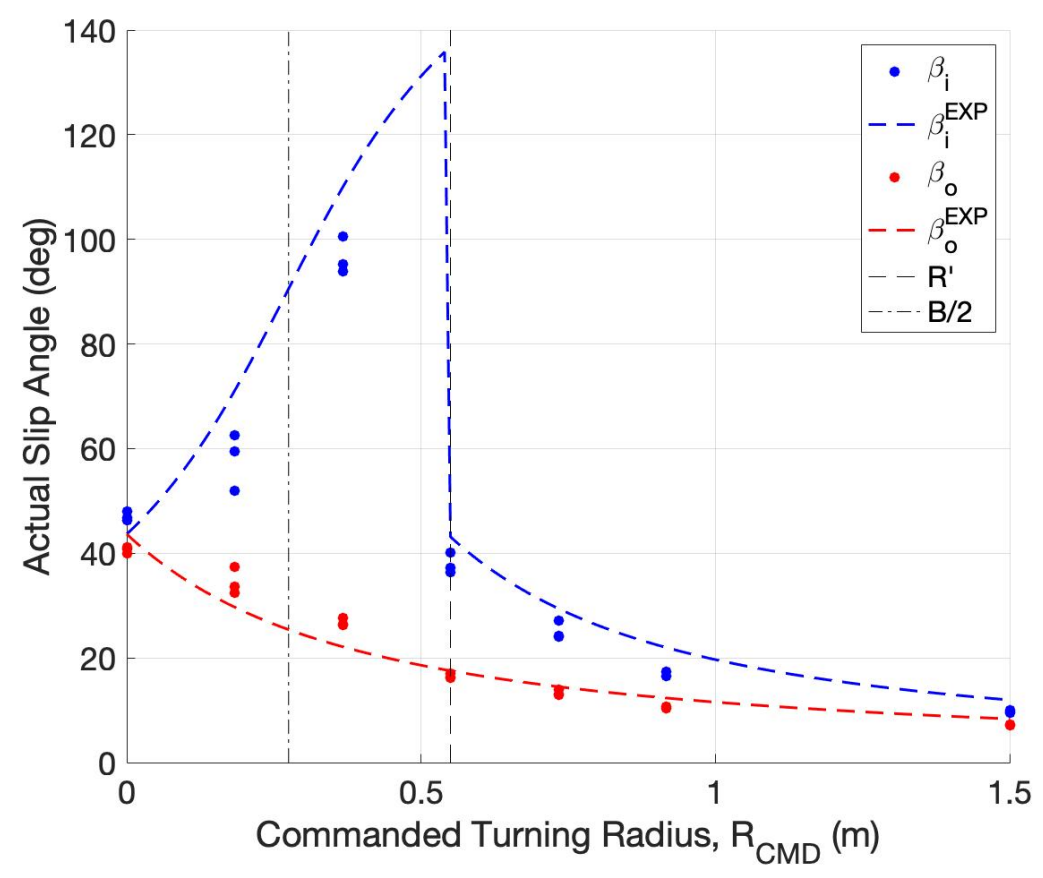

Figure 5.4 Resulting actual inner and outer wheel slip angle, $\beta_{i}$ and $\beta_{o}$ respectively, as a function of the commanded turning radius $R_{C M D}$ of three runs of $90^{\circ}$ turns with the Husky in the GRC-1 simulant of the laboratory sandbox.

\section{Inner and Outer Wheels' Actual Velocity $\left(v_{i}^{A C T}\right.$ and $v_{o}^{A C T}$ )}

Furthermore, using the position data and the IMU yaw $(\psi)$ angle measured, we can find the position of each wheel using the transformation matrix ${ }_{L P}^{T S} T$. This matrix allows us to get the inner front and back as well as the outer front and back wheel coordinates (with subscripts if, ib, of and $o b$ respectively) in the total station frame using the known geometry of the Husky. The ${ }_{L P}^{T S} T$ matrix is defined as

$$
{ }_{L P}^{T S} T=\left[\begin{array}{ccc}
\cos \theta_{H} & -\sin \theta_{H} & x_{l p} \\
\sin \theta_{H} & \cos \theta_{H} & y_{l p} \\
0 & 0 & 1
\end{array}\right]
$$

where $\theta_{H}$ is the angle offset from the total station frame to the Husky frame and $\left(x_{l p}, y_{l p}\right)$ are the 
coordinates of the landmark prism, in the total station frame. To find the angle offset between the two coordinates systems, we use the final position of the landmark prism $\left(x_{l p}^{f}, y_{l p}^{f}\right)$ and the position of the center of circle $\left(x_{c}, y_{c}\right)$ obtained from the circular fit mentioned earlier.

$$
\theta_{H}^{f}=\tan ^{-1}\left(\frac{T S_{y_{l p}^{f}}^{f}-T S_{y_{c}}}{T S_{x_{l p}}^{f}-T S_{x_{c}}}\right)
$$

As it is better to use the IMU yaw angle reading to fully capture the actual angular turning rate of the rover, we need to shift the IMU reading by $\left(\theta_{H}^{f}-\psi^{f}\right)$, where $\psi^{f}$ is the last IMU yaw measured for the test. The $\theta_{H}$ angle (correcting the yaw measurement $\psi$ ) can then be found at each time using the following equation:

$$
\theta_{H}=\left(\theta_{H}^{f}-\psi^{f}\right)+\psi
$$

This $\theta_{H}$ angle is then used in the transformation matrix to obtain the position of all four wheels, at each time. Eq 5.6-5.9 summarize the equations to obtain these coordinates, in the total station frame.

$$
\begin{aligned}
& {\left[\begin{array}{l}
T S_{x_{i f}} \\
T S_{y_{i f}}
\end{array}\right]={ }_{L P}^{T S} T\left[\begin{array}{c}
-\delta x_{l p}-B / 2 \\
L / 2
\end{array}\right]} \\
& {\left[\begin{array}{l}
T S_{x_{i b}} \\
T S_{y_{i b}}
\end{array}\right]={ }_{L P}^{T S} T\left[\begin{array}{c}
-\delta x_{l p}-B / 2 \\
-L / 2
\end{array}\right]} \\
& {\left[\begin{array}{l}
T S_{x_{o f}} \\
T S_{y_{o f}}
\end{array}\right]={ }_{L P}^{T S} T\left[\begin{array}{c}
-\delta x_{l p}+B / 2 \\
L / 2
\end{array}\right]} \\
& {\left[\begin{array}{c}
T S x_{x_{o b}} \\
T S_{y_{o b}}
\end{array}\right]={ }_{L P}^{T S} T\left[\begin{array}{c}
-\delta x_{l p}+B / 2 \\
-L / 2
\end{array}\right]}
\end{aligned}
$$

The magnitude and orientation of $T S_{v_{i f}}, T S_{v_{i b}}, T S_{v_{o f}}$ and ${ }^{T S} v_{o b}$ velocities at each time $t$ are then derived from each of the wheels position data, knowing the time interval between measurements $(\delta t)$ and following the format:

$$
\left\|^{T S} v_{i f}(t)\right\|=\frac{\sqrt{\left(T S_{x_{i f}(t)-T S} x_{i f}(t-1)\right)^{2}+\left({ }^{T S} y_{i f}(t)-T S y_{i f}(t-1)\right)^{2}}}{\delta t}
$$




$$
\angle^{T S} v_{i f}(t)=90^{\circ}+\tan ^{-1}\left(\frac{T S y_{i f}(t)-{ }^{T S} y_{c}}{T S x_{i f}(t)-T S_{x_{c}}}\right),
$$

where the if subscript can be replaced by $o f, i b$ and $o b$ to compute the magnitude and orientation of the velocity for the outer front, inner back and outer back wheels respectively.

To simplify the model, an average of the magnitude of the front and back velocities is taken for the remainder of the procedure. The magnitude of the inner and outer wheels actual velocities, i.e. $\left\|v_{i}^{A C T}\right\|$ and $\left\|v_{o}^{A C T}\right\|$, is defined as

$$
\begin{gathered}
\left\|v_{i}^{A C T}\right\|=\frac{\left\|^{T S} v_{i f}\right\|+\left\|^{T S} v_{i b}\right\|}{2}, \\
\left\|v_{o}^{A C T}\right\|=\frac{\left\|T S_{v_{o f}}\right\|+\left\|^{T S} v_{o b}\right\|}{2},
\end{gathered}
$$

To reduce the effect of noise in the measurements, the resulting actual velocity is filtered through a moving average filter with a window size of 3 , starting at the third sample of the set. Fig $5.5 \mathrm{~b}$ shows the effect of the filter on the inner and outer wheels' actual velocities, for the case of an $R^{\prime}$ turn.

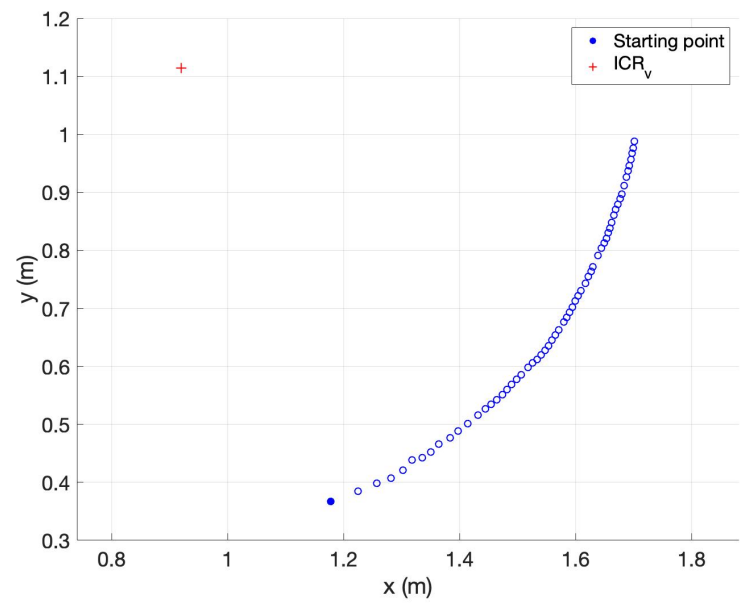

(a)

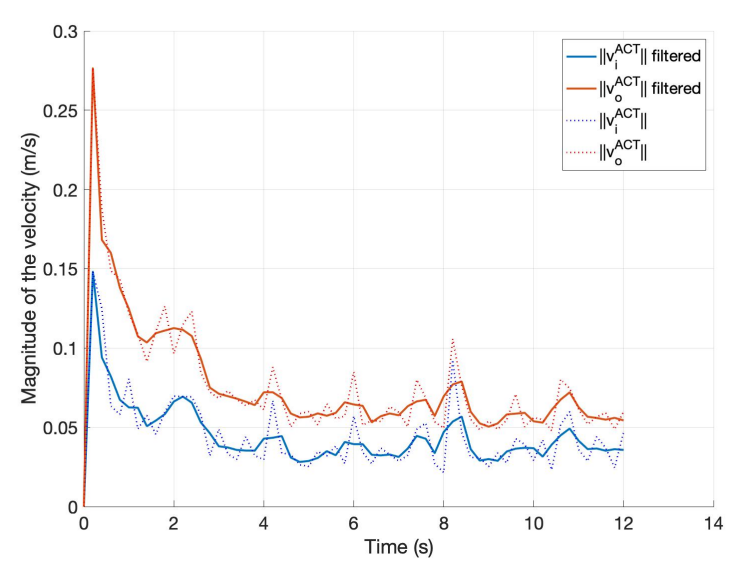

(b)

Figure 5.5 For a $R^{\prime}$ turn, this figure is showing the a) position data with the estimated position of $I C R_{v}$ and b) inner and outer wheels' velocity profile before and after moving average filtering. 


\subsection{Torque Comparison}

To investigate the contributing factors to the increasing power consumption of skid-steered wheeled robots on loose soil, the special set of turning radii identified in the Section 2.1.2 is closely examined. As mentioned, in this special case, the inner wheels' slip angle $\left(\beta_{i}\right)$ is always greater than $90^{\circ}$, since the projection of the actual velocity of the inner wheels $\left(v_{i}^{A C T}\right)$ along the vehicle's $Y$-axis is in the opposite direction to the wheel linear velocity $\left(v_{i}^{C M D}\right)$. When operating on loose soil, this means that the wheel is excavating soil in a portion of the area where it is being dragged into. In this section, a torque proportional curve is drawn for each turns to see if the torque applied by the inner wheel is higher in the special case.

For any electromechanical system, the input electrical power $P$ equals the output mechanical power $P_{\text {out }}$ such that $P=\eta_{e} P_{\text {out }}=\eta_{e} \tau \omega$, where $\eta_{e}$ is the power efficiency factor, $\tau$ is the output torque and $\omega$ is the angular velocity of the wheel in this case. Assuming a constant efficiency, the measurements gathered in the set of $90^{\circ}$ turns can be used to draw a torque-proportional curve knowing the following:

$$
\tau \propto \frac{P}{\omega}
$$

Fig. 5.6 shows the inner and outer wheels torque-proportional curve using the power measurements and the commanded angular velocity $\left(\omega^{C M D}\right)$. For the $2 R^{\prime} / 3$ case, which corresponds to the turning radius in the special case range presented in Section 2.1.2, the inner wheels' torque is clearly isolated as it requires around $70 \%$ more torque for these wheels to rotate versus the $R^{\prime} / 3$ case. 

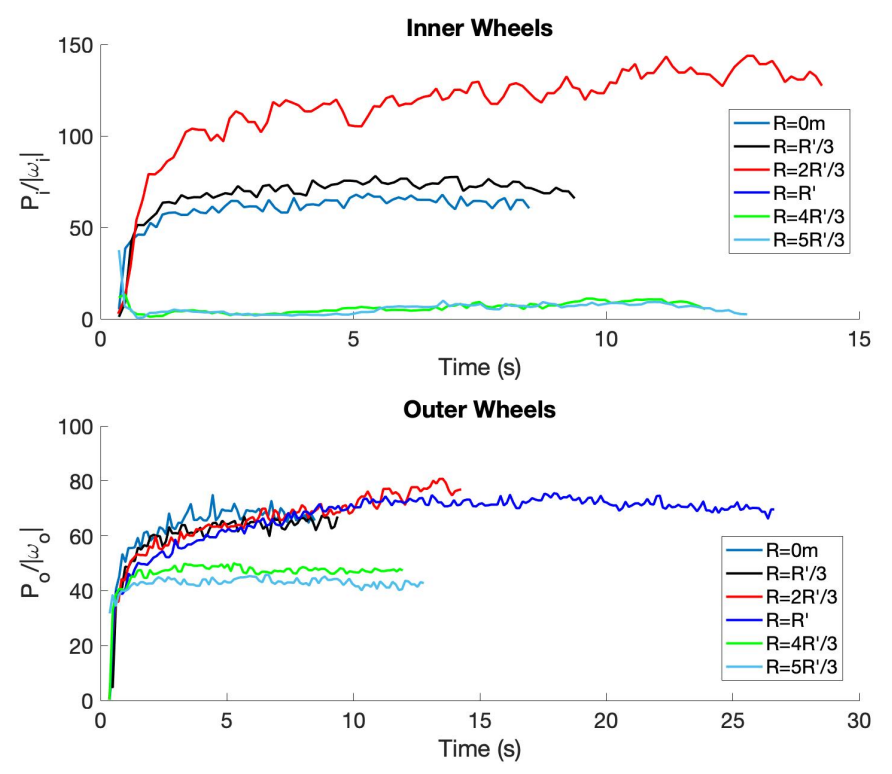

Figure 5.6 Inner (top) and outer (bottom) wheels torque-proportional curve following Eq. 5.14. For the inner wheels, the $2 R^{\prime} / 3$ case significantly needs more torque than the other radii in order to achieve its desired turn.

The significant increase in measured torque for the $2 R^{\prime} / 3$ case is most likely due to the fact that the grousers need to excavate the sand in front of the wheel. As the wheel progresses and pushes the sand pile, it becomes harder for the grousers to excavate sand from under the wheel to the front, hence requiring more torque to rotate the wheel. A one-dimension schematic comparison of a normal sand excavation process (when the wheel moves out of the region where sand was excavated) and a scenario when the sand is being compacted in front of the wheel is depicted in Fig. 5.7. As more torque is required by the motor to rotate the wheel, the system needs to consume an additional amount of electrical power. Therefore, a first argument to explain the peak in power consumption shown in Fig. 4.10 is identified. 


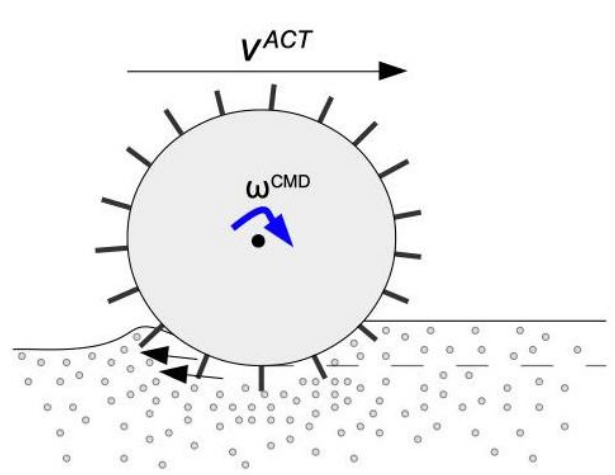

(a)

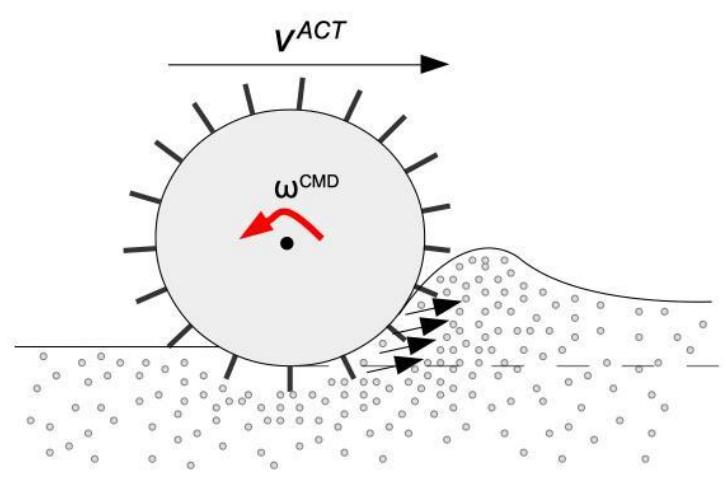

(b)

Figure 5.7 Schematic comparison of a) the usual excavation process by the wheel angular rotation $\left(\omega^{C M D}\right)$ is aligned with the wheel progression $\left(v^{A C T}\right)$, i.e. when $\beta=0^{\circ}$, and (b) the excavation process when the wheel rotation is opposite to the wheel progression, i.e. when $\beta=180^{\circ}$.

Future work would be to model the terramechanics of the soil shear failure when a wheel is excavating a growing pile of sand, as shown in Fig. 5.7b. Understanding the interactions between the wheel and the soil in this special case will help to estimate the amount of torque required for the skid-steer rover's inner wheels to push the sand and hence predict their power consumption in the $B / 2<R<R^{\prime}$ range of turning radii.

\subsection{Sinkage}

Aspects of a rover power's consumption may be related to how much its wheels are sunk into the soil, and time-dependant power phenomena (described in Section 4.4.1) may be related to the time dependency of sinkage. Further, as analysis of slip angles shows, wheel-soil interactions for skid-steer rovers are not purely longitudinal, but also include lateral components, and are thus 2D.

As shown in Section 1.1.4, the wheel sinkage models found in the literature are only based on longitudinal slip $s_{y}$, meaning that the effects of a wheel skidding laterally are not considered. Another limitation is that the longitudinal slip definition is not suitable for the inner wheels in the special case region of turning radii, i.e. $B / 2<R<R^{\prime}$, as it assumes that the projection of the actual velocity of the wheel, $v^{A C T}$, onto the axis of the commanded wheel velocity, $v^{C M D}$, and the $v^{C M D}$ velocity itself are in the same direction (i.e. $\beta<90^{\circ}$ ). Thus, the inner wheels' sinkage during these turns can't be modeled using the slip-sinkage models presented earlier in Section 1.1.4. These models are also only estimating the steady-state sinkage as none of them include a time dependency.

These limitations motivate the need to develop a new sinkage model that can estimate the 
sinkage of a wheel with respect to time, considering its longitudinal and lateral slip. This 2D slipsinkage model is presented in this section, along with an experimental validation of the predictions using the data gathered during the Husky turns and with the single-wheel test bed.

Throughout this section, the inner and outer wheel sinkage is studied, but also the overall sinkage of the skid-steer rover, defined as the sinkage at the geometric center of the rover and calculated by averaging the inner and outer wheel sinkage values.

\subsubsection{D Slip-Sinkage Model}

The idea is to design a semi-empirical slip-sinkage model that estimates the volume of sand that is excavated out from under the wheel $\left(V_{R E M}\right)$ and the volume of sand that is being added and

compressed underneath the wheel $\left(V_{A D D}\right)$ as it is moving along the $v^{A C T}$ velocity vector. Knowing the wheel contact patch area at each time, this simplistic model based on wheel geometry and soil properties would be able to provide an estimate of a wheel sinkage with respect to time. In this section, the procedure for this first semi-empirical two-dimensional slip-sinkage model is explained. Then, the model parameters are tuned using a set of tests with the single-wheel test bed, and the data gathered during the Clearpath Husky turns (and processed following Section 5.1) is used to estimate the inner and outer wheel sinkage.

\section{Procedure}

As mentioned, this 2D slip-sinkage model is semi-empirical; the sinkage prediction relies on the experimental data gathered and processed in Section 5.1. Accordingly, the model takes the inner and outer wheels' actual velocity, $v_{i}^{A C T}(t)$ and $v_{o}^{A C T}(t)$ respectively, as well as the inner and outer slip angle, $\beta_{i}$ and $\beta_{o}$ respectively, for the turn as input. The procedure described here covers the modeling of the sinkage for a single wheel; the same procedure is used on the inner and outer wheels independently. Fig. 5.8 shows the top and side view of a wheel moving at an actual velocity $v^{A C T}$ with a commanded linear velocity $v^{C M D}=r \omega^{C M D}$; the angle between the two velocities is the slip angle $\beta$. Similar to the work presented in Section 1.1.4, the sinkage $d$ is the vertical distance between the bottom of the wheel and the undisturbed soil height in front of the wheel. 


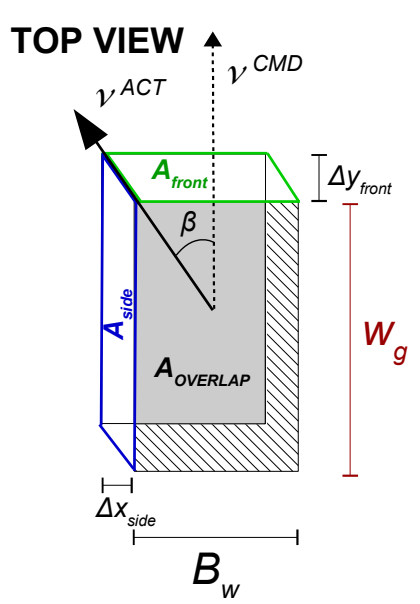

(a)

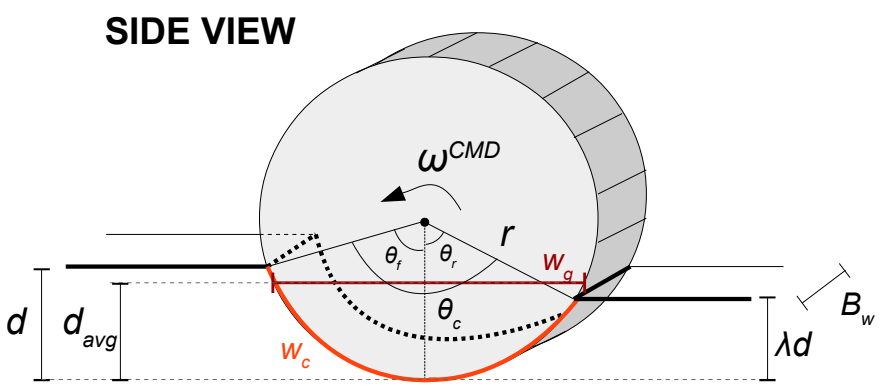

(b)

Figure 5.8 a) Top view and b) side view of the wheel modeled.

To update the sinkage value at time $t$, the first step of the procedure is to compute the front and rear entrance angle of the wheel into the soil, $\theta_{f}$ and $\theta_{r}$ respectively (see Fig. 5.8b), knowing the previously computed sinkage $d(t-\delta t)$ :

$$
\begin{gathered}
\theta_{f}=\cos ^{-1}\left(1-\frac{d(t-\delta t)}{r}\right), \\
\theta_{r}=\cos ^{-1}\left(1-\frac{\lambda d(t-\delta t)}{r}\right),
\end{gathered}
$$

where $r$ is the wheel radius and $\lambda$ is the wheel sinkage ratio, which is assumed constant throughout the wheel motion. The sum of these angles, i.e. $\theta_{c}$, is then used to compute the length of the contact patch $w_{c}$ following:

$$
w_{c}=r \theta_{c}=r\left(\theta_{f}+\theta_{e}\right)
$$

For pneumatic tires which are rounded, the thickness of the wheel contact patch area $\left(B_{c}\right)$ is larger than the actual thickness of the wheel $\left(B_{w}\right)$, as seen in Fig. 5.9a. The relation between the wheel contact patch thickness and actual wheel thickness is expressed as

$$
B_{c}=e_{c w} B_{w}
$$

where $e_{c w}>1$ for pneumatic tires and $e_{c w}=1$ for rigid cylindrical wheels. The value of the $e_{c w}$ 
ratio is found to be around 1.05 for the Husky wheel. Subsequently, the volume of sand being excavated (or removed) from under the wheel during the time interval $\delta t$ can be estimated as:

$$
V_{R E M}=k_{E X} B_{c} h v^{C M D} \delta t
$$

where $k_{E X}$ is the excavation efficiency parameter. An estimation of this parameter can be computed using the grouser geometry, following the equation:

$$
k_{E X}=1-\frac{A_{\text {grousers }}}{2 \pi r B_{c}}=1-\frac{n_{g} B_{g} l_{g}}{2 \pi r B_{c}},
$$

where $n_{g}$ is the number of grousers on the wheel, $B_{g}$ and $l_{g}$ is the width and thickness of each grouser respectively (as depicted in Fig. 5.9b, for a Husky wheel).

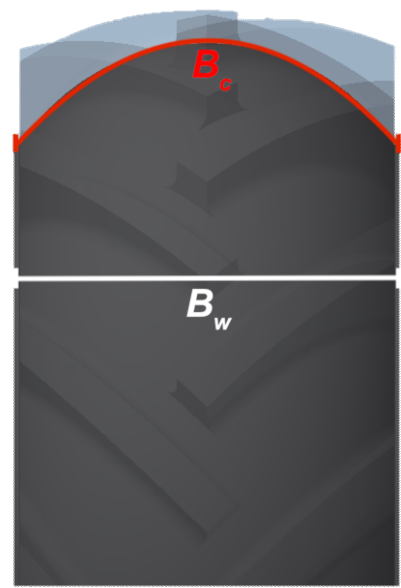

(a)

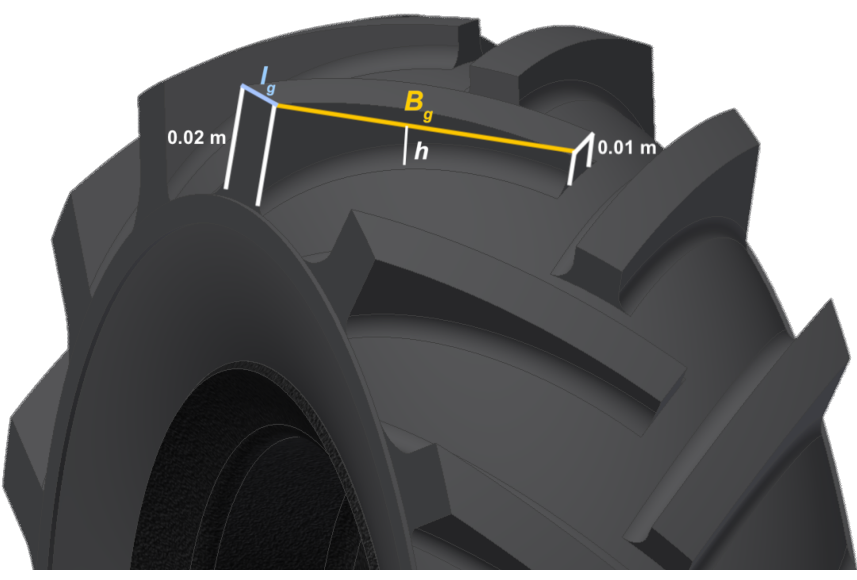

(b)

Figure 5.9 Husky wheel design showing the important parameters used to model the wheel.

Now that the $V_{R E M}$ volume is known, the next step is to estimate the volume of sand in front of the wheel $\left(V_{\text {front }}\right)$ and on the side of the wheel $\left(V_{\text {side }}\right)$, as shown in Fig. 5.8a. First, the wheel displacement on the front $\Delta y_{\text {front }}$ and on the side $\Delta x_{\text {side }}$ is calculated knowing each wheel's actual velocity magnitude at each time $t$, namely $\left\|v^{A C T}(t)\right\|$ or simply $v^{A C T}$, the slip angle $\beta$, and the time interval $\delta t$ before the next measurement.

$$
\Delta y_{\text {front }}=v^{A C T} \delta t \cos \beta
$$




$$
\Delta x_{\text {side }}=v^{A C T} \delta t \sin \beta
$$

Using the displacement distance in the two directions, the volume of sand in front and on the side of the wheel is computed following:

$$
\begin{gathered}
V_{\text {front }}=A_{\text {front }} \times d(t-1)=\Delta y_{\text {front }} \times B_{w} \times d(t-\delta t), \\
V_{\text {side }}=A_{\text {side }} \times d(t-\delta t)=\Delta x_{\text {side }} \times w_{g} \times d(t-\delta t),
\end{gathered}
$$

where $w_{g}$ is the length of the wheel along the sand (see Fig. 5.8a). This distance is computed with the average of the front and rear sinkage, namely $d_{\text {avg }}(t)$ or simply $d_{\text {avg }}$, using Eq. 5.25 and 5.26.

$$
\begin{gathered}
d_{\text {avg }}=\frac{(1+\lambda) d}{2} \\
w_{g}=2 \sqrt{d_{\text {avg }}\left(2 r-d_{\text {avg }}\right)}
\end{gathered}
$$

These $V_{\text {front }}$ and $V_{\text {side }}$ volumes are then multiplied by the sinkage parameters $k_{f}$ and $k_{s}$ respectively. These two sinkage parameters need to be tuned as they depend on the soil properties and wheel design. Since the sand on the side of the wheel is partially being pushed (or bulldozed), the $k_{s}$ constant should be smaller that $k_{f}$, i.e. $k_{s}<k_{f}$.

$$
V_{A D D}=k_{f} V_{\text {front }}+k_{s} V_{\text {side }}
$$

Finally, when $V_{A D D}$ and $V_{R E M}$ are know at time $t$, the sinkage can be updated by dividing the difference between the two volumes over the wheel contact patch area, i.e. $B_{c} w_{c}$.

$$
d(t)=d(t-\delta t)+\frac{V_{R E M}-V_{A D D}}{B_{c} w_{c}}
$$

To start the iterative process, the inital sinkage is set to the static sinkage, i.e. $d(0)=d_{0}$. Then, at each iteration, the sinkage value is updated using the magnitude of the new $v^{A C T}$ vector and following Eq. 5.21-5.28. Saturation bounds are also fixed for the model; at any time, the sinkage can't be lower than the static sinkage $d_{0}$ or higher than the ground clearance of the rover $\left(h_{g c}\right)$ ). 


\section{Model Validation}

The 2D slip-sinkage was validated using a Husky pneumatic wheel and the single-wheel test bed presented in Section 3.3. The tuned model is then used to predict the inner and outer wheel sinkage during the set of turns with the Husky in the laboratory sandbox. To begin, the geometry of this wheel is presented in Table 5.1.

Table 5.1 Husky Wheel Geometry and Model Parameters

\begin{tabular}{ll}
\hline Parameters & Value \\
\hline Wheel radius $(r)$ & $0.165 \mathrm{~m}$ \\
Wheel thickness $\left(B_{w}\right)$ & $0.125 \mathrm{~m}$ \\
Number of grousers $\left(n_{g}\right)$ & 28 \\
Grouser height $(h)$ & $0.015 \mathrm{~m}$ \\
Grouser width $\left(B_{g}\right)$ & $0.085 \mathrm{~m}$ \\
Grouser thickness $\left(l_{g}\right)$ & $0.011 \mathrm{~m}$ \\
Ground clearance $\left(h_{g c}\right)$ & $0.13 \mathrm{~m}$ \\
\hline Static sinkage $\left(d_{0}\right)$ & $0.01 \mathrm{~m}$ \\
Wheel sinkage ratio $(\lambda)$ & 0.7 \\
\hline
\end{tabular}

Using the wheel geometry, the excavation efficiency $k_{E X}$ is estimated with the parameters shown in Table 5.1 and Eq. 5.20. The value obtained for the Husky wheel is $\boldsymbol{k}_{E X}=\mathbf{0 . 8 0 7 6}$. Subsequently, the next step is to tune the two other sinkage parameters, i.e. $k_{f}$ and $k_{s}$, with a set of two linear paths (see Fig. 3.6b) with the single-wheel test bed. With constant $v^{C M D}$ and $v^{A C T}$ velocities, the soil compaction in front parameter $k_{f}$ is tuned when the slip angle $\beta=0^{\circ}$ (as $V_{\text {side }}=0 \mathrm{~m}^{3}$ ) and the soil compaction on the side parameter $k_{s}$ is tuned when the slip angle $\beta=90^{\circ}$ (as $V_{\text {front }}=0$ $\mathrm{m}^{3}$ ). The results when $v^{C M D}=0.08 \mathrm{~m} / \mathrm{s}$ and $v^{A C T}=0.02 \mathrm{~m} / \mathrm{s}$ along with the predicted sinkage with the tuned parameters is shown in Fig. 5.10.

Furthermore, the static sinkage $d_{0}$ of the Husky wheel on the GRC-1 soil simulant is determined experimentally using the single-wheel test bed under a load equivalent to 1/4 of the Husky's mass. The linear potentiometer is used to measure distance between the soil level and the wheel after applying the vertical load. 


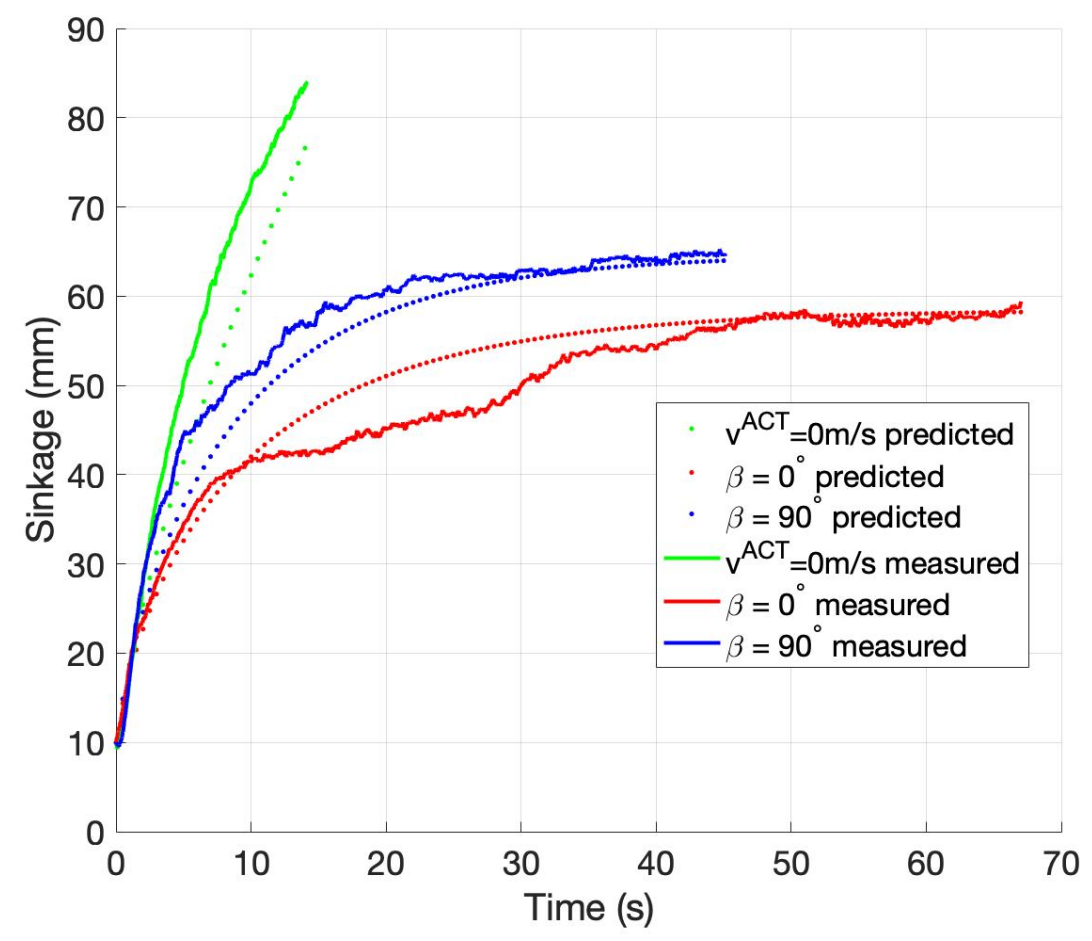

Figure 5.10 Measured and predicted sinkage during three linear arc path tests with the singlewheel test bed with $v^{C M D}=0.08 \mathrm{~m} / \mathrm{s}$. The actual velocity was set to $0.02 \mathrm{~m} / \mathrm{s}$ for the $\beta=0^{\circ}$ and $\beta=90^{\circ}$ tests (red and blue line respectively) and to $0 \mathrm{~m} / \mathrm{s}$ for the $100 \%$ slip tests (green line). The latter case was performed to confirm the $k_{E X}$ estimation of Eq. 5.20.

To obtain these predicted sinkage curves of Fig. 5.10, the sinkage parameters were set to $k_{f}=\mathbf{0 . 8 5}$ and $\boldsymbol{k}_{\boldsymbol{s}}=\mathbf{0 . 3 5}$. As expected, $k_{s}<k_{f}$ due to the fact that the added volume of soil on side of the wheel is partially being pushed away whereas the added volume soil on the front is moving more efficiently beneath the wheel, being helped by the grousers. The estimated value of $k_{E X}$ was also confirmed with a $100 \%$ slip test, where $v^{A C T}=0 \mathrm{~m} / \mathrm{s}$ and therefore $V_{A D D}=0 \mathrm{~m}^{3}$. Although the prediction shown in Fig. 5.10 is for $v^{A C T}=0.02 \mathrm{~m} / \mathrm{s}$, the accuracy of the prediction is reduced when the actual velocity is doubled, i.e. $v^{A C T}=0.04 \mathrm{~m} / \mathrm{s}$. At this speed, the sinkage parameters should be at around $k_{s} \approx 0.2$ and $k_{f} \approx 0.6$. This means that the sinkage parameters have a dependency to the actual velocity of the wheel; they should decrease when $v^{A C T}$ increases.

Once the sinkage parameters are tuned, the model is now ready to estimate the inner and outer wheel sinkage of a skid-steered wheeled robot during a turn. Using the data gathered and processed for the set of Husky turns presented in Section 4.4, the $v_{i}^{A C T}(t)$ and $v_{o}^{A C T}(t)$ functions as well as the actual slip angles ( $\beta_{i}$ and $\beta_{o}$ ) are injected into the model. Fig. 5.11 shows the resulting $d_{i}(t)$ and $d_{o}(t)$ sinkage predicted for the inner and outer wheels respectively. For the inner wheel sinkage when $R=2 R^{\prime} / 3$, it should be mentioned that the $2 \mathrm{D}$ slip-sinkage model does not at this point take 
into account the effects of the wheel rotating in opposite direction to the motion, i.e. the fact that $\beta>90^{\circ}$. Tests in the single-wheel test bed demonstrated that inverting the wheel rotation results in higher sinkage. It was also noticed that a wheel progressing with an actual velocity of $v^{A C T}=0.02$ $\mathrm{m} / \mathrm{s}$ and rotating at $0.08 \mathrm{~m} / \mathrm{s}$ in the opposite direction $\left(\beta=180^{\circ}\right)$ sank faster than a wheel rotating at the same speed with $100 \%$ slip (see Appendix B for more single-wheel test results).
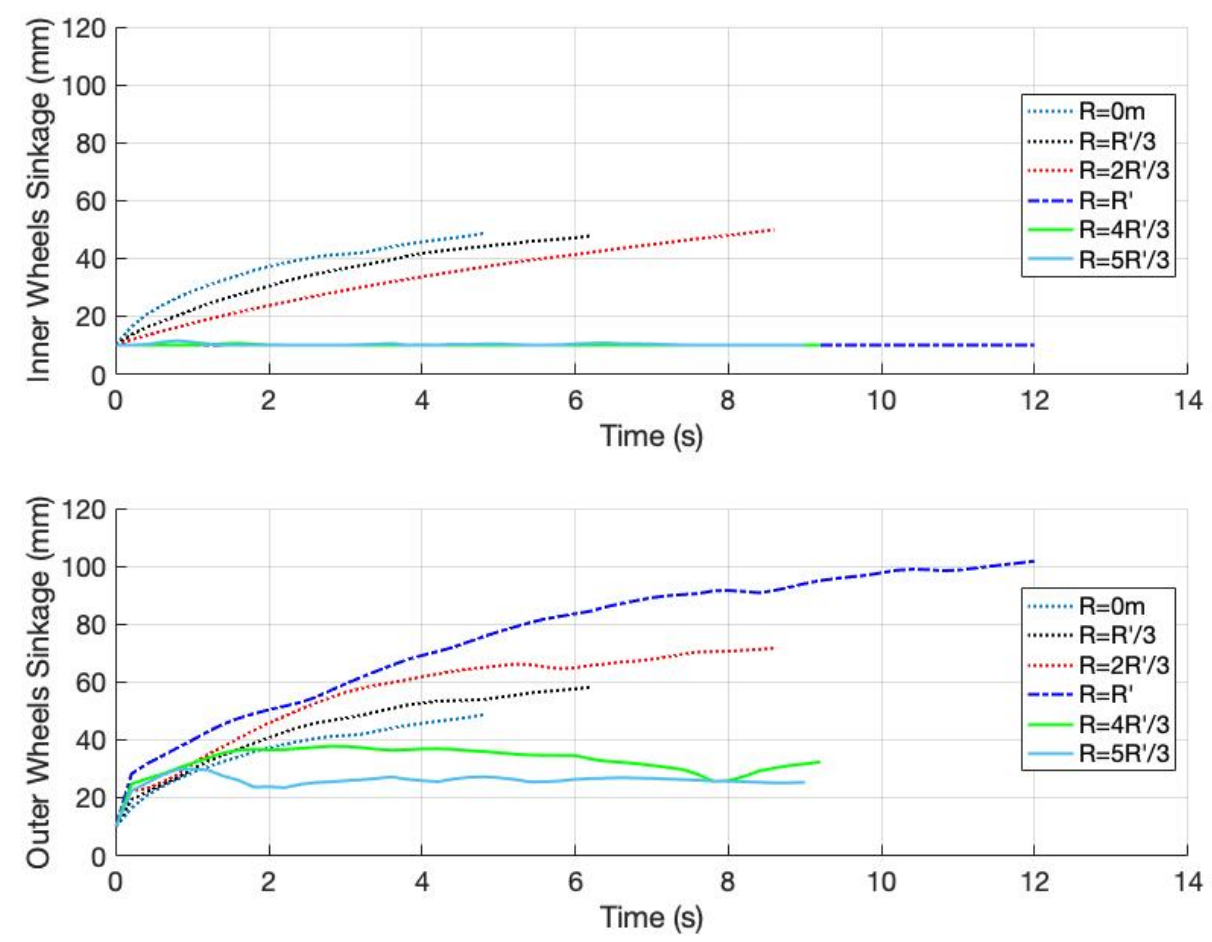

Figure 5.11 Estimated inner (top) and outer (bottom) wheel sinkage over time for the set of turning radii tested with the Husky in the sandbox.

The predicted sinkage value at the end of the each $90^{\circ}$ turn is then plotted as a function of turning radius in Fig. 5.12. In this figure, it is clear that the outer wheels' sinkage peak at $R^{\prime}$ although the overall sinkage, or the sinkage at the geometric center, peaks at $2 R^{\prime} / 3$, the turning radius in the special case region identified. This prediction is obtained even though the model doesn't yet account for the added inner wheel sinkage caused by wheel rotating in opposite direction to the motion. In spite of this, the resulting overall sinkage curve still reproduces the trend of the average power consumes during a turn as a function of turning radius (seen in Fig. 4.10), which is a promising result. 


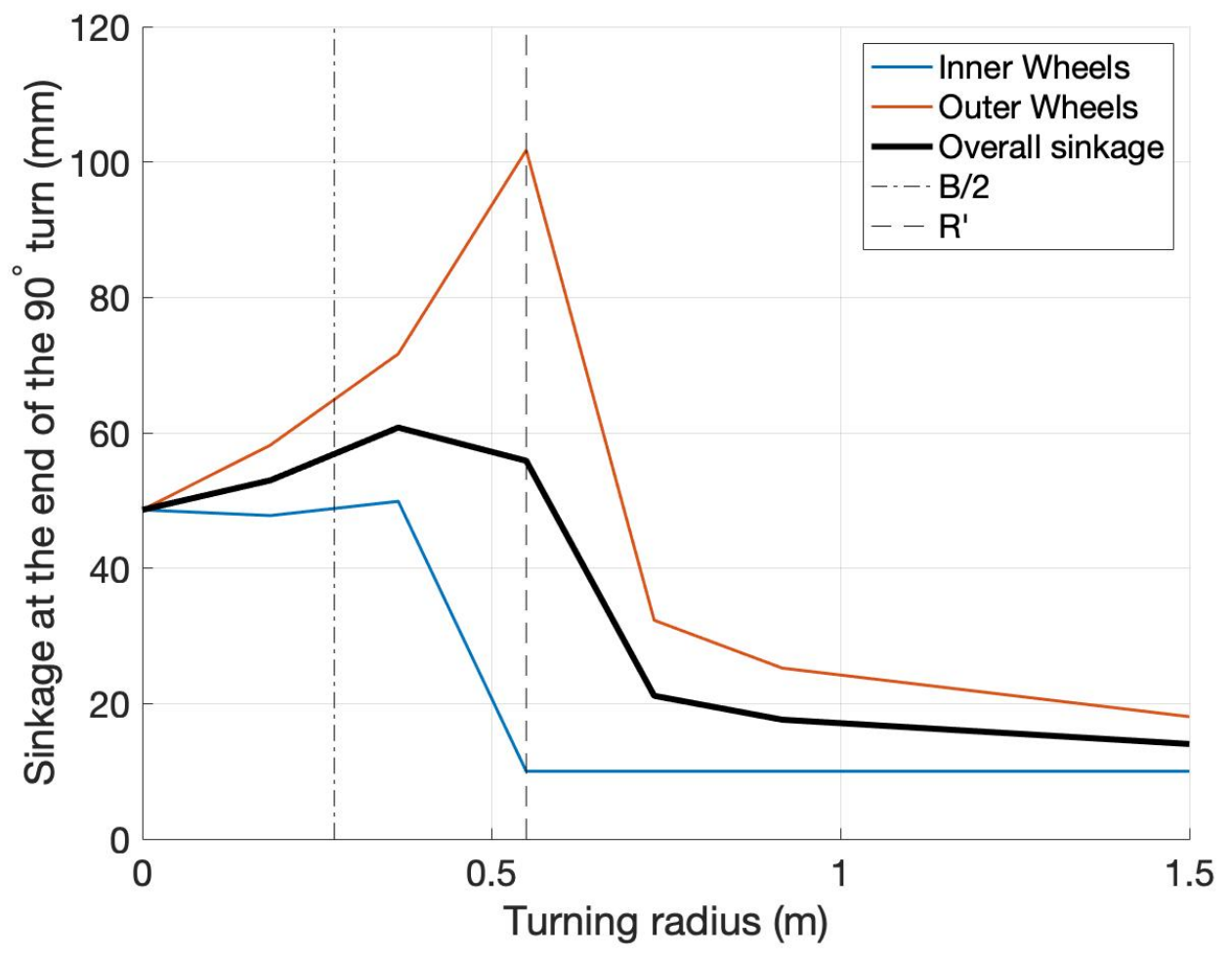

Figure 5.12 Resulting prediction of the inner (blue) and outer (red) wheel sinkage at the end of the $90^{\circ}$ rotation, with varying turning radius. The overall sinkage, i.e. the sinkage at the geometric center of the vehicle, is also shown and seems to follow the same trend as power vs. $R$ curve seen in Fig. 4.10.

\subsubsection{Comparing the predicted and measured sinkage}

Even though the model predicts sinkage that follows the overall observations made during the test campaigns, a detailed validation using the data gathered should also be made. In this section, the outer wheels' sinkage is first measured with the total station tracking of the landmark prism placed along the edge of the rover chassis, at the midpoint between the front and back outer wheels, in the case of left turns. On the other side, the inner wheels' sinkage is measured by reproducing their arc trajectory with the single-wheel test bed. 


\section{Outer wheels}

Since the landmark prism lies only $6.25 \mathrm{~cm}$ from the midpoint between the outer wheels (i.e. $B / 2-\delta x_{l p}$ ), is was assumed that the sinkage (or variation along total station $Z$-axis) was equivalent to the outer wheels' sinkage. The relative variation in the ${ }^{T S_{z_{l p}}}$ was considered, by substracting at each measurement by the initial value. To facilitate the comparison with the predicted sinkage, the curve shown in Fig. 5.13 starts at the static sinkage $d_{0}$ value.

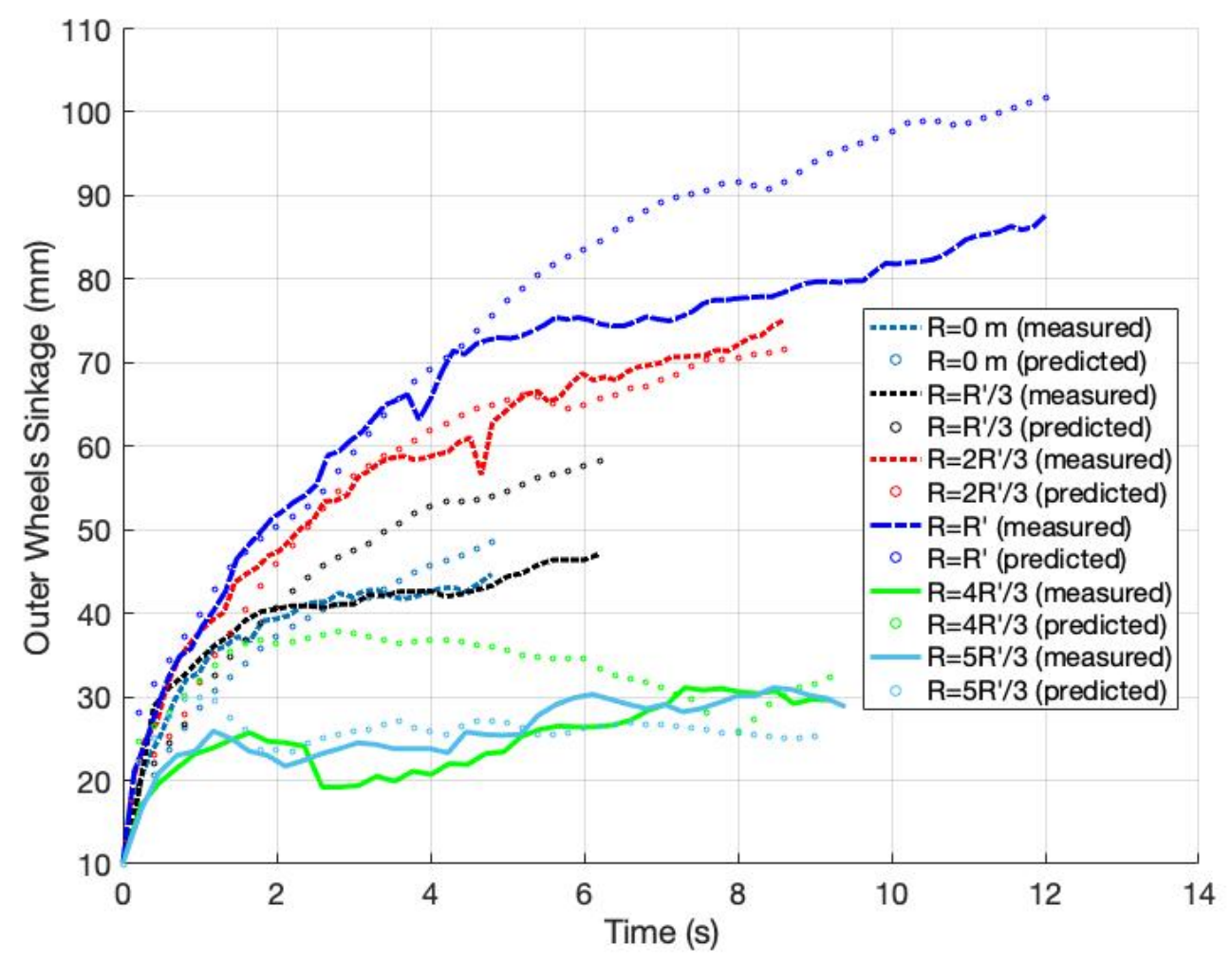

Figure 5.13 Measured outer wheel sinkage over time for the set of turning radii tested with the Husky in the sandbox. The outer wheel sinkage was estimated using the variations in the total station measurements of the landmark prism along the $z$ axis, i.e. ${ }^{T S} \Delta z_{l p}$.

If Fig. 5.13 is compared to the outer wheels' predicted sinkage shown in Fig. 5.11 (and reproduced again in Fig. 5.13), the relative trends between the tests is approximately the same, i.e. the sinkage at the end of the turn increasing from $R=0 \mathrm{~m}$ to $R^{\prime}$ and then dropping below the point turn sinkage for $R>R^{\prime}$. The magnitude of the sinkage is similar, although the model seems to be slightly overestimating the wheel sinkage (especially for the $R^{\prime} / 3$ and $R^{\prime}$ turn). It can be concluded that the model is successfully predicting the outer wheel's sinkage to some extent, as this is a first version of a semi-empirical two-dimensional slip-sinkage model and some key improvements to be made are already indentified. 
It was also attempted to estimate the inner wheel sinkage knowing the distance between the landmark prism and the inner track, the vehicle's roll and the outer wheel sinkage. However, this result yielded to no clear conclusion due to the noise in the small roll values measured $\left(\phi<5^{\circ}\right)$ and the variability in the flatness of the soil.

\section{Inner wheels}

As there is no sinkage estimation for the inner wheels in the Husky turns data, further tests using the five-axis single-wheel test bed were executed to investigate the sinkage of the Husky's inner wheels by reproducing their motion during a turn of radius $R$. Table 5.2 presents the inner wheel's turning radius $\left(R_{i}\right)$, slip angle $\beta$, tangential velocity $\left(v^{A C T}\right)$ and commanded wheel linear velocity $\left(v^{C M D}\right)$ used to replicate an inner wheel trajectory for each turning radius $R$ tested.

Table 5.2 Parameters to reproduce the Husky's inner wheel trajectories tested

\begin{tabular}{lllll}
\hline$R$ & $R_{i}$ & $\beta$ & $v^{A C T}$ & $v^{C M D}$ \\
\hline$R^{\prime} / 3$ & $0.141 \mathrm{~m}$ & $60^{\circ}$ & $0.057 \mathrm{~m} / \mathrm{s}$ & $0.17 \mathrm{~m} / \mathrm{s}$ \\
$2 R^{\prime} / 3$ & $0.267 \mathrm{~m}$ & $100^{\circ}$ & $0.042 \mathrm{~m} / \mathrm{s}$ & $0.08 \mathrm{~m} / \mathrm{s}$ \\
$R^{\prime}$ & $0.6127 \mathrm{~m}$ & $40^{\circ}$ & $0.05 \mathrm{~m} / \mathrm{s}$ & $0 \mathrm{~m} / \mathrm{s}$ \\
$4 R^{\prime} / 3$ & $0.795 \mathrm{~m}$ & $25^{\circ}$ & $0.127 \mathrm{~m} / \mathrm{s}$ & $0.07 \mathrm{~m} / \mathrm{s}$ \\
\hline
\end{tabular}

The sinkage measured by the linear string potentiometer for the four test cases is reported in Fig. 5.14. The first observation that should be made is that the wheel sinks more when $R \leq R^{\prime}$ than for the $4 R^{\prime} / 3$ case, which is consistent with relative power consumption observed below and above $R^{\prime}$ in Section 4.4 .

It should also be noted that for the $2 R^{\prime} / 3$ turn, the sinkage is greater than in the $R^{\prime} / 3$ turn, even though the wheel is rotating more than twice as fast in the latter case. This additional sinkage means that there is also an additional amount of sand accumulation next to the inner wheels, which results in an increasing power required not only to drag the inner wheels but also to bulldoze the sand pile next to the wheels. 


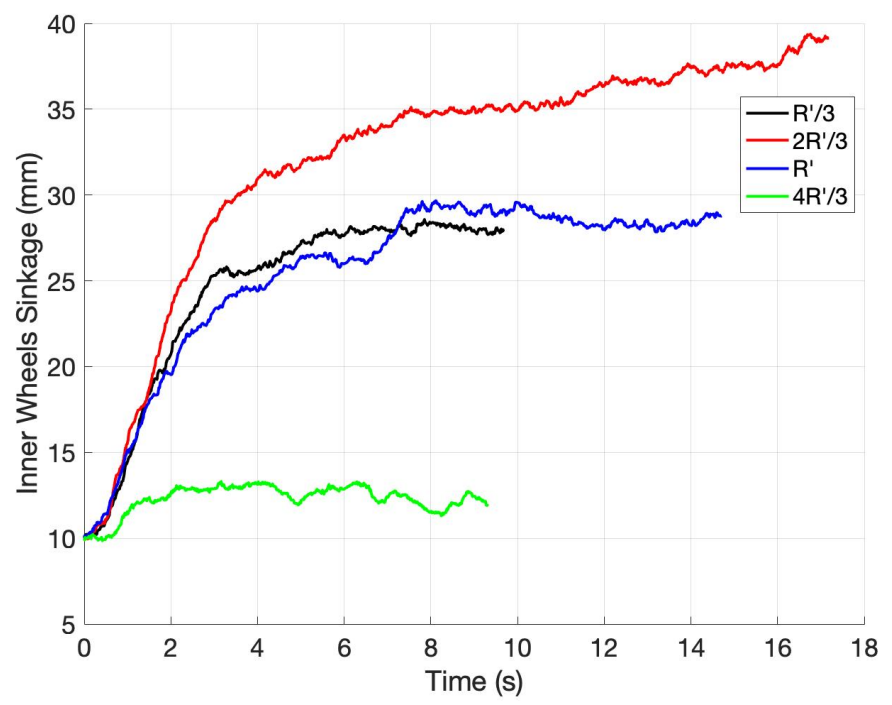

Figure 5.14 Measured sinkage versus time for the set of turning radii tested (Table 5.2). The wheel sinks more in the $2 R^{\prime} / 3$ case than in the $R^{\prime} / 3$ even if its rotation speed is less than half. The static sinkage of $d_{0}=10 \mathrm{~mm}$ was added to all the measurements, to compare with the sinkage prediction of Fig. 5.11.

Compared to the model predictions shown in Fig. 5.11, the $2 R^{\prime} / 3$ turn measured sinkage is higher than the sinkage recorded for the $R^{\prime} / 3$ and $R^{\prime}$ turn, which is not the case for the modeled sinkage. This result can be explained by the fact that the $2 \mathrm{D}$ slip-sinkage model not taking into account the effect of a wheel rotation opposed to its motion. However, the model doesn't properly estimate the inner wheel sinkage at $R^{\prime}$, which is in the same vicinity as the $R^{\prime} / 3$ case. This is due to the fact that, for the model, the wheel can only sink if sand is being excavated by wheel rotation, i.e. $v^{C M D} \neq 0$. Future work thus should include accounting for both the added sinkage caused by wheel rotation opposed to its motion and when $v^{C M D}=0$.

In conclusion, the 2D slip-sinkage model presented in this section shows promising results, especially when predicting the outer wheels' sinkage (which recorded more sinkage than the inner wheel), although some key improvements should be implemented moving forward. Here is a summary of these identified limitations of the model:

- The sinkage parameters, $k_{s}$ and $k_{f}$, should have a dependency to the actual wheel velocity $v^{A C T}$.

- The model should consider the added sinkage due to the wheel rotating in opposite direction to the projection of $v^{A C T}$ along the wheel, which occurs for the inner wheels in the special case region (when $B / 2<R<R^{\prime}$ ).

- The model should predict the sinkage when $v^{C M D}=0$, which only occurs for the inner wheels when $R=R^{\prime}$. 


\subsection{Sand Bulldozing Force}

With the sinkage estimation obtained in the previous section, the goal is to predict the added power required by the skid-steer rover to achieve its desired turn. To do so, the sand bulldozing force is first calculated using a simplified version of the Skonieczny model presented in Section 1.1.4. In this section, the simplifying assumptions are first presented before showing the procedure to compute the sand bulldozing force, $F^{S B}$, and the amount of power that needs to be added in the power model, i.e. $P^{A S}$. Finally, the $F^{S B}$ and $P^{A S}$ values are shown with respect to time and their average value during the turn are shown as a function of turning radius.

\section{Simplifying the Skonieczny Model}

As shown in Section 1.1.4, the sand surchage accumulation model developed by Skonieczny is specific to a wide-blade soil-tillage tool. However, it can be used in the case of a wheel that is moving with velocity $v^{A C T}$ and penetrating the soil by $d_{a v g}$, i.e. the average of the sinkage in the front and in the back of the wheel. The first simplifying assumption made is that the wheel is always perpendicular to the soil, i.e. $\alpha_{1}=90^{\circ}$. As the tests are performed in the cohensionless GRC-1 soil simulant, i.e. $c=0$, the model defined by Eq. 1.36 can be rewritten as:

$$
F^{S B}=w \frac{\rho g}{2}\left[\frac{d_{a v g} r_{\alpha}+r_{q}^{2} \tan \phi_{i}-I_{X_{C}} \rho g \frac{\left(r_{q}-d_{a v g} \cot \alpha_{2}\right)^{2}}{2\left(\cot \phi_{i}+\cot X_{C}\right)}}{-\sin \delta+\cos \delta \cot \left(X_{C}+\phi_{i}\right)}\right]
$$

where $F^{S B}$ is the sand bulldozing force, $\rho$ is the soil dry bulk density, $g$ is the gravitational acceleration, $\phi_{i}$ is the internal friction angle, $\delta$ is the external friction angle, $r_{\alpha}$ and $r_{q}$ are shown in Fig. 5.15, $X_{C}$ is the destructive angle, the Boolean indicator function $I_{X_{C}}$ is defined as follow

$$
I_{X_{C}}= \begin{cases}1, & r_{q}>d_{a v g} \cot X_{C} \\ 0, & r_{q} \leq d_{a v g} \cot X_{C}\end{cases}
$$

and $w$ is the width of the wheel perpendicular to the motion along the $X_{a}$-axis, which is computed using the slip angle $\beta$ and the width or the wheel on the soil (see Eq. 5.26):

$$
w=w_{g} \sin \beta
$$


Fig. 5.15 shows the modeled sand pile used to estimate the sand bulldozing force. This sand pile is modeled as a triangular prism with base $r_{q}$ and height $h_{q}$.

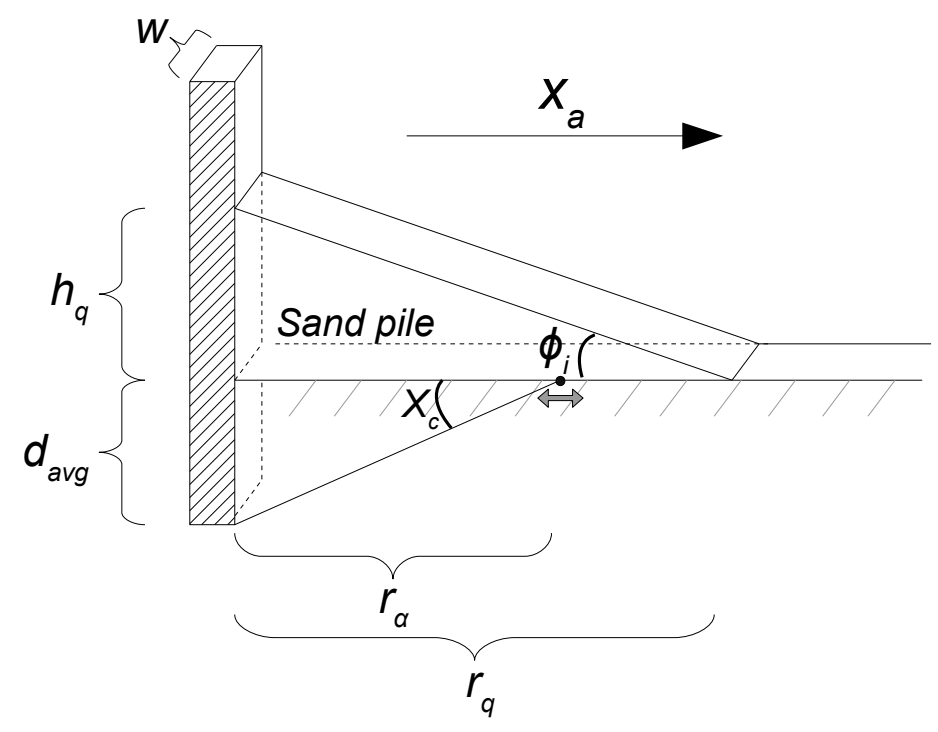

Figure 5.15 Side view of a projected wheel moving and bulldozing a sand pile along the $X_{a}$-axis.

From the geometry of the sand pile, the $r_{\alpha}$ value can be defined as:

$$
r_{\alpha}=d_{a v g} \cot X_{C}
$$

Also, the horizontal distance between the peak and front of the sand pile, i.e. $r_{q}$, depends on the volume of soil displaced at any point $x_{w}$ along the $X_{a}$-axis. This distance is defined as [28]:

$$
r_{q}=\sqrt{2 x_{w} d_{a v g} \cot \phi_{i}}
$$

where $x_{w}$ is the position of the wheel along the $X_{a}$-axis. Using Eq. 5.32 and knowing that for cohensionless soil, $r_{q}<r_{\alpha}$, the Boolean indicator function (Eq. 5.30) becomes simply 0, as $r_{q}<$ $d_{a v g} \cot X_{C}$. Moreover, since the external friction angle $\delta$ between the wheel and soil is relatively small, it is safe to assume that $\delta \approx 0$. Therefore, the sand bulldozing force model can be simplified further to:

$$
F^{S B}=w \frac{\rho g}{2}\left[\frac{d_{a v g} r_{\alpha}+r_{q}^{2} \tan \phi_{i}}{\cot \left(X_{C}+\phi_{i}\right)}\right]
$$


Finally, using Eq. 5.32 and 5.33, $r_{\alpha}$ can be substitued by $d_{a v g} \cot X_{C}$ and the $r_{q}^{2} \tan \phi_{i}$ term can be substituted by $2 x_{w} d_{a v g}$ :

$$
F^{S B}=w \frac{\rho g d_{a v g}}{2}\left[\frac{d_{a v g} \cot X_{C}+2 x_{w}}{\cot \left(X_{C}+\phi_{i}\right)}\right] .
$$

During the process, this equation of the sand bulldozing force is minimized with respect to $X_{C}$ as the wheel progresses along the $X_{a}$-axis, to find the soil failure angle $X_{c}$. The procedure is explained thoroughly in the next section. Fig. 5.16 provides a recap of the important variables used in the model, showing them from a top and front view of the wheel.
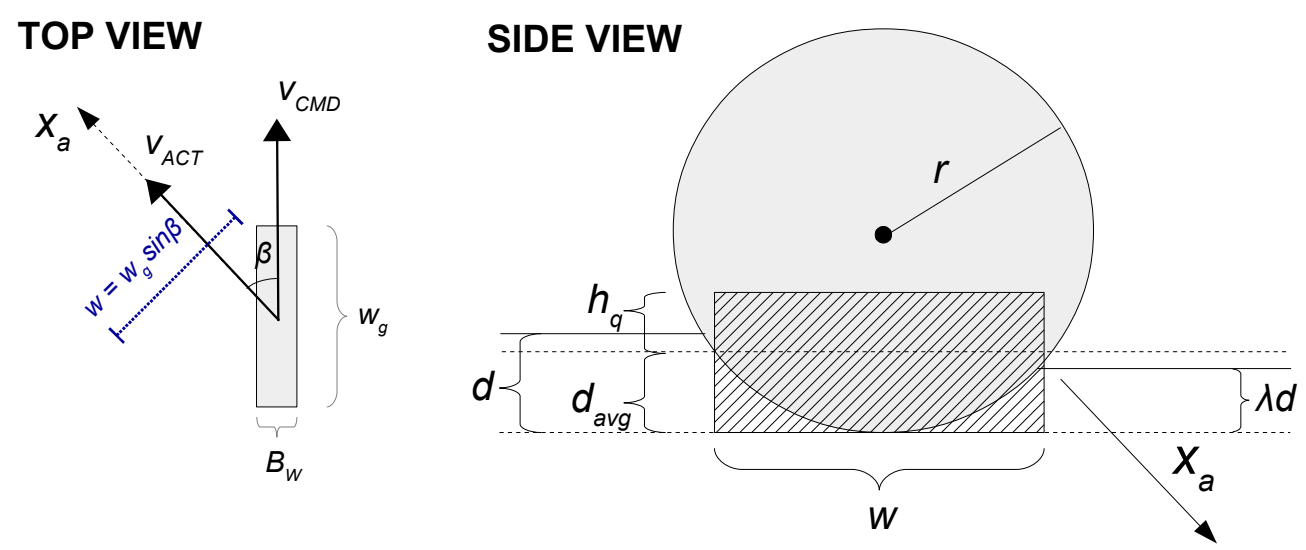

Figure 5.16 Top and front view of a wheel moving along $v^{A C T}$ (or the $X_{a}$-axis).

\section{Procedure}

Before explaining the procedure to compute the inner and outer wheels' sand bulldozing force with respect to time, i.e. $F_{i}^{S B}(t)$ and $F_{o}^{S B}(t)$ respectively, an overall flowchart is presented in Fig. 5.17. This flowchart shows all the parameters required to perform the data post-processing, the sinkage modeling with the $2 \mathrm{D}$ slip-sinkage model, and the sand bulldozing force modeling. The goal is then to have an estimation of the added power with respect to time for the turns tested, namely $P^{A S}(t)$.

Looking at the $F^{S B}$ algorithm block, the inputs to compute the sand bulldozing force for a wheel are the actual velocity $v^{A C T}$, the slip angle $\beta$ and the average sinkage $d_{\text {avg }}$ (with respect to time) of the wheel. This sinkage is used to compute the width of the wheel at the sand level $\left(w_{g}\right)$ and the width of the projected rectangular plate perpendicular to the motion $(w)$ shown in Fig. 5.16 . 


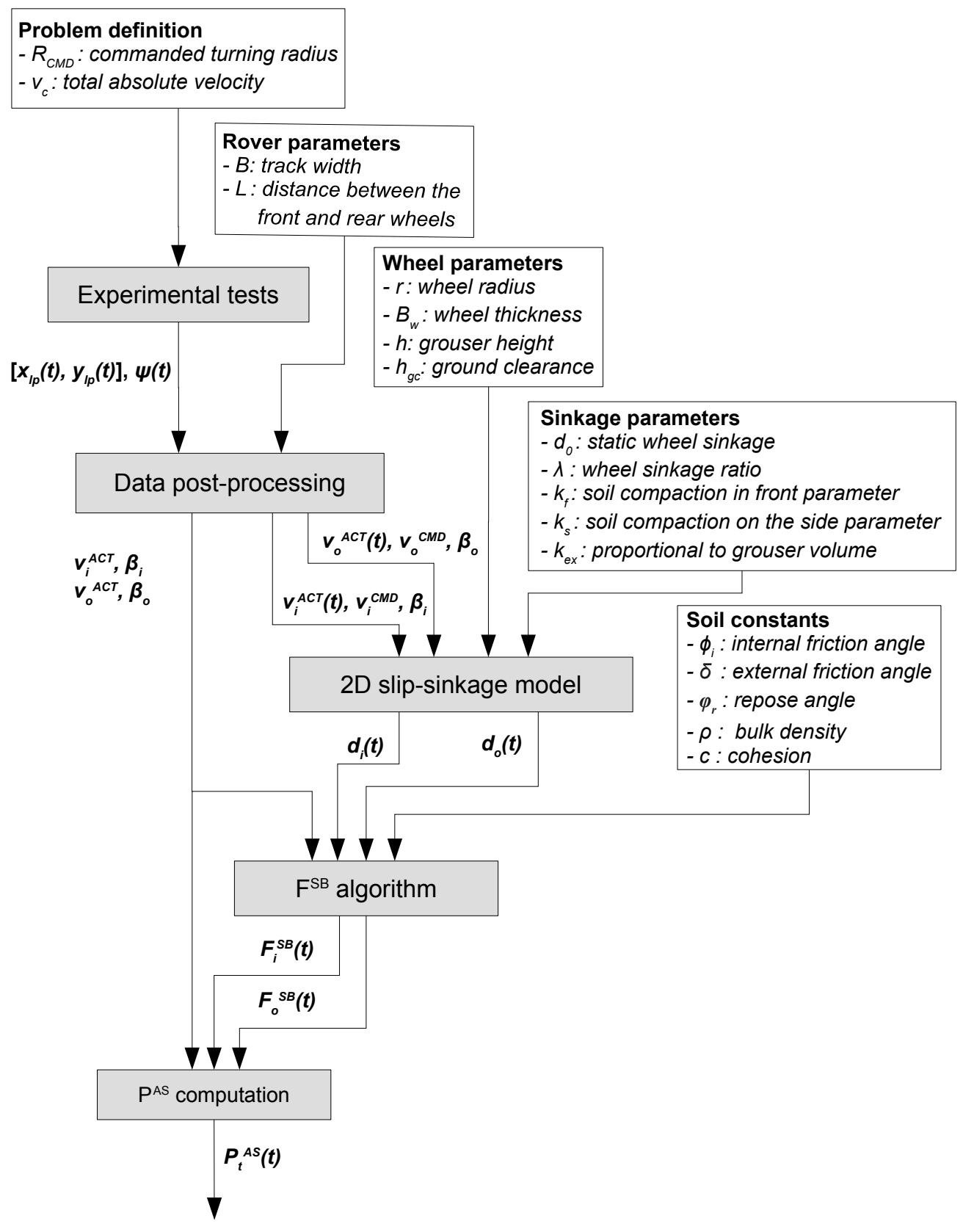

Figure 5.17 Overall flowchart of the proposed solution

The equation to estimate the bulldozing force $F^{S B}$ is a function of the wheel displacement $x_{w}$ (Eq. 5.35). The position of the wheel at each time is first estimated using the average of the actual velocity $v^{A C T}$ and the time interval between the average sinkage values. Then, for each position, this wheel position $x_{w}$, the projected width $w$, and the soil parameters are used in Eq. 5.35 and this function is minimized to find the soil failure angle, i.e. $X_{C}$. Finally, this angle is used to compute the sand bulldozing force using Eq. 5.35. 
To ensure that the bulldozing force reaches a steady state value, the maximum height of the sand pile next to the wheel is estimated using the width of the wheel on the soil level when the sinkage reaches its steady-state value, i.e. $w_{g}^{M A X}$. It is assumed that the sand starts falling off the edges of the sand pile when the angle of the pile reaches the repose angle $\varphi_{r}$. The maximum height of the sand pile can thus be computed following:

$$
h_{q}^{M A X}=w_{g}^{M A X} \tan \varphi_{r}
$$

This maximum sand pile height is used to get the value of $x_{w}$ where the sand pile stops increase and hence finding the maximum sand bulldozing force occurring during the maneuver.

Finally, to get the value of added power that the bulldozing force requires to overcome, the $F^{S B}$ obtained at each time is simply multiplied by the actual wheel velocity $v^{A C T}$ :

$$
P^{A S}=F^{S B} v^{A C T}
$$

For the case of a four-wheeled skid-steered rover, the total sand bulldozing force, $F_{t}^{S B}$, and total added power due to this accumulation of sand, $P_{t}^{A S}$, are computed as

$$
\begin{aligned}
& F_{t}^{S B}=2 F_{i}^{S B}+2 F_{o}^{S B}, \\
& P_{t}^{A S}=2 P_{i}^{A S}+2 P_{o}^{A S},
\end{aligned}
$$

where $P_{i}^{A S}$ and $P_{o}^{A S}$ are the estimation of the required added power to overcome the sand bulldozing forces, $F_{i}^{S B}$ and $F_{o}^{S B}$, of the inner and outer wheels respectively. 


\section{Results}

The sand bulldozing force model was tested with the data gathered from the set of $90^{\circ}$ turns. The measurements were processed as explained in Section 5.1 and 5.3.1 to obtain, for each turn, the actual slip angle $\beta$, the actual velocity $v^{A C T}$, and the average sinkage estimate with respect to time $d_{\text {avg }}(t)$, for the inner and outer wheels. These inputs to the $F^{S B}$ model are shown in Fig. 5.18 as a function of turning radius.
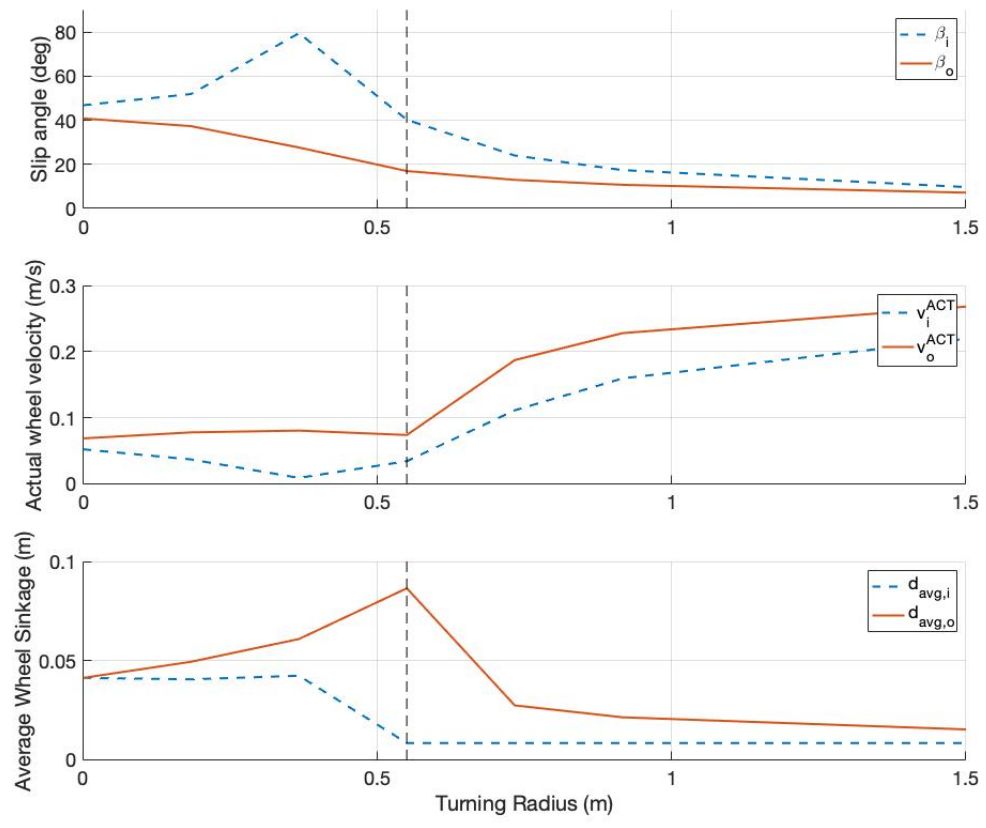

Figure 5.18 Inputs of the sand bulldozing force procedure (coming from the data post-processing and 2D slip-sinkage model).

Fig. 5.19 presents the results of the sand bulldozing foce modeling of the inner and outer wheels, $F_{i}^{S B}$ and $F_{o}^{S B}$ respectively, and the resulting total force for the four wheels. As expected, the force levels off after a few seconds, as the sand pile next to the wheels reach its estimated maximum height. Comparing with the power over time for this set of turns (see Fig. 4.8), it can be observed in both cases that the power and $F_{t}^{S B}$ (total sum of inner and outer bulldozing forces) in the $2 R^{\prime} / 3$ case doesn't seem to stabilize before the end of its $90^{\circ}$ rotation whereas the other turns in the $0 \leq R \leq R^{\prime}$ range reach a steady-state value quite quickly. 

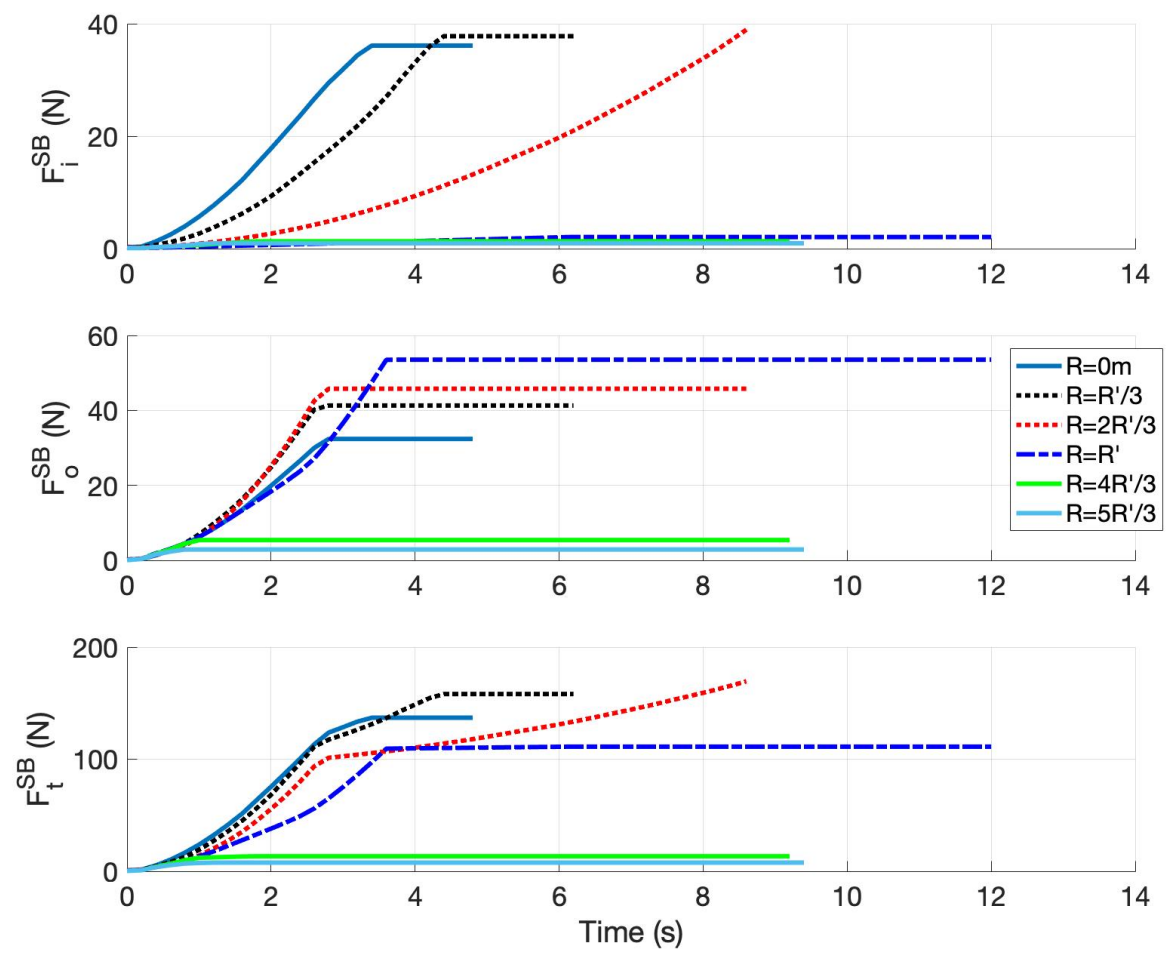

Figure 5.19 Resulting sand bulldozing force $F^{S B}$ for the inner (top) and outer (middle) wheels, as well as the sum of the bulldozing force encountered by the four wheels of the Husky rover (bottom) for the set of turns tested.

Furthermore, the average and final (or steady-state) value of $F_{t}^{S B}$ and $P_{t}^{A S}$ are calculated and plotted against the turning radius of the circular arc path in Fig. 5.20. From the sand bulldozing force results (top plot), it is observed that the trend in measured average power vs. $R$ (Fig. 4.10) is approximately reproduced, especially when looking at the final value of $F_{t}^{S B}$ (dotted line). The bottom plot shows that the distinction between the power required to push the sand pile when $0 \leq R \leq R^{\prime}$ versus when $R>R^{\prime}$ is comparable to Fig. 4.10. However, there is no clear peak of added power in the special case region identified in Section 2.1.2. Also, the $P_{t}^{A S}$ power is relatively small compared to the measured skid-steer rover total power. This suggests that the required added torque to excavate the sand (Section 5.2), particularly in the special case range of turning radii, is a more significant contribution to the increasing power consumption of skid-steer rovers operating on loose soil than bulldozing force is. 

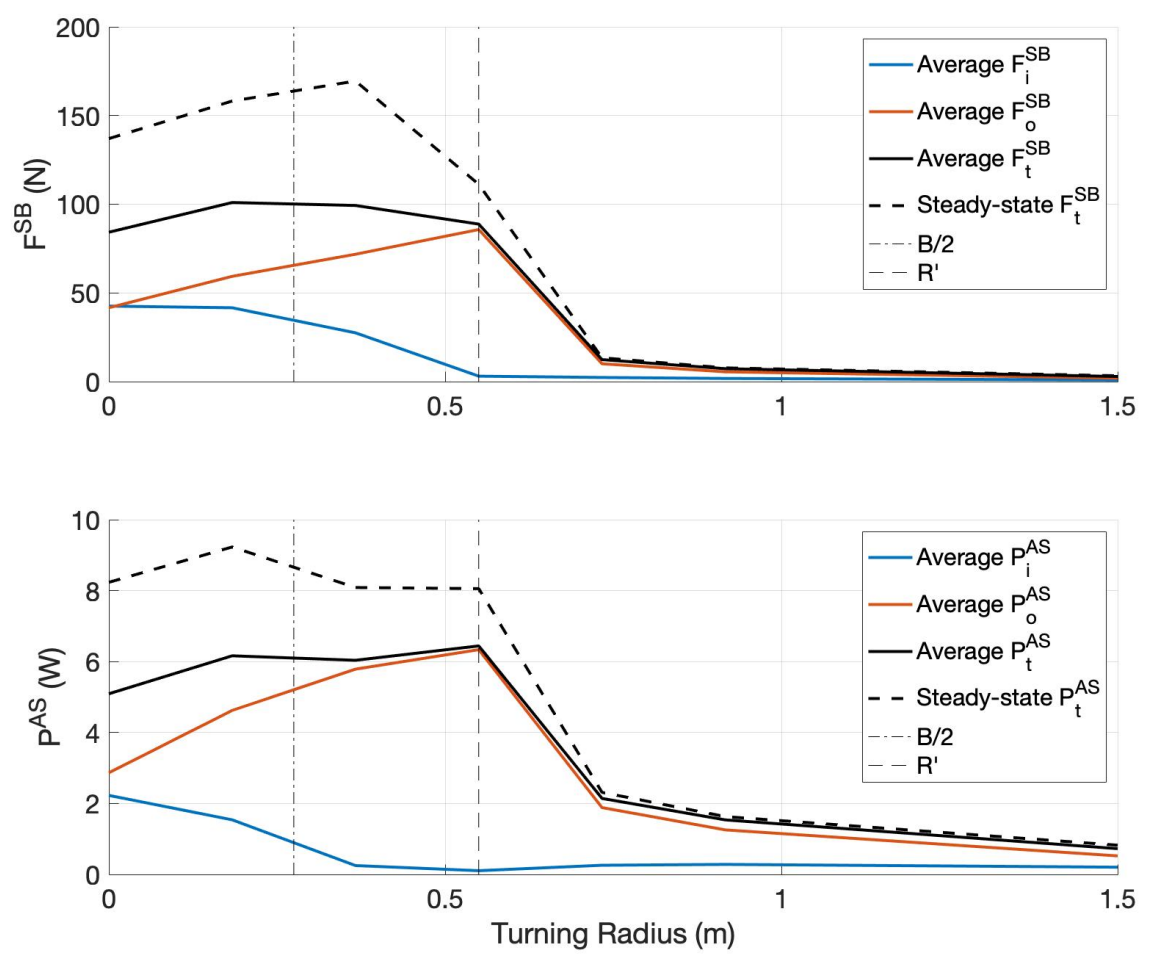

Figure 5.20 Average modeled bulldozing force $F^{S B}$ encountered by the rover during a turn with varying turning radius (top) and corresponding added power $\left(P^{A S}\right)$ required to overcome this force (bottom).

Finally, the modeling of the sand bulldozing force encountered by the wheel of a skid-steer rover while operating on loose soil presented in this section shows some encouraging results but also some key shortcomings. First, the force $F_{t}^{A S}$, and thus also the modeled added total power required to overcome the bulldozing force, i.e. $P_{t}^{A S}$, reproduces the time dependency of the power as it rises as the turn progresses to some extent. Also, the modeled bulldozing force at the end of the turn (top plot of Fig. 5.20) peaks at $2 R^{\prime} / 3$ just like the power at the end of the turn shown in Fig. 4.11. However, this peak is not observed when the sand bulldozing force is converted to $P_{t}^{A S}$ power. Also, this additional power required to overcome the sand resistance is very small relatively to the power consumption of the rover in turns with radius smaller than $R^{\prime}$.

Even if the shortcoming of the model are partially due to shortcomings in the sinkage modeled, it is concluded that, for skid-steer rover operating on loose soil, the impact of the accumulation of sand next to the wheels (creating the sand bulldozing resistance) is a contributing factor to the increasing power consumption, but one that is less significant than the added torque explained in Section 5.2. 


\title{
Chapter 6
}

\section{Application of the Improved Power Model}

\begin{abstract}
An accurate power model for skid-steered wheeled robots on loose soil would allow us to find energy-efficient path for this type of terrain. In this section, two classes of paths are first compared: circle-line-circle (CLC) and point turn-line-poin turn (PLP) paths. The results presented here compare the energy consumed during a CLC path, with a specified turning radius for the two circle segment, namely $R_{C L C}$, against the energy consumed during a corresponding PLP path with corresponding start-to-end displacement and start/end angles. A further analysis of this comparison, for skid-steer rover on hard ground and loose soil, leads to the formulation of angular slip, which has a significant impact on the energy consumption on loose soil.
\end{abstract}




\subsection{CLC vs PLP Paths Comparison}

As shown in Fig. 6.1, this section investigates the energy of CLC and PLP paths when the Clearpath Hucky is operating on flat sand and on flat hard ground. The flat sand experiments were performed at the Mars analogue terrain and the hard ground tests were performed on concrete.

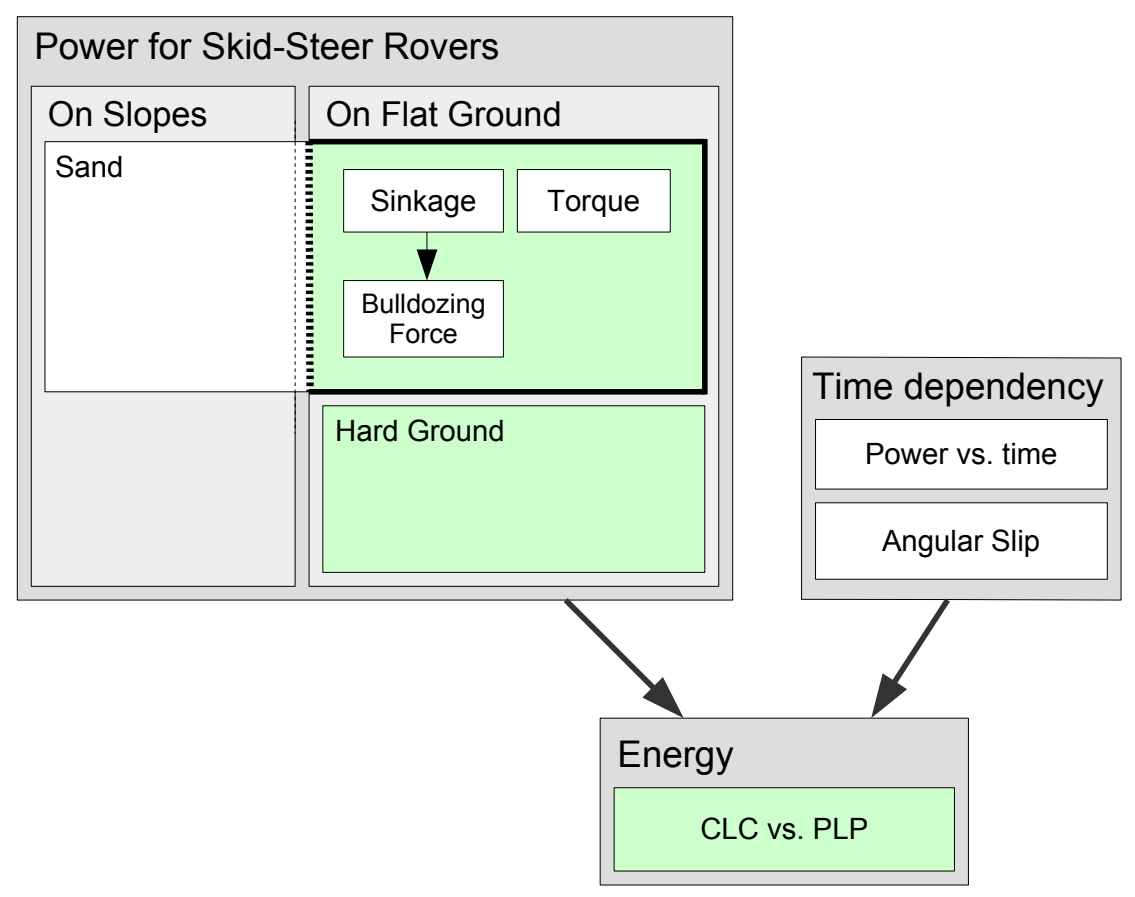

Figure 6.1 High level overview of the topics studied in this research, showing that the circle-linecircle (CLC) and the point turn-line-point turn (PLP) paths were performed both on flat sand and hard concrete terrain.

Since skid-steer rovers often navigate using sets of point turns connected by straight lines, the objective of this work is to show that this may not be the most efficient way to operate. For instance, replacing the point turn segments by wider arc turns could reduce the total energy consumption of the rover. Lower instantaneous power consumption for wider arcs is thus traded off against shorter path distance for tighter arcs to find the most energy-efficient turning radius of the arc path (or circle) segment. Fig. 6.2 depicts a CLC path with the start and end angle $\left(\theta_{\text {start }}\right.$ and $\theta_{\text {end }}$ defined in the path coordinate frame) as well as the length of the straight line segment $\left(d_{\text {straight }}\right)$ connecting the two circle segments of radius $R_{C L C}$. For the corresponding PLP path with the same $\theta_{\text {start }}$ and $\theta_{\text {end }}$ angles, $d_{\text {straight }}$ becomes equal to the start-to-end displacement $d_{p}$. The straight line segment connects two point turns $(R=0)$ of opposite direction, i.e. clockwise first and then counterclockwise. 


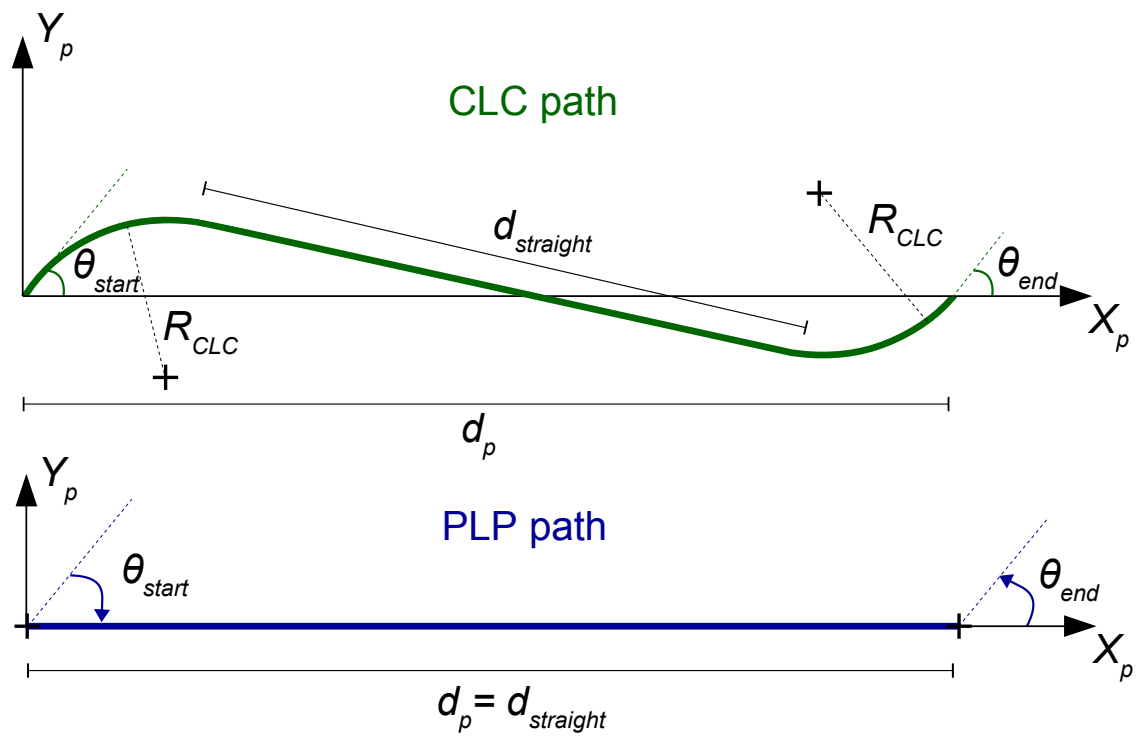

Figure 6.2 Circle-line-circle (CLC) path and its corresponding point turn-line-poin turn (PLP) path in green and blue, respectively. The start and end angles, $\theta_{\text {start }}$ and $\theta_{\text {end }}$ are shown, as well as the length of the straight line segment, $d_{\text {straight }}$, and the start-to-end displacement, $d_{p}$.

Accordingly, a PLP path with $d_{p}=10 \mathrm{~m}$ and $\theta_{\text {start }}=\theta_{\text {end }}=45^{\circ}$ is tested against of a set CLC paths with various turning radii using the Clearpath Husky platform. To have a fair comparison to each of these CLC paths, the PLP path is repeated until the difference between the start-to-end displacement in both cases was within $\pm 2 \%$. After computing the energy consumption of the rover during both paths, the energy difference, $\Delta E \%$, is computed as follow:

$$
\Delta E \%=\frac{E_{C L C}-E_{P L P}}{E_{P L P}}
$$

where $E_{C L C}$ and $E_{P L P}$ are the energy consumption of the CLC and PLP path respectively. A negative energy difference indicates that the CLC path consumed less total energy than its corresponding PLP path. The $\triangle E \%$ results are plotted against $R_{A C T} / R_{A C T}^{\prime}$ where $R_{A C T}$ is the measured turning radius (by circular fit of the position measurement) and $R_{A C T}^{\prime}$ is the measured turning radius when the commanded turning radius is $R^{\prime}$. 
Fig. 6.3a shows that, on hard concrete ground, $R^{\prime}$ is the turning radius where the biggest energy reduction is observed. Concurring research performed a numerical optimization of the energy consumed during a CLC path using the hard ground power model and yielded to the same result, i.e. the optimal turning radius for a CLC path on hard ground is $R^{\prime}$ [34] (see Appendix F).

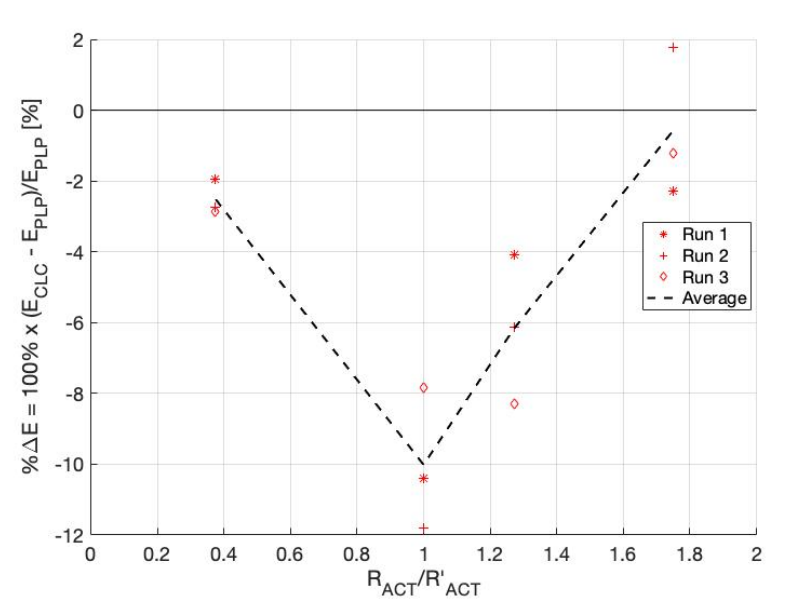

(a)

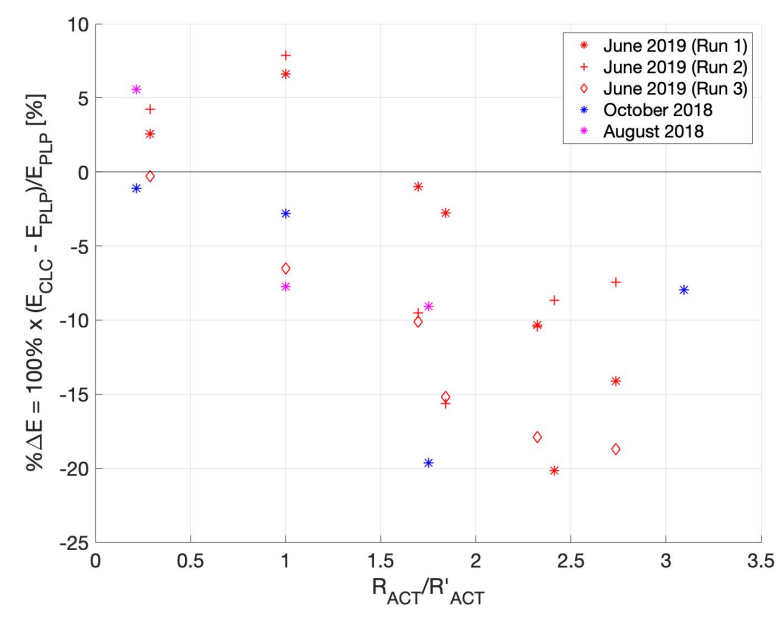

(b)

Figure 6.3 Energy difference percentage $(\triangle E \%)$ as a function of $R_{A C T} / R_{A C T}^{\prime}$ for a $45^{\circ}-10 \mathrm{~m}-45^{\circ}$ path a) on hard concrete ground and b) in the sand of the CSA Mars analogue terrain.

As it can be seen in Fig. 6.3b, the biggest energy reduction on loose soil is observed at around $2<R_{A C T} / R_{A C T}^{\prime}<2.5$. However, in this condition, the energy difference $\Delta E \%$ is quite noisy. This is expected due to the variability of an outdoor sandy terrain. To ensure that the energy reduction (from a PLP to CLC path) observed is not a fluke, a two-tailed t-test [35] is performed to validate that the set of CLC path consumed less energy than the corresponding set of PLP path, i.e. the CLC paths with the same range of start-to-end displacement $d_{p}$ measured.

The first step of the statistical analysis is to select a slice of turning radii $R$ (the treatment variable) to compare with the $R=0$ case (the untreated slice). Then, the start-to-end displacement $d_{p}$ measured (the controlled variable) of the tests in the range of turning radii are used to find the corresponding PLP paths with the same range of displacement measured. The resulting CLC tests are placed in the $\mathbf{O}_{C L C}$ set and the corresponding PLP tests are placed in the $\mathbf{O}_{P L P}$ set. Finally, the energy (the output variable) of the tests contained in the two sets are compared against each other in a two-tailed t-test with the hypothesis that there is a statistical difference between the two. If the $p$-value obtained is lower than 0.05 , then it can be concluded that there is a statistical difference in the energy consumed during a CLC path and its corresponding PLP path. The difference in the mean of the energy consumed during the tests contained in the $\mathbf{O}_{C L C}$ and $\mathbf{O}_{P L P}$ sets then allow us 
to determine if this difference constitute a reduction or an increase in the energy consumed from the PLP to the CLC path.

Fig. 6.4 shows the two resulting sets of tests $\left(\mathbf{O}_{C L C}\right.$ and $\mathbf{O}_{P L P}$ are contained in the green box of Fig. $6.4 \mathrm{a}$ and $6.4 \mathrm{~b}$, respectively) for the selected case of $2<R_{A C T} / R_{A C T}^{\prime}<2.5$. The energy consumption of the tests contained in these two sets are compared in the two-tailed t-test.

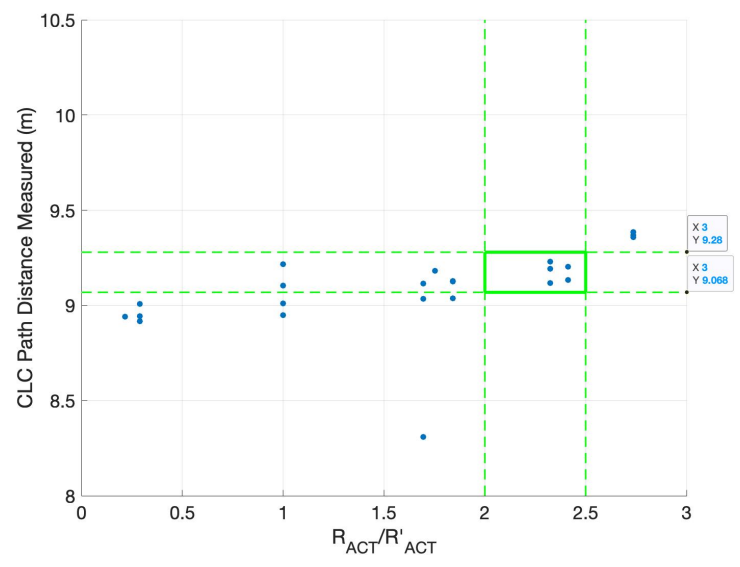

(a)

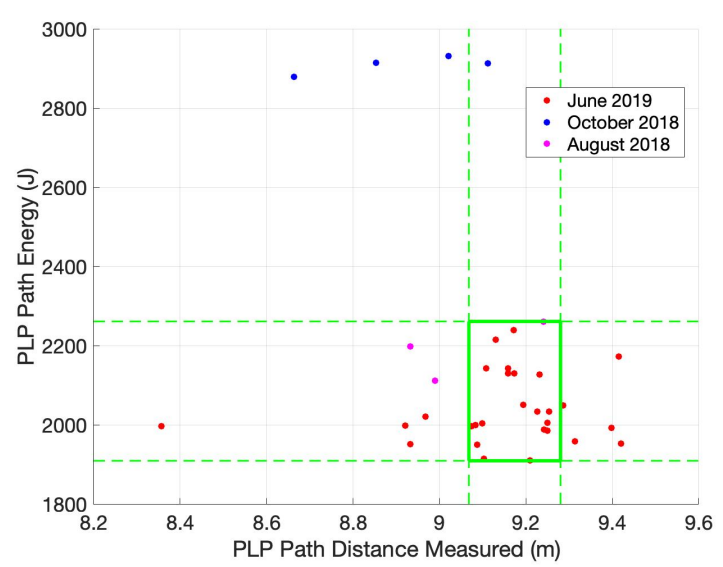

(b)

Figure 6.4 a) CLC path measured distance as a function of $R_{A C T} / R_{A C T}^{\prime}$ showing the range of $d_{p}$ distance for the $\mathbf{O}_{C L C}$ set when $2<R_{A C T} / R_{A C T}^{\prime}<2.5$. b) PLP path energy consumption as a function of $R_{A C T} / R_{A C T}^{\prime}$ showing the corresponding $\mathbf{O}_{P L P}$ set of tests that are in the same range of $d_{p}$ distance as the $\mathbf{O}_{C L C}$ set.

Fig. 6.5 shows the result of the statistical analysis. Only the $0<R_{A C T} / R_{A C T}^{\prime}<0.5$ range didn't yield to a statistical difference between the energy consumption of the CLC and PLP paths. Moreover, the range of turning radii with the greatest confidence in the statistical difference, i.e. the lowest $p$-value, is when $2<R_{A C T} / R_{A C T}^{\prime}<2.5$. As shown in Table 6.1, this was also the set of turns where the biggest energy reduction were observed (by comparing the mean of the CLC and PLP sets). It was also observed that the energy consumed during a CLC path is statistically significantly higher than during a PLP path when $0.5<R_{A C T} / R_{A C T}^{\prime}<1.5$.

It should be noted that the tests gathered during the October 2018 campaign were removed from the analysis as the energy consumed during the PLP path were substantially higher than the two other test campaigns (see Fig. 6.4b). As noted earlier in Section 3.4, weather conditions were wetter during the October 2018 campaign. 

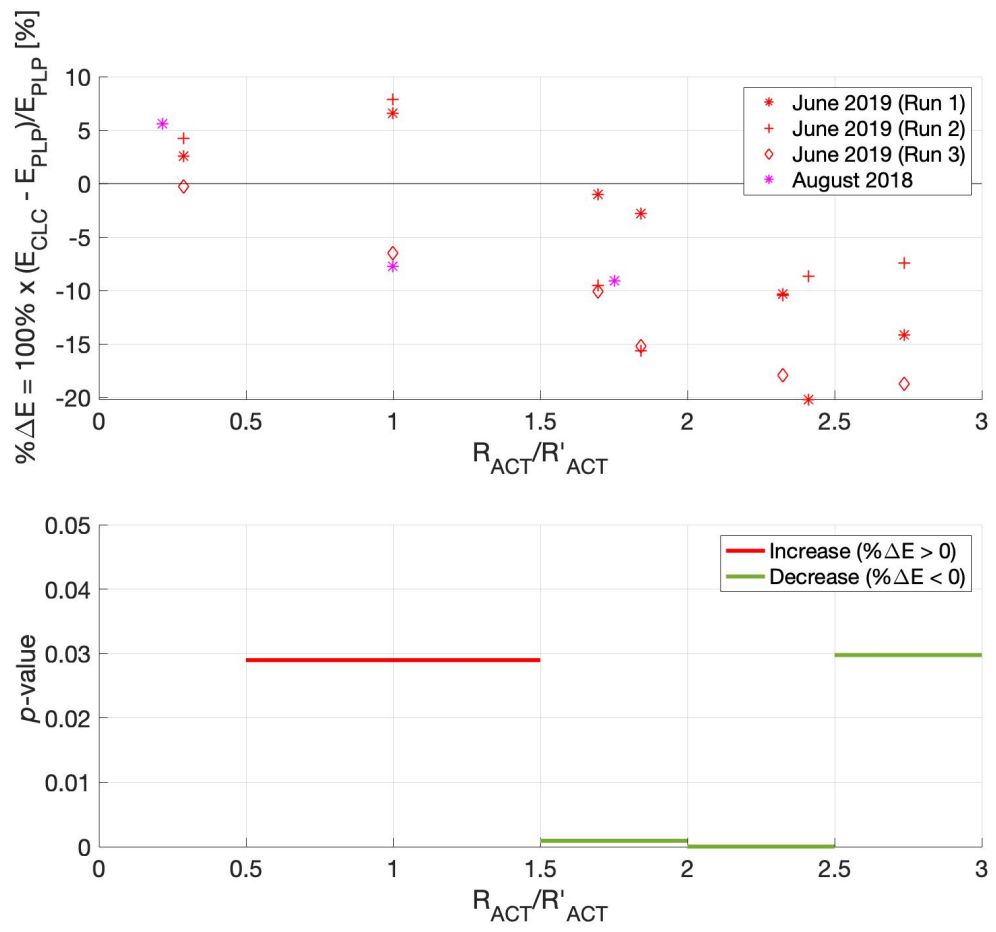

Figure 6.5 $\triangle E \%$ over $R_{A C T} / R_{A C T}^{\prime}$ (top) used for the statistical analysis and obtained $p$-value (bottom) showing if the CLC paths with each set of turning radii has a statistically significant decrease (in green) or increase (in red) of energy consumption compared to a PLP path.

Table 6.1 $p$-value obtained from the statistical analysis using 5 slices of turning radii

\begin{tabular}{lcc}
\hline Turning radius & $p$-value & Difference in mean $\left(E_{C L C}-E_{P L P}\right) / E_{P L P}$ \\
\hline $0<R_{A C T}<0.5 R_{A C T}^{\prime}$ & 0.856 & $+4.55 \%$ \\
$0.5 R_{A C T}^{\prime}<R_{A C T}<1.5 R_{A C T}^{\prime}$ & 0.029 & $+14.38 \%$ \\
$1.5 R_{A C T}^{\prime}<R_{A C T}<2 R_{A C T}^{\prime}$ & $<0.001$ & $-8.63 \%$ \\
$2 R_{A C T}^{\prime}<R_{A C T}<2.5 R_{A C T}^{\prime}$ & $<0.001$ & $\mathbf{- 1 3 . 2 \%}$ \\
$2.5 R_{A C T}^{\prime}<R_{A C T}<3 R_{A C T}^{\prime}$ & 0.03 & $-12.03 \%$ \\
\hline
\end{tabular}

Using the results from the statistical analysis and assuming that the commanded and actual turning radius are equal, i.e. $R_{C L C}=R_{A C T}$ and $R^{\prime}=R_{A C T}^{\prime}$, it can be concluded that the most energyefficient CLC path on loose soil would have a turning radius in the range $2 R^{\prime}<R_{C L C}<2.5 R^{\prime}$, and that it is a statistically significant reduction in the energy consumed versus a PLP path. This path would allow the skid-steer rover to save around $13.2 \%$ of its energy, for a $10 \mathrm{~m}$ path. Other combinations of distance and start/end angles were tested and yielded to similar results, i.e. the turning radius which results in the biggest energy reduction from a PLP to a CLC path occurs at around $2 R^{\prime}<R_{C L C}<2.5 R^{\prime}$ (see Appendix C). 


\subsection{Angular slip}

In order to understand the difference in the results shown in Section 6.1 between hard and loose terrain, the time dependency of power consumption is analyzed in this section. Fig. 6.6 depicts the conditions in which this new concept of angular slip is studied, i.e.on flat hard ground and on flat sand.

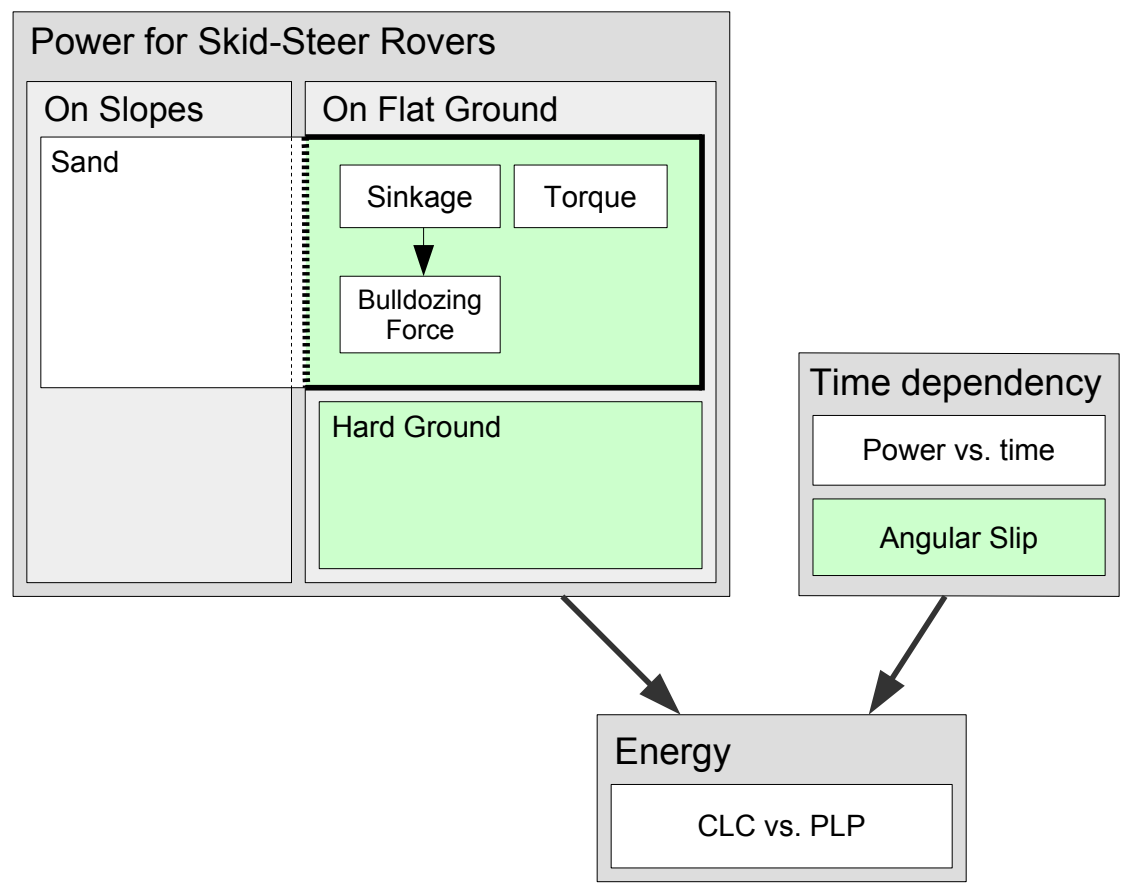

Figure 6.6 High level overview of the topics studied in this research, highlighting that the angular slip is evaluated both on sand and hard ground.

The difference in which turning radius provides the biggest energy reduction between the sand tests and the hard ground tests shown in Fig. 6.3 can partially be explained by the fact that the power model used is not accurate when operating on sand. That being said, it can also be seen in the power vs. $R$ figure (Fig. 4.8) that there isn't a large difference between the average power consumed at $R=0$ and at $R^{\prime}$. Accordingly, another possible contribution to the increased amount of energy consumed during a CLC path with $R^{\prime}$ is that this specific turn takes a longer time to complete its angular rotation. It was shown in Fig. 4.8 that the $R^{\prime}$ turn takes significantly more time to complete its $90^{\circ}$ rotation compared to any other turns. This observation led to calculating the time to complete the $90^{\circ}$ turn for each turning radius. With the constant $v_{c}$ assumption, the commanded angular velocity $\omega_{z}$ is constant below $R^{\prime}$ so the time should be constant for $R \leq R^{\prime}$ (as seen in Fig. 2.1). 
Fig. 6.7 reports the time to complete the same angular change for all the turns on hard ground and in the controlled laboratory sandbox. The results are compared to the expected time (using the commanded angular velocity). This figure clearly illustrates that there is a constant offset between the time to complete the turn on hard ground and that, on a sandy terrain, the offset is significantly bigger (at least double). The difference between the actual and expected time to complete the turn also grows for radii $0 \leq R \leq R^{\prime}$ on sand, peaking at $R^{\prime}$.

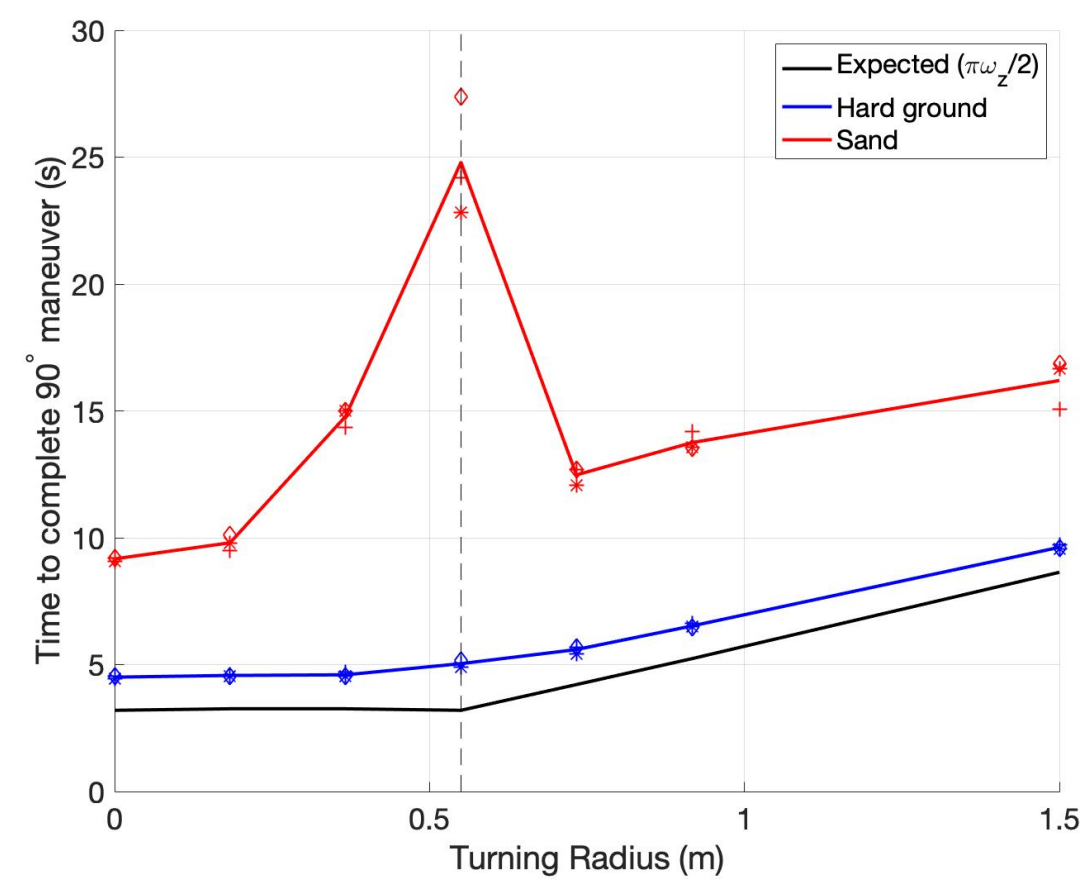

Figure 6.7 Time to complete a $90^{\circ}$ turn of in the laboratory sandbox (in red) and on hard ground (in blue). The expected time to complete the turn is also plotted in black.

As energy is a function of power but also time, the rise in time to complete a turn will increase the total consumed energy. Therefore, this could explain why the greatest energy reduction is not at $R^{\prime}$ when operating on sand (and why, in fact, CLC paths at $R^{\prime}$ on sand consume more energy than PLP).

To quantify the gap between the expected and measured time to complete a turn, it is suggested to use a concept similar to the longitudinal slip (in one direction, along the wheel axis). This longitudinal slip $s_{y}$ along the rover's $y$-axis is defined as

$$
s_{y}=\frac{v^{C M D}-v_{y}^{A C T}}{v^{C M D}},
$$


where $v^{C M D}$ is the wheel commanded linear velocity and $v_{y}^{A C T}$ is the actual (or measured) velocity along the rover's $y$-axis. Accordingly, the angular slip can similarly be defined as

$$
s_{\omega}=\frac{\omega_{z}^{C M D}-\omega_{z}^{A C T}}{\omega_{z}^{C M D}},
$$

where $\omega_{z}^{C M D}$ is the commanded angular velocity and $\omega_{z}^{A C T}$ is the actual (or measured) angular velocity. The commanded and average actual angular velocities for the set of turns tested are shown in Fig. 6.8. This figure also reports the computed angular slip $s_{\omega}$ for each turning radius. As expected, the angular slip is greater for all the turning on sandy terrain and the peak at $R^{\prime}$ is also captured.

An estimation of this angular slip as a function of turning radius could be useful when computing the energy that a skid-steer rover needs to consume during a trajectory. Accordingly, the optimal turning radius for a CLC path on sand could be predicted accurately as there is multiple parameters that need to be traded off such as: the length of the path (which increases with $R$ ), the time to complete a turn, and the instantaneous power consumption.
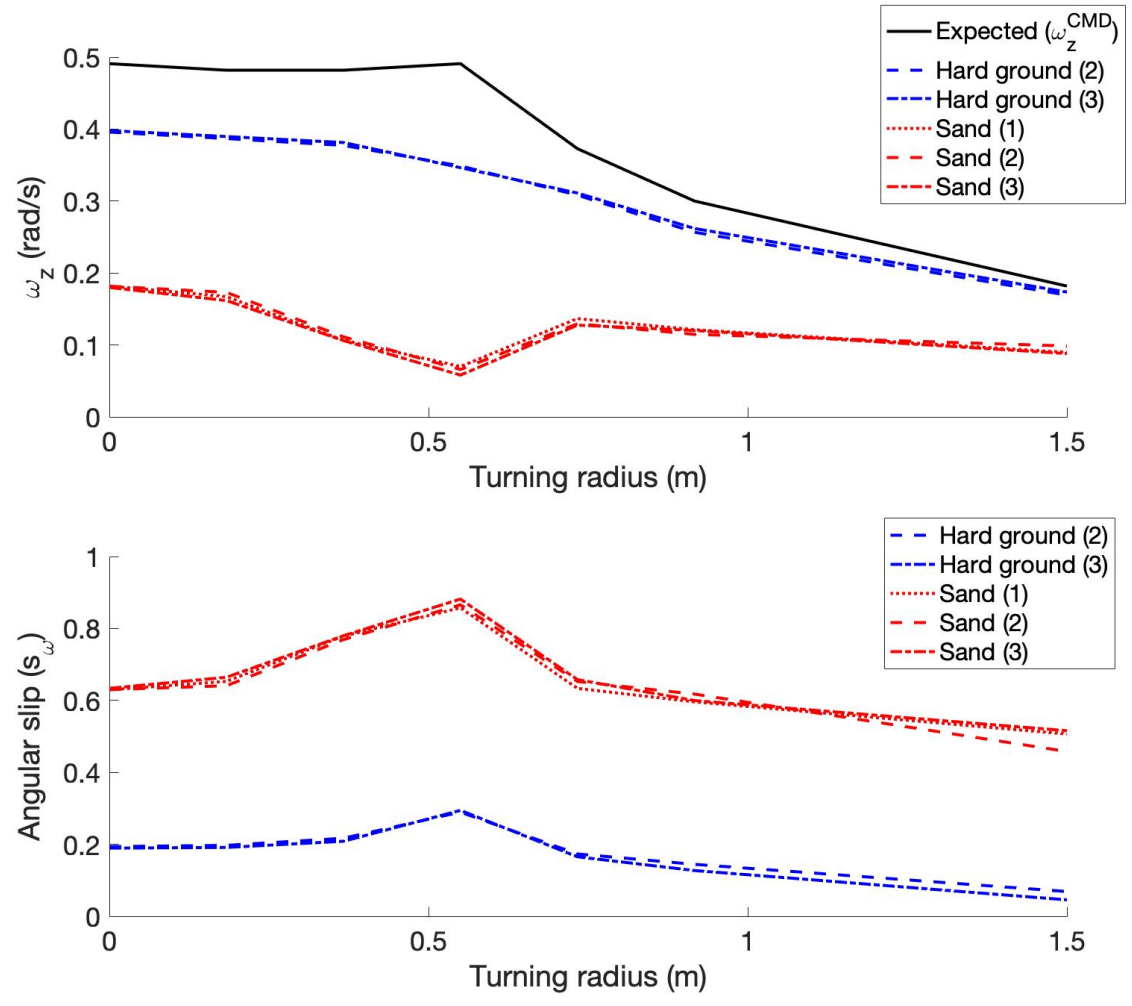

Figure 6.8 Actual and commanded angular velocity $\omega_{z}$ as a function of turning radius (top) and the resulting angular slip $s_{\omega}$ (bottom) for hard ground and sand. 
Fig. 6.9 shows that the angular velocity quickly decreased as the turn progress. An interesting phenomenon is also observed: the angular velocity seems to increase again for all the turning radii except for $2 R^{\prime} / 3$, which is the only turning radius in the special case region $\left(B / 2<R<R^{\prime}\right)$.

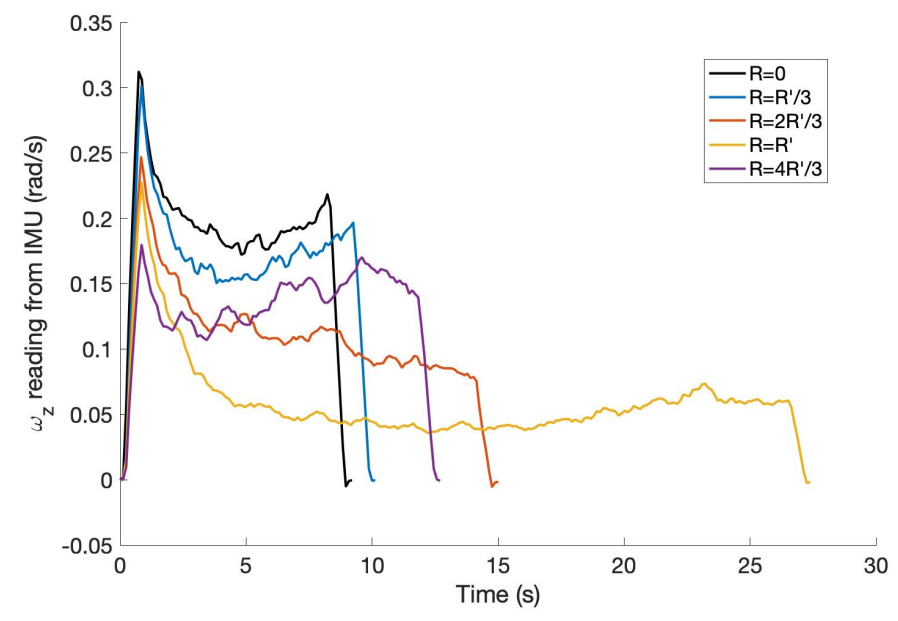

Figure 6.9 Angular velocity $\left(\omega_{z}\right)$ over time for the turning radii tested on sand.

To explain the increase in angular slip at $R^{\prime}$, the forces recorded during the set of circular arc path with the single-wheel test bed (shown in Section 3.3) were analyzed. The $F_{x}$ and $F_{z}$ forces measured by the force/torque sensor are first converted to tangential and radial forces, i.e. $F_{\text {tang }}$ and $F_{\text {radial }}$ respectively, using the equations shown in Fig. 6.10a. Fig 6.10b presents the $F_{\text {tang }}$ and

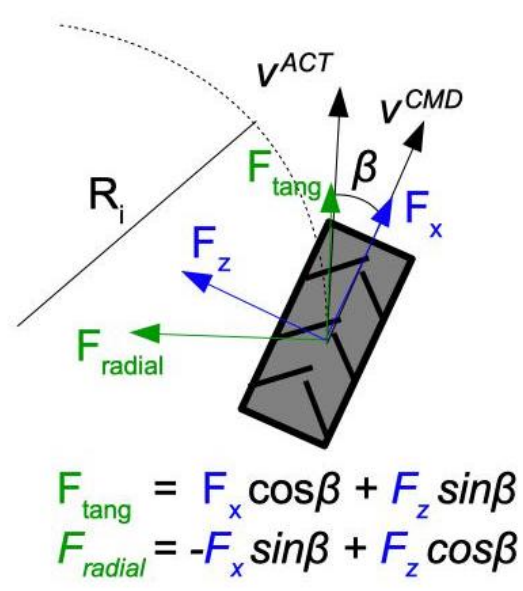

(a)
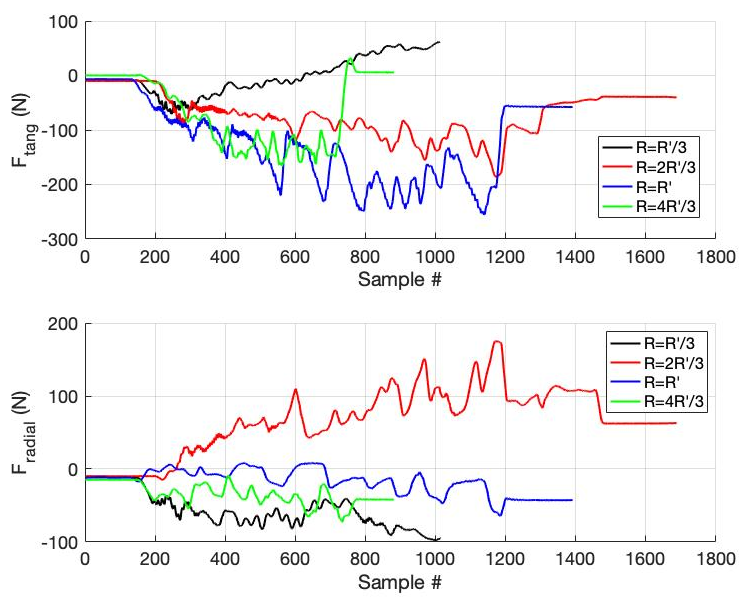

(b)

Figure 6.10 a) Schematic figure showing the orientation of the forces measured by the force/torque sensor (in blue) and how these forces were converted to $F_{\text {tang }}$ and $F_{\text {radial }}$ b) Resulting tangential and radial forces as the wheel progresses on its circular arc path, for the four turns tested. 
$F_{\text {radial }}$ forces as the wheel turn progresses during the four turns tested. Looking at the tangential force, it can be noticed that the highest amount of resistance is recorded at $R^{\prime}$, which could explain why this particular turn takes more time than expected to complete its rotation, i.e. it has a higher angular slip.

It was demonstrated by previous work [36] that a braked wheel produces a much different flow pattern in the soil than a rolling wheel. Using visual analysis, it was shown that pushing a braked wheel generates soil displacement only in the sliding direction, resulting in a large shearing surface in the soil. On the other hand, a rolling wheel produces more vertical soil motion and a smaller shear plane.

In the case of a $R^{\prime}$ turn, the inner wheels are not commanded to rotate which is analogous to pushing a braked wheel. This could be the cause of the increase in the resistance force (opposite to the direction of $F_{\text {tang }}$ ) for the $R^{\prime}$ turn. To investigate even further, the single-wheel test bed was used to move a wheel along a linear path perpendicular to its rotation direction, i.e. $\beta=90^{\circ}$. The commanded linear velocity of the wheel was set to $0,0.02$ and $0.04 \mathrm{~m} / \mathrm{s}$ and the resulting force in the $F_{z}$ direction is shown in Fig. 6.11b. It is clear that the braked wheel (when $v^{C M D}=0 \mathrm{~m} / \mathrm{s}$ ) produces larger sand resistance force than a rolling wheel following the same trajectory.

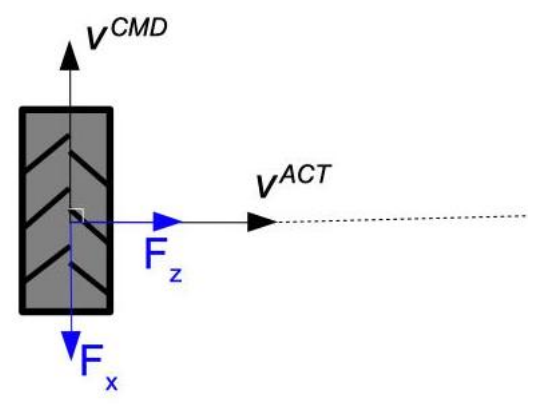

(a)

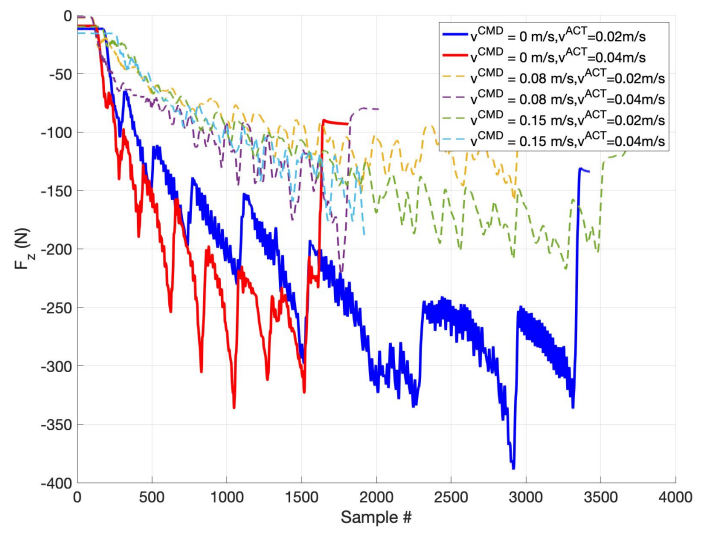

(b)

Figure 6.11 a) Schematic figure of a linear path test with $\beta=90^{\circ}$ showing the orientation of the forces measured. b) Corresponding $F_{z}$ measured of combinations of 3 commanded wheel velocities and 2 actual velocites. The $F_{z}$ force of the braked wheel when $v^{A C T}=0.02 \mathrm{~m} / \mathrm{s}$ and $v^{A C T}=0.04$ $\mathrm{m} / \mathrm{s}$ are shown as blue and red solid lines, respectively.

To conclude, as the resistance force parallel to the linear motion (or tangential to the arc motion) increases, a decrease in the measured angular velocity is observed (Fig. 6.9) and hence resulting in an increase of average angular slip during the turn (Fig. 6.8). 


\section{Chapter 7}

\section{Conclusion and Future Work}

This section summarizes the main conclusions of this research and presents the recommendations and future research work. Several of the conclusions relate to a key turning radius, identified as particularly important; $R^{\prime}$, which is defined as the turning radius where the inner wheels are not commanded to rotate.

\section{Power Consumption of Skid-Steer Rovers on Loose Soil}

A key contribution of this work is the identification of a special case in the power consumption of skid-steered wheeled robots on sand. When the turning radius is slightly bigger than half of the width of the rover but smaller than $R^{\prime}$, i.e. $B / 2<R<R^{\prime}$, measured power consumption is significantly higher than existing models predict. As shown in Section 4.4.2, this special case has an average power consumption approximately $20 \%$ higher than for a point turn, and it exhibits the highest rise in instantaneous power with respect to time. These results were observed both in the controlled laboratory sandbox filled with GRC-1 simulant and in the field at the Mars analogue terrain.

Accordingly, contributing factors to explain the increasing power consumption of this special case have been identified. The added motor torque required to rotate the inner wheels backwards as they are being dragged forward by the outer wheels contributes to the power peak for the set of turning radii in the special case range. Here, the wheels must pass through piles of sand they have excavated. It was also observed that this counter-rotation causes the inner wheels to sink more as the steady-state sinkage also peaks for the special case turns. This additional sinkage results in a higher bulldozing force required to push the sand accumulated next to the wheels, although this contribution is less significant than the added torque described above.

Another important phenomenon reported in Section 4.4.1 is the fact that the power consumption of a skid-steered wheeled vehicle rises as the turn progresses for turns of radii $R \leq R^{\prime}$. The highest increase during the $90^{\circ}$ turns tested occurred at $R=2 R^{\prime} / 3$, which correspond to the turning radius 
in the special case region, i.e. $B / 2<2 R^{\prime} / 3<R^{\prime}$.

\section{Neglecting Roll in Skid-Steer Rover Power Model}

As shown in Section 4.1, plotting the power consumption of a skid-steer rover as a function of vehicle's pitch and roll validated that the roll of the robot can be neglected when modelling the power consumption of a sloped terrain composed of loose soil. Although the existing hard ground power model from the literature also ignored the influence of roll on power consumption, this work also validated that this was true on sandy slopes using experimental data from the White Sands National Monument test campaign. This contribution will be useful in the development of a power model for skid-steered wheeled robot operating on loose soil with slopes.

\section{Power Consumption with Respect to Time and Energy-Efficient Paths}

Finding energy-efficient paths is an ultimate goal for studying rover power consumption. Since energy is the integral of power over time, we also studied the time dependency aspect of a skid-steer rover on loose soil. Accordingly, the power over time figures presented in Section 4.4.1 exposed the fact that turns with a turning radius $R \leq R^{\prime}$ take a longer time to complete their angular rotation as $R$ increases. This conclusion is only true for sandy soil as hard ground experiments confirmed that the angular velocity (or time to complete the desired rotation) is constant for turns below $R^{\prime}$, under the constant $v_{c}$ velocity constraint. In other words, the measured (or actual) angular velocity is closer to the commanded angular velocity on hard ground. The concept of angular slip is introduced in this work to describe the difference between actual and commanded angular velocity. Results show that the angular slip is significantly higher on sand than on hard ground and peaks at $R^{\prime}$ to a value $40 \%$ higher than when $R=0$.

This conclusion was used to interpret the energy reduction from the two types of path presented in Section 6.1. The total energy of a point turn-line-point turn (PLP) path was compared to the total energy of a circle-line-circle (CLC) both on hard ground and on loose terrain. On hard ground, the turning radius where the highest reduction (from PLP to CLC) was observed was $R^{\prime}$ for the two paths tested, which coincides with the theoretical analysis. On the other hand, on loose terrain, the turning radius where the highest energy reduction was observed was around $R_{C L C}=2.5 R^{\prime}$. As the CLC path with $R_{C L C}=R^{\prime}$ takes more time to complete its rotation due to angular slip, there is a trade-off occurring between the length of the path (which increases with $R_{C L C}$ ), the angular slip and the average power consumption of the skid-steer rover during a turn. 


\section{Future Work and Practical Lessons Learned}

Towards having a power model specific to skid-steer rovers operating on loose soil, future work will first be to model terramechanics of the soil shear failure when the wheel is excavating a growing pile of sand. As this occurs for the inner wheels when the rover is performing turn in the special range of turning radii identified, i.e. $B / 2<R<R^{\prime}$, an estimation of the amount of torque required for the wheels to excavate and compact the sand would benefit the overall power consumption prediction. Although it was shown that the sand bulldozing force that rover needs to overcome while skidding is a less important contributing factor to the increased power consumption below $R^{\prime}$, improvements should be made on the novel 2D slip-sinkage presented in this work. Amongst other, additional work to improve the model is to consider the added sinkage in the special case region and when the inner wheels aren't commanded to turn (at $R^{\prime}$ ). Successfully predicting the sinkage would not only improve the predicted power required to overcome the sand bulldozing force but also help understand the behavior of skid-steered wheeled robots on loose soil. It would also be interesting to study the effects of the wheel design on the sinkage but also on the added excavation torque aforementioned. These two factors would need to be traded off when considering the thickness on the wheel, for example. A thicker wheel, i.e a larger $B_{w}$, would sink less as the load is distributed on a larger contact area, but would excavate more sand in front (or back) due to this larger contact area. Additionaly, a hollow wheel could reduce the amount of bulldozing resistance force encountered by the rover while skidding during a turn.

Once the added torque is modeled and the improvements to the $2 \mathrm{D}$ slip-sinkage model are applied, the idea is to have an online estimation of the power model key parameters as the skidsteer rover is operating on sand. These parameters would therefore be updated in situ using the instantaneous power measured, prior to using the power model to find energy-efficient paths.

Furthermore, it would be interesting to investigate on the new concept of angular slip presented in this thesis. As mentioned, a prediction of this slip is important when finding energy-efficient paths as the total energy consumed during a trajectory is considerably affected by the time to complete the desired maneuvers.

Finally, even before a power model is developed to fully account for the two contributing factors of increased power listed above, a practical lesson learned is to avoid turns of radii $B / 2<R<R^{\prime}$ whenever possible when designing an energy-efficient path for skid-steered wheeled vehicles on loose soil. In fact, experimental results demonstrated that the most energy-efficient turning radius for a path composed of a circular arc, a straight line and another circular segment, i.e. CLC path, was around $2-2.5 R^{\prime}$ on sand, and at $R^{\prime}$ on hard concrete ground. 


\section{Bibliography}

[1] J. L. Martínez, A. Mandow, J. Morales, S. Pedraza, and A. García-Cerezo, "Approximating kinematics for tracked mobile robots", The International Journal of Robotics Research, vol. 24, no. 10, pp. 867-878, 2005.

[2] A. Mandow, J. L. Martinez, J. Morales, J. L. Blanco, A. Garcia-Cerezo, and J. Gonzalez, "Experimental kinematics for wheeled skid-steer mobile robots", in 2007 IEEE/RSJ International Conference on Intelligent Robots and Systems, IEEE, 2007, pp. 1222-1227.

[3] G. Reina and R. Galati, "Slip-based terrain estimation with a skid-steer vehicle", Vehicle System Dynamics, vol. 54, no. 10, pp. 1384-1404, 2016.

[4] J. Pentzer, S. Brennan, and K. Reichard, "Model-based prediction of skid-steer robot kinematics using online estimation of track instantaneous centers of rotation”, Journal of Field Robotics, vol. 31, no. 3, pp. 455-476, 2014.

[5] J. Morales, J. L. Martinez, A. Mandow, A. J. García-Cerezo, and S. Pedraza, "Power consumption modeling of skid-steer tracked mobile robots on rigid terrain", IEEE Transactions on Robotics, vol. 25, no. 5, pp. 1098-1108, 2009.

[6] J. Pentzer, S. Brennan, and K. Reichard, "On-line estimation of vehicle motion and power model parameters for skid-steer robot energy use prediction", in 2014 American Control Conference, IEEE, 2014, pp. 2786-2791.

[7] J. Pentzer, K. Reichard, and S. Brennan, "Energy-based path planning for skid-steer vehicles operating in areas with mixed surface types", in 2016 American Control Conference (ACC), IEEE, 2016, pp. 2110-2115.

[8] W. Yu, O. Y. Chuy Jr, E. G. Collins Jr, and P. Hollis, "Analysis and experimental verification for dynamic modeling of a skid-steered wheeled vehicle", IEEE transactions on robotics, vol. 26, no. 2, pp. 340-353, 2010.

[9] J. Y. Wong, Theory of ground vehicles. John Wiley \& Sons, 2008. 
[10] C. Ordonez, N. Gupta, W. Yu, O. Chuy, and E. G. Collins, "Modeling of skid-steered wheeled robotic vehicles on sloped terrains", in ASME 2012 5Th Annual Dynamic Systems and Control Conference Joint with the JSME 2012 11Th Motion and Vibration Conference, American Society of Mechanical Engineers, 2012, pp. 91-99.

[11] N. Gupta, C. Ordonez, and E. G. Collins, "Dynamically feasible, energy efficient motion planning for skid-steered vehicles”, Autonomous Robots, vol. 41, no. 2, pp. 453-471, 2017.

[12] W. Yu, E. Collins, and O. Chuy, "Dynamic modeling and power modeling of robotic skidsteered wheeled vehicles", Mobile Robots-Current Trends, pp. 291-318, 2011.

[13] S. Dogru and L. Marques, "A physics-based power model for skid-steered wheeled mobile robots", IEEE Transactions on Robotics, vol. 34, no. 2, pp. 421-433, 2018.

[14] G. Ishigami, A. Miwa, K. Nagatani, and K. Yoshida, "Terramechanics-based model for steering maneuver of planetary exploration rovers on loose soil", Journal of Field Robotics, vol. 24, no. 3, 233-250, 2007.

[15] B. Shamah, "Experimental comparison of skid steering vs. explicit steering for wheeled mobile robot," m. sc", 1999.

[16] M. Heverly, J. Matthews, J. Lin, D. Fuller, M. Maimone, J. Biesiadecki, and J. Leichty, "Traverse performance characterization for the mars science laboratory rover", Journal of Field Robotics, vol. 30, no. 6, pp. 835-846, 2013.

[17] C. Cunningham, M. Ono, I. Nesnas, J. Yen, and W. L. Whittaker, "Locally-adaptive slip prediction for planetary rovers using gaussian processes", in 2017 IEEE International Conference on Robotics and Automation (ICRA), IEEE, 2017, pp. 5487-5494.

[18] M. G. Bekker, "Introduction to terrain-vehicle systems. part i: The terrain. part ii: The vehicle”, MICHIGAN UNIV ANN ARBOR, Tech. Rep., 1969.

[19] M. Lyasko, "Slip sinkage effect in soil-vehicle mechanics", Journal of Terramechanics, vol. 47, no. 1, pp. 21-31, 2010.

[20] A. R. Reece, "Problems of soil vehicle mechanics", ARMY TANK-AUTOMOTIVE CENTER WARREN MI, Tech. Rep., 1964.

[21] A. Vasil'ev, E. Dokychaeva, and O. Utkin-Lubovtsov, "Effect of tracked tractor design parameters on tractive performance", Moscow: Mashinostroenie, 1969.

[22] L. Ding, H.-b. Gao, Z.-q. Deng, and J.-g. Tao, "Wheel slip-sinkage and its prediction model of lunar rover", Journal of Central South University of Technology, vol. 17, no. 1, pp. 129$135,2010$. 
[23] J. Y. Wong and A. Reece, "Prediction of rigid wheel performance based on the analysis of soil-wheel stresses part i. performance of driven rigid wheels", Journal of Terramechanics, vol. 4, no. 1, pp. 81-98, 1967.

[24] Z. Janosi, "The analytical determination of drawbar pull as a function of slip for tracked vehicles in defarmable soils", in Proc. of 1st Int. Conf. of ISTVS. Turin., 1961, 1961.

[25] R. Irani, R. Bauer, and A Warkentin, “A dynamic terramechanic model for small lightweight vehicles with rigid wheels and grousers operating in sandy soil", Journal of Terramechanics, vol. 48, no. 4, pp. 307-318, 2011.

[26] M. G. Bekker, "Off-the-road locomotion", Research and development in terramechanics, 1960.

[27] E. Hegedus, A simplified method for the determination of bulldozing resistance. US Army Ordnance Tank-Automotive Command, 1960.

[28] K. Skonieczny, "Modeling the effects of surcharge accumulation on terrestrial and planetary wide-blade soil-tillage tool interactions", Soil and Tillage Research, vol. 176, pp. 104-111, 2018.

[29] H. Oravec, X Zeng, and V. Asnani, "Design and characterization of grc-1: A soil for lunar terramechanics testing in earth-ambient conditions", Journal of Terramechanics, vol. 47, no. 6, pp. 361-377, 2010.

[30] C. Creager, V. Asnani, H. Oravec, and A. Woodward, "Drawbar pull (dp) procedures for off-road vehicle testing", 2017.

[31] A. C. Woodward, "Experimental analysis of the effects of the variation of drawbar pull test parameters for exploration vehicles on grc-1 lunar soil simulant”, PhD thesis, Virginia Tech, 2011.

[32] C. M. Creager, L. Jones, and L. M. Smith, "Effect of angle of attack on slope climbing performance", 2017.

[33] H. Inotsume, C. Creager, D. Wettergreen, and W. Whittaker, "Finding routes for efficient and successful slope ascent for exploration rovers", in The International Symposium on Artificial Intelligence, Robotics and Automation in Space (i-SAIRAS), 2016.

[34] M. Effati, J.-S. Fiset, and K. Skonieczny, "Considering slip-track for energy-efficient paths of skid-steer rovers", Submitted, 2019.

[35] G. A. Simon and J. F. Freund, Modern elementary statistics. Prentice-Hall International, 1997. 
[36] K. Skonieczny, S. J. Moreland, V. M. Asnani, C. M. Creager, H. Inotsume, and D. S. Wettergreen, "Visualizing and analyzing machine-soil interactions using computer vision", Journal of Field Robotics, vol. 31, no. 5, pp. 820-836, 2014. 


\section{Appendix A}

\section{Derivation of equations for $v_{y}, \omega_{z}, V_{l}$ and $V_{r}$ based on $v_{c}$ and $R$ for left turns $\left(V_{r} \geq V_{l}\right)$}

Using the $R^{\prime}$ definition and Eq. 2.4, the $V_{r}$ velocity is first isolated.

$$
\begin{gathered}
R=R^{\prime}\left(\frac{V_{r}+V_{l}}{V_{r}-V_{l}}\right) \\
R\left(V_{r}-V_{l}\right)=R^{\prime}\left(V_{r}+V_{l}\right) \\
V_{r}\left(R-R^{\prime}\right)=V_{l}\left(R+R^{\prime}\right) \\
V_{r}=\left(\frac{R+R^{\prime}}{R-R^{\prime}}\right) V_{l}
\end{gathered}
$$

Using the $v_{c}$ defined in Eq. 2.6, the left and right linear velocities can be evaluated in the following three cases.

Case $1: R<R^{\prime}$

In this region, we have $V_{l}<0$ and $V_{r}>0$ such that

$$
2 v_{c}=V_{r}-V_{l}
$$

Therefore, we use Eq A.4 to find that 


$$
\begin{gathered}
V_{l}=\frac{2 v_{c}}{\frac{R+R^{\prime}}{R-R^{\prime}}-1} \\
V_{r}=2 v_{c}+V_{l}
\end{gathered}
$$

Case $2: R=R^{\prime}$

By definition, at $R^{\prime}$, we have that

$$
\begin{gathered}
V_{l}=0 \\
V_{r}=2 v_{c} .
\end{gathered}
$$

Case $3: R>R^{\prime}$

In this region, we have $V_{l}>0$ and $V_{r}>0$ such that

$$
2 v_{c}=V_{r}+V_{l}
$$

Therefore, we use Eq A.4 to find that

$$
\begin{gathered}
V_{l}=\frac{2 v_{c}}{\frac{R+R^{\prime}}{R-R^{\prime}}+1} \\
V_{r}=2 v_{c}-V_{l}
\end{gathered}
$$

Solution To summarize, we obtain the following solution for left and right velocities:

$$
V_{l}=\left\{\begin{array}{ll}
\frac{2 v_{c}}{R+R^{\prime}}, & \text { if } R<R^{\prime} \\
0, & \text { if } R=R^{\prime} \\
\frac{2 v_{c}}{R+R^{\prime}}, & \text { if } R>R^{\prime} \\
\frac{R-R^{\prime}}{R}+1 & (\mathrm{~A} .13)
\end{array} \quad V_{r}= \begin{cases}2 v_{c}+V_{l}, & \text { if } R<R^{\prime} \\
2 v_{c}, & \text { if } R=R^{\prime} \\
2 v_{c}-V_{l}, & \text { if } R>R^{\prime}\end{cases}\right.
$$




\section{Appendix B}

\section{Other Single-Wheel Test Bed Results}

In this section, additional results from the test performed with the Husky wheel mounted on the five-axis single-wheel test bed are shown. As explained in Section 5.3.2, linear path were performed with a specified slip angle $\beta$, commanded wheel velocity $v^{C M D}$, and actual wheel displacement velocity $v^{A C T}$. Fig. B.1 shows the relative sinkage results for two different actual velocities and the $100 \%$ slip case (where $v^{A C T}=0 \mathrm{~m} / \mathrm{s}$ ). It should be noticed that the test case with $\beta>90^{\circ}$, which occurs in the special range of turning radii indentified (when $B / 2<R<R^{\prime}$ ) sinks faster than the $100 \%$ slip case and reach a steady-state value higher than any other test cases with $\beta<90^{\circ}$.
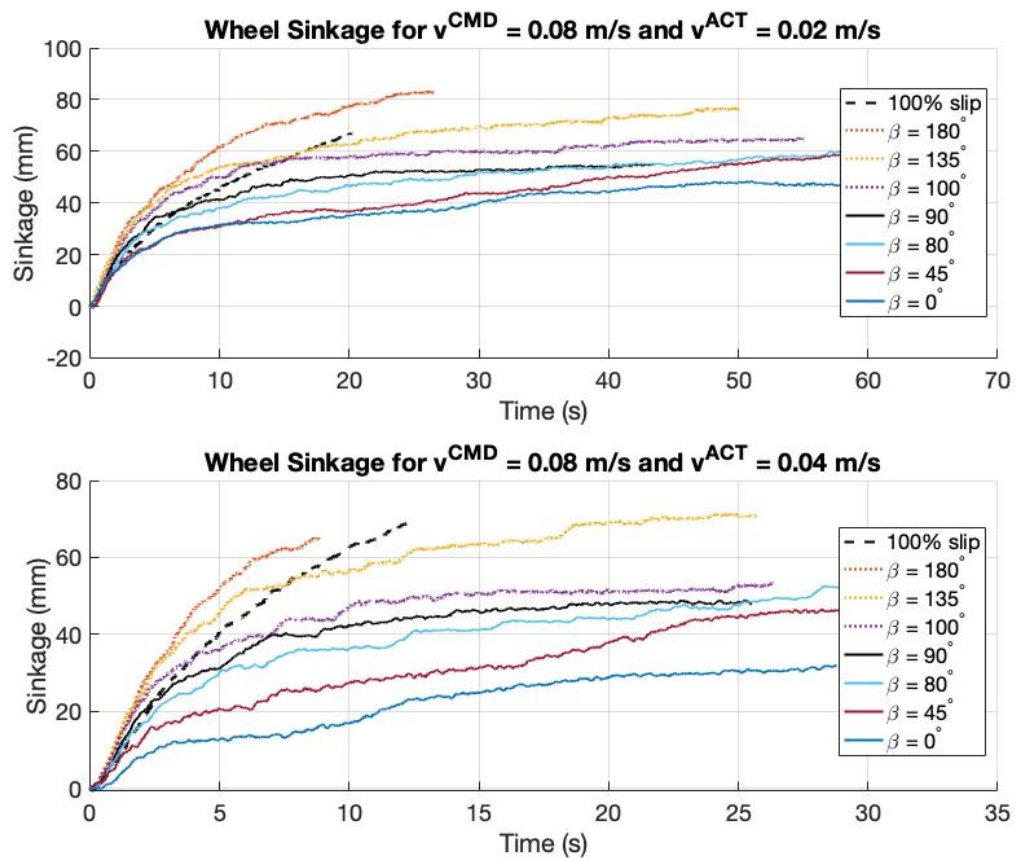

Figure B.1 Relative sinkage for a linear path with $v^{C M D}=0.08 \mathrm{~m} / \mathrm{s}$ and varying slip angle $\beta$ for $v^{A C T}=0.02 \mathrm{~m} / \mathrm{s}$ (top) and $v^{A C T}=0.04 \mathrm{~m} / \mathrm{s}$ (bottom). 
Further linear path tests were performed to validate that the inner wheels sink more in the special case, when $\beta>90^{\circ}$. Fig. B.2 depicts the relative sinkage for three set of slip angle. For each set, the actual velocity was kept at $0.04 \mathrm{~m} / \mathrm{s}$ and the rotation of the wheel was simply set to $v^{C M D}=0.08 \mathrm{~m} / \mathrm{s}$ for the $\beta<90^{\circ}$ angle and to $v^{C M D}=-0.08 \mathrm{~m} / \mathrm{s}$ for the $\beta>90^{\circ}$ angle.
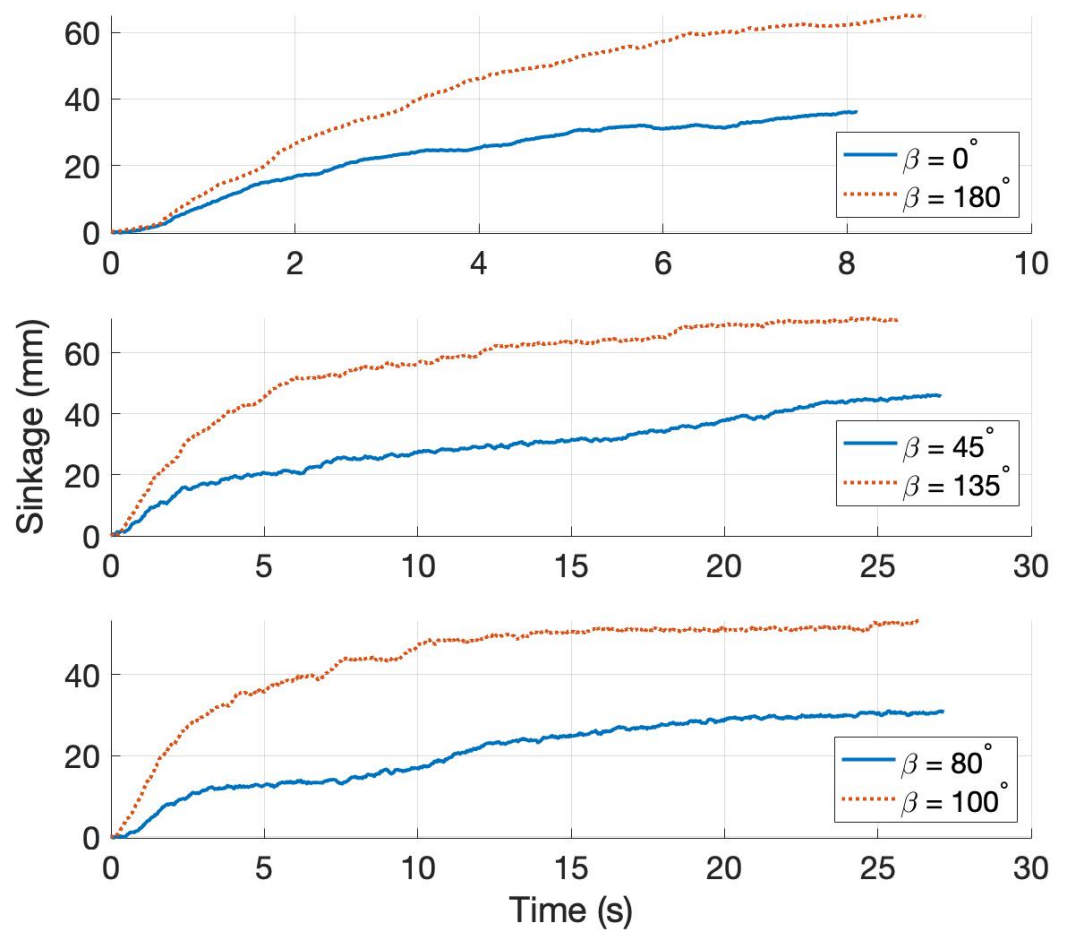

Figure B.2 Relative sinkage over time comparison when the wheel is rotating in opposite direction, for three set of slip angle $\beta$. 


\section{Appendix C}

\section{Other CLC vs. PLP Results}

Following up on the work presented in Section 6.1, additional CLC and PLP path were tested with the Clearpath Husky on hard concrete ground and on the sand of the CSA Mars analogue terrain. Moreover, as seen in Fig. C.2, new paths combining a circle, a straight line and a point turn (CLP and PLC) were performed to compare the total energy consumption.

Fig. C.1 and C.2 first show the energy difference, from the PLP to the CLC/CLP/PLC path as a function of $R_{A C T} / R_{A C T}^{\prime}$ for a $60^{\circ}-8 \mathrm{~m}-30^{\circ}$ path on hard ground and on sand, respectively. Then, the results from a $90^{\circ}-10 \mathrm{~m}-90^{\circ}$ path on sand are shown in Fig. C.3. Finally, the same path shown in the main body of the thesis, i.e. $45^{\circ}-10 \mathrm{~m}-45^{\circ}$, was tested using the $v_{m} a x$ constraint discussed in Section 2.1.1 to validate that similar results are obtained with a different velocity constraint that the constant total absolute velocity $v_{c}$ used throughout the thesis. 


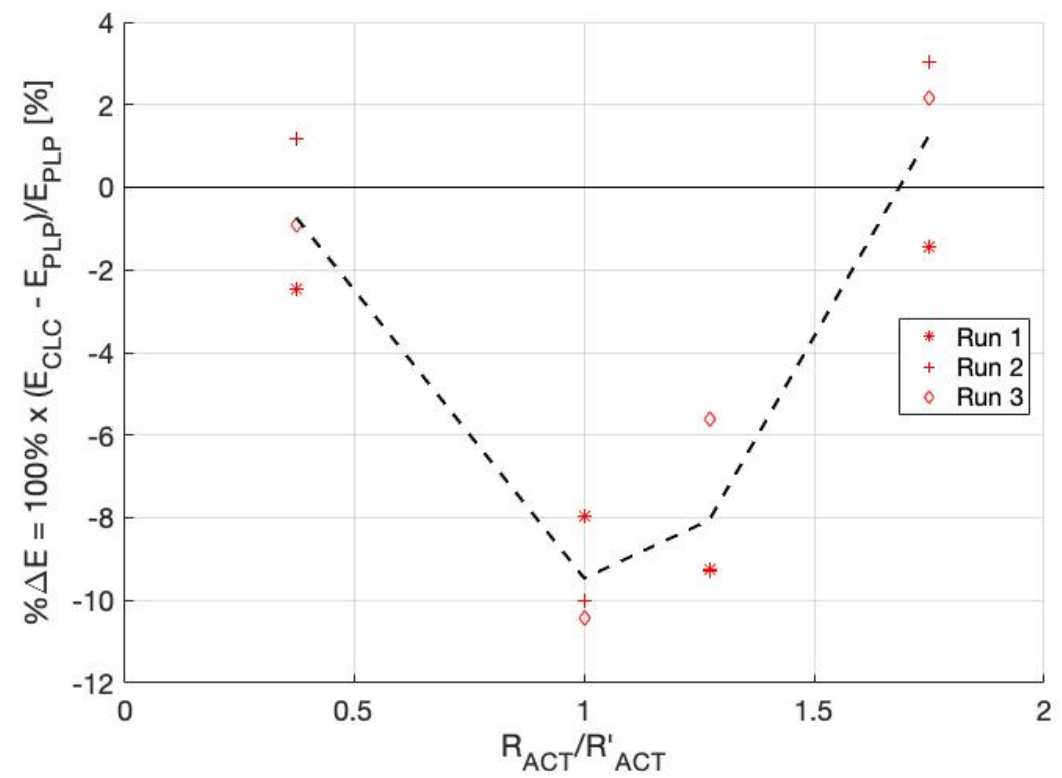

Figure C.1 Energy difference from the PLP to the CLC path with varying turning radius, depicted as $R_{A C T} / R_{A C T}^{\prime}$ for a $60^{\circ}-8 \mathrm{~m}-30^{\circ}$ path, on hard ground, with constant $v_{c}=0.3 \mathrm{~m} / \mathrm{s}$. The biggest energy reduction occurs at $R_{A C T}=R_{A C T}^{\prime}$.

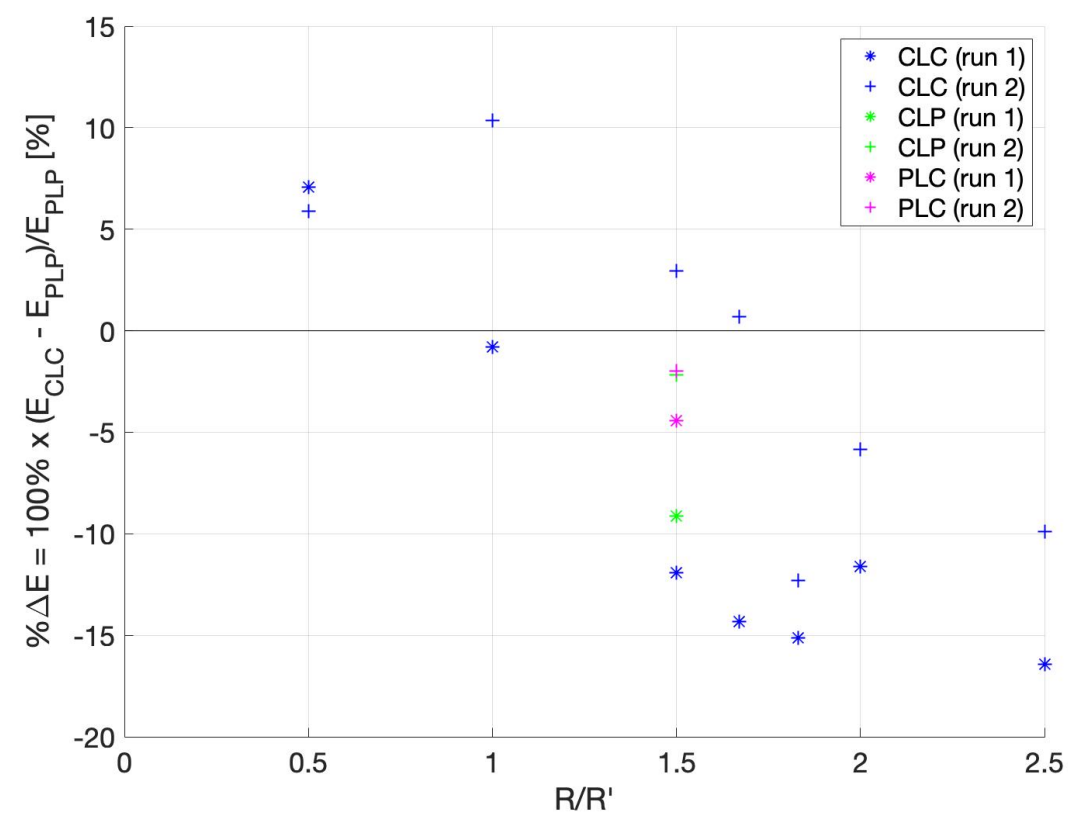

Figure C.2 Energy difference from the PLP to CLC/CLP/PLC path with varying turning radius, depicted as $R_{A C T} / R_{A C T}^{\prime}$ for a $60^{\circ}-8 \mathrm{~m}-30^{\circ}$ path, on sand, with constant $v_{c}=0.3 \mathrm{~m} / \mathrm{s}$. The biggest energy reduction occurs at around $2 R_{A C T}^{\prime}<R_{A C T}<2.5 R_{A C T}^{\prime}$. 


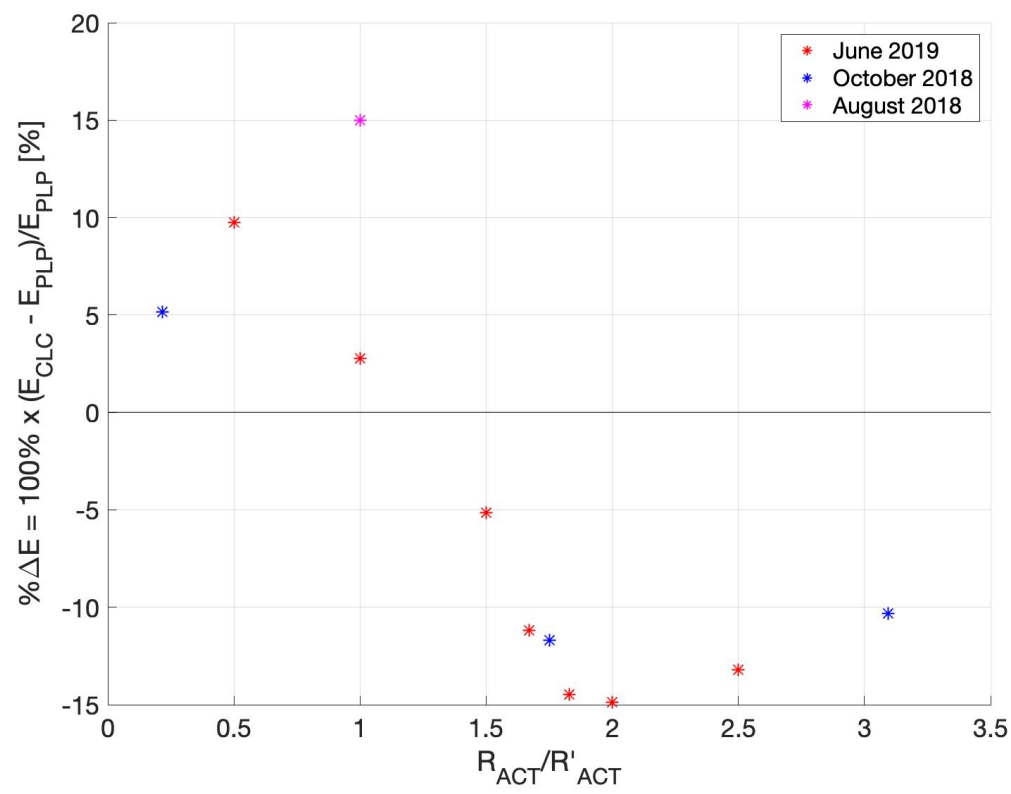

Figure C.3 Energy difference from the PLP to CLCpath with varying turning radius, depicted as $R_{A C T} / R_{A C T}^{\prime}$ for a $90^{\circ}-10 \mathrm{~m}-90^{\circ}$ path, on sand, with constant $v_{c}=0.3 \mathrm{~m} / \mathrm{s}$. The biggest energy reduction occurs at around $R_{A C T} \approx 2 R_{A C T}^{\prime}$.

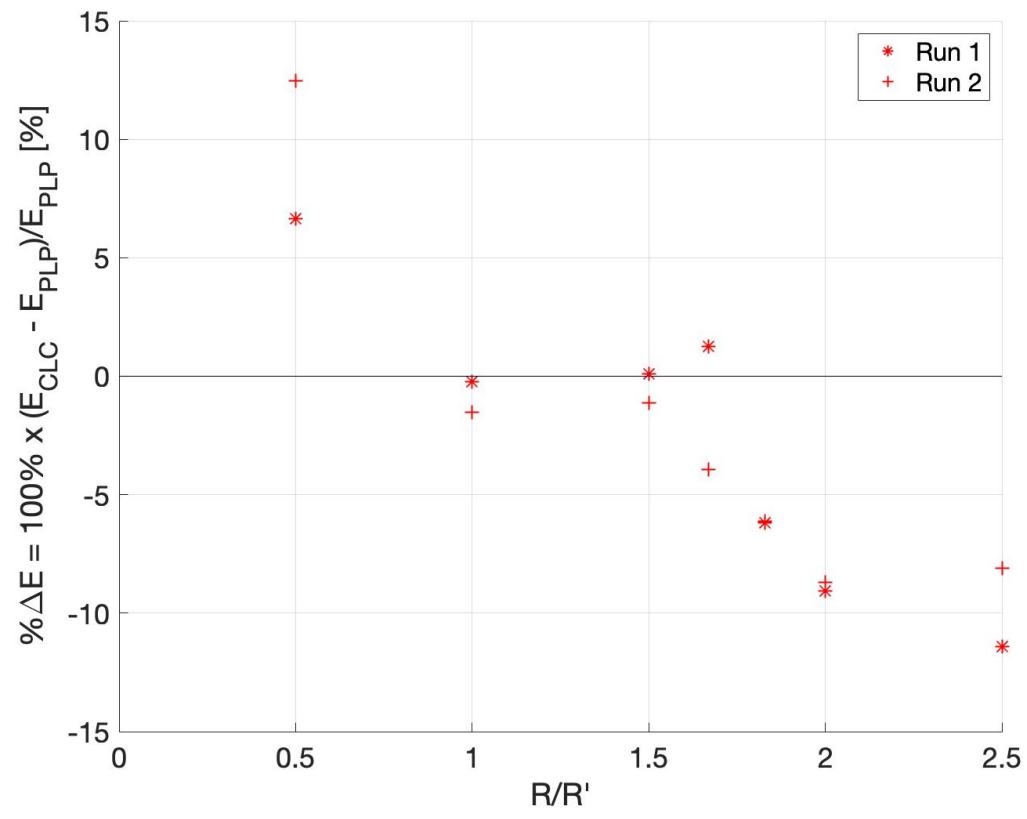

Figure C.4 Energy difference from the PLP to CLCpath with varying turning radius, depicted as $R_{A C T} / R_{A C T}^{\prime}$ for a $45^{\circ}-10 \mathrm{~m}-45^{\circ}$ path, on the sand of the CSA Mars analogue terrain, with constant $v_{\max }=0.3 \mathrm{~m} / \mathrm{s}$. The biggest energy reduction occurs at around $2 R_{A C T}^{\prime}<R_{A C T}<2.5 R_{A C T}^{\prime}$. 


\section{Appendix D}

\section{Evaluating the Slip Track}

In this appendix, the actual and commanded turning radii, i.e. $R_{A C T}$ and $R_{C M D}$, are first compared by plotting $R_{A C T}$ vs. $R_{C M D}$. These results are further used to have a quick estimation of the slip track $\left(B_{S}\right)$ for a particular terrain instead on using the Extended Kalmann Filter (EKF) to find the location of the left and right ICR (presented in Section 1.1.1).

Fig. D.1 shows the results for the two set of $90^{\circ}$ turns performed with the Clearpath Husky platform, on hard concrete ground.

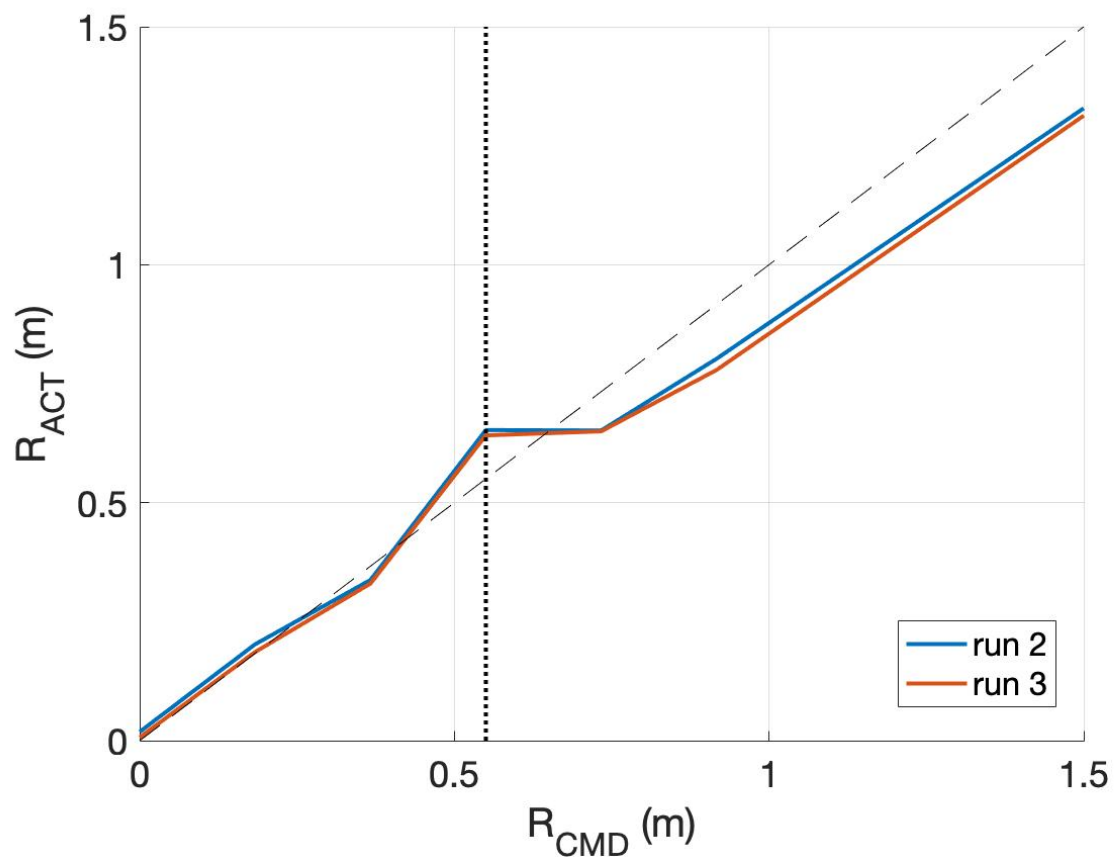

Figure D.1 Comparison between the commanded and actual turning radius, $R_{C M D}$ and $R_{A C T}$, for two runs of turns with the Clearpath Husky, on hard ground. 
Ideally, the measured $\left(R_{A C T}\right)$ vs. commanded $\left(R_{C M D}\right)$ turning radii curve should be along the diagonal (dotted line). However, for the Argo tests on sand shown in Fig. ?? (blue and orange lines), it clear that the measured turning radius is greater than the commanded turning radius. This could be caused by the fact that Argo controller doesn't consider slip and use the width $B$ of the rover to obtain the left and right wheels velocities, $V_{L}$ and $V_{R}$ respectively, from the commanded linear velocity $v_{x}$ and angular velocity $\omega_{z}$. The procedure to derive $V_{l}$ and $V_{r}$ is shown in Appendix A, using $B_{s}=B$ in the case of the Argo. Since $B$ is smaller than the actual sand slip track, the commanded turning radius will be smaller than the actual radius since $B_{S}$ increases as slip increases.

For the Husky, the actual turning radii is closer to the commanded value, for the tests performed in the sandbox. This is due to the fact that the controller use a slip track value of $B_{s}=1.1 \mathrm{~m}=2 B$ which is closer to the actual slip track of the Husky in the GRC-1 soil simulant. It should also be noticed that the measured radii from the CSA test campaign were smaller than the commanded turning radii. This could be explained by the fact that the sand from the CSA mars emulation terrain was wet and compacted, which reduces the slip encountered during the maneuvers. Accordingly, the actual slip track of the Husky in this terrain is less than the value used by the controller.

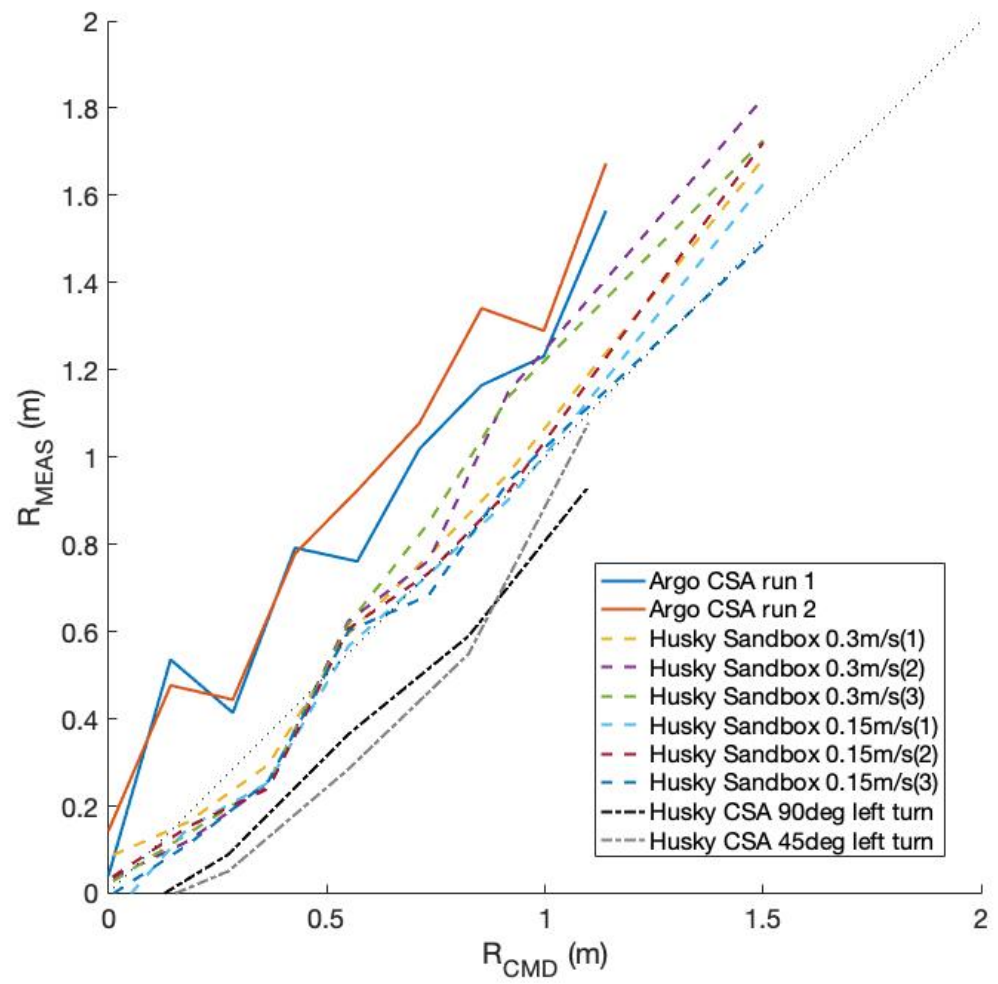

Figure D.2 $R_{A C T}$ vs. $R_{C M D}$ for all tests

As mentioned, the actual turning radius measured when $R_{C M D}=R^{\prime}$ was used to estimate the 
slip track $B_{s}$ of all the terrain on which a set of $90^{\circ}$ turns was performed. Table D. 1 presents the results of this analysis with the internal slip track of the Husky, used by the controller to convert inputted linear $v_{y}$ and angular $\omega_{z}$ velocities to left and right commanded velocities, i.e. $V_{l}$ and $V_{r}$ respectively (following equations of Appendix A). As the $R_{A C T}$ vs. $R_{C M D}$ curves were close to the diagonal dotted line in the two previous figures for the case of the laboratory sandbox and the hard ground, the resulting slip track for these two terrains is close to the internal slip track of $1.1 \mathrm{~m}$.

Table D.1 Estimated slip track using $R_{A C T}$ when $R_{C M D}=R^{\prime}$

\begin{tabular}{ll}
\hline Terrain & Slip Track $\left(B_{s}\right)$ \\
\hline Internal & $1.1 \mathrm{~m}$ \\
Laboratory sandbox & $1.21 \mathrm{~m}$ \\
CSA Mars analogue terrain & $0.65 \mathrm{~m}$ \\
Hard concrete ground & $1.29 \mathrm{~m}$ \\
\hline
\end{tabular}

The data gather in a CSA Mars analogue test campaign in 2017 was used to performed the ICR EKF presented in the work of [4]. The results of all the turns is shown in Fig. D.3 as a function of turning radius $R$, along with an average position of the left and right ICR along the vehicle's $X$ axis. The average slip track obtained is $1.78 \mathrm{~m}$, which is significantly higher than what was estimated in Table D.1.

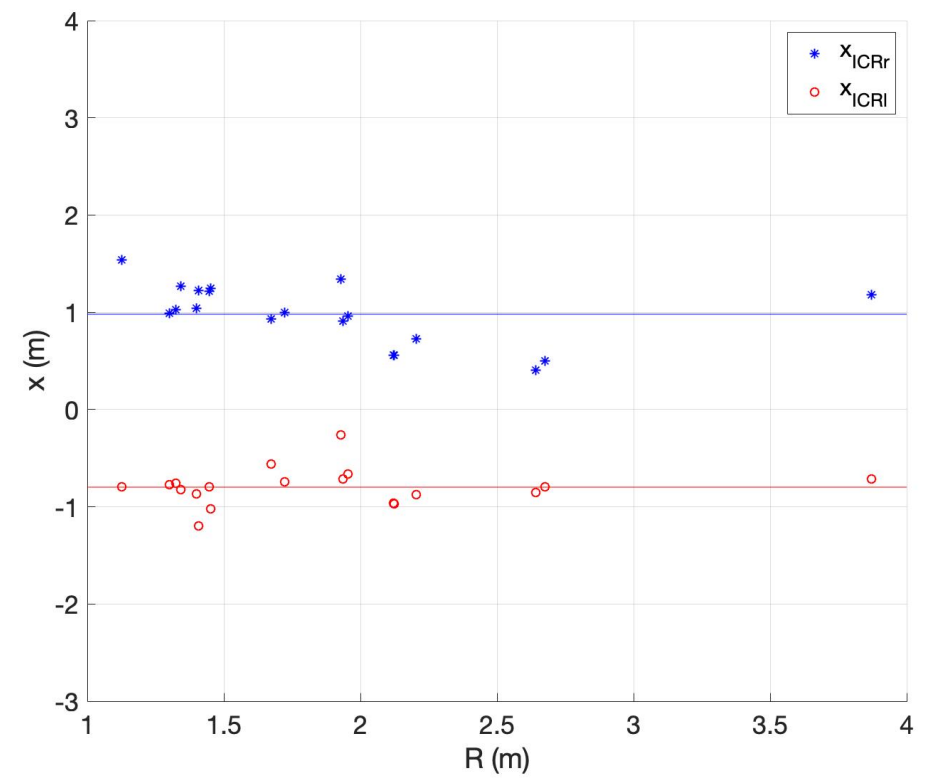

Figure D.3 ICR locations obtained for all test cases of the CSA 2017 test campaign 


\section{Appendix E}

\section{Unanswered Research Questions}

During this research, a few research questions were not answered. Two of them, which are interesting study areas, are presented in this appendix.

First, a phenomenon that wasn't explained in this work comes from the experimentally obtained slip track $B_{s}$ for the different terrains shown in Appendix C. From Table D.1, it can be noticed that the slip track measured on hard concrete ground is greater than the slip track for any other terrain tested, including the laboratory sandbox GRC-1 simulant. Does this means that a rotating wheel on loose soil slips more but skids less than on hard ground? Is this due to the fact that a sinking wheel can't perform lateral motion as easily as on a flat hard surface? Therefore, the overall skidding of a vehicle (or its slip track) would depend on the amount of sinkage experienced by its wheels, which vary depending on the maneuver. However, this contradicts the work of Martinez et al., which state that the ICRs remain bounded to a small area, regardless of the maneuver [1].

Furthermore, it wasn't explained why the measured angular velocity of the rover initially decreases but then increases again for all the turns except $2 R^{\prime} / 3$ (the turing radius in the special range identified), as seen in Fig. 6.9. What phenomenon can explain this? Does the increase occurs when the wheel sinkage reach its steady-state?

Finally, the wheel unit DC motor current measurements gathered during the set of circular arc path performed with the single-wheel test bed are shown in Fig. E.1. Since these currents are collected at the motor gate (instead of the controller gate with the Clearpath Husky), this current is proportional to torque. However, the clear increase in torque for the special case turn (reported by the torque-proportional curve of Fig. 5.7) is not seen here, as the current of the $2 R^{\prime} / 3$ case is similar to the $R^{\prime} / 3$ case. Is this because single-wheel test bed is using other motors to control the displacement of the wheel whereas for the Husky, the wheel DC motors are responsible for the wheel rotation and their displacement? Further investigation could be made in this area. 


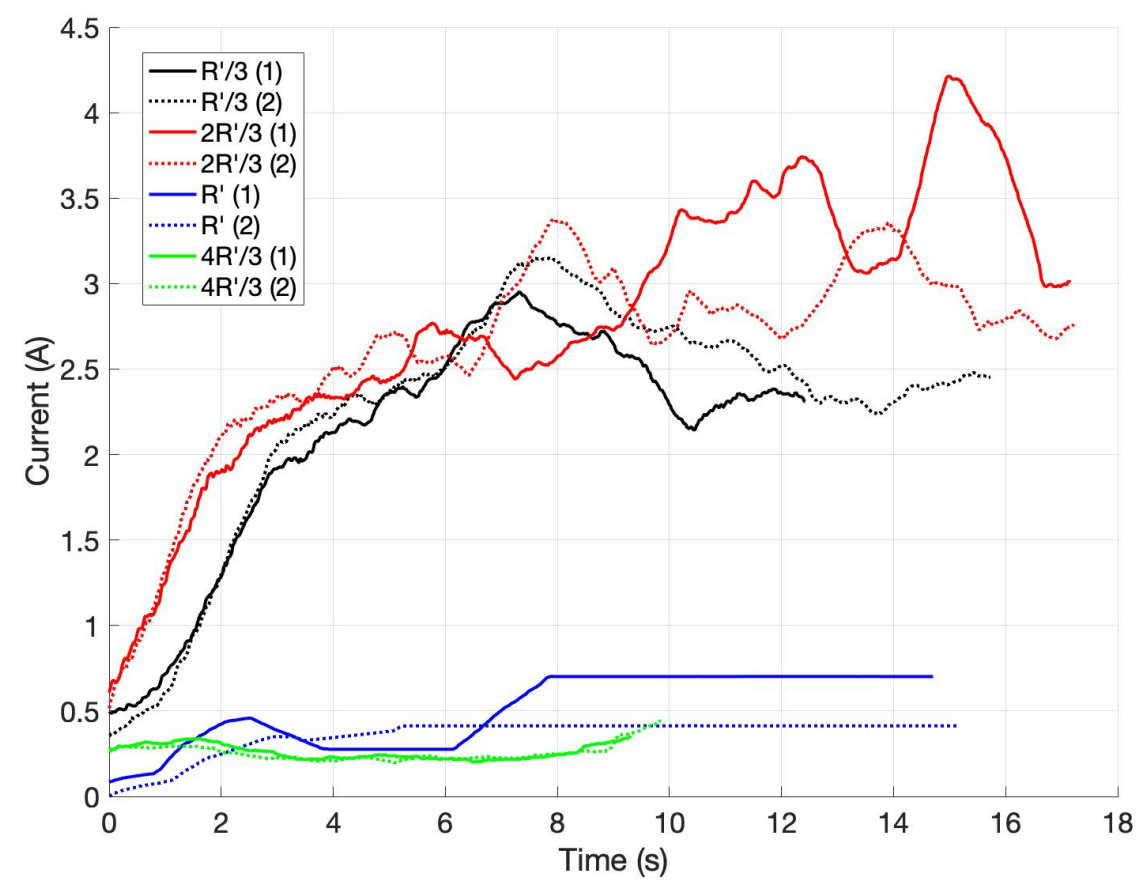

Figure E.1 Current consumption of the wheel motor of the five-axis single-wheel test bed. 
Appendix F

Submitted Journal Paper 
Noname manuscript No.

(will be inserted by the editor)

\title{
Considering Slip-Track for Energy-Efficient Paths of Skid-Steer Rovers
}

\author{
Meysam Effati • Jean-Sebastien Fiset • \\ Krzysztof Skonieczny
}

the date of receipt and acceptance should be inserted later

\begin{abstract}
Skid-steer rovers consume a lot more power in point turns compared to straight line motion. As energy is the integral of power over time, the turning radius should be considered explicitly for this type of rover. Lower instantaneous power consumption for wider arcs must be traded off against shorter traversal distance for tighter arcs by evaluating the total energy consumed when following different paths. This research seeks to find the most energy-efficient path from among Circular arc - Line - Circular arc (CLC) paths, a generalization of Point turn - Line - Point turn (PLP) paths which are the simplest path to execute for a skid-steer rover traversing between general start and end poses. The optimally energy-efficient CLC path on hard ground is found to have circular arcs of radius $R^{\prime}$, the turning radius at which a skid-steer rover's inner wheels are not commanded to turn. The radius corresponds to exactly half the rover's slip-track. Theoretical, numerical, and experimental evidence is presented to support this result. Further, important features of the $R^{\prime}$ turning radius are explored.
\end{abstract}

Keywords Energy-efficient paths · Skid-steer rovers · Optimization · Power modeling

\section{Introduction}

Skid-steering is a method of steering where four (or more) wheels are not themselves steered, but different velocities are commanded for the left and

The authors are with the Electrical and Computer Engineering Department, Concordia University, Montreal, QC, Canada.

Address: 1455 de Maisonneuve Blvd. W. Montreal, QC, H3G 1M8.

Tel.: +1 514-848-2424 ext. 3122

Email: m_effati@encs.concordia.ca;j_fise@encs.concordia.ca;kskoniec@encs.concordia.ca

Address(es) of author(s) should be given 
right wheels in order to maneuver a vehicle. Due to their mechanical simplicity, maneuverability and robustness, skid-steer rovers are widely used for excavation and loading, planetary exploration [1][2] and other field robotics applications. Energy-efficient navigation is an important aspect of any of these applications, especially when autonomously planning paths in power-starved environments. However, the power consumption for skid-steer rovers can be high, and also highly variable, compared to other steering mechanisms (such as explicit or Ackerman steering) due to the torque required to overcome lateral motion resistance while skidding in a turn.

A key challenge of skid-steer mobility is thus the power and energy consumption of this steering configuration. Shamah et al. [3] show that during a maneuver the power consumption of a skid-steer rover is generally inversely related to its turning radius, with lowest power consumption during straight line driving and highest during tight turns. For point turns (i.e. turning in place), skid steering can require over twice the power of explicit steering [3]. The difference between the two steering modes drops away with increasing turning radius. Thus, the shortest distance path, which is a Point turn - Line - Point turn (PLP) path, may not be the lowest-energy path. In fact, we show this to be the case.

Studying energy efficiency of skid-steer rovers with respect to their turning radii provides insights that are useful for path planning.

1.1 Sample-based and local planning methods

In general path planning for vehicles, differential constraints and complex environments motivate sampling-based approaches (e.g. Rapidly-exploring Random Trees (RRT)). However, there is also a need to generate feasible local paths between the nodes that sampling-based algorithms generate. Accordingly, Local Planning Methods (LPM) provide useful motion primitives for the global planning method [4]. These local paths are generated in an obstacle-free environment, representing the space between nodes that are already chosen to avoid obstacles in a complex environment. The simplest LPM for skid-steer rovers to transition between general local start and end poses is a PLP path, which can be generalized to Circular-arc - Line - Circular-arc (CLC) paths. It is possible to explicitly optimize these simpler local paths (in terms of either length, time duration, energy consumption, etc.). LaValle describes two probable advantages of considering such optimal local paths to build overall paths [4]:

1. They can lead to more time/length/energy efficient overall paths

2. They can be more efficient in computational cost compared to executing the global algorithms with ad hoc local paths

There are many different numerical sampling-based methods to search for efficient paths in complex environments (such as in the presence of obstacles), including potential fields [5], several versions of rapidly exploring random tree 
algorithms (such as RRT [6][7][8], RRT* [9], and Theta*-RRT [10]), and several numerical methods based on heuristic approaches (such as $\mathrm{D}^{*}[11]$ ). Sampling Based Model Predictive Optimization (SBMPO) [12] is popular for path planning with skid-steer rovers. Because SBMPO samples from the feasible space of control inputs, it can automatically satisfy any kinematic or dynamic constraints. SBMPO generates nodes on a graph where a model predicts the rover would be after the sampled control inputs have been applied for some specified duration. The cost at each node is the sum of the cost of getting to that node (which can be predicted by the model used) plus an "optimistic" estimate (i.e. meant to guarantee to not over-estimate) of the remaining cost to get to the goal from that node. After a node is expanded with some fixed number of new nodes and each is assigned a cost, a priority queue is re-sorted to pick the next node to expand (the one with lowest cost). Reese [13] proved that SBMPO gives the optimal path on the graph, but only once the priority queue is completely exhausted.

Recent attempts to determine energy-efficient paths for skid-steer rovers use SBMPO. Gupta et al. [14] opt to select the first path that reaches the goal by this method (i.e. leaving nodes still available in the priority queue), resulting in a suboptimal trajectory, in order to reduce computation time. Pentzer et al. [15] assume that a point turn followed by a straight line (a subset of the PLP path type) to the goal (which, in general, may still be far away) provides an "optimistic" estimate of remaining energy cost; however, our present work, using the same power model, will show that there exist paths with lower cost than this option and thus it does not in fact guarantee not to over-estimate cost. Further, even a fully optimally implemented SBMPO provides only the optimal solution on the graph of sampled nodes. To approach a global optimum in continuous space, the sampling must thus be dense. However, it is mentioned by Gupta et al. [14] that computation time is already a limiting consideration. These challenges in practice related to suboptimality and computational cost align with the points raised by LaValle [4] motivating optimal local paths. The next subsection introduces CLC paths for this purpose.

In other related work, Tokekar et al. [16] work toward optimizing energy consumption for car-like robots (i.e. with Ackerman steering); they first show how to analytically find energy-optimal velocity profiles along a given path, and then build a discretized graph composed of individual circular arcs (or straight lines) connecting the vertices. Shortest path LPMs have been developed for car-like rovers [17,18] as well as differential-drive rovers [19]; shortest time LPMs have also been developed for differential-drive rovers [20]. In all these cases, the optimal solutions consist of sequences of straight lines, circular arcs and/or point turns.

\subsection{Path types and special turning radii}

In order to study the impact of turning radius on skid-steer rover energy efficiency, a pre-defined class of paths inspired by the results of the work described 
above, composed of CLC segments, is considered. CLC paths are a generalization of PLP paths, where the initial and final point turns can be replaced with arcs of any turning radius, $0<R<\infty$. The reasons for considering this particular type of pre-defined path include:

- Turning radius has an important effect on skid-steer rover power consumption [3]. A practical approach to controlling turning radius is to have constant-turning-radius paths by using circular arcs.

- PLP paths are the simplest paths a skid-steer rover can employ for motion from a general start to end pose, and are thus very commonly used in practice. The generalization of this concept beyond $R=0$ explores possible optimizations while retaining this option as a possible solution.

- In prior related problems, CLC or PLP paths are optimal (in terms of shortest distance [17] or shortest time [20]) as long as start and end poses are sufficiently separated. But, they are not investigated in terms of energyefficiency.

Earlier work by the authors [21] considered predefined classes consisting only of 2 circular arcs, but this turned out to rarely be optimal, especially for paths of any appreciable length where including a straight line can significantly reduce the overall path length.

In skid-steer rovers, different turning radii are achieved by commanding different wheel velocities to the left and right sides of the rover. $V_{l}$ and $V_{r}$ are the left and right wheel velocities (i.e. wheel angular velocities multiplied by wheel radius), respectively, and are control inputs. The boundary between the cases where $V_{l}$ and $V_{r}$ are of the same or of opposite sign (i.e. when either $V_{l}$ or $V_{r}$ are 0 ) turns out to be very important. The turning radius at this boundary is defined as $R^{\prime}$ in this work (and is equal to half the rover's slip track, as will be shown in the following section). The concept is somewhat similar to $R_{c}$ recently introduced by Dogru and Marques [22], but $R^{\prime}$ is more general and its importance is more fully analyzed here than any related concepts in prior literature. $R_{c}$ is assumed to occur at half the rover width, not accounting for the possibility of lateral slip. Further, Dogru and Marques note that when $R_{c}<R<\infty$, the side with the faster wheels drags the other side along, resulting in negative power consumption on one side of the robot. As will be shown in Section 4.4 of this work, the range of turning radii for which this is true has tighter bounds in general. Also, although Dogru and Marques work toward finding an optimal turning radius for skid-steer rovers in subsequent work [23], they do not explicitly link the important boundary case (i.e. $R_{c}$ or, actually, $\left.R^{\prime}\right)$ to optimal energy consumption. A contribution of this work is thus that it provides deeper context and explanations for those earlier results in the literature.

\subsection{Contributions and organization}

A key contribution of this work is a detailed exposition of the importance of $R^{\prime}$, including its use in optimally energy-efficient local path planning on hard 
ground. When considered with respect to a popular power model for skid-steer rovers [24], $R^{\prime}$ corresponds to an important transition for energy consumption: below $R^{\prime}$ energy consumption is not a function of turning radius while above $R^{\prime}$ it is. CLC paths, with the ' $\mathrm{C}$ ' segments consisting of circular arcs of radius $R^{\prime}$, are found to be optimally energy efficient in both simulation and experiments on hard ground.

In addition, it is explicitly shown that there are CLC paths that are more energy-efficient than point turn-line-point turn (PLP) paths on hard ground. Thus $R^{\prime}$-CLC paths (CLC paths with $R=R^{\prime}$ ) should be used as "optimistic" estimates of cost to a goal, rather than PLP paths.

In section 2, the problem of theoretical and experimental investigation for skid-steer rovers' energy-efficient CLC paths are defined. The kinematics and power model of the problem are also introduced. Then, section 3 is specified to the mathematical formulation of energy-efficient CLC paths' problem. Afterwards, section 4 reports the numerical analysis and experimental tests for comparison of energy-efficient CLC with PLP paths. Finally, section 5 reports conclusions regarding the optimality of $R^{\prime}$ and the conditions in which energyefficient CLC paths consume less energy than PLP, and discusses future work.

\section{Problem Statement and System Definition}

To investigate the significance of $R^{\prime}$, the usefulness of CLC paths, and the relationship between these two contributions, the following problem is studied in this work.

Problem 1: Finding energy-efficient CLC (Fig. 1) paths for skid-steer rovers both theoretically (by using the existing power model) and experimentally (by commanding different CLC paths with Husky UGV). The subproblems can be summarized as follows:

i Computing the optimally energy-efficient CLC path by theoretical analysis, and comparing these results to PLP (Fig. 2) paths (shortest path).

ii Experimentally measuring the energy required to perform PLP paths and CLC paths with turning radii at and around those suggested by the analysis in Problem 1-i, on hard ground.

iii Comparing the results of Problem 1-i and Problem 1-ii.

Problem 1-i is further explicitly defined here:

$$
\begin{array}{ll}
\underset{R_{1}, R_{2}, \theta_{B_{1}}}{\operatorname{minimize}} & E \\
\text { subject to } & h_{i}=0, i=1 . . n \\
& g_{j} \leq 0, j=1 . . m
\end{array}
$$

where $E, h_{i}$, and $g_{j}$ are the energy consumed during the path, and the equality constraints and inequality constraints (defined in subsection 3.2), respectively. Also, $n$ and $m$ are the numbers of equality and inequality constraints, respectively. In addition, $R_{k}(k=1,2)$ and $\theta_{B_{1}}$ are the turning radii of the circles 


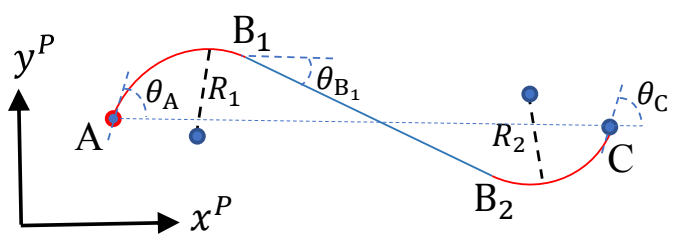

Fig. 1: A CLC path including two circular arcs and a line segment. The axes $x^{P}$ and $y^{P}$ indicate the path-defined coordinate system. Also, $\mathrm{B}_{1}$ and $\mathrm{B}_{2}$ are the starting and end point of the straight line segment.

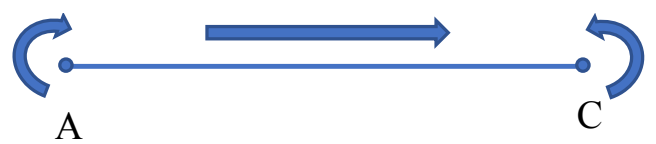

Fig. 2: A PLP path consisting of two point turns and a line segment

and angle of the straight line (with respect to the path-defined coordinate system) in Fig. 1, respectively.

\section{Assumptions:}

- The rover is skid-steer (i.e. can turn with any turning radius, including point turns)

- The rover only moves forward on flat ground

- There are no obstacles (i.e. LPM)

- The path is $\mathrm{C}^{2}$ differentiable

$-\mathbf{x}(0)=\mathbf{x}_{A}$ and $\mathbf{x}\left(t_{f}\right)=\mathbf{x}_{C}$ for $\mathbf{x}=[x, y, \theta]^{T}$, where $(x, y)$ and $\theta$ are the position and heading angle of the rover in the global coordinate system, respectively. Also, $t_{f}$ is the final time. Moreover, $\mathbf{x}_{A}$ and $\mathbf{x}_{C}$ are known.

Further assumptions are employed purely to simplify the analysis of the example cases studied:

- The rover center of mass is located at its centroid

- Both $\theta_{A}$ and $\theta_{C}$ are in the same quadrant (in the path-defined coordinate system, whose $\mathrm{x}$-axis is aligned with the line connecting points $\mathrm{A}$ and $\mathrm{C}$, see Fig. 1)

\subsection{Skid-steer rover kinematics}

Rover kinematics can be defined based on the concept of Instantaneous Centers of Rotation (ICR) [25]. The parameters $\left(x_{I C R v}, y_{I C R v}\right),\left(x_{I C R r}, y_{I C R r}\right)$, 


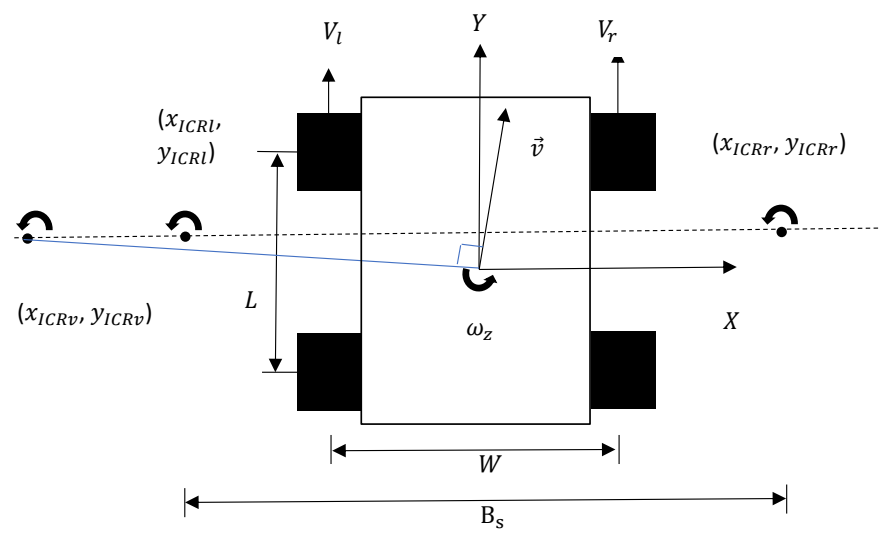

Fig. 3: A schematic figure of a skid-steer rover and its associated instantaneous centers of rotation (ICRs).

and $\left(x_{I C R l}, y_{I C R l}\right)$ are the vehicle, right hand-side, and left hand-side ICR positions, respectively. These parameters are shown in Fig. 3 and are as follows:

$$
\begin{gathered}
x_{I C R v}=-\frac{v_{y}}{\omega_{z}} \\
x_{I C R l}=\frac{V_{l}-v_{y}}{\omega_{z}} \\
x_{I C R r}=\frac{V_{r}-v_{y}}{\omega_{z}} \\
y_{I C R v}=y_{I C R l}=y_{I C R r}=\frac{v_{x}}{\omega_{z}} \\
v_{y}=\frac{V_{r} x_{I C R l}-V_{l} x_{I C R r}}{x_{I C R l}-x_{I C R r}} \\
v_{x}=\frac{\left(V_{l}-V_{r}\right) y_{I C R v}}{x_{I C R l}-x_{I C R r}} \\
\omega_{z}=\frac{V_{l}-V_{r}}{x_{I C R l}-x_{I C R r}}
\end{gathered}
$$

Moreover, $v_{x}, v_{y}$, and $\omega_{z}$ are the velocity in $X$, velocity in $Y$, and angular velocity about $Z$ axes of the rover's body frame, respectively. Recall that $V_{r}$ and $V_{l}$ are the right and left wheel velocities (i.e. wheel angular velocities multiplied by wheel radius), respectively, and are control inputs. Martinez et al. [26] show that the positions of ICRs can be assumed to be approximately constant for a particular terrain type. They can be estimated by taking experimental measurements (as is done in this work, see subsection 4.2) or via dynamics simulations, for a particular soil type and narrow range of speeds. 
Furthermore, $W$ and $L$ are the distance between the center of left and right wheels in the $X$ direction and the distance between the center of front and rear wheels in the $Y$ direction, respectively. Also, the slip track $\left(B_{s}\right)$ is defined as follows:

$$
B_{s}=x_{I C R r}-x_{I C R l}
$$

and can also be seen in Fig. 3 .

Based on the assumptions listed before, the rover center of mass is located at its centroid. Accordingly, the following equations can be concluded:

$$
\begin{gathered}
x_{I C R r}=-x_{I C R l} \\
y_{I C R v}=y_{I C R l}=y_{I C R r}=0
\end{gathered}
$$

Hence, from (6) through (11) it can be concluded that

$$
\begin{gathered}
v_{x}=0 \\
v=v_{y}=\frac{V_{r}+V_{l}}{2} \\
\omega_{z}=\frac{V_{r}-V_{l}}{B_{s}} \\
x_{I C R r}=\frac{B_{s}}{2} \\
x_{I C R l}=-\frac{B_{s}}{2}
\end{gathered}
$$

From the fact that $|v|=R\left|\omega_{z}\right|$ as well as equations (13) and (14), the turning radius can be expressed as:

$$
R=\frac{B_{s}}{2}\left|\frac{V_{r}+V_{l}}{V_{r}-V_{l}}\right|, V_{r} \neq V_{l}
$$

Definition $1 R^{\prime}=\frac{B_{s}}{2}$. Hence, $R=R^{\prime}$ is the boundary between the separate cases where $V_{r}$ and $V_{l}$ are either of equal or opposite sign.

As mentioned before, $R^{\prime}$ is the turning radius at which a skid-steer rover's inner wheels are not commanded to turn. Without loss of generality consider the right wheel as the inner wheel. Therefore, at $R^{\prime}$ the right velocity should be zero $\left(V_{r}=0\right)$. Hence, using Eq. (17), $R^{\prime}=\frac{B_{s}}{2}\left|\frac{V_{l}}{-V_{l}}\right|=\frac{B_{s}}{2}$. 


\subsection{Power Model}

This subsection adapts a popular existing skid-steer power model for usage in Problem 1-i. This model is based on a frictional wheel-terrain contact assumption. This power model, developed by Morales et al. ([24][27]) and used by Pentzer et al. ([15][28]), is as follows:

$$
P=\mu\left|\omega_{z}\right| \sum_{n=1}^{\mathrm{N}}\left(p_{n}|| \mathbf{a}_{\mathbf{n}}-\mathbf{C}_{r, l}||\right)+G\left(\left|V_{r}\right|+\left|V_{l}\right|\right)
$$

where $P$ is power consumed, $\mu$ is the friction coefficient, $\omega_{z}$ is the angular velocity, $p_{n}$ is the normal force on each wheel, $V_{r}$ and $V_{l}$ are right and left wheel velocities, $\left\|\mathbf{a}_{n}-\mathbf{C}_{r, l}\right\|$ (Fig. 4) is the distance from the right or left Instantaneous Center of Rotation (ICR) to a wheel, $G$ is the internal and rolling resistance coefficient, and $\mathrm{N}$ is the number of wheels. $V_{r}$ and $V_{l}$ are the inputs for the power model. This power model, using Fig. 4, can be expanded as follows:

$$
\begin{array}{r}
P=\mu\left|\omega_{z}\right| p\left(\sqrt{\left(\frac{L}{2}+y_{I C R r}\right)^{2}+\left(\frac{W}{2}-x_{I C R r}\right)^{2}}\right. \\
+\sqrt{\left(\frac{L}{2}-y_{I C R r}\right)^{2}+\left(\frac{W}{2}-x_{I C R r}\right)^{2}} \\
+\sqrt{\left(\frac{L}{2}+y_{I C R l}\right)^{2}+\left(\frac{W}{2}+x_{I C R l}\right)^{2}} \\
+\sqrt{\left.\left(\frac{L}{2}-y_{I C R l}\right)^{2}+\left(\frac{W}{2}+x_{I C R l}\right)^{2}\right)} \\
+G\left(\left|V_{r}\right|+\left|V_{l}\right|\right)
\end{array}
$$

where $p$ is the equal normal force applied to each wheel. The other parameters are as defined before.

Using (11), (15) and (16), the power model can be simplified as follows:

$$
P=2 \mu\left|\omega_{z}\right| p \sqrt{L^{2}+\left(W-B_{s}\right)^{2}}+G\left(\left|V_{r}\right|+\left|V_{l}\right|\right)
$$

or,

where $F$ is

$$
P=F\left|\omega_{z}\right|+G\left(\left|V_{r}\right|+\left|V_{l}\right|\right)
$$

$$
F=2 \mu p \sqrt{L^{2}+\left(W-B_{s}\right)^{2}}
$$

Note that the absolute value functions present in Eq. (21), in conjunction with the definition of $\omega_{z}$ in Eq. (14), motivate looking separately at the cases were $V_{r}$ and $V_{l}$ are either of equal or opposite sign. From inspecting Eq. (17) in conjunction with Definition 1, the boundary between these separate cases (i.e. when either $V_{r}=0$ or $V_{l}=0$ ) corresponds to $R=R^{\prime}$. Theorem 1 in the next section thus splits the consideration of turning radii between those below or above $R^{\prime}$. 


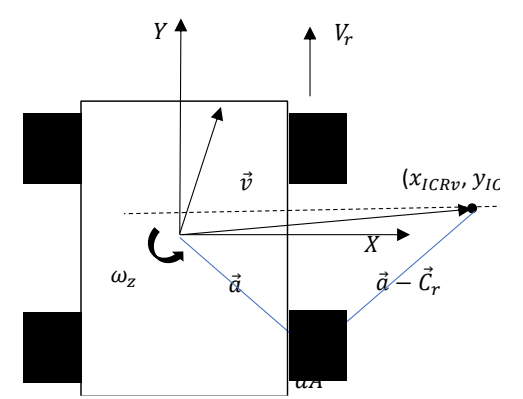

Fig. 4: Distance of a wheel from ICR. To calculate $\left\|\mathbf{a}_{n}-\mathbf{C}_{r, l}\right\|$ for each wheel, the ICR on the same side as the wheel is always considered (i.e. for the wheels in the right hand side of the rover, $\mathbf{C}_{r}$ should be considered.)

\section{Energy-Efficient CLC Paths}

This section focuses on the mathematical formulation of Problem 1-i (see section 2). Accordingly, the energy consumptions of a skid-steer rover during a circular-arc path segment and a straight line motion are formulated. Then, using these equations, the optimization problem for an energy-efficient CLC path with all of its equality and inequality constraints is defined.

\subsection{Energy Consumption During a Circular-arc and a Straight Line}

It is known that energy $(E)$ can be obtained by the following equation.

$$
E=\int P d t
$$

where $t$ is time. In addition, along a circular arc and a straight line, the related time is respectively equal to:

$$
\begin{aligned}
& t=\int\left|\frac{d \theta}{\omega_{z}}\right| \\
& t=\int\left|\frac{d s}{v}\right|
\end{aligned}
$$

where $d \theta$ and $d s$ are the differential of angle and displacement of the rover, respectively.

Theorem 1 The energy for the skid-steer rover when doing a circular arc maneuver (using the power model of Eq. (21)) is equal to:

$$
E_{\mathrm{C}}= \begin{cases}\left(F+G B_{s}\right)|\Delta \theta|, & \text { if } 0 \leq R \leq R^{\prime} \\ (F+2 R G)|\Delta \theta|, & \text { if } R>R^{\prime}\end{cases}
$$


Proof: Starting with the power model, (21), and the general equations (23) and (24), gives:

$$
\begin{aligned}
E_{\mathrm{C}} & =\int\left(F\left|\omega_{z}\right|+G\left(\left|V_{r}\right|+\left|V_{l}\right|\right)\right)\left|\frac{d \theta}{\omega_{z}}\right| \\
& =\int\left(F+G \frac{\left|V_{r}\right|+\left|V_{l}\right|}{\left|\omega_{z}\right|}\right)|d \theta|
\end{aligned}
$$

Using (14), this becomes:

$$
E_{\mathrm{C}}=\int\left(F+G B_{s} \frac{\left|V_{r}\right|+\left|V_{l}\right|}{\left|V_{r}-V_{l}\right|}\right)|d \theta|
$$

The definition of $R^{\prime}$ ensures that in the region $0 \leq R<R^{\prime}, V_{r}$ and $V_{l}$ are of opposite sign. Therefore, the following relation is always true:

$$
\frac{\left|V_{r}\right|+\left|V_{l}\right|}{\left|V_{r}-V_{l}\right|}=1
$$

Without loss of generality, consider $V_{r}$ positive. Then, $\left|V_{r}\right|=V_{r}$ and $\left|V_{l}\right|=$ $-V_{l}$. Hence, Eq. 29 can be proved. As a result, the energy is:

$$
\begin{aligned}
E_{\mathrm{C}} & =\int\left(F+G B_{s}\right)|d \theta| \\
& =\left(F+G B_{s}\right) \int|d \theta| \\
& =\left(F+G B_{s}\right)|\Delta \theta|, \text { for } 0 \leq R \leq R^{\prime}
\end{aligned}
$$

Therefore the energy for any turn with $0 \leq R<R^{\prime}$ is a constant times $|\Delta \theta|$. When $R>R^{\prime}$, it is known that both $V_{r}$ and $V_{l}$ have the same sign (both are positive or negative). Accordingly, it can be proved that equation (17) can be written in the following format:

$$
R=\frac{B_{s}}{2} \frac{\left|V_{r}\right|+\left|V_{l}\right|}{\left|V_{r}-V_{l}\right|}
$$

Hence, using Eq. (28), the energy for a constant radius arc is as follows:

$$
\begin{aligned}
E_{\mathrm{C}} & =\int(F+2 R G)|d \theta| \\
& =(F+2 R G) \int|d \theta| \\
& =(F+2 R G)|\Delta \theta|, \text { for } \quad R>R^{\prime}
\end{aligned}
$$


Accordingly, using Theorem 1, the energy function for arc of a circle with turning radius of $R$ can be written as follows:

$$
\begin{aligned}
E_{\mathrm{C}}= & \left(F+G B_{s}\right)|\Delta \theta|\left(H[R]-H\left[R-R^{\prime}\right]\right) \\
& +(F+2 G R)|\Delta \theta| H\left[R-R^{\prime}\right]
\end{aligned}
$$

where the Heaviside function for real numbers, $n$, is as follows:

$$
H[n]= \begin{cases}0, & \text { if } n<0 \\ 1, & \text { if } n \geq 0\end{cases}
$$

After more simplifications, the energy equation presented by Eq. (33) becomes as follows:

$$
E_{\mathrm{C}}=a_{1}^{\prime}|\Delta \theta| H[R]+\left(-a_{2}^{\prime}+a_{3}^{\prime} R\right)|\Delta \theta| H\left[R-R^{\prime}\right]
$$

where

$$
\begin{gathered}
a_{1}^{\prime}=F+\mathrm{G} B_{s} \\
a_{2}^{\prime}=G B_{s} \\
a_{3}^{\prime}=2 G
\end{gathered}
$$

Lemma 1 The energy for the skid-steer rover when doing a straight line maneuver (using the power model of Eq. (21)) is equal to:

$$
E_{\mathrm{L}}=2 G \sqrt{\left(x_{B_{1}}^{P}-x_{B_{2}}^{P}\right)^{2}+\left(y_{B_{1}}^{P}-y_{B_{2}}^{P}\right)^{2}}
$$

where $\left(x_{B_{1}}, y_{B_{1}}\right)$ and $\left(x_{B_{2}}, y_{B_{2}}\right)$ are the start and end of straight line maneuver, respectively.

Proof: It is known that $\omega_{z}=0$ for a straight line motion. Hence, starting with the power model, (21), and the general equations (23) and (25), gives:

$$
\begin{aligned}
E_{\mathrm{L}} & =\int\left(G\left(\left|V_{r}\right|+\left|V_{l}\right|\right)\right)\left|\frac{d s}{v}\right| \\
& =\int\left(G \frac{\left|V_{r}\right|+\left|V_{l}\right|}{|v|}\right)|d s|
\end{aligned}
$$

Using Eq. (13), this becomes:

$$
E_{\mathrm{L}}=\int\left(2 G \frac{\left|V_{r}\right|+\left|V_{l}\right|}{\left|V_{r}+V_{l}\right|}\right)|d s|
$$

Since $V_{r}$ and $V_{l}$ have the same sign at the straight line motion, the following relation is always true:

$$
\frac{\left|V_{r}\right|+\left|V_{l}\right|}{\left|V_{r}+V_{l}\right|}=1
$$

Therefore,

$$
\begin{aligned}
E_{\mathrm{L}} & =2 G \int|d s| \\
& =2 G|\Delta s| \\
& =2 G \sqrt{\left(x_{B_{1}}^{P}-x_{B_{2}}^{P}\right)^{2}+\left(y_{B_{1}}^{P}-y_{B_{2}}^{P}\right)^{2}}
\end{aligned}
$$




\subsection{Mathematical Formulations of Optimal CLC Paths}

This subsection sets up the optimization problem for finding enegry-efficient CLC paths (Fig. 5). The notation is based on prior work optimizing CircleCircle (CC) paths [21]; as points $\mathrm{A}$ and $\mathrm{C}$ grow farther apart, the motivation for this present work adding a straight line segment becomes clear.

By using Eq. (35), Lemma 1, and the assumptions described in section 2 the energy for the full path is obtained as follows:

$$
\begin{gathered}
E=E_{A B_{1}}+E_{B_{1} B_{2}}+E_{B_{2} C} \\
E=\left(a_{1}^{\prime} H\left[R_{1}\right]+\left(-a_{2}^{\prime}+a_{3}^{\prime} R_{1}\right) H\left[R_{1}-R^{\prime}\right]\right)\left|\theta_{B_{1}}^{P}-\theta_{A}^{P}\right| \\
+\left(a_{1}^{\prime} H\left[R_{2}\right]+\left(-a_{2}^{\prime}+a_{3}^{\prime} R_{2}\right) H\left[R_{2}-R^{\prime}\right]\right)\left|\theta_{B_{1}}^{P}-\theta_{C}^{P}\right| \\
+2 G \sqrt{\left(x_{B_{1}}^{P}-x_{B_{2}}^{P}\right)^{2}+\left(y_{B_{1}}^{P}-y_{B_{2}}^{P}\right)^{2}}
\end{gathered}
$$

where $R_{1}$ and $R_{2}$ are the radii of the first and second circles (Fig. 5), respectively. Also, $\theta_{B_{1}}^{P}, \theta_{A}^{P}$, and $\theta_{C}^{P}$ are the heading angles of the path at points $B_{1}$, $A$, and $C$ in the path-defined coordinate system (see Fig. 1 also), respectively. In addition, $\left(x_{B_{1}}^{P}, y_{B_{1}}^{P}\right)$ and $\left(x_{B_{2}}^{P}, y_{B_{2}}^{P}\right)$ are the position of points $B_{1}$ and $B_{2}$ in the path-defined coordinate system.

The optimally energy-efficient CLC path problem defined in section 2 can be written as follows:
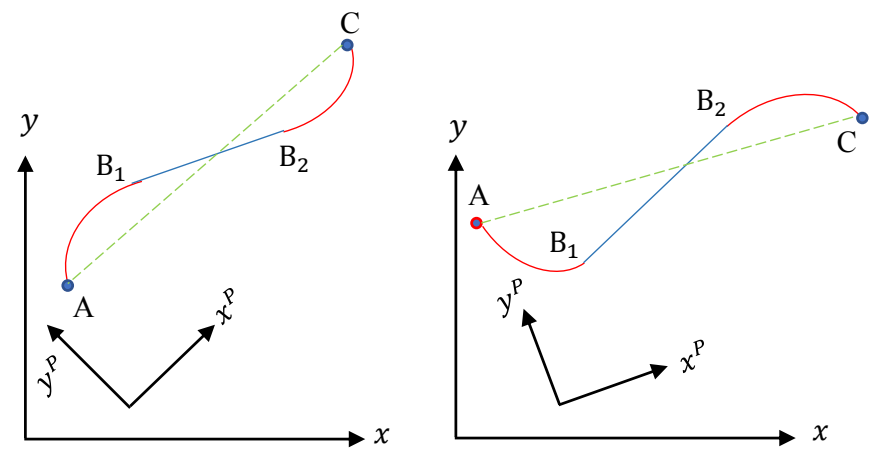

Fig. 5: CLC paths between a starting and end point in the global and path-defined coordinate system. The axes $x^{P}$ and $y^{P}$ indicate the path-defined coordinate system. Also, axes $x$ and $y$ indicate the global coordinate system. 


$$
\begin{array}{cl}
\underset{R_{1}, R_{2}, \theta_{B}^{P}}{\operatorname{minimize}} & E \\
\text { subject to } & h_{i}=0, i=1,2 \\
& 0<k \theta_{B_{1}}^{P} \leq \frac{\pi}{2} \\
& R_{j}>0, j=1,2
\end{array}
$$

To avoid searching for $\theta_{B_{1}}^{P}>2 \pi$ or $\theta_{B_{1}}^{P}<-2 \pi$ in the optimization process, the inequality constraints in (46) are written; it is clear when (50), written based on the simplifying assumption mentioned in section 2 , is held then $0<$ $k \theta_{B_{1}}^{P} \leq \frac{\pi}{2}$ is required for minimum energy. Also, $E$ is given by (45), and the equality constraints $\left(h_{i}\right)$ which define the CLC paths are as follows:

$$
\begin{gathered}
h_{1}=x_{B_{2}}^{P}-x_{B_{1}}^{P}-\cos \theta_{B_{1}}^{P} \sqrt{\left(x_{B_{2}}^{P}-x_{B_{1}}^{P}\right)^{2}+\left(y_{B_{2}}^{P}-y_{B_{1}}^{P}\right)^{2}} \\
h_{2}=y_{B_{2}}^{P}-y_{B_{1}}^{P}-\left(x_{B_{2}}^{P}-x_{B_{1}}^{P}\right) \tan \theta_{B_{1}}^{P}
\end{gathered}
$$

where

$$
\begin{aligned}
x_{B_{1}}^{P} & =x_{A}^{P}+k R_{1}\left(-\sin \theta_{A}^{P}+\sin \theta_{B_{1}}^{P}\right) \\
y_{B_{1}}^{P} & =y_{A}^{P}+k R_{1}\left(\cos \theta_{A}^{P}-\cos \theta_{B_{1}}^{P}\right) \\
x_{B_{2}}^{P} & =x_{C}^{P}+k R_{2}\left(\sin \theta_{C}^{P}-\sin \theta_{B_{1}}^{P}\right) \\
y_{B_{2}}^{P} & =y_{C}^{P}+k R_{2}\left(-\cos \theta_{C}^{P}+\cos \theta_{B_{1}}^{P}\right) \\
k & =-\frac{\sin \theta_{A}^{P}}{\left|\sin \theta_{A}^{P}\right|}, \theta_{A}^{P} \neq 0
\end{aligned}
$$

and

$$
\left\{\theta_{A}^{P}, \theta_{C}^{P} \in\left(0, \frac{\pi}{2}\right]\right\} \text { Or }\left\{\theta_{A}^{P}, \theta_{C}^{P} \in\left[-\frac{\pi}{2}, 0\right)\right\}
$$

which means $\theta_{A}^{P}$ and $\theta_{C}^{P}$ are both in the first or both in the fourth quadrant of path-defined coordinate system (see Fig. 5).

\section{Simulation and Experimental Results of CLC and PLP Paths}

This section presents simulation and experimental results for optimizing CLC paths (i.e. Problem 1 in section 2). Numerical results of the optimization set up in the preceding section are presented. CLC paths are performed experimentally on hard ground with a Husky rover, shown in Figure 6, in addition to PLP paths with the same start/end poses for comparison. Furthermore, some of the performed experimental tests are discussed in additional detail, as are the results of experiments exploring power consumption as a function of turning radius.

Relevant rover parameters are given in Table 2. Note that a weight (bin filled with sand) is attached to the rover such that the resulting center of mass is at the rover's geometric center; this increases the rover's total mass. 
Table 1: Husky Rover Test Plan on Hard Ground

\begin{tabular}{|c|c|c|c|}
\multicolumn{4}{|c|}{ Table 1: Husky Rover Test Plan on Hard Ground } \\
\hline \multirow{2}{*}{ Terrain } & Maneuver & $\left(\theta_{A}^{P}, \theta_{C}^{P}\right)$ & Commanded $\|\overrightarrow{A C}\|$ for Husky \\
\hline \hline \multirow{2}{*}{ Concrete } & \multirow{2}{*}{ CLC } & $\left(45^{\circ}, 45^{\circ}\right)$ & $10 \mathrm{~m}$ \\
\cline { 3 - 5 } & & $\left(60^{\circ}, 30^{\circ}\right)$ & $8 \mathrm{~m}$ \\
\hline \multirow{2}{*}{ Concrete } & \multirow{2}{*}{ PLP } & $\left(45^{\circ}, 45^{\circ}\right)$ & Measured Dis. for CLC $\left(45^{\circ}, 45^{\circ}\right)$ \\
\cline { 3 - 4 } & & $\left(60^{\circ}, 30^{\circ}\right)$ & Measured Dis. for CLC $\left(60^{\circ}, 30^{\circ}\right)$ \\
\hline
\end{tabular}

\subsection{Test Plan}

This subsection provides the plan for both the numerical and experimental tests. Because of the skid-steer rovers' ability to do point turns the simplest way to traverse a distance between two points is by doing a PLP. However, we will show that CLC paths can consume less energy than PLP paths. Accordingly, different scenarios for CLC and PLP paths are considered for the Husky, in simulation and in experiments on hard ground (concrete).

The test plan is shown in Table 1. Two scenarios are studied on hard ground, one with equal start and end angle, relative to the path-defined coordinate system (whose $\mathrm{x}$-axis is aligned with the line connecting points $\mathrm{A}$ and $\mathrm{C})$, and another with different start and end angles.

\subsection{Numerical Solutions of the Optimization Problem}

This subsection addresses the numerical optimization of CLC paths by utilizing the optimization problem formulated in subsection 3.2.

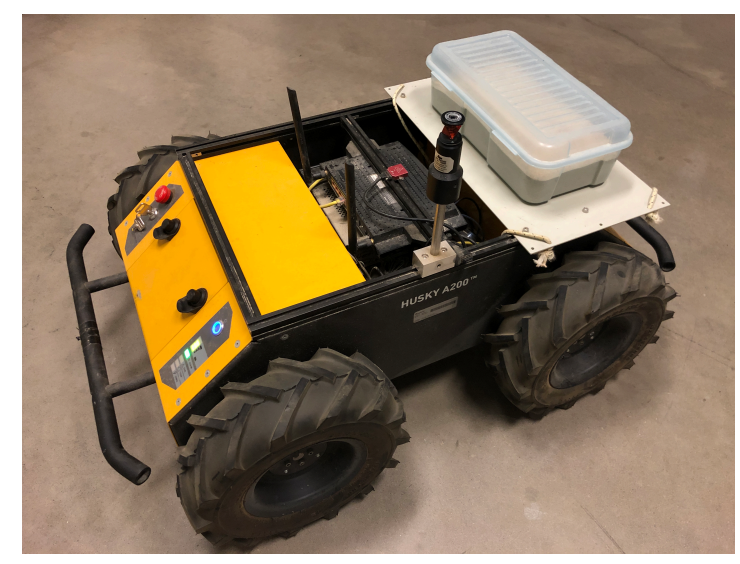

Fig. 6: Husky UGV Rover 
Table 2: Husky Rover Parameters on Hard Ground (HG) used in the Numerical Computations

\begin{tabular}{|c||c|c|c|c|c|c|c|c|c|}
\hline Parameter & $\mu$ & $G$ & $\left(\left|V_{l}\right|+\left|V_{r}\right|\right)$ & $L$ & $W$ & $\mathrm{~m}$ & $\mathrm{~g}$ & $p$ & $B_{s}$ \\
\hline Value & 0.74 & 30.85 & 0.6 & 0.52 & 0.55 & 63 & 9.81 & $\mathrm{mg} / 4$ & 1.29 \\
\hline Unit & - & $\mathrm{Ws} / \mathrm{m}$ & $\mathrm{m} / \mathrm{s}$ & $\mathrm{m}$ & $\mathrm{m}$ & $\mathrm{kg}$ & $\mathrm{m} / \mathrm{s}^{2}$ & $\mathrm{~N}$ & $\mathrm{~m}$ \\
\hline
\end{tabular}

Table 3: Numerically Computed Energy-Efficient CLC Paths for Husky Rover on Hard Ground

\begin{tabular}{ccccc}
\hline \hline Path Type & $\left(\theta_{\mathrm{A}}^{o}, \theta_{\mathrm{C}}^{o}\right)$ & Distance $(\mathrm{m})$ & $\left(R_{1}(\mathrm{~m}), R_{2}(\mathrm{~m}), \theta_{\mathrm{B}}(\mathrm{rad})\right)$ & Energy $(\mathrm{J})$ \\
\hline CLC & $(45,45)$ & 10 & $(0.645,0.645,-0.0417)$ & 965.83 \\
PLP & $(45,45)$ & 10 & $(0,0,0)$ & 1004.38 \\
\hline CLC & $(60,30)$ & 8 & $(0.645,0.645,-0.0577)$ & 851.21 \\
PLP & $(60,30)$ & 8 & $(0,0,0)$ & 880.98 \\
\hline
\end{tabular}

Parameters used to compute energy for the various scenarios listed in the test plan (Table 1) are provided in Table 2 for the Husky on hard ground. The parameters $\mu$ (friction coefficient), $G$ (internal/rolling resistance coefficient), and $B_{s}$ (slip track) are obtained experimentally, and are dependent on roverterrain combination. $B_{s}$ is calculated from the experimental data by using an Extended Kalman Filter, according to the procedure outlined by Pentzer et al. [28]. $G$ is obtained from straight-line maneuvers given $V_{l}, V_{r}$ and the measured power, using (20). Then, CLC maneuvers are utilized to calculate $\mu$ by using (20) and the calculated $G$.

To solve the constrained optimization problem presented by (46) in 3.2, the minimize function from the scipy.optimize package in python is used. In the minimize function, Sequential Least Squares Programming (SLSQP) algorithm is chosen. The optimization results are presented in the rows marked 'CLC' in Table 3 for the Husky on hard ground. Comparing the optimal turning radii returned by the optimization, in Table 3 to the $B_{s}$ input parameter in Table 2, it is clear that, for the tested start/end conditions, $R_{1}^{*}=R_{2}^{*}=\frac{B_{s}}{2}=$ $R^{\prime}$. The fact that $R^{\prime}$ is the optimal turning radius predicted by these numerical analyses highlights its importance.

Table 3 also provides the computed energy for PLP paths that achieve the same displacement from start to end pose. In both cases, the optimal CLC path is predicted to require less energy than the corresponding PLP path.

\subsection{Experimental results on hard ground}

This subsection studies the scenarios presented in Table 1 experimentally. To command a CLC path experimentally, the total desired Euclidean distance 
(between points $\mathrm{A}$ and $\mathrm{C}$ ), the start and end angles, and the commanded radii $R_{1}$ and $R_{2}$ are used to find the required angle subtended by each circular arc (at the center of the corresponding circle). Each circular arc is commanded until the rover's Inertial Measurement Unit (a VectorNav VN-100R) shows the required turn angle has been achieved. The straight line segments are commanded via dead-reckoning that accounts for an empirically estimated slip ratio. Because in practice the specified distances are not exactly achieved, the actually displaced Euclidean distance from start to end pose (measured using a laser total station shown in Fig. 7) in the CLC path is used to prescribe the distance for the PLP path. The final displacements from start to end poses for CLC and PLP paths are confirmed to be within $0.5 \%$ of one another, to ensure a fair comparison.

Rover power consumption (and energy consumption over each path) is measured with a TI INA226 bidirectional current and power sensor on the Husky.

The following definitions are given to distinguish between the commanded and measured turning radii for the Husky UGV:

Definition $2 R_{H_{c}}^{\prime}$ is the commanded turning radius for the Husky UGV that results in the inner wheels receiving a commanded velocity of $0 . R_{H_{c}}^{\prime}=0.55 \mathrm{~m}$.

Definition $3 R_{H}^{\prime}$ is the actual turning radius that the Husky follows when $R_{H_{c}}^{\prime}$ is commanded to it.

Definition $4 R_{e}$ is the actual turning radius that a rover follows during any generic circular arc, as measured experimentally. As will be explained in this subsection, the commanded $R_{1}$ and $R_{2}$ are equal for all the experimental tests. Hence, the measured $R_{1}\left(R_{1}^{\mathrm{m}}\right)$ and measured $R_{2}\left(R_{2}^{\mathrm{m}}\right)$ will be equal. So, $R_{e}=R_{1}^{\mathrm{m}}=R_{2}^{\mathrm{m}}$.

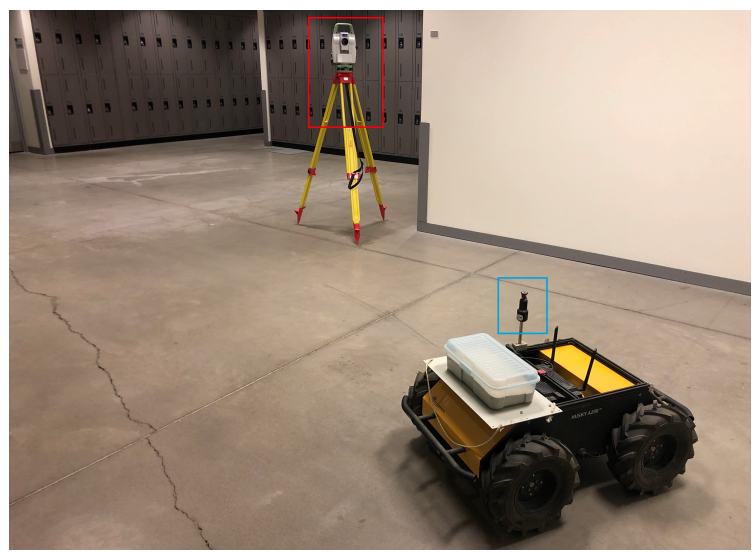

Fig. 7: The laser total station (red box) and the prism on the rover (blue box) used for tracking rover motion. 
It is worth noting that the $R_{H_{c}}^{\prime}$ of $0.55 \mathrm{~m}$ accounts for the fact that the rover will generally experience lateral slip. Clearpath Robotics have programmed a slip track, $B_{s}$, of $1.1 \mathrm{~m}$ into the Husky's controls for converting paired $v$ and $\omega_{z}$ commands into $V_{r}$ and $V_{l}$ control inputs according to (13) and (14). For hypothetical operation without lateral slip, the slip track would have been exactly equal to the rover width, but this programmed slip track is about twice the rover width; this highlights the important distinction between $R_{c}=W / 2$ and $R^{\prime}=B_{s} / 2$. Clearpath's programmed slip track is presumably based on some average slip track measured from tests on reference terrain(s). In practice, the actual slip track differs from this value.

In the case where the Husky is commanded with $R_{H_{c}}^{\prime}, R_{e}=R_{H}^{\prime}$. However, other turning radii can also be, and are, explored experimentally. For each test, the actual turning radius driven was derived from laser total station and IMU measurements. Because the offset between the total station prism (attached to the rover) and the rover center is known and constant, the rover center location can be computed by a simple transformation knowing the prism location and rover heading angle. A circle is fit to the rover center data points to find an actual turning radius. Note that the associated timestamps can further be used to compute the rover's forward velocity, angular velocity, and the velocity of its left and right sides. On concrete $R_{H}^{\prime}=0.645 \mathrm{~m}$.

In subsection 4.2 , it is shown that the minimum energy is always numerically predicted at $R_{1}=R_{2}=R^{\prime}$ ( $R_{\mathrm{H}}^{\prime}$ for Husky). Therefore, several CLC maneuvers are commanded around $R_{H_{c}}^{\prime}$ for the Husky UGV. Specifically, tests are performed with the following commanded turning radii: $0.5 R_{H_{c}}^{\prime}, R_{H_{c}}^{\prime}, 1.5 R_{H_{c}}^{\prime}$, $2 R_{H_{c}}^{\prime}$. Each of these tests is compared to its own respective PLP test (i.e. commanded turning radius of 0 ) for the fairest possible comparison, as described earlier. In these tests, the difference in total distance for all the CLC/PLP pairs was always less than $0.5 \%$, with the PLP path being slightly shorter.

The energy consumption of each CLC path $\left(E_{\mathrm{CLC}}\right)$ is compared to the energy consumption of the corresponding PLP path $\left(E_{\mathrm{PLP}}\right)$, quantified as a percentage difference given by the following equation:

$$
\frac{E_{\mathrm{CLC}}-E_{\mathrm{PLP}}}{E_{\mathrm{PLP}}} \times 100 \%
$$

In cases where the above metric is negative, the energy consumed by the CLC path is lower than the corresponding PLP path. Figure 8 and Figure 9 show the experimental results for this metric for the hard ground experiments described in the test plan, Table 1 . For each example path, 3 runs were conducted at each commanded turning radius, for a total of 12 CLC paths; 12 corresponding PLP paths were also run. For both example paths studied, the lowest energy consumption on average is observed for CLC paths with turning radii $R_{H}^{\prime}$, exactly as predicted by the numerical analysis (see Table 3 ).

Figure 10 compares the power consumption profiles for a PLP path versus its corresponding CLC path with $R=R_{H}^{\prime}$. The example shown is Run 1 from Fig. 8 (at $R_{e} / R_{H}^{\prime}=1$ ), i.e. with start and end angles of $45^{\circ}$ and a displacement of $10 \mathrm{~m}$. The total time required for the CLC maneuver is shorter, because 


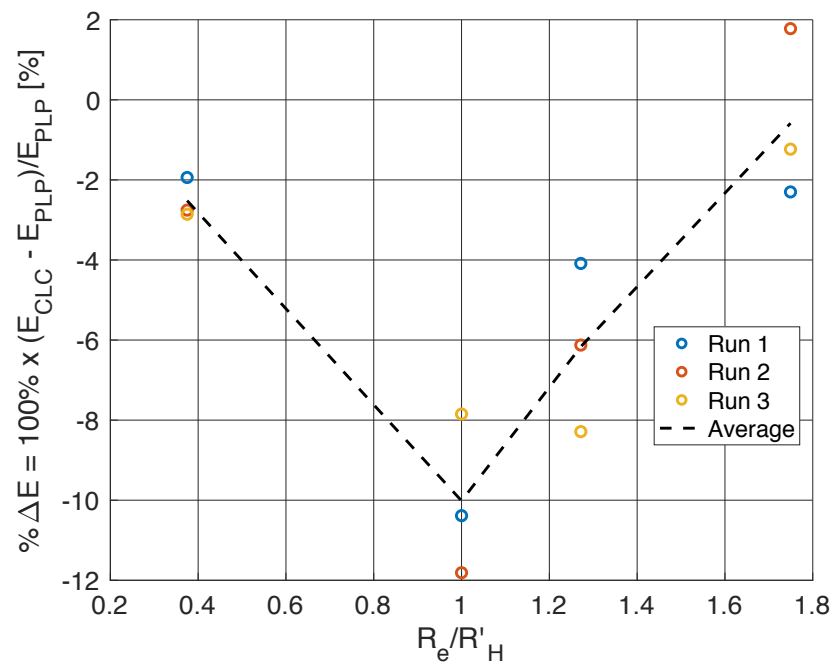

Fig. 8: Comparison of experimentally measured energy consumption for CLC paths vs. PLP paths for the Husky rover on hard ground. Start and end angle $45^{\circ}$; distance $10 \mathrm{~m}$. Lowest energy is observed for CLC paths with turning radius $R_{H}^{\prime}$.

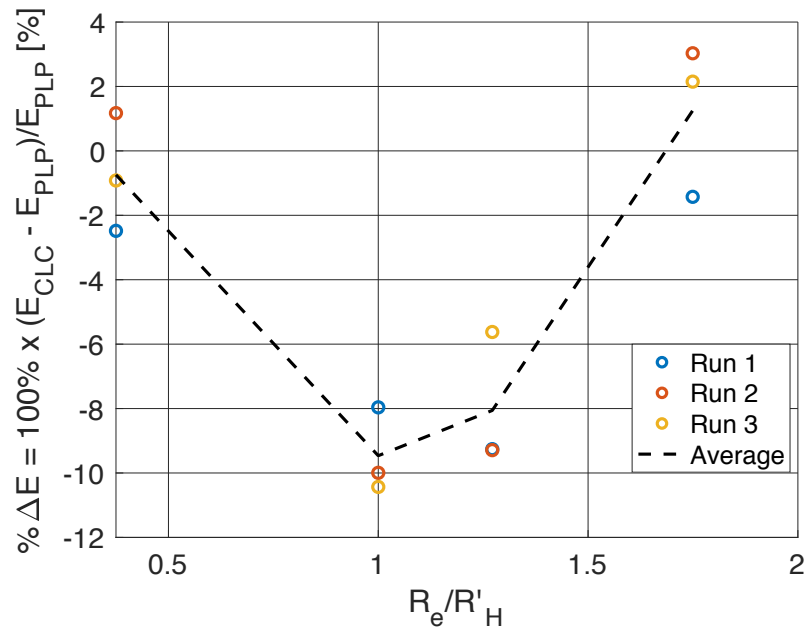

Fig. 9: Comparison of experimentally measured energy consumption for CLC paths vs. PLP paths for the Husky rover on hard ground. Start angle $60^{\circ}$; end angle $30^{\circ}$; distance $8 \mathrm{~m}$. Lowest energy is observed for CLC paths with turning radius $R_{H}^{\prime}$. 


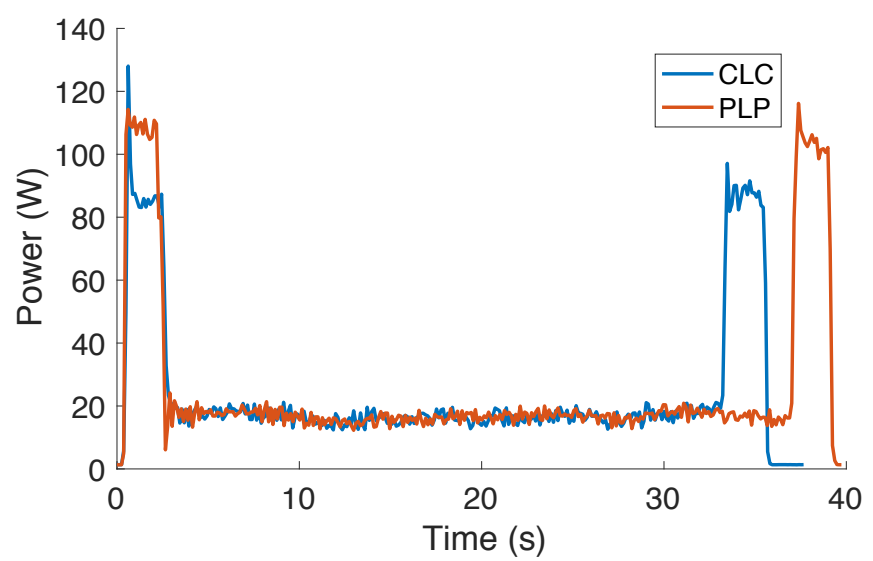

Fig. 10: Comparison of experimentally measured power consumption vs. time for a CLC path (with $R=R_{H}^{\prime}$ ) and PLP path for the Husky rover on hard ground.

the straight line segment is shorter due to the $R^{\prime}$ turn contributing to forward progress (as opposed to a point turn). The average power during the $R^{\prime}$ turns is also lower than point turns on this terrain. The time required to complete each arc is only slightly longer than it takes to complete the point turn. Taken together, the total energy is approximately $10 \%$ lower for the CLC path with $R=R^{\prime}$ (which we can call the $R^{\prime}$-CLC path) compared to the PLP path, as can be seen again in Fig. 8 .

\subsection{Experimental Power for Husky UGV}

This subsection presents power data measured experimentally for a skid-steer rover on hard ground, and discusses nuanced differences observed between this data and points discussed in prior literature.

A Husky UGV was commanded to execute 90 degree turns at various turning radii, subject to a constant forward velocity of $0.3 \mathrm{~m} / \mathrm{s}$, on hard ground (concrete). Power was measured for both the left and right motors using a Texas Instruments INA226 bidirectional current and power sensor, and the total power was averaged over the duration of the 90 degree turn. The turns start at the commanded $R_{H_{c}}^{\prime}$ and explore left and right power consumption as the turning radius is increased towards infinity.

Experimental data collected on hard ground (Figure 11) shows approximate correspondence with observations made in prior literature. It can also be seen that inner wheel power consumption becomes negative after $R^{\prime}$, as observed previously above $R_{c}$ by Dogru and Marques [22,23]. However, once the turning radius increases past a certain point (18 $\mathrm{m}$ on this particular terrain), 


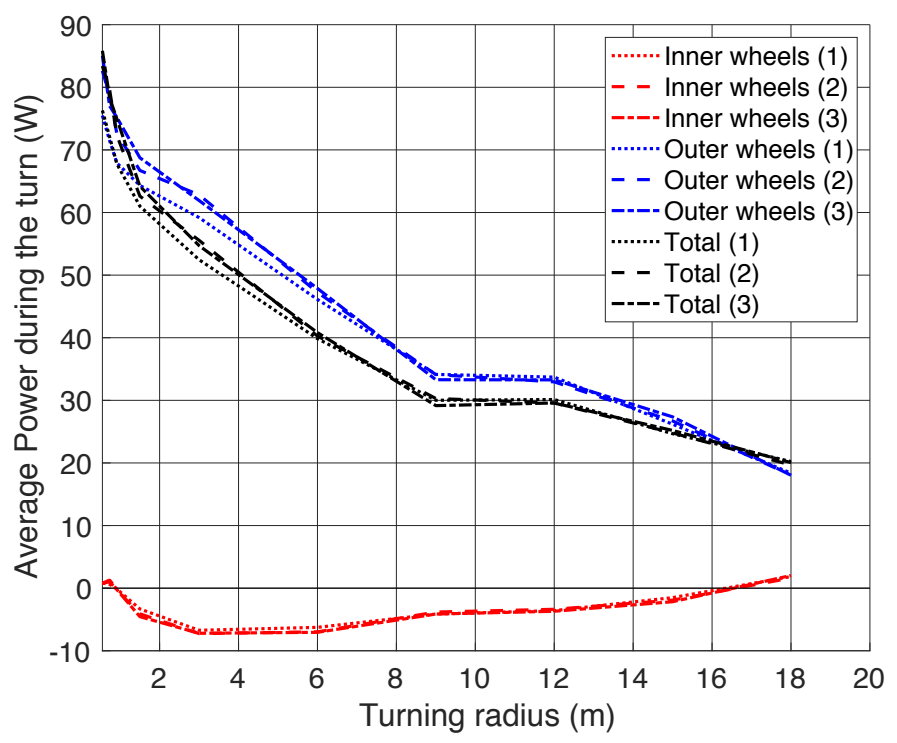

Fig. 11: Husky UGV power consumption as a function of commanded turning radii bigger than $R^{\prime}$. The tests are performed on hard ground consisting of concrete.

inner wheel power consumption becomes positive again. This is consistent with a reasonable expectation that power consumption changes gradually at large turning radii, rather than jumping suddenly to be negative as soon as left and right wheel velocities become unequal (i.e. the power does not remain negative for all of $R^{\prime}<R<\infty$, but rather for $R^{\prime}<R<R_{T}$, where $R_{T} \ll \infty$ is a threshold radius).

\subsection{Interpretation and generalization of optimization results}

The simulated and experimental results on hard ground both show optimal energy consumption with CLC paths occurs with turning radii of $R^{\prime}$. It is instructive to now re-examine theory presented earlier in light of this result.

For $0 \leq R \leq R^{\prime}$, Theorem (1) shows that energy consumed by a turn is a constant times $|\Delta \theta|$. In other words, the energy required to change heading is the same regardless of whether a point turn or $R^{\prime}$ turn is employed. However, when constructing a CLC path this choice affects the length of the straight line, and thus $E_{B_{1} B_{2}}$. In general, $R^{\prime}$-CLC paths require more $|\Delta \theta|$ than PLP paths, but as the distance between the start and end points is increased, the 
difference approaches zero. On the other hand, because the $R^{\prime}$ turns also make forward progress, the line in a CLC path will be shorter than a PLP path. This is exactly what is seen in the simulations and experiments explored in the preceding subsections.

The above argument does not necessarily hold for small distances between $\mathrm{A}$ and $\mathrm{C}$, and future work will explore the threshold at which $R^{\prime}$ curves stop being the optimal choice.

\section{Conclusion}

This research identifies $R^{\prime}$, equal to half a skid-steer rover's slip track and also the radius at which the inner wheels are not commanded to turn, as a crucial concept in understanding the energy-efficiency of skid-steer rovers. When considered with respect to a popular power model for skid-steer rovers, $R^{\prime}$ corresponds to an important transition for energy consumption: below $R^{\prime}$ energy consumption is not a function of turning radius while above $R^{\prime}$ it is.

This research searches, both in numerical simulation and in experiments on hard ground, for the most energy-efficient path from among Circular arc Line - Circular arc (CLC) paths, a generalization of Point turn - Line - Point turn (PLP) paths (PLP paths are the simplest path to execute for a skidsteer rover traversing between general start and end poses). Because energy consumption is not a function of $R$ below $R^{\prime}$, an $R^{\prime}$ circular arc requires the same energy as a point turn through the same angle, but achieves forward as well that shortens the straight line portion of the path. Therefore, $R^{\prime}$-CLC paths (or perhaps just straight lines) should be used as "optimistic" estimates of cost to a goal, rather than PLP paths.

From the evaluations and investigations reported here, the following findings are concluded:

- Numerically optimizing CLC paths for energy-efficiency shows that, for the existing power model and all cases examined, $R=R^{\prime}$ gives the minimum energy consumption.

- Results obtained from experiments on hard ground (concrete) for CLC paths also show minimum energy consumption with $R=R^{\prime}$, thus agreeing with the numerical predictions regarding the importance of this special turning radius.

- Experimental results indicate that in the example scenarios studied, with the end goal several rover lengths away from the start point, there is always a CLC path that can be found that is more efficient than its corresponding PLP path.

- When examining power consumption of inner and outer wheels during a skid-steer turn, inner wheel power does not remain negative for all of $R^{\prime}<R<\infty$, but rather for $R^{\prime}<R<R_{T}$, where $R_{T} \ll \infty$ is a threshold radius.

The following areas are identified as useful directions for future work: 
- Devising optimally energy-efficient non-predefined paths for skid-steer rovers.

- Adapting global path planners to include $R^{\prime}$ CLC paths as local planning methods. This could include adaptively extending nodes away from obstacles to give room for wider turning radii when required to maintain collision-free paths.

- Extending the analysis to soft soils, including modifying the power model to account for differences in the rover-terrain interactions.

\section{Acknowledgment}

The authors acknowledge financial support from the Natural Sciences and Engineering Research Council of Canada (NSERC) as well as funding and technical collaboration from Mission Control Space Services Inc., and thank the Canadian Space Agency (CSA) for facilitating access to their Mars analogue terrain.

\section{References}

1. Kassel, C.: Lunokhod-1 Soviet lunar surface vehicle. RAND CORP SANTA MONICA CA, Tech. Report, Vol. 802 (1971).

2. Reid, E., Iles, P., Muise, J., Cristello, N., Jones, B., Faragalli, M., Visscher, P., Boucher, D., Simard-Bilodeau, V., Apostolopoulos, D., and Rocco, P.: The Artemis Jr. rover: Mobility platform for lunar ISRU mission simulation. Advances in Space Research, vol. 55, no. 10, 2472-2483 (2015).

3. Shamah, B.: Experimental Comparison of Slid Steering vs. Explicit Steering for a Wheeled Mobile Robot. M. S. Thesis in Robotics, Carnegie Mellon University, 1998.

4. LaValle, S.M.: Planning algorithms. Cambridge university press (2006).

5. Raja, R., Dutta, A., and Venkatesh, K. S.: New potential field method for rough terrain path planning using genetic algorithm for a 6 -wheel rover. Robotics and Autonomous Systems, vol. 72, 295-306 (2015).

6. Kewlani, G., Ishigami, G., and Iagnemma, K.: Stochastic mobility-based path planning in uncertain environments. In Intelligent Robots and Systems Conference (IROS), pp. 1183-1189 (2009).

7. LaValle, S.M.: Rapidly-exploring random trees: A new tool for path planning. Report No. TR 98-11, Computer Science Department, Iowa State University.

8. LaValle, S.M., and Kuffner, J.J.: Randomized kinodynamic planning. International Journal of Robotics Research, vol. 20, no. 5, 378-400 (2001).

9. Karaman, S., and Frazzoli, E.: Incremental sampling-based algorithms for optimal motion planning. Robotics Science and Systems, vol. 104 (2010).

10. Palmieri, L., Koenig, S., and Arras, K.O.: RRT-based nonholonomic motion planning using any-angle path biasing. In Robotics and Automation (ICRA) Conference, pp. 2775-2781 (2016).

11. Stentz, A.: Optimal and Efficient Path Planning for Partially-Known Environments. Proceedings of the International Conference on Robotics and Automation, pp. 33103317 (1994).

12. Dunlap, D.D., Caldwell, C.V., Collins, E.G., and Chuy, O.: Motion planning for mobile robots via sampling-based model predictive optimization. In Recent Advances in Mobile Robotics. InTech, (2011).

13. Reese, B.M.: A graph based approach to nonlinear model predictive control with application to combustion control and flow control. Florida A and M University Tallahassee United States, Tech. Report, W911NF-13-1-0122 (2015). 
14. Gupta, N., Ordonez, C., and Collins, E.G.: Dynamically feasible, energy efficient motion planning for skid-steered vehicles. Autonomous Robots, vol. 41, no. 2, 453-471 (2017).

15. Pentzer, J., Reichard, K., and Brennan, S.: Energy-based path planning for skid-steer vehicles operating in areas with mixed surface types. In American Control Conference (ACC), 2110-2115 (2016).

16. Tokekar, P., Karnad, N., and Isler, V.: Energy-optimal trajectory planning for car-like robots. Autonomous Robots, vol. 37, no. 3, 279-300 (2014).

17. Dubins, L.E.: On curves of minimal length with a constraint on average curvature, and with prescribed initial and terminal positions and tangents. American Journal of mathematics, vol. 79, no. 3, 497-516 (1957).

18. Reeds, J. and Shepp, L.: Optimal paths for a car that goes both forwards and backwards. Pacific journal of mathematics, vol. 145, no. 2, 367-393 (1990).

19. Chitsaz, H., LaValle, S.M., Balkcom, D.J., and Mason, M.T.: Minimum wheel-rotation paths for differential-drive mobile robots. In International Conference on Robotics and Automation (ICRA), pp. 1616-1623 (2006).

20. Balkcom, D.J., and Mason, M.T.: Time optimal trajectories for bounded velocity differential drive vehicles. The International Journal of Robotics Research, vol. 21, no. 3, 199-217 (1979).

21. Effati, M., and Skonieczny, K.: Circular ARC-Based Optimal Path Planning for SkidSteer Rovers. Canadian Conference on Electrical and Computer Engineering (CCECE), pp. 1-4 (2018).

22. Dogru, S. and Marques, L.: A Physics-Based Power Model for Skid-Steered Wheeled Mobile Robots. IEEE TRANSACTIONS ON ROBOTICS, vol. 34, no. 2, 421-433 (2018).

23. Dogru, S., and Marques, L.: Power Characterization of a Skid-Steered Mobile Field Robot with an Application to Headland Turn Optimization. Journal of Intelligent and Robotic Systems, vol. 93, no. 3, 601-615 (2019).

24. Morales, J., Martnez, J.L., Mandow, A., Pequeo-Boter, A., and Garca-Cerezo, A.: Simplified power consumption modeling and identification for wheeled skid-steer robotic vehicles on hard horizontal ground. 2010 IEEE/RSJ International Conference on Intelligent Robots and Systems, Taipei, pp. 4769-4774 (2010).

25. Mandow, A., Martinez, J.L., Morales, J., Blanco, J.L., Garcia-Cerezo, A., and Gonzalez, J.: Experimental kinematics for wheeled skid-steer mobile robots. 2007 IEEE/RSJ International Conference on Intelligent Robots and Systems, San Diego, CA, pp. 1222-1227 (2007).

26. Martnez, J.L., Mandow, A., Morales, J., Pedraza, S., and Garca-Cerezo, A.: Approximating kinematics for tracked mobile robots. The International Journal of Robotics Research, vol. 24, no. 10, 867-878 (2005).

27. Morales, J., Martinez, J.L., Mandow, A., Garcia-Cerezo, A.J., and Pedraza, S.: Power Consumption Modeling of Skid-Steer Tracked Mobile Robots on Rigid Terrain. IEEE Transactions on Robotics, vol. 25, no. 5, 1098-1108 (2009).

28. Pentzer, J., Brennan, S., and Reichard, K.: Model-based Prediction of Skid-steer Robot Kinematics Using Online Estimation of Track Instantaneous Centers of Rotation. Journal of Field Robotics, vol. 31, no. 3, 455-476 (2014). 